\title{
The Local Ly $\alpha$ Forest. I. Observations with the GHRS/G160M on the Hubble Space Telescope ${ }^{1}$
}

\author{
Steven V. Penton, John T. Stocke, and J. Michael Shull ${ }^{2}$ \\ Center for Astrophysics and Space Astronomy, Department of Astrophysical and Planetary \\ Sciences, University of Colorado, Boulder CO, 80309 \\ spenton@casa.colorado.edu, stocke@casa.colorado.edu, mshull@casa.colorado.edu
}

\begin{abstract}
We present the target selection, observations, and data reduction and analysis process for a program aimed at discovering numerous, weak (equivalent width $\leq 100 \mathrm{~m} \AA$ ) Ly $\alpha$ absorption lines in the local Universe $(0.003 \leq z \leq 0.069)$. The purpose of this program is to study the physical conditions of the local intergalactic medium, including absorber distributions in Doppler width and H I column density, redshift evolution of absorber number density, line-of-sight two-point correlation function, and the baryonic content and metallicity. By making use of large-angle, nearby galaxy redshift surveys, we will investigate the relationship (if any) between these Ly $\alpha$ absorbers and galaxies, superclusters and voids. In Paper I, we present high resolution $\left(\sim 19 \mathrm{~km} \mathrm{~s}^{-1}\right)$ spectroscopic observations of 15 very bright $(V \leq 14.5)$ AGN targets made with the Goddard High Resolution Spectrograph (GHRS) aboard the Hubble Space Telescope (HST). We find 81 definite $(\geq 4 \sigma)$ and 30 possible $(3-4 \sigma)$ Ly $\alpha$ absorption lines in these spectra, which probe a total pathlength of $116,000 \mathrm{~km} \mathrm{~s}^{-1}(\Delta z \sim 0.4)$ at very low redshift $(z \leq 0.069)$ and column density $\left(12.5 \leq \log N_{\mathrm{HI}} \leq 14.5\right)$. We found numerous metal lines arising in the Milky Way halo, including absorption from high velocity clouds in 10 of 15 sightlines and numerous absorptions intrinsic to the target AGN. Here, we describe the details of the target selection, HST observations, and spectral reduction and analysis. We present reduced spectra, absorption line lists, "pie diagrams" showing the known galaxy distributions in the direction of each target, and nearest galaxy tables for each absorber. In Papers II and III, we use the data presented here to determine the basic physical characteristics of the low- $z \operatorname{Ly} \alpha$ forest and to investigate the relationship of the absorbers to the local galaxy distribution.
\end{abstract}

Subject headings: intergalactic medium — quasars: absorption lines — ultraviolet: galaxies — galaxies: halos

\footnotetext{
${ }^{1}$ Based on observations with the NASA/ESA Hubble Space Telescope, obtained at the Space Telescope Science Institute, which is operated by the Association of Universities for Research in Astronomy, Inc. under NASA contract No. NAS5-26555.

${ }^{2}$ Also at JILA, University of Colorado and National Institute of Standards and Technology.
} 


\section{Introduction}

Since the discovery of the high-redshift Ly $\alpha$ forest over 25 years ago, these abundant absorption features in the spectra of QSOs have been used as evolutionary probes of the intergalactic medium (IGM), galactic halos, large-scale structure, and chemical evolution. These absorbers were originally divided into "metal-bearing" systems, which occur primarily at higher H I column densities, and Ly $\alpha$-only systems, which occur primarily at lower H I column densities (Sargent 1987). Recent observations of C IV lines in Ly $\alpha$ absorbers with column densities $N_{\mathrm{HI}} \geq 10^{14.5} \mathrm{~cm}^{-2}$ (Tytler et al. 1995; Cowie et al. 1995) have blurred the distinction between these two population of absorbers. However, the clustering properties of the high-redshift C IV systems are more like those of galaxies than the Ly $\alpha$ clouds (Cristiani et al. 1997). Certainly many of the higher column density absorbers, particularly the Lyman limit systems (Steidel 1995) and damped-Ly $\alpha$ systems (Wolfe 1995), appear to be associated with the gaseous halos of individual galaxies. Thus, the distinction between these two populations may still be an important one.

In the past few years, these discrete $\operatorname{Ly} \alpha$ lines have been interpreted theoretically by N-body hydrodynamical models (Cen et al. 1994; Hernquist et al. 1996; Zhang et al. 1997; Davé et al. 1999) as arising from baryon density fluctuations associated with gravitational instability during the epoch of structure formation. However, these models do not contain the detailed physics of star formation and supernova ejecta, which can move material from the disks of galaxies into the halo or beyond in a "galactic fountain" (Shapiro \& Benjamin 1993; Slavin, Shull, \& Begelman 1993). Therefore, these simulations cannot exclude the possibility that at least some Ly $\alpha$ absorbers are "recycled" gas from sites of active star formation in galaxies. Regardless, the lowest column density absorbers $\left(N_{\mathrm{HI}} \leq 10^{14.5} \mathrm{~cm}^{-2}\right)$ are the most likely systems to be unassociated with galaxies and primordial in their chemical abundances.

At high redshift, ground-based observations indicate rapid evolution in the distribution of Ly $\alpha$ absorption lines per unit redshift, $d \mathcal{N} / d z \propto(1+z)^{\gamma}$, where $\gamma \approx 2.5$ for $z \geq 1.5$. This evolution is consistent with a picture of these features as highly ionized "clouds" whose numbers and sizes were controlled by the evolution of the IGM pressure, the metagalactic ionizing radiation field, and galaxy formation. If the evolution in cloud numbers at $z>1.6$ continued into the present epoch, few Ly $\alpha$ clouds would remain by $z \sim 0$. However, one of the delightful spectroscopic surprises from the first year of the Hubble Space Telescope (HST) was the discovery of many Ly $\alpha$ absorption lines toward the quasar 3C 273 at $z_{\mathrm{em}}=0.158$ by both the Faint Object Spectrograph (FOS, Bahcall et al. 1991b) and the Goddard High Resolution Spectrograph (GHRS, Morris et al. 1991, 1993). More recent data from HST support the slowing of the evolution of Ly $\alpha$ systems at $z \leq 1.5$ (Weymann et al. 1998). This evolutionary shift is probably a result of the collapse and assembly of baryonic structures in the IGM (Davé et al. 1999) together with the decline in the intensity of the ionizing radiation field (Haardt \& Madau 1996; Shull et al. 1999b). Detailed results of the Ly $\alpha$ forest evolution in the redshift interval $0<z<1.5$ are described in the FOS Key Project papers: the three catalog papers (Bahcall et al. 1993, 1996; Jannuzi et al. 1998) and the evolutionary analysis (Weymann et al. 1998). 
Because the HST/FOS studies were primarily detections of strong Ly $\alpha$ lines, with equivalent widths (EWs) greater than $0.24 \AA$, they most likely probe only the higher column density population of absorbers. A great deal more information about the low- $z$ IGM can be gained from studies of the more plentiful weak absorbers, including the evolution of the lower column density population, which may be physically distinct. Realizing the importance of spectral resolution in detecting weak Ly $\alpha$ absorbers, the Colorado group has engaged in a long-term program with the HST/GHRS, using the G160M grating at $19 \mathrm{~km} \mathrm{~s}^{-1}$ resolution to study the very low-redshift $(z<0.07)$ Ly $\alpha$ forest. Earlier results from our study have appeared in various papers (Stocke et al. 1995; Shull, Stocke, \& Penton 1996; Shull et al. 1998) and reviews (Shull 1997; Shull, Penton, \& Stocke 1999a). While other groups have used the GHRS to probe these lower column density clouds (e.g., Tripp, Lu, \& Savage 1998a,b; Impey, Petry, \& Flint 1999), their observations used the lower-resolution GHRS/G140L grating, which failed to detect the weakest Ly $\alpha$ lines and did not resolve the stronger ones. The program described here detects lines as weak as $\mathrm{EW} \cong 13 \mathrm{~m} \AA\left(N_{\mathrm{HI}} \sim 10^{12.5} \mathrm{~cm}^{-2}\right)$, comparable to the detection limit reached with the Keck Observatory plus HIRES spectrograph at high redshift (Songaila, Cowie, \& Hu 1995). Because the HST/G160M resolves most of the Ly $\alpha$ absorption lines, it provides, for the first time, the range of observed Doppler parameters ( $b$-values) and the two-point correlation function of the low- $z$ Ly $\alpha$ forest at velocity separations $\leq 100 \mathrm{~km} \mathrm{~s}^{-1}$.

In order to attain the required resolution and sensitivity, we made two important compromises in the observing strategy. First, only the brightest UV targets could be used for the program; the rare Seyferts, QSOs, and BL Lacs with $\mathrm{V} \sim 14.5$ are our primary candidates. Second, because the HST/G160M grating yields only $\sim 35 \AA$ of useful coverage for Ly $\alpha$ forest lines, little pathlength is surveyed per target compared to either the FOS Key Project survey observations or the groundbased spectra at higher redshift. However, in many ways the $\sim 8,000 \mathrm{~km} \mathrm{~s}^{-1}$ pathlength surveyed by these spectra are the most important piece of the Ly $\alpha$ forest, being the nearest $\sim 100 \mathrm{Mpc}$ to us. The distances to Ly $\alpha$ absorbers discovered in the present survey allow an immediate comparison with galaxy locations derived from redshift surveys that sample approximately the same range in distances (e.g., the CfA redshift surveys, Huchra et al. 1992). One of the first discoveries of our program was that some Ly $\alpha$ absorbers are found in nearby galaxy "voids", providing the first detection of gas in those voids (Stocke et al. 1995; Shull, Stocke, \& Penton 1996). Additionally, the small distances (on the cosmic scale) to these absorbers allow deeper galaxy surveys in the optical and $21 \mathrm{~cm}$ emission line, from which the most sensitive limits have been set on the non-detection of galaxies nearby to Ly $\alpha$ clouds (Van Gorkom et al. 1993, 1996; Morris et al. 1993; Rauch, Weymann, \& Morris 1996; Shull et al. 1998; Impey, Petry, \& Flint 1999). Observations of the latter type led us to investigate strong Ly $\alpha$ systems toward PKS 2155-304 (Shull et al. 1998) with very low metal abundance, which may be among the first detected primordial H I clouds in the local Universe.

The first set of four papers discuss our full GHRS dataset of low column density, low- $z$ Ly $\alpha$ absorption systems. In Paper I (this article) we describe our HST/GHRS observations and catalog of Ly $\alpha$ absorbers. In Paper II (Penton, Shull, \& Stocke 2000a) we describe the primary scientific results from our program, including information on the physical parameters mentioned above and 
the nature of the low-redshift Ly $\alpha$ forest. In Paper III (Penton, Stocke, \& Shull 2000b) we discuss the relationship between the Ly $\alpha$ absorbers discovered in our GHRS program and the nearby galaxy distribution from available galaxy redshift surveys. In Paper IV (Penton, Stocke, \& Shull 2000c), we will combine these GHRS observations with results from 13 STIS sightlines to discuss the twopoint correlation function of the low- $z$ Ly $\alpha$ forest. These low- $z$ Ly $\alpha$ absorption lines, combined with information about the distribution of nearest galaxies, can probe large-scale baryonic structures (filaments) in the IGM, some of which may be remnants of physical conditions set up during the epoch of galaxy formation.

In this first paper, we describe data from the HST/GHRS of very bright AGNs, which we use to detect low column density $\left(\log N_{\mathrm{HI}}=12.5-14.5\right)$ Ly $\alpha$ absorption clouds at very low redshift

$(z<0.07)$. The GHRS with the G160M grating delivers spectral resolution $\mathrm{R} \sim 15,000$. Subsections of this paper describe the target selection and basic observational setup $(\S 2)$, calibration $(\S 2.2)$, spectral processing $(\S 2.3)$, and a thorough description of each sightline ( $\S 3$ ). This paper concludes with a tabulation of all quantities directly measured from the spectra.

\section{HST Observations, Calibration, and Spectral Processing}

\subsection{HST Observations}

To study the local Ly $\alpha$ forest, we used all available HST GHRS/G160M spectra that meet the following simple criteria: (1) Available: public domain GHRS/G160M spectra or our own HST Guest Observer observations, and (2) Appropriate: spectra blueward of the target's Ly $\alpha$ emission and in the wavelength range $1218-1300 \AA(0.002<z<0.069$ for Ly $\alpha)$. The majority of these observations were obtained from our HST Guest Observer projects 3584, 5715, and 6593. We selected our targets based upon two factors: brightness and location in the sky. To determine the brightest targets in the UV, we used the IUEAGN database (Penton, Shull, \& Edelson 2000d) to coadd all IUE spectra of AGN to determine the brightest UV targets with appropriate redshifts. We limited our selection to targets with $z_{\mathrm{em}}<0.18$, to avoid having Ly $\beta$ appear in our wavelength band of interest (1218-1300 $\AA$ ), but we required $z_{\mathrm{em}}>0.025$ to maximize pathlength beyond the region absorbed by the Milky Way interstellar H I. In project 3584 (which carried over into project $5715)$ we observed 4 objects behind well-studied galaxy voids in the foreground of the "Great Wall" (Stocke et al. 1995; Shull, Stocke, \& Penton 1996). In the hope of determining the relationship between galaxies and Ly $\alpha$ absorbers in the low- $z$ universe, we selected this region of the sky where the CfA galaxy redshift surveys were most complete (Huchra et al. 1992; Giovanelli \& Haynes 1984). Owing to the lack of sufficiently bright targets in the direction of the first CfA slice, in project 6593 we observed 7 additional targets in other directions. Our sample is rounded out by 5 archival spectra and a few additional exposures on targets that we had already observed. One of our archival targets is at higher $z$ than the above limit $(z=0.297 ; \mathrm{H} 1821+632)$ but is also included. In this sightline we take special care to differentiate Ly $\alpha$ lines from other lines, such as Ly $\beta$ for 
$z>0.19$ absorbers. Recent FUSE observations of several of these sightlines (Shull et al. 2000) detect Ly $\beta$ corresponding to the stronger Ly $\alpha$ absorbers, with EWs $>200 \mathrm{~m} \AA$. Our survey of low- $z$ Ly $\alpha$ clouds continues with HST/STIS observations of an additional 13 targets, which will be reported elsewhere.

Table 1 summarizes the HST observations and observational parameters of the forty-one GHRS/G160M exposures towards the 15 targets included in our dataset. The first column in Table 1 identifies the observed target, while the second column lists the HST exposure or dataset identifier. Additional columns provide: (3) the exposure calendar date, (4) whether the COSTAR (Corrective Optics Space Telescope Axial Replacement) optics were in place at the time of the exposure, (5) the exposure time (in seconds), (6 \& 7) the wavelength extent of the spectrum, (8) the HST/GHRS wavelength calibration (SPYBAL) exposure used in the calibration of the spectrum, and (9) the HST proposal identification number (PID) and Principal Investigator (PI) of the observation.

Table 2 summarizes the J2000 positions (columns 2-5) and redshifts ( $z$; column 6) of our HST targets. All redshifts are the optical, narrow-line, emission redshifts as reported from the NASA/IPAC Extragalactic Database (NED). ${ }^{3}$ Also included in Table 2, but discussed in detail later, are, by column: (7) the Galactic H I velocity centroid relative to the Local Standard of Rest (LSR) along this sightline as determined from the Leiden/Dwingeloo or Parkes multibeam survey, (8) the adjustment required to convert the observed wavelength scale to the LSR, where $\Delta V_{\mathrm{lsr}}=V_{\mathrm{lsr}}-V_{\mathrm{obs}}$, as determined from the location of the S II Galactic absorption lines (1250, 1253, and $1259 \AA$, see $\S 2.3 .3$ ) (9) the order $(\mathbf{O})$ of the polynomial used to normalize the continuum of each target, (10) the dominant H I $21 \mathrm{~cm}$ velocity of the Galactic High Velocity Clouds (HVCs) along each sightline as reported by Wakker \& van Woerden (1991), and (11) the mean signal-tonoise ratio $(\mathrm{S} / \mathrm{N})$ per resolution element $(\mathrm{RE})$ of each spectrum. The Galactic H I HVC velocities are taken from Wakker \& van Woerden (1991), and the Galactic H I LSR velocity measurements are taken from the Leiden/Dwingeloo survey (Hartmann \& Burton 1997). Note that Fairall 9, Mrk 335 and PKS 2155-304 are located near the Magellanic Stream (Gibson et al. 2000b), while Mrk 279, Mrk 290, Mrk 501, Mrk 817, and H 1821+643, are close to the HVC complex C (Gibson et al. 2000a). The sightline to Mrk 421 lies between the Galactic HVC MI and MII clouds, and the Mrk 509 sightline passes near the Galactic center negative velocity HVC feature. These objects are therefore more likely to contain Galactic absorption features at velocities other than the LSR.

\footnotetext{
${ }^{3}$ The NASA/IPAC Extragalactic Database (NED) is operated by the Jet Propulsion Laboratory, California Institute of Technology, under contract with NASA.
} 
Table 1: HST GHRS/G160M Observations

\begin{tabular}{|c|c|c|c|c|c|c|c|c|}
\hline Target & Dataset & $\overline{\text { Date }}$ & $\begin{array}{c}\text { COSTAR } \\
\text { Deployed ? }\end{array}$ & $\begin{array}{c}\mathrm{ET} \\
(\mathrm{sec})\end{array}$ & $\begin{array}{c}\lambda_{\text {start }} \\
(\AA)\end{array}$ & $\begin{array}{c}\lambda_{\text {end }} \\
(\AA) \\
\end{array}$ & Spybal & $\overline{\mathrm{PID} / \mathrm{PI}^{\mathrm{a}}}$ \\
\hline$(1)$ & $(2)$ & $(3)$ & (4) & (5) & (6) & $(7)$ & $(8)$ & (9) \\
\hline $3 \mathrm{C} 273$ & z0gu010mn & $02 / 23 / 91$ & No & 1824.8 & 1265.430 & 1301.460 & z0gu010nm & 1140/WEYMANN \\
\hline $3 \mathrm{C} 273$ & z0gu010om & $02 / 23 / 91$ & No & 2737.1 & 1234.680 & 1270.790 & z0gu010pm & 1140/WEYMANN \\
\hline $3 \mathrm{C} 273$ & z1760105t & $11 / 27 / 94$ & Yes & 1115.1 & 1214.110 & 1250.270 & z1760103t & 3951/WEYMANN \\
\hline $3 \mathrm{C} 273$ & z1760107t & $11 / 27 / 94$ & Yes & 1115.1 & 1214.130 & 1250.290 & z1760106t & 3951/WEYMANN \\
\hline $3 \mathrm{C} 273$ & z1760108t & $11 / 27 / 94$ & Yes & 1115.1 & 1214.130 & 1250.290 & z1760106t & 3951/WEYMANN \\
\hline AKN120 & z3e70604t & $12 / 26 / 96$ & Yes & 5702.4 & 1222.542 & 1258.674 & z3e70603t & 6593/STOCKE \\
\hline AKN120 & z3e70606t & $12 / 26 / 96$ & Yes & 5702.4 & 1222.587 & 1258.707 & z3e70605t & 6593/STOCKE \\
\hline AKN120 & z3e70608m & $12 / 27 / 96$ & Yes & 5702.4 & 1222.609 & 1258.726 & z3e70607t & 6593/STOCKE \\
\hline AKN120 & z3e7060at & $12 / 27 / 96$ & Yes & 2433.0 & 1222.638 & 1258.751 & z3e70609t & 6593/STOCKE \\
\hline ESO141-G55 & z3e70204t & $08 / 15 / 96$ & Yes & 7248.4 & 1222.511 & 1258.638 & z3e70203t & 6593/STOCKE \\
\hline ESO141-G55 & z3e70206t & 08/15/96 & Yes & 7248.4 & 1222.542 & 1258.662 & z3e70205t & 6593/STOCKE \\
\hline ESO141-G55 & z3i70105t & $10 / 09 / 96$ & Yes & $9085.0^{\mathrm{b}}$ & 1228.710 & 1265.920 & z3i7010ct & 6451/SAVAGE \\
\hline FAIRALL9 & z26o0208n & 04/09/94 & Yes & 7096.3 & 1231.660 & 1267.750 & z26o020at & 5300/SAVAGE \\
\hline FAIRALL9 & z3e70404m & 08/01/96 & Yes & 13407.0 & 1219.702 & 1255.843 & z3e70403t & 6593/STOCKE \\
\hline FAIRALL9 & z3e70406t & 08/01/96 & Yes & 6412.0 & 1240.312 & 1276.384 & z3e70405t & 6593/STOCKE \\
\hline $\mathrm{H} 1821+643$ & z15f0208m & $04 / 17 / 93$ & Yes & 6386.7 & 1231.660 & 1267.750 & $\mathrm{z} 15 \mathrm{f0207t}$ & 4094/SAVAGE \\
\hline $\mathrm{H} 1821+643$ & $\mathrm{z} 27 \mathrm{n} 0108 \mathrm{~m}$ & $04 / 05 / 94$ & Yes & 9123.8 & 1231.660 & 1267.750 & z27n0107t & $5299 / \mathrm{LU}$ \\
\hline IZW1 & z1a60404n & 09/09/93 & No & 24178.1 & 1221.401 & 1257.544 & z1a60403t & 3584/STOCKE \\
\hline IZW1 & z2ia0204n & $02 / 07 / 95$ & Yes & 21897.2 & 1221.299 & 1257.437 & z2ia0203t & 5715/STOCKE \\
\hline MARK279 & z3e70304t & $01 / 17 / 97$ & Yes & 5702.4 & 1222.537 & 1258.673 & z3e70303t & 6593/STOCKE \\
\hline MARK279 & z3e70306t & $01 / 17 / 97$ & Yes & 5702.4 & 1222.576 & 1258.703 & z3e70305t & 6593/STOCKE \\
\hline MARK279 & z3e70308t & $01 / 17 / 97$ & Yes & 5702.4 & 1222.615 & 1258.737 & z3e70307t & 6593/STOCKE \\
\hline MARK279 & z3e7030at & $01 / 17 / 97$ & Yes & 1419.3 & 1222.643 & 1258.762 & z3e70309t & 6593/STOCKE \\
\hline MARK290 & z3kh0104t & $01 / 10 / 97$ & Yes & 1622.0 & 1231.760 & 1268.950 & z3kh0103t & 6590/WAKKER \\
\hline MARK290 & z3kh0105t & $01 / 10 / 97$ & Yes & 2433.0 & 1231.780 & 1268.970 & z3kh0103t & 6590/WAKKER \\
\hline MARK290 & $\mathrm{z} 3 \mathrm{kh} 0107 \mathrm{t}$ & $01 / 10 / 97$ & Yes & 2230.3 & 1231.810 & 1268.990 & z3kh0106t & 6590/WAKKER \\
\hline MARK335 & z1a60304n & $09 / 03 / 93$ & No & 13787.1 & 1221.408 & 1257.550 & z1a60303t & 3584/STOCKE \\
\hline MARK421 & z2ia0104t & $02 / 02 / 95$ & Yes & $9085.0^{\mathrm{C}}$ & 1221.426 & 1257.569 & z2ia0103t & 5715/STOCKE \\
\hline MARK501 & z1a65204m & $02 / 28 / 93$ & No & 29196.3 & 1221.418 & 1257.561 & z1a65203t & 3584/STOCKE \\
\hline MARK509 & z1790208m & $05 / 23 / 93$ & No & 6082.6 & 1231.730 & 1267.840 & z1790207t & 3463/SAVAGE \\
\hline MARK509 & z3e70704t & $10 / 19 / 96$ & Yes & 4257.8 & 1219.468 & 1255.610 & z3e70703t & 6593/STOCKE \\
\hline MARK817 & z3e70104t & $01 / 12 / 97$ & Yes & 8870.4 & 1222.568 & 1258.704 & z3e70103t & 6593/STOCKE \\
\hline MARK817 & z3e70106t & $01 / 12 / 97$ & Yes & 8236.8 & 1222.616 & 1258.741 & z3e70105t & 6593/STOCKE \\
\hline MARK817 & z3e70108t & $01 / 12 / 97$ & Yes & 7907.3 & 1222.645 & 1258.767 & z3e70107t & 6593/STOCKE \\
\hline PKS2155-304 & z1aw0106t & $05 / 11 / 93$ & No & 1558.7 & 1222.580 & 1258.700 & z1aw0105t & 3965/BOGGESS \\
\hline PKS2155-304 & z1aw0107t & $05 / 11 / 93$ & No & 1558.7 & 1222.590 & 1258.710 & z1aw0105t & 3965/BOGGESS \\
\hline PKS2155-304 & z1aw0108m & $05 / 11 / 93$ & No & 1520.6 & 1222.600 & 1258.720 & z1aw0105t & 3965/BOGGESS \\
\hline PKS2155-304 & z3e70504t & $10 / 06 / 96$ & Yes & 3801.6 & 1257.580 & 1293.628 & z3e70503t & 6593/STOCKE \\
\hline PKS2155-304 & z3e70505t & $10 / 06 / 96$ & Yes & 3168.0 & 1257.634 & 1293.669 & z3e70503t & 6593/STOCKE \\
\hline $\mathrm{Q} 1230+0115$ & z3cj0105t & $07 / 11 / 96$ & Yes & 5474.3 & 1216.960 & 1254.200 & z3cj0103t & 6410/RAUCH \\
\hline $\mathrm{Q} 1230+0115$ & z3cj0108t & $07 / 11 / 96$ & Yes & 5474.3 & 1217.000 & 1254.230 & z3cj0106t & 6410/RAUCH \\
\hline
\end{tabular}

${ }^{a}$ HST Proposal Id number (PID) / HST Principal Investigator (PI).

${ }^{b}$ An HST anomaly caused the final exposure to be cut short by 38.8 seconds.

${ }^{c}$ Subexposures 5 through 15 were unusable due to an HST pointing error. The originally scheduled observation was 14598.1 seconds in length. 
Table 2: HST GHRS/G160M Targets

\begin{tabular}{|c|c|c|c|c|c|c|c|c|c|c|}
\hline Target & $\begin{array}{l}\text { RA (J2000) } \\
\text { (hh:mm:ss) }\end{array}$ & $\begin{array}{l}\text { DEC (J2000) } \\
( \pm \text { dd:mm:ss) }\end{array}$ & $\begin{array}{l}l \\
\left({ }^{\circ}\right)\end{array}$ & $\begin{array}{c}b \\
\left({ }^{\circ}\right)\end{array}$ & $\bar{z}$ & $\begin{array}{r}V_{\mathrm{lsr}}^{\mathrm{a}}(\mathrm{H} \mathrm{I}) \\
(\mathrm{km} / \mathrm{s})\end{array}$ & $\begin{array}{c}\Delta V_{\mathrm{lsr}}{ }^{\mathrm{b}} \\
(\mathrm{km} / \mathrm{s})\end{array}$ & $\mathrm{O}^{\mathrm{c}}$ & $\begin{array}{c}\mathrm{V}_{\text {hvc }}{ }^{\mathrm{d}} \\
(\mathrm{km} / \mathrm{s})\end{array}$ & $\overline{\mathrm{S} / \mathrm{N}}$ \\
\hline$(1)$ & $(2)$ & $(3)$ & $(4)$ & $(5)$ & $(6)$ & $(7)$ & $(8)$ & $(9)$ & $(10)$ & $(11)$ \\
\hline $3 \mathrm{C} 273$ & 122906.8 & +020307.8 & $\begin{array}{l}-70.05 \\
\end{array}$ & 64.36 & 0.1583 & -5 & 10.5 & 13 & 115 & 27.2 \\
\hline AKN120 & 051611.4 & -000859.4 & -158.31 & -21.13 & 0.0331 & 5 & -2.7 & $14^{*}$ & -116 & 22.2 \\
\hline ESO141-G55 & 192114.3 & $\begin{array}{lll}-58 & 40 & 14.9\end{array}$ & -21.82 & -26.71 & 0.0371 & 0 & -2.6 & $20^{*}$ & 200 & 25.8 \\
\hline FAIRALL9 & 012345.9 & -584820.9 & -64.93 & -57.83 & 0.0461 & -6 & -8.8 & $20^{*}$ & 190 & 32.7 \\
\hline $\mathrm{H} 1821+643$ & 182157.1 & +642036.7 & 94.00 & 27.42 & 0.2968 & -1 & 46.5 & 6 & -180 & 20.0 \\
\hline IZW1 & 005334.9 & +124136.3 & 123.75 & -50.17 & 0.0607 & -5 & 33.3 & 14 & -100 & 13.2 \\
\hline MARK279 & 135303.4 & +691829.6 & 115.04 & 46.86 & 0.0306 & 5 & 50.0 & $25^{*}$ & -135 & 30.0 \\
\hline MARK290 & 153552.4 & +575409.6 & 91.49 & 47.95 & 0.0296 & -5 & 27.9 & $9^{*}$ & -120 & 17.1 \\
\hline MARK335 & 000619.5 & +201210.3 & 108.76 & -41.42 & 0.0256 & -3 & 30.5 & $25^{*}$ & -400 & 33.3 \\
\hline MARK421 & 110427.4 & +381230.8 & 179.83 & 65.03 & 0.0300 & -8 & 38.2 & 5 & -110 & 23.9 \\
\hline MARK501 & 165352.2 & +394536.6 & 63.60 & 38.86 & 0.0337 & 0 & 55.4 & 9 & -115 & 15.3 \\
\hline MARK509 & 204409.8 & -104324.5 & 35.97 & -29.86 & 0.0344 & 4 & 15.1 & $23^{*}$ & -240 & 28.4 \\
\hline MARK817 & 143622.1 & +584739.4 & 100.30 & 53.48 & 0.0313 & -2 & 19.6 & $27^{*}$ & -115 & 42.7 \\
\hline PKS2155-304 & 215852.0 & -301332.3 & 17.73 & -52.25 & 0.1165 & -4 & 17.5 & 4 & -200 & 20.3 \\
\hline PKS2155-304 & 215852.0 & $\begin{array}{lll}-30 & 13 & 32.2\end{array}$ & 17.73 & -52.25 & 0.1165 & -4 & 3.0 & 3 & -200 & 34.2 \\
\hline $\mathrm{Q} 1230+0115$ & 123050.0 & +011521.7 & -68.74 & 63.66 & 0.1170 & -5 & 1.1 & 7 & 115 & 9.2 \\
\hline
\end{tabular}

${ }^{a}$ The velocity centroid (in $\mathrm{km} \mathrm{s}^{-1}$ ), relative to the LSR, of the Galactic $\mathrm{H}$ I along this sightline as determined from the Leiden/Dwingeloo or Parkes multibeam survey.

${ }^{b} \Delta V_{\mathrm{lsr}}=V_{\mathrm{lsr}}-V_{\mathrm{obs}}$, as determined from the location of the S II Galactic lines (1250, 1253, and $\left.1259 \AA\right)$.

${ }^{c}$ The order of the polynomial used to normalize the spectrum. An asterisk (*) indicates that a broad Gaussian component was included to model intrinsic Ly $\alpha$ emission, present in the observed band.

${ }^{d}$ LSR velocity in $\mathrm{km} \mathrm{s}^{-1}$ of the dominant HI-HVC in this direction as reported by Wakker \& van Woerden (1991).

${ }^{e}$ Values for Pre-COSTAR exposure covering 1222-1258 A.

${ }^{f}$ Values for Post-COSTAR exposure covering 1257-1294 A. 


\subsection{GHRS Calibration}

All of our HST/G160M spectra were taken with the Large Science Aperture (LSA) and are a mixture of pre- and post-COSTAR observations as noted in Table 1. All data were re-calibrated with the final GHRS recommendations (Sherbert \& Hulbert 1997), using the familiar standard IRAF/STSDAS/CALHRS environment, with the following exceptions:

1. Background Subtraction: We find that the null order polynomial (identified in CALHRS nomenclature as PLY_CORR) background subtraction gives superior results to the median (MDF_CORR) and mean (MNF_CORR) background options, mainly due to the inappropriately small size of the default filter box.

2. Wavelength Calibration: As listed in Table 1, the indicated GHRS spectrum Y-balances (SPYBAL's) were used during the recalibration process to correct the initial wavelength zero point offsets.

3. Blemishes and Degradation Accounting: To avoid any possible detector artifacts from appearing as weak spectral features, photocathode blemishes, dead, noisy or "flaky" diodes, pixels with Reed-Solomon decoding errors (which indicate possible data transmission errors) are removed from the spectra. For this reason, some spectra have incomplete wavelength coverage, while others have varying $\mathrm{S} / \mathrm{N}$ ratios across the spectrum.

All subsequent data reductions were performed using the Interactive Data Language (IDL) of Research Systems, Inc (http://www.rsinc.com). Because of the low $\mathrm{S} / \mathrm{N}$ of the individual data files, the subexposure coadditions were performed in wavelength space weighted by exposure time, and not by using the IRAF default STSDAS/CALHRS routines poffsets and specalign. To achieve this, an exposure time vector was created that defines the actual on-target exposure time for each pixel. The exposure time for each sub-exposure is calculated from the information in the FITS header. Specifically, for each exposure, the following procedure was followed:

1. If the spectra were taken in FP-SPLIT mode, coadd all spectra at identical carrousel positions by exposure time.

2. Check the quality of the sub-exposures for anomalies.

3. Recalculate the exposure time from the dataset headers and compare this to the STDSAS/FITS EXPTIME header keyword.

4. If the spectral subexposures were not quarter-stepped, resample the spectra to quarterstepped (quarter GHRS diode) pixels, conserving flux and adjusting the error vectors accordingly.

5. Smooth the spectra to the resolution appropriate for the line-spread function (LSF) of the detector at the time of observation. The pre-COSTAR large science aperture (LSA) line spread 
function (LSF) can be represented by a core of Full Width Half Maximum (FWHM)=1.2 diodes $\left(\sim 20 \mathrm{~km} \mathrm{~s}^{-1}\right.$ at $\left.1240 \AA\right)$ and broad wings (Gilliland \& Hulbert 1993). The postCOSTAR GHRS LSF is well-modeled (Gilliland et al. 1992) by a single sharp Gaussian with FWHM $=1.1$ diodes or $\sim 19 \mathrm{~km} \mathrm{~s}^{-1}$ in our wavelength range. The smoothing is performed by convolving the LSF of the GHRS/LSA at the time of observation with the flux and error vectors.

Once all the subexposures are in place, we used the following procedure to determine the final spectrum:

1. Use the first subexposure of the first dataset to define the wavelength scale.

2. Populate the final flux, error and exposure time vectors with the first subexposure values, except where any anomaly appears in the calibration flags. Possible anomalies include photocathode blemishes, faulty diodes, and decoding errors as previously described.

3. Coadd the next exposure, weighting by the exposure time in the flux vector. Add the exposure times to the exposure vector. Assume Poisson statistics and combine, in quadrature, the exposure's $1 \sigma$ error vector into the merged error vector. The coaddition is performed pixel by pixel into the closest wavelength bin of the first exposure. If no wavelength bin is detected within one-half of a quarter-stepped pixel width, a new wavelength bin is created and populated. This most commonly occurs at the edges of the spectra, as the coverage is extended by multiple observations.

4. Repeat until all exposures of this dataset are merged.

5. If the flux level of the target is constant, or if the temporal separation between observations is small, combine all subexposures of all appropriate datasets to the existing merged exposure. If the flux levels between exposures at different epochs are not constant, then we scale the flux level of the subsequent exposures to the first spectrum for each target listed in Table 1. The error vectors of these exposures are also scaled to preserve the proper $\mathrm{S} / \mathrm{N}$ weighting. The scaling is determined by the average fluxes in the overlap region of the exposures. For ESO 141-G55 (HST exposure z3i70105t), Markarian 509 (HST exposure z3e70704t), and Fairall 9 (HST exposures z3e70404m and z3e70406t), we allow a scaling that varies linearly across the overlap region to compensate for any "Baldwin Effect". 4 For PKS 2155-304, the pre- and post-COSTAR observations covered different wavelength ranges and are analyzed separately.

\footnotetext{
${ }^{4}$ The "Baldwin Effect", first noted in Baldwin (1977), pertains to the observed systematic decrease of the equivalent width of AGN line emission (e.g., Ly $\alpha$ ) with an increase in continuum luminosity. This changes the continuum slope in the vicinity of the Ly $\alpha$ emission in the indicated spectra.
} 


\subsection{Spectral Processing}

\subsubsection{Line Identification: Local Continuum Method and b-values}

To begin the spectral processing, we treat all spectral fluctuations with $>1 \sigma$ negative departures from the continuum as our initial absorption line list. Linear continuum and Gaussian features (with observed Doppler velocities constrained in the range of $12<b_{\text {obs }}<100 \mathrm{~km} \mathrm{~s}^{-1}$ ) are fitted to a $4 \AA$ region centered on prominent features. This wide range of $b$-values allows us to include all possible absorption lines in these spectra. Gaussian features are fitted to the functional form, $\exp \left[-\left(\lambda-\lambda_{c}\right)^{2} / 2 \Delta \lambda_{\mathrm{D}}^{2}\right]$, where $\lambda_{c}$ is the line center and the observed Doppler widths $\left(b_{\text {obs }}\right)$ are related to the Gaussian widths by $\mathbf{W}_{G}=b_{\text {obs }} / \sqrt{2}$, and $\Delta \lambda_{\mathrm{D}}=(\lambda / c) \mathbf{W}_{G}$. The Ly $\alpha$-value is related to the physical properties of the absorbing gas by :

$$
b=\sqrt{\frac{2 k T}{m_{p}}+V_{\text {turb }}^{2}}=\sqrt{V_{\text {therm }}^{2}+V_{\text {turb }}^{2}},
$$

where $T$ is the temperature of the cloud, $m_{p}$ is the proton mass, $k$ is the Boltzmann constant, and $V_{\text {turb }}$ represents non-thermal turbulent motion of the absorbing gas. We selected Gaussian components because, at the spectral resolution and $\mathrm{S} / \mathrm{N}$ of these observations, we are unable to distinguish between Gaussian and Voigt line profiles at high confidence level, and at the column densities that we are sampling, Gaussian and Lorentzian profiles are virtually indistinguishable. In addition it is unclear whether we are resolving discrete individual absorption clouds or nondiscrete ensembles of neutral hydrogen spread in velocity space. Up to 10 Gaussian components are fitted simultaneously to each region of interest, with features taken from the initial line list. If more than 10 Gaussian components are required, or if the continuum is non-linear, we decrease the wavelength region accordingly until the fitting can proceed. When we encounter obvious asymmetries, features broader than $b_{\mathrm{obs}}=100 \mathrm{~km} \mathrm{~s}^{-1}$, or distinct absorption minima, we count them as additional features and add them to the line list.

We repeat this procedure of adding Gaussian components and refitting all features when we encounter an absorption feature broader than $100 \mathrm{~km} \mathrm{~s}^{-1}$, until all modeled features have $b_{\text {obs }}<100 \mathrm{~km} \mathrm{~s}^{-1}$. Occasionally, we relax this limit to allow strong intrinsic and Galactic features to have $b_{\mathrm{obs}}=100 \mathrm{~km} \mathrm{~s}^{-1}$. This minimizes the number of components in exceptionally broad features that are almost certainly not intervening absorptions. Although we allow absorption features with $b_{\text {obs }} \leq 12 \mathrm{~km} \mathrm{~s}^{-1}$ to remain in the initial absorption line list, they are removed after we perform a continuum normalization, or by our selection of $\mathrm{a} \geq 3 \sigma$ significance cutoff. In addition, we observe broad low-contrast absorption troughs $\left(b_{\text {obs }}>100 \mathrm{~km} \mathrm{~s}^{-1}\right)$ in some of the low $\mathrm{S} / \mathrm{N}$ spectra. We interpret these as continuum features, detector artifacts, or possible nondiscrete absorption features. In these cases, we fit the troughs with Gaussians to maintain the continuum integrity, but we do not count them as Ly $\alpha$ absorption features.

We express the strength of an absorption feature in terms of its observed equivalent width $(\mathrm{EW})$ in $\mathrm{m} \AA$. An accurate determination of the EW depends on an accurate modelling of the 
continuum level in the absence of any absorption. For the majority of this paper, we are interested in the rest-frame equivalent widths, $\mathcal{W}=\mathrm{EW} /(1+z)$. We quote $\mathcal{W}$ in all Tables. However we often use the abbreviation EW in contexts where the difference between observed and rest-frame values are unimportant. We calculate the significance level (SL) of each potential absorber by integrating the S/N per $19 \mathrm{~km} \mathrm{~s}^{-1}$ FWHM resolution element (RE) of the best-fit Gaussian. This SL is different (usually larger) than that indicated by the uncertainty in $\mathcal{W}$, which is derived from the uncertainties of the best-fit Gaussian components. Features with $S L \geq 4 \sigma$ are termed "definite" absorbers, while features with $3 \sigma \leq S L<4 \sigma$ are termed "possible" absorbers. The continuum and absorption feature fits are performed in IDL using a Levenberg-Marquardt $\chi^{2}$ minimization implementation based on MINPACK-1 (Moré \& Wright 1993; Markwardt 1999).

The measured $b$-values are independent of the EW measurements and are important determinations in their own right. These are the first $b$-value measurements published at low- $z$, and are essential for converting $\mathcal{W}$ to column density in higher column density absorbers. To obtain $b$-values, the measured Gaussian widths $\left(\mathbf{W}_{G}\right)$ are deconvolved using the smoothed LSFs of the pre- and post-COSTAR data (as appropriate) since the data themselves have been smoothed as described in the previous section. In the limit of very high $\mathrm{S} / \mathrm{N}$ data, this smoothing could decrease our ability to determine accurate $b$-values for very narrow features. But, for the modest $\mathrm{S} / \mathrm{N}$ present in the current data, two effects "trade-off" against each other to make small $b$-values equally accurate in smoothed and unsmoothed data: The error inherent in fitting the data with a Gaussian and the error in determining whether that Gaussian is distinctly broader than the LSF. We see no evidence that smoothing these data have made it more difficult to measure very narrow $b$-values; indeed a couple of $b$-values at $\leq 15 \mathrm{~km} \mathrm{~s}^{-1}$ are found. As Lu et al. (1996) have pointed out on the basis of simulations using moderate $\mathrm{S} / \mathrm{N}$ ratio spectra, these very low- $z b$-values have large errors and are underestimates of the true line widths due to the presence of noise. Specifically, since the data are inherently smeared by the instrumental profile, and by our smoothing, both

with $\sigma_{\text {gauss }} \sim 8.07 \mathrm{~km} \mathrm{~s}^{-1}$, our measured $b$-values are related to our smoothed observed $b$-values $\left(b_{\mathrm{obs}}=\sqrt{2} \mathbf{W}_{G}\right)$ by:

$$
b=\sqrt{2} \sqrt{\mathbf{W}_{G}^{2}-2 \times\left(8.07 \mathrm{~km} \mathrm{~s}^{-1}\right)^{2}}=\sqrt{b_{\mathrm{obs}}^{2}-\left(16.14 \mathrm{~km} \mathrm{~s}^{-1}\right)^{2}} .
$$

As discussed in Paper II, the measured $b$-values in pre-COSTAR data are occasionally artificially large due to spacecraft "jitter". In all Tables reporting $b$-values, we always list the $b$-values corrected by equation 2 above.

\subsubsection{Continuum Normalization: Global Continuum Method}

Because a major source of uncertainty in all EW determinations is the continuum placement, we also performed a global continuum normalization to give two independent estimates ("local" vs. "global") of the continuum placement. We used polynomial fits to model a global continuum and normalize each spectrum. The order of the continuum-fitting polynomial was selected by 
performing F-tests on increasing polynomial orders until adding an additional order is not justified (Mandel 1984). For each spectra, the order of the normalizing polynomial is indicated in Table 2 and is restricted to be less than 30. For objects with broad intrinsic Ly $\alpha$ emission (Akn 120, ESO 141-G55, Fairall 9, Mrk 279, Mrk 290, Mrk 335, Mrk 509, and Mrk 817), we include a single Gaussian to improve the continuum fit near the emission feature. The continuum fits are made on the raw data, with $3 \times \mathrm{FWHM}$ (Full Width at Half Maximum) regions centered on each $3 \sigma$ absorption feature replaced by continuum estimates plus added Gaussian noise. Including noise in the replaced spectral regions was necessary to avoid biasing the global continuum modeling. The unnormalized spectra of each target and the polynomial fits are shown in the spectral plots (Appendix A).

From the difference in the measured values of EW obtained from the "local" and "global" continuum methods, we conservatively overestimate the contribution of the continuum uncertainty to the EW and significance level (SL) determinations. In Figure 1, we compare the deviations of EWs using the local continuum fits from the global continuum results. The differences are well-fitted by a Gaussian with a HWHM (half width at half maximum) of $1.1 \%$. Taking this value to be the $1 \sigma$ error estimate, we conservatively add a $3 \sigma(3.4 \%)$ additional continuum placement uncertainty in quadrature with the statistical uncertainties of the EW, and hence the significance levels, of all features. Note that this systematic continuum placement error is an additional uncertainty above and beyond the statistical uncertainty of absorption feature plus continuum included in the error estimates. Although it is clear from Figure 1 that our continuum normalization correction is a considerable overestimate for most absorption features, a few words are needed to explain the "outliers" at $>10 \%$ difference in Figure 1. These outliers occur mostly from blended lines or clustered absorption features located on the wings of significant continuum features, such as the target's Ly $\alpha$ emission. Multi-component fits of blended absorption lines find a $\chi^{2}$ solution that shares the EW between the absorption components. This increases the uncertainty in determining the EW of the individual blended components, but not the total EW. Even slight continuum adjustments can significantly alter the EW absorption ratio between the blended features, while leaving the total absorption almost unchanged. This produces outliers in Figure 1 that are not associated with the continuum normalization error, but rather arise from special individual circumstances. Hence, our procedure does not underestimate the total errors for these outlier features. Indeed, because a

continuum placement error is rarely included in the error budget for absorption-line EWs, we have been conservative, but we believe correct, in including this additional uncertainty.

\subsubsection{Wavelength Adjustment to Local Standard of Rest (LSR)}

Once all features have been modeled, we use the wavelength of the strongest components of the Galactic S II triplet, $1250.584 \AA, 1253.811 \AA, 1259.519 \AA$ to determine the offset $\Delta V_{\mathrm{lsr}}=$ $\left(V_{\text {lsr }}-V_{\text {obs }}\right)$ that can be applied to adjust the observed, approximately heliocentric, wavelength scale of the GHRS spectra to the LSR frame. These adjustments are given in column 10 of Table 2. This 

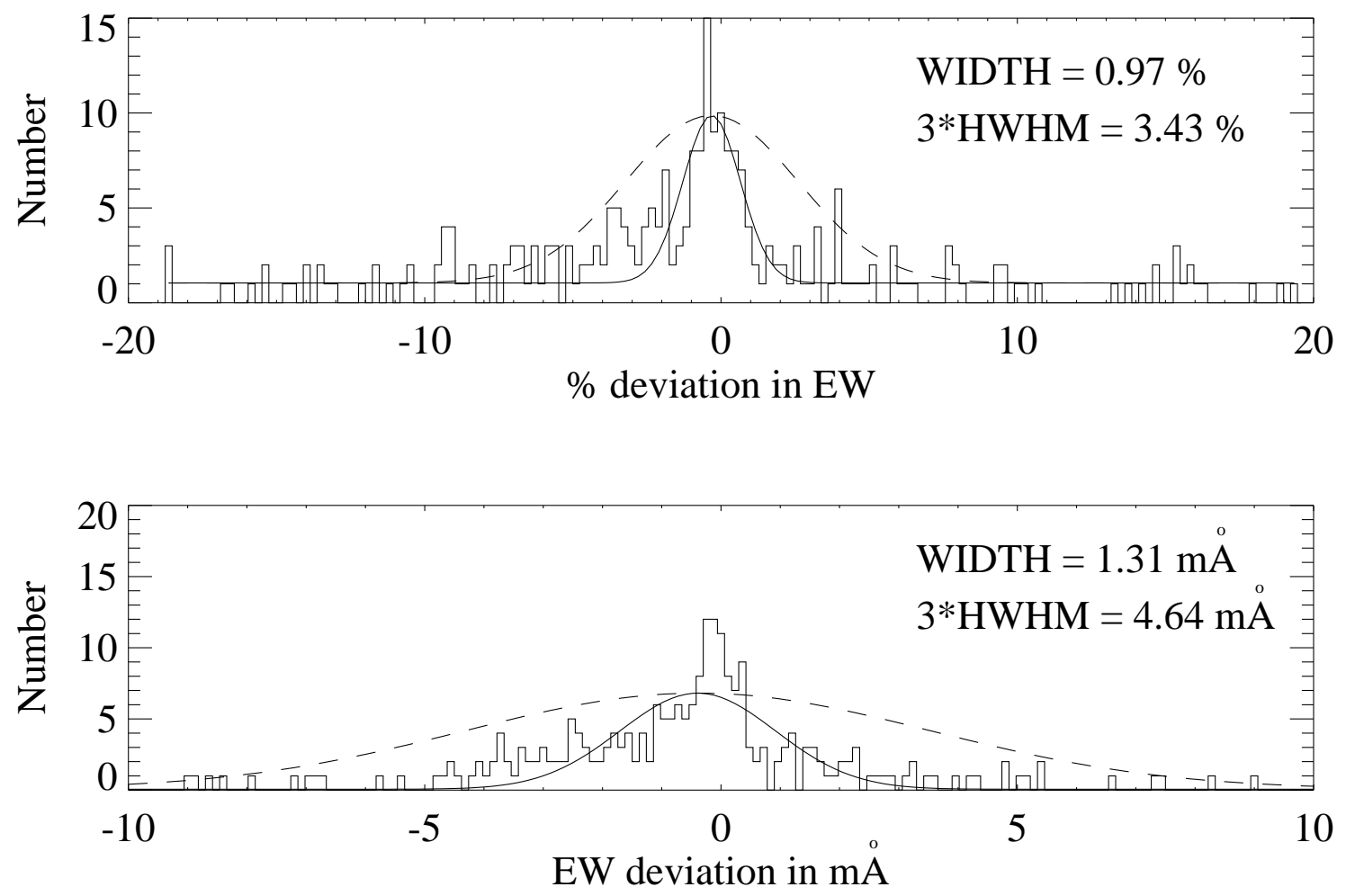

Fig. 1.- The upper panel shows the percent deviation in absorption feature EWs of the "local" continuum determinations versus the "global" determinations. WIDTH indicates the width of the Gaussian that best fits the distribution (shown as a solid curve). The dashed curve represents a Gaussian with a width of $3 \times$ the HWHM (half width at half maximum) of the best-fit distribution. This $3 \sigma$ deviation represents the additional systematic EW uncertainty introduced in the spectral normalization. The bottom panel shows the same deviations in units of $m \AA$. 
technique assumes that the S II lines occur at the same LSR velocity as the dominant component of the Galactic H I, determined from the Leiden/Dwingeloo survey (Hartmann \& Burton 1997) for all objects except Fairall 9 and ESO 141-G55. For these two objects, the Galactic H I velocities relative to the LSR were determined from the Parkes multibeam survey (Haynes et al. 1999).

The Galactic H I velocities relative to the LSR along each sightline are given in column 9 of Table 2. It is our opinion that this LSR-corrected wavelength scale is more accurate than the heliocentric scale provided by the GHRS recalibration because the precise location of the target in the LSA is unknown. Owing to target motion in the LSA, pre-COSTAR observations can have velocity offsets as large as $70 \mathrm{~km} \mathrm{~s}^{-1}$ (1" in the LSA), while post-COSTAR observations can have velocity offsets as large as $60 \mathrm{~km} \mathrm{~s}^{-1}\left(0.85^{\prime \prime}\right.$ in the LSA). As described in the HST Data Handbook $^{5}$, there are a number of other effects, such as thermal motion, that can significantly affect the wavelength scale of an observation. By consistently applying our LSR adjustment, we obtain a more precise wavelength scale. We estimate that the accuracy with which H I and S II velocities can be matched is $\pm 5 \mathrm{~km} \mathrm{~s}^{-1}$, limited by the width and structure of the $\mathrm{H}$ I $21 \mathrm{~cm}$ profile. This amount of velocity uncertainty is added in quadrature to the individual uncertainties of the features based upon the Gaussian fits. This total velocity uncertainty of individual absorption features is $\leq 10 \mathrm{~km} \mathrm{~s}^{-1}$, and it is this total value which is listed in all tables. Column 8 of Table 2 lists these velocity offsets for each target.

We construct the final line list by refitting all features in the manner described above in the normalized ("global" continuum method) LSR. We use the Gaussian centers, heights, and widths $\left(\mathbf{W}_{G}\right.$, related to the $b$-value) from the "local" continuum method as the initial values for the final $\chi^{2}$ "global continuum" minimization to determine the final line lists. As described above, we determine the statistical significances of all lines using an error estimate that includes both the statistical error in the $\chi^{2}$ fitting technique and the systematic error associated with continuum placement.

\subsubsection{Galactic Spectral Features}

The 1218-1300 Å range contains only a few strong Galactic interstellar lines (see Table 3, Morton 1991). These lines, although useful for correcting the wavelength scale to LSR, also eliminate a small portion of our available pathlength for Ly $\alpha$ detection. A full discussion of the pathlength availability appears in Appendix A. Table 3 lists the rest wavelength for each of the Galactic lines in our waveband, as well as $\log (\lambda f)$ and $\lambda f$. Unsaturated lines of the same species and ionization state are expected to have EW ratios that match the $\lambda f$ ratios. For example, $\mathrm{N} \mathrm{V} \lambda 1238$ and $\lambda 1242$ are expected to have EWs in the ratio of $2: 1$. These ratios allow for distinction between Galactic absorption and intervening Ly $\alpha$ absorption at expected Galactic line positions. For many of these features, the appearance of multiple lines of the same species in our waveband allows us

\footnotetext{
${ }^{5}$ Version 3.1: March 1998, Section 37.6.5, "Wavelength Data Uncertainty", available from http://www.stsci.edu .
} 
to identify Galactic features based upon expected wavelengths and strengths. These features are denoted by a "g:" in the line lists. Since highly ionized species may have different velocities within our Galaxy, we allow an offset of up to $0.2 \AA\left(\sim 50 \mathrm{~km} \mathrm{~s}^{-1}\right)$ for definite $(S L \geq 4 \sigma)$ Galactic feature identifications. Possible $(3 \sigma \leq S L<4 \sigma)$ Galactic identifications are allowed a larger offset range up to $0.5 \AA\left(\sim 120 \mathrm{~km} \mathrm{~s}^{-1}\right)$ and are denoted by a question mark in the line identifications.

Often, Galactic absorption is detected at velocities other than the LSR, due to well-known high-velocity clouds (HVCs) within our Galaxy. These detections are indicated by a "h:" in the identification field of our line lists. Our observations are unique in that they are able to place upper limits on metallicity of the HVCs in several species, Mg II, S II, and, in some cases, Si II. Individual detections of HVCs will be reported both in the line lists and in later sections. A detailed analysis of the HVC results toward Complex C and the Magellanic Stream will appear elsewhere (Gibson et al. 2000a,b).

Galactic and HVC absorption lines remove a portion of our available pathlength for Ly $\alpha$ detection. At the location of each definite, or possible, Galactic and HVC absorption line, we remove a portion of the spectrum equal to the FWHM of the absorption feature on each side of the identified line center. We suspect that occasional weak intervening Ly $\alpha$ features are superimposed upon Galactic/HVC features. However, if we cannot conclusively determine this occurrence based upon expected line strengths, we consider the superimposed portion of the spectrum unusable for Ly $\alpha$ detection. In such cases, we report the total absorption as Galactic/HVC and remove this portion of the available pathlength for intervening Ly $\alpha$ detection.

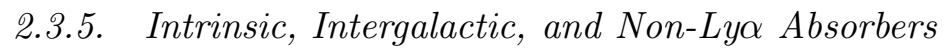

Many AGN are observed to possess "intrinsic" absorbers at $z_{\mathrm{abs}} \sim z_{\mathrm{em}}$. One measure of the appropriateness for excluding potential intrinsic absorption systems from our line list is the "Proximity Effect" (Bajtlik \& Ostriker 1988) or "Inverse Effect" (Murdoch et al. 1986), which refer to the observed decrease of absorption line density, $\mathrm{d} \mathcal{N} / \mathrm{d} z$, when approaching the target AGN's emission redshift. This decrease has been interpreted as due to increased photoionization of the absorption-line systems by the target AGN's UV radiation field (Carswell et al. 1982). To avoid any possible skewing of our statistics by this effect, we include only absorption systems in which the ionizing radiation from the target AGN is $10 \%$ or less of the metagalactic ionization radiation field. At low redshift, the metagalactic ionizing intensity at $912 \AA$ has been estimated to be $I_{0}=1.3_{-0.5}^{+0.8} \times 10^{-23} \mathrm{ergs} \mathrm{cm}{ }^{-2} \mathrm{~s}^{-1} \mathrm{~Hz}^{-1} \mathrm{sr}^{-1}$ (Shull et al. 1999b). Assuming that the mean value of the specific ionizing luminosity at $912 \AA$ from the IUEAGN database, $L_{912}=6 \times 10^{29} \mathrm{erg}$ $\mathrm{s}^{-1} \mathrm{~Hz}^{-1}$, is representative of our HST targets, we can calculate the "standoff" distance $(D)$ from the target QSO at which the QSO's ionizing flux decreases to $I_{0} / 10$ :

$$
\frac{L_{912}}{\left(4 \pi D^{2}\right) 4 \pi}=\frac{I_{0}}{10} \Rightarrow D=\frac{1}{4 \pi} \sqrt{\frac{10 L_{912}}{I_{0}}}=17.5 h_{70}^{-1} \mathrm{Mpc}
$$


which is equivalent to $c z \approx 1,200 \mathrm{~km} \mathrm{~s}^{-1}$, where $h_{70}$ is the Hubble constant in units of $70 \mathrm{~km} \mathrm{~s}^{-1}$ $\mathrm{Mpc}^{-1}$.

Therefore, we consider non-Galactic absorption features within $1,200 \mathrm{~km} \mathrm{~s}^{-1}$ of the redshift of the target to be either Ly $\alpha$ clouds near the AGN affected by the "proximity effect" or intrinsic absorption near the target. For consistency, we will use the term "intrinsic" for all Ly $\alpha$ absorptions within $1,200 \mathrm{~km} \mathrm{~s}^{-1}$ of the target, or metal lines associated with the target AGN. Lines identified as Ly $\alpha$ outside of this limit will be termed "intergalactic". Non-Ly $\alpha$ lines associated with known higher- $z$ systems, such as in H 1821+643, will be termed "non-Ly $\alpha$ " absorbers.

Lines intrinsic to the target AGN are not included in any of our low- $z$ Ly $\alpha$ forest statistics and are marked by an "i:" prefix in the identification fields of the target line lists. Likewise, the pathlength within $1,200 \mathrm{~km} \mathrm{~s}^{-1}$ of the AGN is excluded from our total observed pathlength. Our choice of a $1,200 \mathrm{~km} \mathrm{~s}^{-1}$ radial velocity limit relative to the target $\left(\mathrm{V}_{r}\right)$ limit is supported by the analysis of intrinsic absorption lines in Seyfert 1 galaxies by Crenshaw et al. (1999). In Figure 2, we display this sample, obtained from ultraviolet HST/FOS and GHRS Ly $\alpha$, C IV $\lambda \lambda 1548,1550$, N V $\lambda \lambda 1238,1242$, and S IV $\lambda \lambda 1393$, 1402 detections, in terms of number (N) of intrinsic systems versus $\mathrm{V}_{r}$. Five of the 17 targets in Crenshaw et al. (1999) are also in our sample. As shown by the vertical line at $-1,200 \mathrm{~km} \mathrm{~s}^{-1}$ in Figure 2, our radial velocity limit includes the vast majority, but not quite all, of the detected intrinsic systems. These results support the claim that our blueward velocity limit is reasonable, but not perfect, in protecting against any outflowing "intrinsic" absorbers associated with the target. In Figure 3, we plot $\left|\mathrm{V}_{r}\right|$ versus rest-frame $\mathrm{EW}(\mathcal{W})$ for all Ly $\alpha$ absorbers in our sample with $\left|\mathrm{V}_{r}\right|<5,000 \mathrm{~km} \mathrm{~s}^{-1}$. Notice that several absorbers with $\left|\mathrm{V}_{r}\right|<1,200 \mathrm{~km} \mathrm{~s}^{-1}$ have $\mathcal{W}>200 \mathrm{~m} \AA$, while only one of the absorbers identified as intergalactic has $\mathcal{W}>200 \mathrm{~m} \AA$. This further supports our assumption that a $1,200 \mathrm{~km} \mathrm{~s}^{-1}$ blueward velocity limit accounts for the majority of intrinsic or outflowing absorbers. In terms of pure Hubble flow distance, this blueward velocity limit is well in excess of the $230 h_{70}^{-1} \mathrm{kpc}$ galactic halo size inferred by Lanzetta et al. (1995), and probably eliminates any possible Ly $\alpha$ absorptions associated with the targets.

We stress that any velocity limit that purports to separate intergalactic from intrinsic systems is somewhat uncertain. While we have presented several pieces of evidence that $1,200 \mathrm{~km} \mathrm{~s}^{-1}$ is a reasonable velocity limit, it is impossible unambiguously to classify any individual Ly $\alpha$ absorber as either intrinsic or intergalactic. Any use of the intrinsic or intergalactic line lists must take this uncertainty into account.

A few of our targets, such as H 1821+643, have known intergalactic Ly $\alpha$ absorption systems with redshifts greater than our survey cutoff. Higher Lyman series lines or metal-line absorptions associated with these systems that fall within our wavelength range (1218-1300 $\AA$ ) are termed "non-Ly $\alpha$ ". These features are indicated by a "z:" in the identification field of the line lists. As with the intrinsic features, these features are not included in any of our low- $z$ Ly $\alpha$ forest statistics. Additionally, the pathlength obscured by these features is excluded from the available pathlength 
for Ly $\alpha$ detection using the same method as the Galactic/HVC features.

\section{Results by Object}

In this section, the observations of each target are discussed in detail. Composite spectra (Figures 5-20) and a spectral line identification table (Table 9) appear in Appendix A, for our sightlines in the same order presented here. The wavelength scales have been corrected to the LSR by equating the Galactic H I LSR velocity in each sightline (Table 2, column 7) with the location of the S II $\lambda \lambda 1250,1253,1259$ Galactic interstellar lines in the observed waveband. To provide an overall sense of the number and strength of our detections, as well as the quality of our spectra, Figure 4 shows the number of reported intergalactic Ly $\alpha$ absorbers as a function of significance level (SL).

\section{1. $3 \mathrm{C} 273$}

The sightline to 3C 273 is perhaps the most studied of all low- $z$ QSO targets (Bahcall et al. 1991a,b, 1993; Brandt et al. 1993, 1997; Morris et al. 1991, 1993; Weymann et al. 1995). Morris et al. (1993) provide a summary of all HST spectra available as of 1993, including those observed with the GHRS+G160M, GHRS+G140L, and FOS+G130. Refitting all features with their JASON software, Morris et al. (1993) report 12 features in our wavelength range of interest $(z<0.07)$. Our spectrum combines the two GHRS G160M spectra summarized in Morris et al. (1993) with

three additional 1218-1250 ̊ spectra obtained in 1994 (see Table 1). This results in a 1218-1300 spectrum shown in Figure 5, the only one in our sample that covers our entire spectral range of interest. Our analysis of these spectra, recalibrated as discussed earlier, reveals 18 Ly $\alpha$ absorption features at $S L \geq 3 \sigma$, of which 13 have $S L \geq 4 \sigma$. Our results differ from those of Morris et al. (1993) in the following regards:

1. At $\lambda \approx 1224.2-1225.0 \AA$ we see a broad absorption trough at the red edge of the Galactic damped Ly $\alpha$ line that we model with two $\operatorname{Ly} \alpha$ absorption features greater than $4 \sigma$ totalling $\sim 64 \mathrm{~m} \AA$. Bahcall et al. (1991a) report a $240 \mathrm{~m} \AA$ feature at $1224.52 \AA$, while Bahcall et al. (1993), Brandt et al. (1993), and Bahcall et al. (1991b) do not report this feature.

2. The $1280.3 \AA$ feature (not given in any other line lists) was determined to be a Ly $\alpha$ absorber, not Galactic C I $\lambda 1280$, owing to the absence of the expected C I $\lambda 1277$ in our spectrum.

Also detected along this sightline are the Galactic features Mg II $\lambda 1239.9, \mathrm{~N}$ V $\lambda \lambda 1238,1242$, S II $\lambda \lambda 1250,1253,1259$, and Si II $\lambda 1260.4+$ Fe II $\lambda 1260.5$ (which is possibly blended with C I $\lambda 1260$ ). Based upon the absence of a Mg II $\lambda 1240.4$ detection, it is possible that the $1240.01 \AA$ detection could be $\operatorname{Ly} \alpha$ instead of the reported $\mathrm{Mg}$ II $\lambda 1239.9$. 


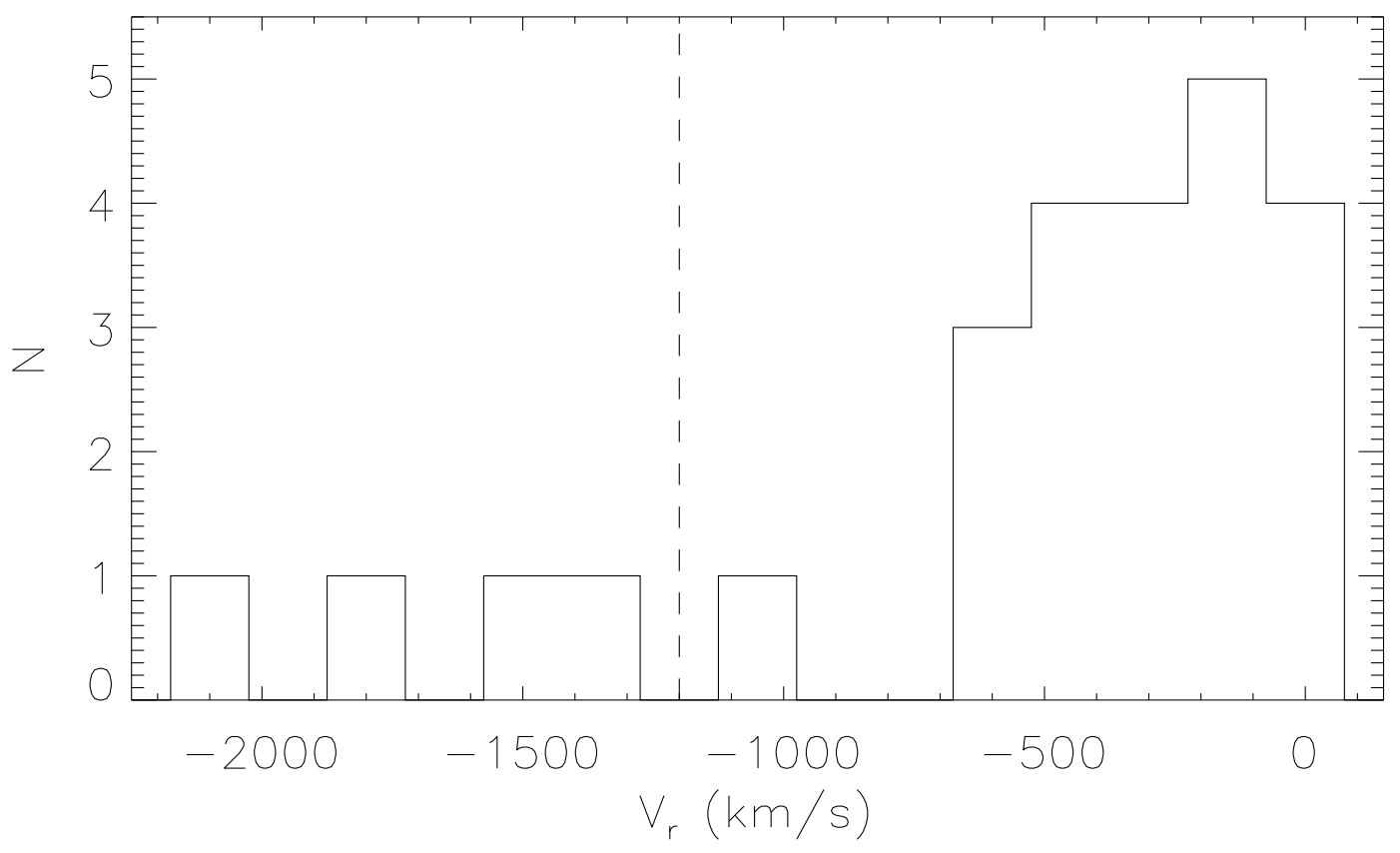

Fig. 2.- Radial velocity distribution relative to the target $\left(\mathrm{V}_{r}\right.$ in $\left.\mathrm{km} \mathrm{s}^{-1}\right)$ of the HST UV sample of Seyfert 1 galaxy "intrinsic" absorbers presented by Crenshaw et al. (1999). The dashed vertical line at $-1,200 \mathrm{~km} \mathrm{~s}^{-1}$ indicates our blueward radial velocity limit for determining which Ly $\alpha$ features are intrinsic or "associated" with the target. 
Table 3. Galactic Lines

\begin{tabular}{lclr}
\hline \hline Ion & Wavelength $(\AA)$ & $\log (\lambda f)$ & \multicolumn{1}{c}{$\lambda f$} \\
\hline N V & 1238.821 & 2.289 & 194.54 \\
Mg II & 1239.925 & -0.106 & 0.784 \\
Mg II & 1240.395 & -0.355 & 0.442 \\
N V & 1242.804 & 1.988 & 97.28 \\
S II & 1250.583 & 0.834 & 6.82 \\
S II & 1253.811 & 1.135 & 13.65 \\
S II & 1259.519 & 1.311 & 20.46 \\
Si II & 1260.422 & 3.104 & 1270.57 \\
Fe II & 1260.533 & 1.499 & 31.55 \\
C I & 1260.736 & 1.696 & 49.66 \\
C I & 1277.245 & 2.091 & 123.31 \\
C I & 1280.135 & 1.493 & 31.12 \\
\hline
\end{tabular}

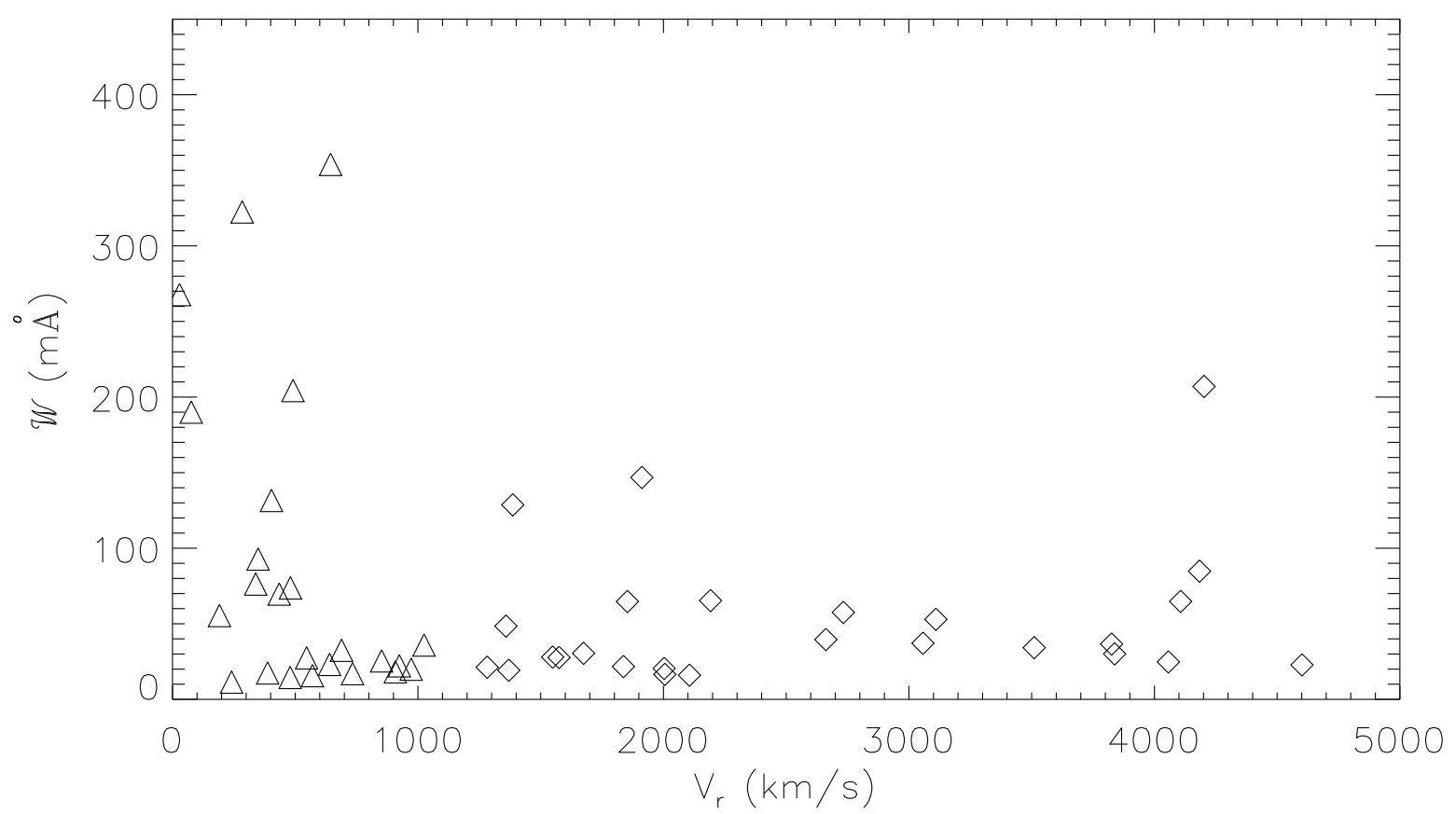

Fig. 3. - Distribution of rest-frame $\mathrm{EW}(\mathcal{W})$ versus radial velocity relative to target $\left(\left|\mathrm{V}_{r}\right|\right.$ in $\left.\mathrm{km} \mathrm{s}^{-1}\right)$ of our sample. The triangle absorbers are those determined by our criterion to be intrinsic $\left(\left|\mathrm{V}_{r}\right|\right.$ $<1,200 \mathrm{~km} \mathrm{~s}^{-1}$ ), while the diamonds indicate intergalactic Ly $\alpha$ absorbers. 


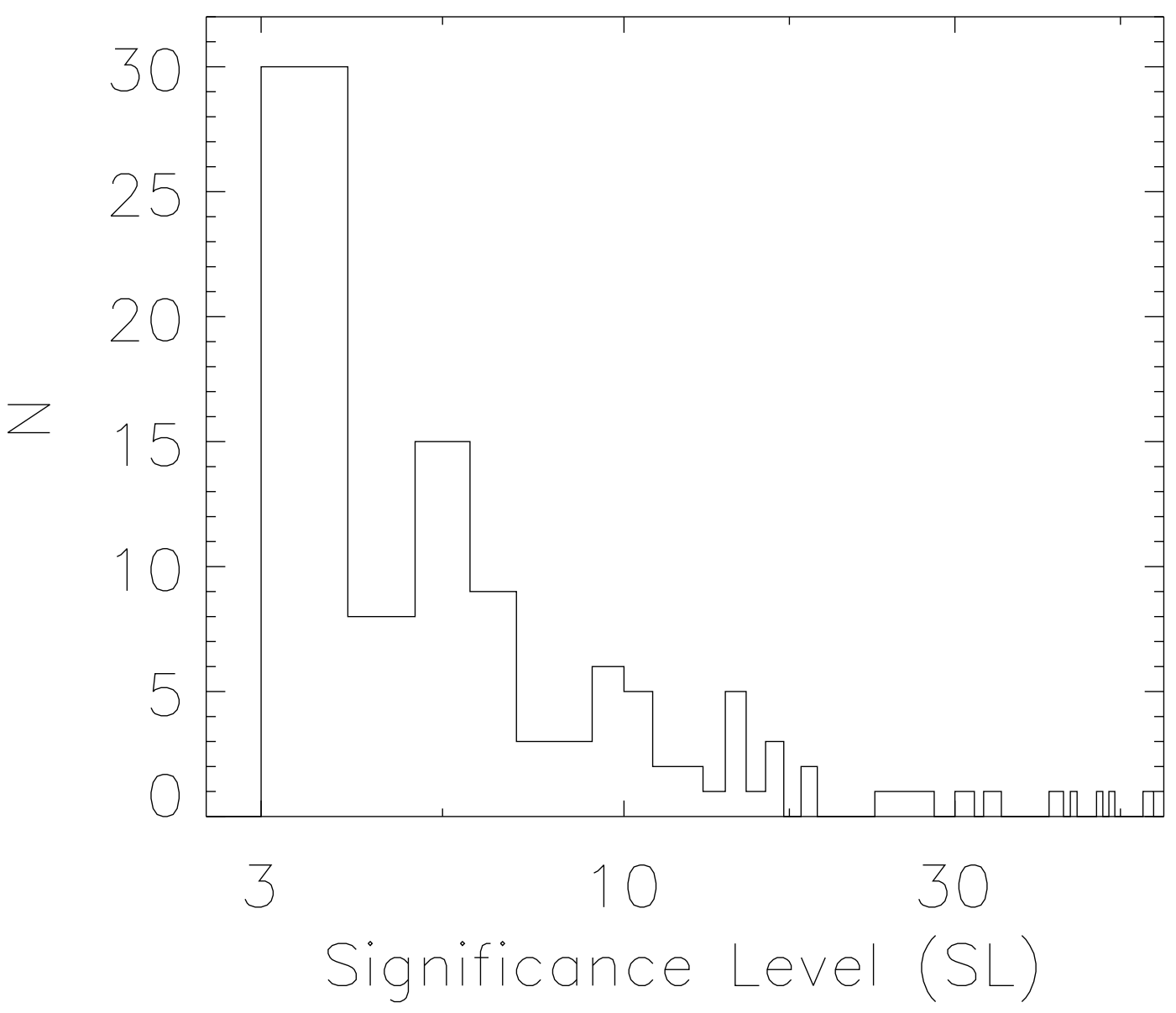

Fig. 4. - Significance level (SL) distribution of our detected intergalactic Ly $\alpha$ absorbers. 
As discussed in Sembach \& Savage (1996) and Burks et al. (1994), the sightline towards 3C 273 passes through the edges of Galactic radio loops I and IV near the North Polar Spur $(l, b)=\left(290^{\circ}\right.$, $64.4^{\circ}$ ) and near the Sagittarius spiral arm. Although no HVCs are reported in this direction, we do detect Galactic N V $\lambda 1238$, S II $\lambda \lambda 1250,1253,1259$, and Si II $\lambda 1260.4+$ Fe II $\lambda 1260.5$ at infalling velocities up to $50 \mathrm{~km} \mathrm{~s}^{-1}$. This is most prevalent in the peculiarly shaped N V $\lambda 1242$ absorption.

In Weymann et al. (1995), the $1220 \AA$ and $1222 \AA$ Ly $\alpha$ features were measured to have $b$-values of $40.7 \pm 3.0 \mathrm{~km} \mathrm{~s}^{-1}$ and $34.3 \pm 3.3 \mathrm{~km} \mathrm{~s}^{-1}$, respectively. They analyzed the same GHRS observations as we did, using Voigt profile fitting on spectra that were carefully cross-correlated at the subexposure level. We elected not to include such cross-correlations in our standard data reduction because the vast majority of our GHRS subexposures were of insufficient $\mathrm{S} / \mathrm{N}$ to accomplish this procedure with confidence. Positional shifts of the target in the HST aperture can cause sub-diode spectral shifts in the subexposures. This causes us occasionally to overestimate the $b$-values of the absorption features, but it does not affect our measurements of the centers, EWs, or significance levels of the absorption features. The consequences of this drift on our measured $b$-values will be explored in Paper II. The LSR offset for this spectrum was $0.044 \AA$ or $10.5 \mathrm{~km} \mathrm{~s}^{-1}$ at $1253 \AA$.

\subsection{Akn 120}

The spectrum of Akn 120 (Arakelian 120, also known as Mrk 1095) covers 1223 to $1259 \AA$ and has an average $\mathrm{S} / \mathrm{N}$ per resolution element of 22. Our spectrum of Akn 120 contains 5 definite and 2 possible Ly $\alpha$ absorbers, one intrinsic Ly $\alpha$ absorption, and strong Galactic detections in Mg II $\lambda \lambda 1239.9,1240.4$, S II $\lambda \lambda 1250,1253$, and N V $\lambda 1238$. Based upon only a $0.9 \sigma$ detection of $\mathrm{N} \mathrm{V} \lambda 1242$, it appears that N V $\lambda 1238$ (which appears extremely broad) is partially contaminated by an intergalactic Ly $\alpha$ system. The $0.9 \sigma \mathrm{N} \mathrm{V} \lambda 1242$ feature is below our limit for inclusion. $\mathrm{N}$ V $\lambda 1242$ is listed as the alternate identification for the $1242.9 \AA$ Ly $\alpha$ feature because its spectral proximity falls within our generous limits (§ 2.3.4). However, due to the strength of the $1242.9 \AA$ feature, we are confident of the identification as Ly $\alpha$. Of special note is the well-resolved and closely spaced $\left(\Delta v=60 \mathrm{~km} \mathrm{~s}^{-1}\right)$ pair of strong Ly $\alpha$ absorbers at 7960 and $8020 \mathrm{~km} \mathrm{~s}^{-1}$. This

pair is flanked to the blueward by two additional $\sim 4 \sigma$ absorbers at 7792 and $7867 \mathrm{~km} \mathrm{~s}^{-1}$. The dominant HVC H I velocity, relative to the LSR, in this direction (Wakker \& van Woerden 1991) is $-116 \mathrm{~km} \mathrm{~s}^{-1}$. We not detect this $\mathrm{HVC}$ in any of the Galactic lines in our waveband. For S II $\lambda \lambda 1250,1253$, we do not detect this HVC at the $4 \sigma \mathcal{W}$ limit of $20 \mathrm{~m} \AA$. The Akn 120 sightline was corrected to the LSR using the Galactic H I and only two S II lines (1250, $1253 \AA$ ). The LSR offset for this spectrum was $-0.011 \AA$ or $-2.7 \mathrm{~km} \mathrm{~s}^{-1}$ at $1253 \AA$. 


\subsection{ESO 141-G55}

Our spectrum of ESO 141-G55 contains no definite absorbers and only one possible intergalactic Ly $\alpha$ absorber. This spectrum has a good $\mathrm{S} / \mathrm{N}(\sim 26$ per RE), so that we are sensitive to $4 \sigma$ Ly $\alpha$ absorptions below $40 \mathrm{~m} \AA$ for a large portion of the spectrum. This sightline does contain strong Galactic N V $\lambda \lambda 1238,1242$, Mg II $\lambda \lambda 1239.9,1240.4$, S II $\lambda \lambda 1250,1253$, 1259, and Si II $\lambda 1260.4+$ Fe II $\lambda 1260.5$. We also detect an HVC at $V_{\mathrm{lsr}} \cong-65 \mathrm{~km} \mathrm{~s}^{-1}$ in S II $\lambda \lambda 1250,1253$, 1259, Si II $\lambda 1260.4+$ Fe II $\lambda 1260.5$, and possibly N V $\lambda \lambda 1238,1242$ (1.7 $\sigma$ and $1.4 \sigma$ respectively). We also detect a very strong intrinsic Ly $\alpha$ absorption at $1258 \AA$, or $V_{\mathrm{lsr}} \cong-640 \mathrm{~km} \mathrm{~s}^{-1}$ relative to ESO 141-G55. This sightline does not pass through any known radio loops or Galactic H IHVCs. The flux level of the z3i70105t HST exposure was scaled to be consistent with the other ESO 141-G55 exposures (see Table 1). The scaling was applied linearly across the overlap region with the other exposures to compensate for a noticeable "Baldwin Effect" between exposures. The LSR offset for this spectrum was $-0.011 \AA$ or $-2.6 \mathrm{~km} \mathrm{~s}^{-1}$ at $1253 \AA$.

\subsection{FAIRALL 9}

We detect 8 definite, 1 possible, and 6 intrinsic Ly $\alpha$ absorption features in our spectrum of Fairall 9. The spectrum of Fairall 9 includes 3 separate post-COSTAR exposures, one of which was reported in Lu, Savage, \& Sembach (1994). The two additional exposures were obtained in 1996, two years after our first exposure. The additional exposures allow us to confirm and refine the earlier results on both the Ly $\alpha$ absorptions and the Galactic/HVC absorptions. The flux levels of the additional HST/G160M exposures, z3e70404m and z3e70406t, were scaled to be consistent with the initial Fairall 9 exposure. The scaling was applied linearly across the overlap region with the initial exposure. The feature identified as $\mathrm{N}$ V $\lambda 1238.8$ in Table 9, is probably blended with a Ly $\alpha$ feature based upon the marginal $1.8 \sigma(\mathcal{W}=8 \pm 6 \mathrm{~m} \AA)$ detection of $\mathrm{N} V \lambda 1242$. Given the expected 2:1 ratio of these Galactic absorption features, the $48 \pm 11 \mathrm{~m} \AA$ absorption feature at $1238.8 \AA$ is anomalously strong and asymmetric. However, given the uncertainty in determining the relative strength and $b$-value for the possible Ly $\alpha$ absorption, we have chosen to exclude this feature and the associated pathlength from our Ly $\alpha$ survey.

The Fairall 9 sightline passes through the Magellanic Stream (MS), which is detected in our waveband (1220-1276 $\AA$ ) in Si II $\lambda 1260.4$ + Fe II $\lambda 1260.5$ and S II $\lambda \lambda 1250,1253$. In addition, the Galactic Si II $\lambda 1260.4+$ Fe II $\lambda 1260.5$ absorption feature appears to be blended with a MS S II $\lambda 1259$ absorption. H I $21 \mathrm{~cm}$ emission (Morras 1983) reveals two high velocity MS components at $\sim 160 \mathrm{~km} \mathrm{~s}^{-1}\left(N_{\mathrm{HI}} \sim 2 \times 10^{19} \mathrm{~cm}^{-2}\right)$ and $200 \mathrm{~km} \mathrm{~s}^{-1}\left(N_{\mathrm{HI}} \sim 6 \times 10^{19} \mathrm{~cm}^{-2}\right)$. Lu, Savage, \& Sembach (1994) report two high velocity components of Si II $\lambda 1526$ at $+170 \mathrm{~km} \mathrm{~s}^{-1}$ and $+200 \mathrm{~km} \mathrm{~s}^{-1}$. In our S II $\lambda \lambda 1250,1253,1259$ detections of the MS, we are unable to resolve the MS clearly into separate components. Savage, Sembach, \& Lu (1997) report detection of this HVC in Si II $\lambda 1260.4+$ Fe II $\lambda 1260.5$. We confirm this detection centered at $+180 \mathrm{~km} \mathrm{~s}^{-1}$ and 
covering the range +100 to $+260 \mathrm{~km} \mathrm{~s}^{-1}$. We were able to model this absorption with two features centered at $V_{\mathrm{lsr}}=+128 \mathrm{~km} \mathrm{~s}^{-1}$ and $+190 \mathrm{~km} \mathrm{~s}^{-1}$. We do not detect the MS in N V $\lambda \lambda 1238$, 1242 or in Mg II $\lambda \lambda 1239.9,1240.4$ at the $10 \mathrm{~m} \AA$ level. Assuming that the MS S II $\lambda \lambda 1250,1253$ absorption features are on the linear portion of the curve of growth, (Gibson et al. 2000b) find a MS metallicity of $[\mathrm{S} / \mathrm{H}]=-0.52 \pm 0.04$. This compares to the LMC and SMC values of $-0.57 \pm 0.09$ and $-0.68 \pm 0.15$, respectively (Russell \& Dopita 1992).

While several absorbers are detected in the wavelength range $1262-1266 \AA$, the rapidly rising continuum on the blue wing of Ly $\alpha$ calls their reality into question. Specifically, due to our concerns about the reality of these features, we have refit the continuum for Fairall 9 by altering the order of the global fit significantly. The statistical significances of these features remains robust despite these changes, so we list them as definite or possible Ly $\alpha$ features as shown in Figure 8.

The z3e70404m exposure of Fairall 9 contains a "medium" photocathode blemish in the 1254$1256 \AA$ range. This blemish, combined with the coaddition of multiple exposures with different wavelength ranges, produces a notch in the sensitivity detection limit near $1255 \AA$. The LSR offset for this spectrum was $-0.037 \AA$ or $-8.8 \mathrm{~km} \mathrm{~s}^{-1}$ at $1253 \AA$.

\section{5. $\mathrm{H} 1821+643$}

The GHRS/G160M spectrum of H 1821+643 (as known as QSO E1821+643) covers 1231.66$1267.75 \AA$ or $0.01315<z<0.04284$. In this spectrum, we detect five definite and seven possible Ly $\alpha$ lines, along with very strong Galactic N V $\lambda \lambda 1238,1242$ and Mg II $\lambda \lambda 1239.9,1240.4$. Savage, Sembach, \& Lu (1995) report C IV-HVCs along this sightline at $V_{\mathrm{lsr}}=-70,-120$, and $-213 \mathrm{~km} \mathrm{~s}^{-1}$ with column densities of $N_{\mathrm{CIV}}=6,6$ and $1 \times 10^{13} \mathrm{~cm}^{2}$, respectively. We detect the $V_{\mathrm{lsr}}=-70 \mathrm{~km} \mathrm{~s}^{-1}$ component in $\mathrm{N} \mathrm{V} \lambda 1238$ and S II $\lambda 1250$, and the $V_{\mathrm{lsr}}=-120 \mathrm{~km} \mathrm{~s}^{-1}$ component in S II $\lambda \lambda 1253,1259$, and Si II $\lambda 1260.4+$ Fe II $\lambda 1260.5$. The individual detection of the -70 and $-120 \mathrm{~km} \mathrm{~s}^{-1}$ components is complicated by their spectral proximity. The LSR offset for this spectrum was $0.187 \AA$ or $44.7 \mathrm{~km} \mathrm{~s}^{-1}$ at $1253 \AA$.

Many Ly $\alpha$ lines outside of our wavelength coverage have been reported along this sightline. This complicates detection of Ly $\alpha$ absorption features in our spectrum due to the possibility of non-Ly $\alpha$ lines associated with the higher $c z \operatorname{Ly} \alpha$ absorbers occurring in our waveband. As reported in Bahcall et al. (1993), Savage, Sembach, \& Lu (1995), and Tripp, Lu, \& Savage (1998a,b), this sightline contains 9 known intergalactic Ly $\alpha$ absorbers with $\mathcal{W}>200 \mathrm{~m} \AA$ that are outside our redshift coverage. Also outside our coverage are 19 weaker intergalactic Ly $\alpha$ absorbers. Six of these have $100 \mathrm{~m} \AA \leq \mathcal{W}<200 \mathrm{~m} \AA$ and 13 with $50 \mathrm{~m} \AA \leq \mathcal{W}<100 \mathrm{~m} \AA$. In addition to these 28 Ly $\alpha$ systems, Savage, Sembach, \& Lu (1995) report a Ly $\beta$ absorber at $z=0.21656$. We doubt the reality of this identification owing to the absence of any Ly $\alpha$ absorption at this redshift $(1478.9 \AA)$ in Tripp, Lu, \& Savage (1998a,b). We do detect the feature identified as Ly $\beta$ in Savage, Sembach, \& Lu (1995) at $1247.94 \AA$, but we identify it as Ly $\alpha$ at $\mathrm{z}=0.0265$. 
Bahcall et al. (1993) report a $680 \mathrm{~m} \AA$ intrinsic Ly $\alpha$ absorption at $1577 \AA$ in their FOS data of $\mathrm{H} 1821+643$. In our waveband, we expect to detect C III $\lambda 977$ and Ly $\gamma$ associated with this absorber. Indeed, we detect these strong features at $z=0.29673 \pm 0.00002$. Since both of these features are present, these detections allow us to shift the observations of Savage, Sembach, \& Lu (1995) and Tripp, Lu, \& Savage (1998a,b) to our LSR velocity scale. Based upon these LSR corrected wavelengths, in Table 4 we list all expected intervening absorptions lines from known intervening systems at higher redshift. Table 4 is ordered by strength of the rest-frame ${ }^{6}$ Ly $\alpha$ absorption $\left(\mathcal{W}_{\text {Ly }}\right)$, and breaks naturally into detections and non-detections. Table 4 lists by column: (1) expected wavelength of the absorption based upon the redshift of the system and our LSR correction; (2) line identification; (3) system redshift; (4) rest-frame EW of Ly $\alpha$ in the system; (5) reference for the Ly $\alpha$ detection; (6) whether this feature was detected in our spectrum; and (7) comments, including the observed rest-frame EW.

In addition to the C III $\lambda 977.0$ and Ly $\gamma$ absorptions at $z=0.297$, we detect 6 (possibly 7 ) of the 14 expected features, including $3 \mathrm{Ly} \beta$ and 3 O VI $\lambda 1031.92$ lines. Of these 6 lines, the $1244.6 \AA$ $(\mathrm{Ly} \beta), 1256.5 \AA(\mathrm{Ly} \beta)$, and $1264.1 \AA(\mathrm{O}$ VI $\lambda 1031.92)$ detections were reported by Tripp, Lu, \& Savage (1998a,b) or Savage, Sembach, \& Lu (1995). The $1264 \AA$ O VI $\lambda 1031.92$ absorption system at $z=0.225$ is studied in detail by Savage, Tripp, \& Lu (1998), Barlow \& Tytler (1998), and Tripp, $\mathrm{Lu}, \&$ Savage $(1998 \mathrm{a}, \mathrm{b})$. Our observations suggest that the $z=0.225$ system is actually composed of two components separated by $\sim 75 \mathrm{~km} \mathrm{~s}^{-1}$. This has recently been confirmed by Ly $\delta$ and Ly $\epsilon$ FUSE observations (Shull et al. 2000; Oegerle et al. 2000). We report the first detection of O VI $\lambda 1031.92$ $(1252.2 \AA)$ associated with the detected $\operatorname{Ly} \beta(1244.6 \AA)$ at $z=0.213$. The unambiguous identification of O VI $\lambda 1037.62$ associated with this system is not possible due to HVC S II $\lambda 1259.5$ absorption. However, based upon the strength of the O VI $\lambda 1031.92$ detection $(51 \mathrm{~m} \AA$ ), we would expect a $\sim 34 \mathrm{~m} \AA$ detection of O VI $\lambda 1037.62$. The S II $\lambda 1259.5$ HVC detection $(80 \pm 21 \mathrm{~m} \AA)$ is significantly stronger than our S II $\lambda 1250.6$ and S II $\lambda 1253.8$ HVC detections ( $30 \pm 17$ and $33 \pm 13 \mathrm{~m} \AA$ ) indicating that we are probably detecting O VI $\lambda 1037.62$ at $z=0.213$. In addition, we report the detection of Ly $\beta(1257.7 \AA)$ and O VI $\lambda 1031.92(1257.7 \AA)$ associated with the $z=0.226$ Ly $\alpha$ detection of Tripp, $\mathrm{Lu}$, \& Savage (1998a). As summarized in Table 4, we detect all anticipated absorption features (Ly $\beta$, O VI $\lambda \lambda 1032,1038$, or C III $\lambda$ 977) whose associated Ly $\alpha$ absorption has $\mathcal{W}>163 \mathrm{~m} \AA$.

It is important to note that, due to the uncertainties associated with the redshift determinations of the FOS Ly $\alpha$ detections and the limited resolution of the GHRS/G160M, the possibility of feature misidentifications in this sightline is greater than in the rest of our sample. As a followup study of this target with HST/STIS, we were awarded Cycle 8 observing time to measure the C IV $\lambda \lambda 1548$, 1550 absorption features associated with our O VI $\lambda 1031.9$ detections. We detect $\mathrm{C}$ IV for the strongest systems surveyed and will present these results elsewhere. At the extreme blue end of our spectrum, a feature which is possibly C III $\lambda 977$ associated with the $z=0.261$ system, is marginally detected $(2 \sigma)$. Due to the systematically higher noise level at the edges of the GHRS spectrum,

\footnotetext{
${ }^{6}$ Table 6 of Tripp, Lu, \& Savage (1998a) incorrectly identifies rest-frame values as observed-frame.
} 
this feature is not included by our $S L \geq 3 \sigma$ criteria.

\subsection{IZW1}

The GHRS/G160M spectrum of IZW1 was first reported in Stocke et al. (1995). Except for $\mathrm{Q} 1230+0115$, this is the lowest $\mathrm{S} / \mathrm{N}$ spectrum in our sample $(\sim 13)$, with an average $4 \sigma$ detectable limit of $\mathcal{W}=100 \mathrm{~m} \AA$. We detect no intergalactic Ly $\alpha$ absorbers, although results previously pre-

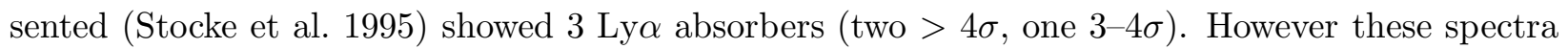
relied on the default 1995 IRAF/STSDAS/CALHRS calibration, which did not correctly handle photocathode blemishes and dead diodes. In both HST exposures, z1a60404n and z2ia0204n, approximately $1 \%$ of the spectrum was affected by a medium photocathode blemish, and $4 \%$ suffered from bad diodes. The previously reported $1222.21 \AA$ absorption feature, now present at only $\mathrm{SL}=1.6 \sigma$, is close to the edge of our spectrum in a region of poor $\mathrm{S} / \mathrm{N}$. The previously reported $1236.5 \AA$ feature is marginally present at $\mathrm{SL}=1.8 \sigma$. This is unfortunate, since two studies (Grogin \& Geller 1998; Stocke et al. 1995) used these results for detailed analysis of the galaxy density distribution along this sightline. Our recalibrations show the previously reported Ly $\alpha$ features to be non-existent or below the $3 \sigma$ level. The only features with $S L \geq 3 \sigma$ in the GHRS/G160M spectra of IZW1 are the Galactic S II features. As previously reported in Stocke et al. (1995), and observed with IUE, the continuum hump at $\sim 1245 \AA$ is rest-frame C III $\lambda 1175$ emission at the redshift of IZW1. The LSR offset for this spectrum was $0.139 \AA$ or $33.4 \mathrm{~km} \mathrm{~s}^{-1}$ at $1253 \AA$.

\subsection{Markarian 279}

This spectrum is dominated by strong Ly $\alpha$ emission from Markarian 279 centered at $1252 \AA$ $(z=0.0294)$. The sightline passes through the HVC complex C, which we detect in S II $\lambda 1250$ at $-160 \mathrm{~km} \mathrm{~s}^{-1}(4.0 \sigma)$ and in $\mathrm{N} \mathrm{V} \lambda 1238$ at $-136 \mathrm{~km} \mathrm{~s}^{-1}(4.4 \sigma)$. Detection of this HVC is not possible in S II $\lambda 1253$ owing to strong intrinsic Ly $\alpha$ absorption at 1253.0-1253.7 $\AA$. While Galactic N V $\lambda 1238$ is strong in this sightline $(11 \sigma, 51 \mathrm{~m} \AA)$, Galactic N V $\lambda 1242$ falls below our $3 \sigma$ threshold for being included in our line list for this object. This alerts us to the possibility that the Galactic N V $\lambda 1238$ absorption may be superimposed on an intergalactic Ly $\alpha$. Although we have only $\sim 23 \AA$ of coverage after applying our $\mathrm{c}_{\mathrm{em}^{-}}-1,200 \mathrm{~km} \mathrm{~s}^{-1}$ blueward limit, we detect six $S L \geq 4 \sigma$ and two $3 \sigma \leq S L<4 \sigma$ Ly $\alpha$ absorbers. The $1241.8 \AA$ Ly $\alpha$ absorption feature could possibly be Si III $\lambda 1206.5$ intrinsic to Mrk 279 at $V_{\mathrm{lsr}} \cong-40 \mathrm{~km} \mathrm{~s}^{-1}$. However, since we do not detect any intrinsic Ly $\alpha$ absorption at this velocity, this is likely to be an intergalactic Ly $\alpha$ absorption and we identify it as such. The LSR offset for this spectrum was $0.116 \AA$ or $27.7 \mathrm{~km} \mathrm{~s}^{-1}$ at $1253 \AA$. 
Table 4: Anticipated Absorption Features in the H1821+643 Spectrum

Detections

\begin{tabular}{|c|c|c|c|c|c|c|}
\hline $\begin{array}{l}\lambda_{\exp } \\
(\AA)\end{array}$ & Identification & $\bar{z}$ & $\begin{array}{c}\mathcal{W}_{\text {Lyo }} \\
(\mathrm{mA})\end{array}$ & Ref & $\overline{\text { Detected }}$ & Comments \\
\hline 1256.53 & $\operatorname{Ly} \beta$ & 0.22489 & $739 \pm 22$ & 1 & Yes & $\mathcal{W}=509 \pm 28 \mathrm{~m} \AA$, two components \\
\hline 1264.13 & OVI 1031.92 & 0.22489 & $739 \pm 22$ & 1 & Yes & $\mathcal{W}=190 \pm 18 \mathrm{~m} \AA, \Delta v \sim 75 \mathrm{~km} \mathrm{~s}^{-1}$ \\
\hline 1261.25 & $\mathrm{Ly} \gamma$ & 0.29673 & $524 \pm 8$ & 1 & Yes & $\mathcal{W}=253 \pm 19 \mathrm{~mA}$ \\
\hline 1267.07 & CIII 977.03 & 0.29673 & $524 \pm 8$ & 1 & Yes & $\mathcal{W}=259 \pm 17 \mathrm{~mA}$ \\
\hline 1244.60 & $\overline{\mathrm{Ly} \beta}$ & 0.21326 & $483 \pm 18$ & 1 & Yes & $\mathcal{W}=153 \pm 18 \mathrm{~m} \AA$ \\
\hline 1252.12 & OVI 1031.92 & 0.21326 & $483 \pm 18$ & 1 & Yes & $\mathcal{W}=51 \pm 17 \mathrm{~mA}$, blended with Ly $\alpha$ at $1252.5 \AA$ \\
\hline 1259.03 & O VI $\lambda 1037.62$ & 0.21326 & $483 \pm 18$ & 1 & Probable & $\mathcal{W} \sim 40 \mathrm{~mA}$, blended with HVC S II $\lambda 1259.5$ \\
\hline 1257.75 & $\operatorname{Ly} \beta$ & 0.22621 & $280 \pm 20$ & 2 & Yes & $\mathcal{W}=20 \pm 14 \mathrm{~mA}$ \\
\hline 1265.36 & OVI 1031.92 & 0.22621 & $280 \pm 20$ & 2 & Yes & $\mathcal{W}=16 \pm 12 \mathrm{~m} \AA$ \\
\hline \multicolumn{7}{|c|}{ Non-Detections } \\
\hline $\begin{array}{l}\lambda_{\exp } \\
(\AA)\end{array}$ & Identification & $\bar{z}$ & 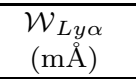 & Ref & $\overline{\text { Detected }}$ & Comments \\
\hline 1237.63 & CIII 977.03 & 0.26660 & $163 \pm 13$ & 1 & No & \\
\hline 1247.04 & $\operatorname{Ly} \beta$ & 0.21577 & $146 \pm 15$ & 2 & No & $<2 \sigma$ \\
\hline 1254.58 & OVI 1031.92 & 0.21577 & $146 \pm 15$ & 2 & Possible & blended with Ly $\alpha$ \\
\hline 1242.93 & $\operatorname{Ly} \beta$ & 0.21176 & $104 \pm 14$ & 2 & No & Blended with N V $\lambda 1242$ \\
\hline 1250.45 & OVI 1031.92 & 0.21176 & $104 \pm 14$ & 2 & Indeterminate & If present, blended with Galactic S II $\lambda 1250.6$ \\
\hline 1259.53 & $\operatorname{Ly} \beta$ & 0.22782 & $71 \pm 11$ & 1 & Indeterminate & If present, blended with Galactic S II $\lambda 1259.5$ \\
\hline 1236.32 & OVI 1031.92 & 0.19794 & $58 \pm 15$ & 1 & No & \\
\hline
\end{tabular}

Note. - All equivalent widths are rest-frame values. These spectral features are anticipated based upon the detection of Ly $\alpha$ absorption at a redshift that places strong non-Ly $\alpha$ lines (e.g., Ly $\beta$ ) in our waveband. The table is ordered by strength of the Ly $\alpha$ absorption and breaks naturally into detections and non-detections.

References. - 1: (GHRS+G160M) Savage, Sembach, \& Lu (1995) or 2: (GHRS+G140L) Tripp, Lu, \& Savage (1998a,b). 


\subsection{Markarian 290}

The Markarian 290 spectrum was obtained by Wakker in 1997 for the purpose of studying the Galactic HVC complex C. This spectrum contains 3 definite and 3 possible $(3 \sigma \leq S L<4 \sigma)$ Ly $\alpha$ absorption features. Our spectrum covers a spectral range of $1232-1263 \AA$ and is dominated by Ly $\alpha$ emission from Mrk 290 at $\sim 1253 \AA$. Along this sightline we detect strong Galactic S II $\lambda \lambda 1250$, 1253, 1259, and Si II $\lambda 1260.4+$ Fe II $\lambda 1260.5$. As determined from Wakker \& van Woerden (1991), the dominant H I-HVC velocity in this direction is $-120 \mathrm{~km} \mathrm{~s}^{-1}$. Wakker et al. (1996) report detections of high velocity gas $(\mathrm{H} \mathrm{I} 21 \mathrm{~cm})$ at $V_{\mathrm{lsr}} \cong-138 \mathrm{~km} \mathrm{~s}^{-1}$ and $-115 \mathrm{~km} \mathrm{~s}^{-1}$. We do not have the spectral resolution to resolve these two HVCs. We detect this combined HVC in Si II $\lambda 1260.4+$ Fe II $\lambda 1260.5$, S II $\lambda \lambda 1250,1253,1259$, and N V $\lambda \lambda 1238,1242$. The S II and Si II+Fe II detections are all heavily blended with intrinsic Ly $\alpha$ absorption, and their EWs are not well determined. The LSR and HVC N V $\lambda \lambda 1238,1242$ detections all fall below the $3 \sigma$ level and are not included in the line list. The HVC detection of $\mathrm{N} \mathrm{V} \lambda 1242.8$ appears to be partially blended with Si III $\lambda 1206.5$ intrinsic to Mrk 290 (which is detected at $\mathrm{SL}=3.7 \sigma$ ). The LSR offset for this spectrum was $0.116 \AA$ or $27.9 \mathrm{~km} \mathrm{~s}^{-1}$ at $1253 \AA$.

While several absorbers are reported in the wavelength range 1245-1248 $\AA$, the rapidly rising continuum on the blue wing of the target's Ly $\alpha$ emission calls their reality into question. Due to our concerns about the reality of these features, we have refit the continuum for Mrk 290 by altering the order of the global fit significantly. The statistical significances of these features remains robust despite these changes, so we list them as definite or possible Ly $\alpha$ features as shown in Figure 12.

\subsection{Markarian 335}

This sightline was previously reported in Stocke et al. (1995). It contains 4 definite Ly $\alpha$ absorption features along with strong Ly $\alpha$ emission from Markarian 335 and Galactic features. Due to calibration improvements, the wavelengths and feature significances have changed slightly from our previous results. Although not listed in Table 9, we also report possible weak detections of Galactic Mg II $\lambda \lambda 1239.9,1240.4(2.5 \sigma$ and $2.7 \sigma)$. The Galactic Mg II $\lambda \lambda 1239.9,1240.4$ absorption occurs at a wavelength at which $\operatorname{Ly} \alpha$ would occur if it were associated with the galaxy identified as $00036+1928$ in the CfA redshift survey and in Table 10 . This galaxy is only $\sim 700 h_{70}^{-1} \mathrm{kpc}$ from the sightline and appears as the small 'v' at $\sim 6,000 \mathrm{~km} \mathrm{~s}^{-1}$ in the Mrk 335 "pie diagrams" of Figure 29. All Ly $\alpha$ absorption features are well-modeled by a single Gaussian component, except for the $1232.9 \AA$ absorption feature, which at higher resolution may actually be two features separated by $\sim 45 \mathrm{~km} \mathrm{~s}^{-1}$. But, at the resolution and $\mathrm{S} / \mathrm{N}$ of our spectrum, it must be considered as a single absorber. Although this sightline passes through Galactic Radio Loop II, no high-velocity Galactic absorptions are detected. The LSR offset for this spectrum was $0.119 \AA$ or $28.6 \mathrm{~km} \mathrm{~s}^{-1}$ at $1253 \AA$. 


\subsection{Markarian 421}

This sightline was previously reported in Shull, Stocke, \& Penton (1996). The Markarian 421 observation (z2ia0104t) suffered from HST target acquisition anomalies, which resulted in 11 of 42 spectral subexposures being removed from the coaddition. These anomalies were not detected by the default calibration used in our previous report, and as a result there are differences in our detections. Most noticeable is the reduced SL of the "mystery absorber" noted in Shull, Stocke, \& Penton (1996) at $1257.1 \AA$, whose velocity was $1150 \mathrm{~km} \mathrm{~s}^{-1}$ higher than that reported for Mrk 421 itself, if it were Ly $\alpha$. This feature, and its companion at $1256.9 \AA$, are now identified as intrinsic Ly $\alpha$, but with $\mathrm{SL}<4 \sigma$. Table 9 indicates our new results, which indicate one definite Ly $\alpha$ absorption feature at $1228.0 \AA$ A. This "void" absorber was first reported in Shull, Stocke, \& Penton (1996).

Although this sightline does not pass through any known Galactic radio loops, it passes between H I-HVC components of the M complex (MI and MII) as discussed in Tufte et al. (1998). Lockman \& Savage (1995) report an H I-HVC at $V_{\mathrm{lsr}}=-58 \mathrm{~km} \mathrm{~s}^{-1}$ with $N_{\mathrm{HI}}=5.9 \times 10^{19} \mathrm{~cm}^{-2}$ towards Mrk 421. We detect a HVC in both S II $\lambda 1250\left(V_{\mathrm{lsr}}=-52 \mathrm{~km} \mathrm{~s}^{-1}\right)$ and S II $\lambda 1253\left(V_{\mathrm{lsr}}=\right.$ $-53 \mathrm{~km} \mathrm{~s}^{-1}$ ). The rest EWs for these S II-HVC detections are $28 \mathrm{~m} \AA$ and $33 \mathrm{~m} \AA$, respectively. If $b=25 \mathrm{~km} \mathrm{~s}^{-1}$, then $N_{\mathrm{SII}} \sim 3.4 \times 10^{14} \mathrm{~cm}^{-2}$ and $N_{\mathrm{SII}} / N_{\mathrm{HI}}=6 \times 10^{-6}$, or $\sim 30 \%$ of the solar abundance of $\mathrm{S} / \mathrm{H}$. Although it is possible that the S II HVC detections could be intrinsic Ly $\alpha$ absorbers, we consider this unlikely due to the presence of absorptions in both components of the S II lines detectable in the observed band.

Also of note is the possibility that the $3 \sigma \mathrm{N} \mathrm{V} \lambda 1238$ detection has a velocity that is more consistent with the detected HVC component. There is an unreported $1.9 \sigma$ detection at the expected LSR position of $\mathrm{N} \mathrm{V} \lambda 1242$. The LSR offset for this spectrum was $0.160 \AA$ or $38.2 \mathrm{~km} \mathrm{~s}^{-1}$ at $1253 \AA$.

\subsection{Markarian 501}

The Markarian 501 sightline was previously reported in Stocke et al. (1995, S95) and passes through the Galactic HVC complex C. Along this sightline, the dominant H I-HVC velocity is at $V_{\mathrm{lsr}} \cong-115 \mathrm{~km} \mathrm{~s}^{-1}$ (Wakker \& van Woerden 1991). However, this is one of our lower $\mathrm{S} / \mathrm{N}$ spectra and we do not detect any HVC absorption. We do detect strong Galactic S II $\lambda \lambda 1250$, 1253 absorption. Due to improvements in calibration, as previously described, our results have changed slightly since S95. Similar to S95, we report 4 definite Ly $\alpha$ absorptions and no possible $(3 \sigma \leq S L<4 \sigma)$ detections. Absorber "A" of S95 is essentially unchanged, but absorbers "B" and "C" of S95 have changed somewhat. Previously, the "B" absorber (1239.97 $\AA$ ) was quoted as having a significance of $3.4 \sigma$, while the "C" absorber $(1246.18 \AA$ ) was quoted as $4.6 \sigma$. Our new results place the S95 "B" absorber's significance at $4.2 \sigma$ and the "C" absorber's at $4.0 \sigma$. Therefore, we now believe the "B" (1239.97 $\AA$ ) feature to be definite, however it may be partially contaminated by 
Galactic Mg II $\lambda 1239.9$ absorption. No Galactic Mg II $\lambda 1240.4$ absorption is detected at the $80 \mathrm{~m} \AA$ $(4 \sigma)$ level. Hence, we cannot estimate the amount of Mg II $\lambda 1239.9$ contamination of the $1239.97 \AA$ feature, if any. We note that, while absorber "C" was identified in S95 as the best example of a Ly $\alpha$ cloud in a void, absorber "B" is located much farther into the void in this region. Therefore, the important conclusions of S95 are unaffected. Calibration improvements have also allowed us to resolve the previously unreported $1251.15 \AA$ Ly $\alpha$ feature from Galactic S II $\lambda 1250.6$ absorption. We have identified this absorption as $\operatorname{Ly} \alpha$ because there is no $\mathrm{H}$ I emission seen at positive velocities in this direction. However, Mrk 501 lies behind complex $\mathrm{C}$ which has blueshifted H I only (Wakker \& van Woerden 1991) which may account for the blueshifted component of S II $\lambda 1253.8$. The LSR offset for this spectrum was $0.232 \AA$ or $55.4 \mathrm{~km} \mathrm{~s}^{-1}$ at $1253 \AA$.

\subsection{Markarian 509}

The Markarian 509 (1231.7 $\AA 1268.8 \AA)$ sightline has been previously reported in Savage, Sembach, \& Lu (1997) and Sembach et al. (1995, 1999, who focused on the Galactic HVCs). These reports were based solely upon the pre-COSTAR HST/G160M observation z1790208m. We detect one definite intergalactic Ly $\alpha$ along this sightline. Our analysis combines this observation with a post-COSTAR observation at a slightly lower wavelength range (1219.5 $\AA$ - 1255.6 $\AA$, see Table 1). The flux level of our HST exposure, z3e70704t, was scaled to be consistent with the initial Mrk 509 exposure. The scaling was applied linearly across the overlap region between exposures. The large gradient in the $\mathcal{W}$ detection limit shortward of $1233 \AA$ is because this portion of the spectrum was observed only with the post-COSTAR exposure. Along this sightline, Sembach et al. $(1995,1999)$ report C IV-HVCs at $-228 \mathrm{~km} \mathrm{~s}^{-1}$ and $-283 \mathrm{~km} \mathrm{~s}^{-1}$, while Anglo-Australian telescope (York 1982) observations of $\mathrm{Na}$ I D lines and $\mathrm{Ca}$ II $\mathrm{H}$ and $\mathrm{K}$ lines indicate Galactic velocity components near $V_{\mathrm{lsr}} \approx+5 \mathrm{~km} \mathrm{~s}^{-1}$ and $+60 \mathrm{~km} \mathrm{~s}^{-1}$. With our GHRS/G160M data we detect HVC gas in S II $\lambda \lambda 1250$, 1253, N V $\lambda 1238, \mathrm{Mg}$ II $\lambda 1239$, and possibly in N V $\lambda 1242$ and Mg II $\lambda 1240$. The $\mathrm{N} \mathrm{V}$ and Mg II Galactic lines show velocity structure over the $V_{\text {lsr }}$ range of -65 to $+60 \mathrm{~km} \mathrm{~s}^{-1}$. We do not have the spectral resolution to confirm the $+5 \mathrm{~km} \mathrm{~s}^{-1}$ component. We do not detect S II $\lambda \lambda 1250,1253$ absorption at $V_{\mathrm{lsr}}=-228$ or $-283 \mathrm{~km} \mathrm{~s}^{-1}$. Intrinsic Ly $\alpha$ associated with Mrk 509 prevents us from detecting HVC absorption due to S II $\lambda 1259$ at these velocities $\left(V_{\mathrm{lsr}}=-228\right.$ and $\left.-283 \mathrm{~km} \mathrm{~s}^{-1}\right)$. Similarly, HVC detection of Si II $\lambda 1260.4+$ Fe II $\lambda 1260.5$ at these velocities is not possible due to Galactic S II $\lambda 1259$, and $\mathrm{Mg}$ II $\lambda \lambda 1239.9,1240.4$ detections are complicated by the presence of Galactic and HVC N V $\lambda \lambda 1238,1242$. We also detect Galactic absorption in N V $\lambda \lambda 1238,1242$, Mg II $\lambda 1239.9$, S II $\lambda \lambda 1250,1253,1259$, and Si II $\lambda 1260.4+$ Fe II $\lambda 1260.5$, and possibly C I $\lambda 1260$ (heavily blended with Si II $\lambda 1260.4+$ Fe II $\lambda 1260.5$, and Si II $\lambda 1260.4+$ Fe II $\lambda 1260.5$ HVC, and a possible intrinsic Ly $\alpha$ absorption in Mrk 509). We detect high-velocity Galactic gas at +60 and $-65 \mathrm{~km} \mathrm{~s}^{-1}$ in N V $\lambda 1238$, S II $\lambda \lambda 1250,1253,1259$ and possibly Si II $\lambda 1260.4+$ Fe II $\lambda 1260.5$. The Si II $\lambda 1260.4+$ Fe II $\lambda 1260.5$ absorption is apparently heavily blended with H I absorption intrinsic to Mrk 509, and possible Galactic C I $\lambda 1260$. Along with these HVCs, the spectrum of Mrk 509 contains a single definite $(S L \geq 4 \sigma)$ Ly $\alpha$ absorption feature at $2560 \mathrm{~km} \mathrm{~s}^{-1}$. The LSR offset for 
this spectrum was $0.060 \AA$ or $14.4 \mathrm{~km} \mathrm{~s}^{-1}$ at $1253 \AA$.

Numerous strong intrinsic Ly $\alpha$ absorption lines are observed in our Mrk 509 spectrum. This absorption shows significant structure over the velocity range of -450 to $+200 \mathrm{~km} \mathrm{~s}^{-1}$. Our modelling suggests 7-9 separate velocity components. Recent FUSE observations (Kriss et al. 2000) detect these absorbers in $\mathrm{Ly} \beta, \mathrm{C}$ III $\lambda 977.0$, and O VI $\lambda 1031.9$, which they model with 7 separate components. Efforts are underway to match our Ly $\alpha$ detections with the FUSE detections to determine the metallicity and $b$-values of these intrinsic absorbers. These results will be reported elsewhere (Shull et al. 2000).

\subsection{Markarian 817}

The sightline towards Markarian 817 passes through HVC complex C. The dominant H I-HVC velocity in this direction is $-115 \mathrm{~km} \mathrm{~s}^{-1}$ (Wakker \& van Woerden 1991). We detect strong Galactic S II $\lambda \lambda 1250,1253$, two HVCs $\left(V_{\mathrm{lsr}} \approx-45 \mathrm{~km} \mathrm{~s}^{-1}\right.$ and $\left.-110 \mathrm{~km} \mathrm{~s}^{-1}\right)$ in S II $\lambda \lambda 1250,1253$ as well as N V $\lambda \lambda 1238,1242$ (Galactic+HVCs). The N V $\lambda 1238.8$ and N V $\lambda 1242.8$ regions are both confused with significant structure. The N V $\lambda 1238.8$ result is the combination of LSR + HVC absorptions, while the $1242.5 \AA$ detection appears to be associated with the $\mathrm{HVC}$ at $V_{\mathrm{lsr}}=-45 \mathrm{~km} \mathrm{~s}^{-1}$. The $V_{\mathrm{lsr}}=-110 \mathrm{~km} \mathrm{~s}^{-1} \mathrm{HVC}$ is also detected in $\mathrm{N} \mathrm{V} \lambda 1242.8$ at the $2.8 \sigma$ level. Galactic $\mathrm{N} \mathrm{V} \lambda 1242.8$ at LSR is also detected, but at the anomalously low $3.1 \sigma$ level. We do not detect any Galactic Mg II $\lambda \lambda 1239.9,1240.4$ absorption, although it is possible that the reported $1239.5 \AA$ feature is contaminated with weak Mg II $\lambda 1239.9$ HVC absorption. Note that, along this sightline the HVC $\mathrm{N} \mathrm{V}$ and $\mathrm{Mg}$ II absorptions are stronger than the LSR Galactic components. In addition to the Galactic detections, this rich sightline contains 9 definite Ly $\alpha$ absorption features and several Ly $\alpha$ absorptions identified as intrinsic to Mrk 817. The LSR offset for this spectrum was $0.083 \AA$ or $19.8 \mathrm{~km} \mathrm{~s}^{-1}$ at $1253 \AA$.

\subsection{PKS 2155-304}

The pre-COSTAR spectrum of PKS 2155-304 contains 8 definite $(S L \geq 4 \sigma)$ and 4 possible $(3 \sigma \leq S L<4 \sigma)$ Ly $\alpha$ absorption features, as well as Galactic Mg II $\lambda \lambda 1239.9,1240.4$ and S II $\lambda \lambda 1250,1253$ absorption. An analysis of this sightline has been previously reported in Shull et al. (1998). Improvements in the calibration process since the publication of that paper produce slightly different results. Most notable is the inclusion of the $1235.8 \AA \mathrm{Ly} \alpha$ feature, which is blended with the $1236.0 \AA$ and $1236.4 \AA$ features. Our restriction of $b<100 \mathrm{~km} \mathrm{~s}^{-1}$, which was not present in our previous reduction, forced us to add a third component to this blend. As a result, EWs for the $1235.6 \AA$ and $1236.4 \AA$ features are slightly different than previously reported. The $1238.7 \AA$ feature is partially blended with Galactic N V $\lambda 1238$. In our previous paper (Shull et al. 1998) we estimated the $\mathrm{N}$ V contribution, while for this paper we have fitted blended components to both 
$\mathrm{N} \mathrm{V} \lambda 1238$ and Ly $\alpha$. The feature marked as possible Ly $\alpha$ at $1256.7 \AA$ could also be a Galactic S II $\lambda 1253$ HVC. Galactic N V $\lambda \lambda 1238,1242$, Mg II $\lambda \lambda 1239.9,1240.4$, and S II $\lambda \lambda 1250,1253$ are all present and strong. The LSR offset for the pre-COSTAR spectrum was $0.013 \AA$ or $3.0 \mathrm{~km} \mathrm{~s}^{-1}$ at $1253 \AA$.

The post-COSTAR spectrum of PKS 2155-304 was also reported in Shull et al. (1998) and contains 8 definite and 1 possible Ly $\alpha$ absorption features. As with the pre-COSTAR spectrum, minor improvements to the calibration process produce slightly improved feature modelings. A detailed discussion of the clump of 8 Ly $\alpha$ absorbers near $c z=17,000 \mathrm{~km} \mathrm{~s}^{-1}$ is given in Shull et al. (1998). The third (most blueward) component of the 1284-1285 $\AA$ Ly $\alpha$ complex is only marginally required by our fitting routines. Relaxing our $b$-value $\leq 100 \mathrm{~km} \mathrm{~s}^{-1}$ criteria provides an adequate 2 component spectral fit as originally reported in Shull et al. (1998). Galactic S II $\lambda 1259$ is present along with strong Si II $\lambda 1260.4+$ Fe II $\lambda 1260.5$, and a Si II $\lambda 1260.4+$ Fe II $\lambda 1260.5$ HVC detection (Sembach et al. 1999). The post-COSTAR PKS 2155-304 spectrum was corrected to the LSR using the Galactic H I and only the S II line (1259 $\AA)$. The LSR offset for the postCOSTAR spectrum was $0.073 \AA$ or $17.4 \mathrm{~km} \mathrm{~s}^{-1}$ at $1253 \AA$. The PKS 2155-304 sightline does not pass through any known H I-HVCs or Galactic radio loops, although C IV-HVCs were detected by Sembach et al. (1995) at $V_{\mathrm{lsr}}=-260$ and $-145 \mathrm{~km} \mathrm{~s}^{-1}$. We detect the $V_{\mathrm{lsr}}=-145 \mathrm{~km} \mathrm{~s}^{-1}$ component in Si II $\lambda 1260.4+$ Fe II $\lambda 1260.5$ (at $V_{\mathrm{lsr}}=-154 \mathrm{~km} \mathrm{~s}^{-1}$ ) and report a possible $(2.4 \sigma)$ detection of the $V_{\mathrm{lsr}}=-260 \mathrm{~km} \mathrm{~s}^{-1}$ component in S II $\lambda 1253$.

\subsection{Q1230+0115}

The spectrum of the QSO Q1230+0115 contains 6 definite and 4 possible $(3 \sigma \leq S L<4 \sigma)$ Ly $\alpha$ absorption features. This sightline does not pass through any known radio loops or HVCs and is only $0.91^{\circ}$ degrees from the 3C 273 sightline. This sightline shows a cluster of four Ly $\alpha$ absorbers within $1 \AA\left(\sim 200 \mathrm{~km} \mathrm{~s}^{-1}\right)$ of $1222.5 \AA\left(1,700 \mathrm{~km} \mathrm{~s}^{-1}\right)$ and an additional Ly $\alpha$ absorber at $1225 \AA$ $\left(2,303 \mathrm{~km} \mathrm{~s}^{-1}\right)$. A possible $(3.1 \sigma)$ Ly $\alpha$ absorber is also present at $1223.9 \AA\left(2037 \mathrm{~km} \mathrm{~s}^{-1}\right)$. Another possible absorber $(2.0 \sigma)$ at $1221.05 \AA$ is not statistically significant due a minor diode problem close to its location (see the EW detection limit panel in Figure 20). The 3C 273 sightline also contains an absorber at $1,586 \mathrm{~km} \mathrm{~s}^{-1}$ and 2 possible absorbers at 2,200-2,300 $\mathrm{km} \mathrm{s}^{-1}$ which appear to closely match detections in this sightline. The transverse separations between these sightlines at $1,500 \mathrm{~km} \mathrm{~s}^{-1}$ and $2,300 \mathrm{~km} \mathrm{~s}^{-1}$ are 340 and $520 h_{70}^{-1} \mathrm{kpc}$, respectively. This sightline pair is investigated further in Paper III. Galactic N V $\lambda 1238$ is detected at the $2.7 \sigma$ level, while N V $\lambda 1242$ is marginally detected at the $1.7 \sigma$ level. The $\mathrm{N} \mathrm{V} \lambda 1242$ absorption is partially blended with the possible absorber at $1242.9 \AA$. The Mg II $\lambda \lambda 1239.9$, 1240.4 lines are not detected in this sightline. This spectrum has the lowest $\mathrm{S} / \mathrm{N}(\sim 9)$ of our sample and is being reobserved in cycle 7 with HST/STIS. The LSR offset for this spectrum was $-0.003 \AA$ or $-0.7 \mathrm{~km} \mathrm{~s}^{-1}$ at $1253 \AA$. 


\section{Master Lists of Detected Absorption Features}

We consider absorption features with significance levels $S L \geq 4 \sigma$ to be definite, while absorption lines with $3 \sigma \leq S L<4 \sigma$ are classified as possible. Table 5 presents all definite and Table 6 all possible intergalactic Ly $\alpha$ absorptions. Table 7 presents a summary of all definite Galactic HVC clouds. Due to the overlap between the regions of the sky covered by the HVC complex $\mathrm{C}$ and the initial CfA slices, we detect Galactic HVCs in 8 of our 15 sightlines. This fraction is considerably larger than the value of $37 \%$ found for HVCs seen in $21 \mathrm{~cm}$ emission toward AGN sightlines down to $N_{\mathrm{HI}}=7 \times 10^{17} \mathrm{~cm}^{-2}$. (Murphy, Lockman, \& Savage 1995). Hence, we expect that the HVC detection rate will decrease with future observations due to this unintentional overlap. Table 8 shows a list of all definite lines determined to be intrinsic to the observed target.

The first column of each of the master tables indicates the name of the target in which the feature was detected. An asterisk before the target name denotes a detection in pre-COSTAR data. The second column indicates the LSR-adjusted wavelength and wavelength uncertainty for each feature. The third column lists the recession velocity (for Ly $\alpha$ features, Tables 5 and 6), velocity relative to LSR (HVC features, Table 7), or velocity relative to the narrow emission line rest frame of the target (intrinsic features, Table 8). All velocities are quoted as $c z$, in units of $\mathrm{km} \mathrm{s}^{-1}$. Velocity uncertainties based upon the total wavelength uncertainties (see $\S 2.3 .3$ ) are also provided. The fourth column provides the single-component rest-frame Doppler width $\left(b\right.$ in $\left.\mathrm{km} \mathrm{s}^{-1}\right)$ and its $1 \sigma$ uncertainty for each feature as estimated from the Gaussian width $\left(\mathbf{W}_{G}=b_{\text {obs }} / \sqrt{2}\right)$ of the fitted feature, restricted to the range of $12<b_{\mathrm{obs}}<100 \mathrm{~km} \mathrm{~s}^{-1}$. The Doppler widths have been corrected for the spectral resolution of the GHRS (FWHM $19 \mathrm{~km} \mathrm{~s}^{-1}$ ) and our pre-fit smoothing (see $\S 2.3 .1$ and eq. 2). As discussed in Paper II, the measured $b$-values in pre-COSTAR data are occasionally artificially large. The fifth column lists the rest-frame $\mathrm{EW}(\mathcal{W}$ in $\mathrm{m} \AA)$ and $1 \sigma$ uncertainty of each absorption feature. The sixth column indicates the significance level (SL in $\sigma$ ) of each feature. In Tables 7 , the seventh column indicates the atomic identification of the detected absorption. In Table 8, the final column gives the redshift of the intrinsic system. 
Table 5. Definite $(S L \geq 4 \sigma)$ Intergalactic Ly $\alpha$ Features

\begin{tabular}{|c|c|c|c|c|c|}
\hline Target & $\begin{array}{c}\text { Wavelength } \\
(\AA)\end{array}$ & $\begin{array}{l}\text { Velocity } \\
\left(\mathrm{km} \mathrm{s}^{-1}\right)\end{array}$ & $\begin{array}{c}b \\
\left(\mathrm{~km} \mathrm{~s}^{-1}\right)\end{array}$ & $\begin{array}{c}\mathcal{W} \\
(\mathrm{m} \AA)\end{array}$ & $\begin{array}{l}\mathrm{SL} \\
(\sigma)\end{array}$ \\
\hline$* 3 \mathrm{C} 273$ & $1219.786 \pm 0.024$ & $1015 \pm 6$ & $69 \pm 5$ & $369 \pm 36$ & 34.8 \\
\hline$* 3 \mathrm{C} 273$ & $1222.100 \pm 0.023$ & $1586 \pm 6$ & $72 \pm 4$ & $373 \pm 30$ & 42.0 \\
\hline$* 3 \mathrm{C} 273$ & $1224.954 \pm 0.029$ & $2290 \pm 7$ & $54 \pm 33$ & $35 \pm 30$ & 4.2 \\
\hline$* 3 \mathrm{C} 273$ & $1247.593 \pm 0.046$ & $7872 \pm 11$ & $34 \pm 17$ & $33 \pm 18$ & 5.8 \\
\hline$* 3 \mathrm{C} 273$ & $1251.485 \pm 0.032$ & $8832 \pm 8$ & $61 \pm 10$ & $114 \pm 25$ & 14.8 \\
\hline$* 3 \mathrm{C} 273$ & $1255.542 \pm 0.069$ & $9833 \pm 17$ & $64 \pm 24$ & $46 \pm 22$ & 5.9 \\
\hline$* 3 \mathrm{C} 273$ & $1275.243 \pm 0.031$ & $14691 \pm 7$ & $61 \pm 8$ & $140 \pm 25$ & 16.3 \\
\hline$* 3 \mathrm{C} 273$ & $1276.442 \pm 0.059$ & $14987 \pm 14$ & $52 \pm 20$ & $46 \pm 22$ & 5.4 \\
\hline$* 3 \mathrm{C} 273$ & $1277.474 \pm 0.136$ & $15241 \pm 33$ & $88 \pm 52$ & $52 \pm 40$ & 6.3 \\
\hline$* 3 \mathrm{C} 273$ & $1280.267 \pm 0.077$ & $15930 \pm 19$ & $71 \pm 28$ & $64 \pm 33$ & 6.4 \\
\hline$* 3 \mathrm{C} 273$ & $1289.767 \pm 0.098$ & $18273 \pm 24$ & $82 \pm 36$ & & 6.1 \\
\hline$* 3 \mathrm{C} 273$ & $1292.851 \pm 0.051$ & $19033 \pm 12$ & $45 \pm 17$ & $47 \pm 22$ & 5.5 \\
\hline$* 3 \mathrm{C} 273$ & $1296.591 \pm 0.025$ & $19956 \pm 6$ & $62 \pm 4$ & $297 \pm 25$ & 33.0 \\
\hline AKN120 & $1232.052 \pm 0.034$ & $4040 \pm 8$ & $32 \pm 11$ & $48 \pm 18$ & 5.5 \\
\hline AKN120 & $1242.972 \pm 0.028$ & $6733 \pm 7$ & $33 \pm 8$ & $53 \pm 13$ & 9.2 \\
\hline AKN120 & $1247.570 \pm 0.087$ & $7867 \pm 21$ & $34 \pm 35$ & $20 \pm 25$ & 4.3 \\
\hline AKN120 & $1247.948 \pm 0.023$ & $7960 \pm 5$ & $27 \pm 4$ & $147 \pm 22$ & 31.4 \\
\hline AKN120 & $1248.192 \pm 0.027$ & & & & 14.1 \\
\hline FAIRALL9 & $1240.988 \pm 0.038$ & $6244 \pm 9$ & $35 \pm 13$ & $22 \pm 9$ & 5.3 \\
\hline FAIRALL9 & $1244.462 \pm 0.034$ & $7100 \pm 8$ & $39 \pm 11$ & $32 \pm 10$ & 8.2 \\
\hline FAIRALL9 & $1254.139 \pm 0.024$ & $9487 \pm 6$ & $43 \pm 6$ & $84 \pm 13$ & 26.5 \\
\hline FAIRALL9 & $1262.864 \pm 0.029$ & $11638 \pm 7$ & $28 \pm 13$ & & 5.2 \\
\hline FAIRALL9 & $1263.998 \pm 0.041$ & $11918 \pm 10$ & $42 \pm 15$ & $22 \pm 9$ & 7.0 \\
\hline FAIRALL9 & $1264.684 \pm 0.073$ & $12087 \pm 18$ & $44 \pm 33$ & $30 \pm 28$ & 10.2 \\
\hline FAIRALL9 & $1265.104 \pm 0.026$ & $12191 \pm 6$ & $23 \pm 7$ & $28 \pm 7$ & 9.5 \\
\hline FAIRALL9 & $1265.970 \pm 0.117$ & $12404 \pm 29$ & $32 \pm 23$ & $19 \pm 23$ & 7.0 \\
\hline $\mathrm{H} 1821+643$ & $1245.440 \pm 0.023$ & $7342 \pm 5$ & $47 \pm 3$ & $298 \pm 20$ & 42.4 \\
\hline $\mathrm{H} 1821+643$ & $1246.301 \pm 0.036$ & $7554 \pm 9$ & $41 \pm 14$ & $50 \pm 24$ & 7.1 \\
\hline $\mathrm{H} 1821+643$ & $1247.583 \pm 0.029$ & $7870 \pm 7$ & $19 \pm 9$ & $40 \pm 17$ & 5.4 \\
\hline H1821+643 & $1247.937 \pm 0.033$ & $7957 \pm 8$ & $33 \pm 10$ & $68 \pm 38$ & 9.4 \\
\hline $\mathrm{H} 1821+643$ & $1265.683 \pm 0.025$ & $12334 \pm 6$ & $28 \pm 6$ & $64 \pm 15$ & 11.8 \\
\hline MARK279 & $1236.942 \pm 0.030$ & $5246 \pm 7$ & $27 \pm 9$ & $30 \pm 10$ & 5.6 \\
\hline
\end{tabular}


Table 5-Continued

\begin{tabular}{lccccc}
\hline \hline Target & Wavelength & Velocity & $b$ & $\mathcal{W}$ & SL \\
& $(\AA)$ & $\left(\mathrm{km} \mathrm{s}^{-1}\right)$ & $\left(\mathrm{km} \mathrm{s}^{-1}\right)$ & $(\mathrm{mA})$ & $(\sigma)$ \\
\hline MARK279 & $1241.509 \pm 0.029$ & $6372 \pm 7$ & $18 \pm 4$ & $58 \pm 7$ & 14.2 \\
MARK279 & $1241.805 \pm 0.023$ & $6445 \pm 6$ & $21 \pm 4$ & $40 \pm 7$ & 10.4 \\
MARK279 & $1243.753 \pm 0.023$ & $6925 \pm 5$ & $26 \pm 3$ & $65 \pm 8$ & 16.8 \\
MARK279 & $1247.216 \pm 0.024$ & $7779 \pm 6$ & $28 \pm 5$ & $48 \pm 9$ & 15.1 \\
MARK279 & $1247.533 \pm 0.042$ & $7858 \pm 10$ & $34 \pm 14$ & $21 \pm 10$ & 6.7 \\
MARK290 & $1234.597 \pm 0.027$ & $4667 \pm 7$ & $26 \pm 8$ & $60 \pm 18$ & 7.2 \\
MARK290 & $1244.408 \pm 0.032$ & $7087 \pm 8$ & $23 \pm 11$ & $23 \pm 10$ & 4.3 \\
MARK290 & $1245.536 \pm 0.025$ & $7365 \pm 6$ & $11 \pm 9$ & $21 \pm 7$ & 4.2 \\
*MARK335 & $1223.637 \pm 0.026$ & $1965 \pm 6$ & $75 \pm 7$ & $229 \pm 30$ & 28.1 \\
*MARK335 & $1224.974 \pm 0.049$ & $2295 \pm 12$ & $73 \pm 17$ & $81 \pm 26$ & 10.2 \\
*MARK335 & $1232.979 \pm 0.057$ & $4268 \pm 14$ & $51 \pm 20$ & $33 \pm 16$ & 5.1 \\
*MARK335 & $1241.093 \pm 0.026$ & $6269 \pm 6$ & $75 \pm 6$ & $130 \pm 14$ & 30.3 \\
MARK421 & $1227.977 \pm 0.025$ & $3035 \pm 6$ & $35 \pm 5$ & $86 \pm 15$ & 12.4 \\
*MARK501 & $1234.572 \pm 0.039$ & $4661 \pm 10$ & $60 \pm 13$ & $161 \pm 43$ & 11.1 \\
*MARK501 & $1239.968 \pm 0.029$ & $5992 \pm 7$ & $59 \pm 39$ & $55 \pm 46$ & 4.2 \\
*MARK501 & $1246.177 \pm 0.069$ & $7523 \pm 17$ & $48 \pm 26$ & $53 \pm 36$ & 4.0 \\
*MARK501 & $1251.152 \pm 0.029$ & $8750 \pm 7$ & $77 \pm 49$ & $66 \pm 57$ & 4.9 \\
MARK509 & $1226.050 \pm 0.025$ & $2560 \pm 6$ & $40 \pm 5$ & $209 \pm 32$ & 14.4 \\
MARK817 & $1223.507 \pm 0.037$ & $1933 \pm 9$ & $34 \pm 13$ & $29 \pm 13$ & 5.3 \\
MARK817 & $1224.172 \pm 0.023$ & $2097 \pm 5$ & $40 \pm 4$ & $135 \pm 15$ & 25.3 \\
MARK817 & $1234.657 \pm 0.041$ & $4682 \pm 10$ & $40 \pm 15$ & $23 \pm 11$ & 5.6 \\
MARK817 & $1236.303 \pm 0.023$ & $5088 \pm 6$ & $84 \pm 4$ & $207 \pm 14$ & 56.3 \\
MARK817 & $1236.902 \pm 0.027$ & $5236 \pm 7$ & $24 \pm 7$ & $25 \pm 7$ & 6.6 \\
MARK817 & $1239.159 \pm 0.029$ & $5793 \pm 7$ & $39 \pm 12$ & $34 \pm 13$ & 8.7 \\
MARK817 & $1241.034 \pm 0.024$ & $6255 \pm 6$ & $29 \pm 5$ & $37 \pm 8$ & 11.6 \\
MARK817 & $1245.395 \pm 0.051$ & $7330 \pm 13$ & $51 \pm 18$ & $17 \pm 7$ & 5.9 \\
MARK817 & $1247.294 \pm 0.044$ & $7799 \pm 11$ & $56 \pm 16$ & $28 \pm 9$ & 10.1 \\
*PKS2155-304 & $1226.345 \pm 0.060$ & $2632 \pm 15$ & $61 \pm 33$ & $42 \pm 40$ & 9.2 \\
*PKS2155-304 & $1226.964 \pm 0.065$ & $2785 \pm 16$ & $64 \pm 26$ & $36 \pm 22$ & 7.9 \\
*PKS2155-304 & $1232.016 \pm 0.049$ & $4031 \pm 12$ & $39 \pm 17$ & $21 \pm 11$ & 4.2 \\
*PKS2155-304 & $1235.748 \pm 0.029$ & $4951 \pm 7$ & $68 \pm 15$ & $64 \pm 23$ & 14.3 \\
*PKS2155-304 & $1235.998 \pm 0.029$ & $5013 \pm 7$ & $58 \pm 11$ & $82 \pm 22$ & 18.1
\end{tabular}


Table 5-Continued

\begin{tabular}{cccccc}
\hline \hline Target & $\begin{array}{c}\text { Wavelength } \\
(\AA)\end{array}$ & $\begin{array}{c}\text { Velocity } \\
\left(\mathrm{km} \mathrm{s}^{-1}\right)\end{array}$ & $\begin{array}{c}b \\
\left(\mathrm{~km} \mathrm{~s}^{-1}\right)\end{array}$ & $\begin{array}{c}\mathcal{W} \\
(\mathrm{m} \AA)\end{array}$ & $\begin{array}{c}\text { SL } \\
(\sigma)\end{array}$ \\
\hline *PKS2155-304 & $1236.426 \pm 0.029$ & $5119 \pm 7$ & $80 \pm 5$ & $218 \pm 20$ & 48.1 \\
$*$ PKS2155-304 & $1238.451 \pm 0.029$ & $5618 \pm 7$ & $33 \pm 14$ & $29 \pm 15$ & 6.7 \\
$*$ PKS2155-304 & $1238.673 \pm 0.031$ & $5673 \pm 8$ & $30 \pm 12$ & $39 \pm 16$ & 9.1 \\
PKS2155-304 & $1270.784 \pm 0.027$ & $13591 \pm 6$ & $39 \pm 6$ & $101 \pm 18$ & 12.5 \\
PKS2155-304 & $1281.375 \pm 0.024$ & $16203 \pm 5$ & $58 \pm 3$ & $346 \pm 23$ & 44.8 \\
PKS2155-304 & $1281.867 \pm 0.061$ & $16325 \pm 15$ & $49 \pm 22$ & $62 \pm 34$ & 8.2 \\
PKS2155-304 & $1284.301 \pm 0.030$ & $16925 \pm 7$ & $19 \pm 13$ & $43 \pm 37$ & 5.7 \\
PKS2155-304 & $1284.497 \pm 0.039$ & $16973 \pm 9$ & $63 \pm 6$ & $389 \pm 68$ & 50.9 \\
PKS2155-304 & $1285.086 \pm 0.038$ & $17119 \pm 9$ & $87 \pm 11$ & $448 \pm 79$ & 57.9 \\
PKS2155-304 & $1287.497 \pm 0.024$ & $17713 \pm 6$ & $35 \pm 5$ & $139 \pm 21$ & 18.4 \\
PKS2155-304 & $1288.958 \pm 0.029$ & $18073 \pm 7$ & $47 \pm 8$ & $99 \pm 20$ & 13.2 \\
Q1230+0115 & $1221.711 \pm 0.026$ & $1490 \pm 6$ & $21 \pm 8$ & $138 \pm 42$ & 6.3 \\
Q1230+0115 & $1222.425 \pm 0.035$ & $1666 \pm 9$ & $54 \pm 10$ & $385 \pm 94$ & 16.9 \\
Q1230+0115 & $1222.747 \pm 0.029$ & $1745 \pm 7$ & $40 \pm 12$ & $241 \pm 99$ & 10.9 \\
Q1230+0115 & $1223.211 \pm 0.051$ & $1860 \pm 13$ & $48 \pm 21$ & $142 \pm 81$ & 6.6 \\
Q1230+0115 & $1225.000 \pm 0.024$ & $2301 \pm 6$ & $55 \pm 6$ & $439 \pm 57$ & 23.9 \\
Q1230+0115 & $1253.145 \pm 0.031$ & $9242 \pm 8$ & $72 \pm 8$ & $301 \pm 49$ & 24.8 \\
\hline
\end{tabular}


Table 6. Possible $(3 \sigma \leq S L<4 \sigma)$ Intergalactic Ly $\alpha$ features

\begin{tabular}{lcrccc}
\hline \hline \multicolumn{1}{c}{ Target } & Wavelength & $\begin{array}{c}\text { Velocity } \\
\left(\mathrm{km} \mathrm{s}^{-1}\right)\end{array}$ & $\begin{array}{c}b \\
\left(\mathrm{~km} \mathrm{~s}^{-1}\right)\end{array}$ & $\begin{array}{c}\mathcal{W} \\
(\mathrm{mA})\end{array}$ & $\begin{array}{c}\mathrm{SL} \\
(\sigma)\end{array}$ \\
\hline *3C273 & $1224.587 \pm 0.150$ & $2199 \pm 37$ & $57 \pm 55$ & $29 \pm 35$ & 3.5 \\
*3C273 & $1234.704 \pm 0.029$ & $4694 \pm 7$ & $69 \pm 61$ & $25 \pm 29$ & 3.5 \\
*3C273 & $1265.701 \pm 0.064$ & $12338 \pm 16$ & $33 \pm 26$ & $21 \pm 18$ & 3.3 \\
*3C273 & $1266.724 \pm 0.084$ & $12590 \pm 21$ & $52 \pm 30$ & $24 \pm 18$ & 3.6 \\
*3C273 & $1268.969 \pm 0.076$ & $13144 \pm 19$ & $43 \pm 28$ & $18 \pm 15$ & 3.1 \\
AKN120 & $1223.088 \pm 0.039$ & $1829 \pm 10$ & $25 \pm 17$ & $64 \pm 48$ & 3.7 \\
AKN120 & $1247.267 \pm 0.104$ & $7792 \pm 26$ & $34 \pm 32$ & $19 \pm 22$ & 3.9 \\
ESO141-G55 & $1249.932 \pm 0.036$ & $8449 \pm 9$ & $17 \pm 15$ & $12 \pm 8$ & 3.0 \\
ESO141-G55 & $1252.483 \pm 0.041$ & $9078 \pm 10$ & $23 \pm 16$ & $12 \pm 7$ & 3.1 \\
FAIRALL9 & $1265.407 \pm 0.029$ & $12265 \pm 7$ & $19 \pm 18$ & $11 \pm 8$ & 3.8 \\
H1821+643 & $1238.014 \pm 0.036$ & $5510 \pm 9$ & $21 \pm 14$ & $23 \pm 13$ & 3.3 \\
H1821+643 & $1240.569 \pm 0.036$ & $6140 \pm 9$ & $14 \pm 16$ & $24 \pm 16$ & 3.4 \\
H1821+643 & $1244.966 \pm 0.031$ & $7225 \pm 8$ & $15 \pm 12$ & $25 \pm 13$ & 3.5 \\
H1821+643 & $1247.362 \pm 0.029$ & $7815 \pm 7$ & $36 \pm 34$ & $27 \pm 29$ & 3.7 \\
H1821+643 & $1252.477 \pm 0.042$ & $9077 \pm 10$ & $21 \pm 15$ & $23 \pm 15$ & 3.6 \\
H1821+643 & $1254.874 \pm 0.099$ & $9668 \pm 24$ & $24 \pm 31$ & $21 \pm 25$ & 3.6 \\
MARK279 & $1237.915 \pm 0.029$ & $5486 \pm 7$ & $30 \pm 29$ & $17 \pm 18$ & 3.5 \\
MARK279 & $1238.502 \pm 0.047$ & $5631 \pm 12$ & $21 \pm 23$ & $18 \pm 18$ & 3.7 \\
MARK290 & $1232.797 \pm 0.064$ & $4224 \pm 16$ & $21 \pm 29$ & $41 \pm 49$ & 3.6 \\
MARK290 & $1235.764 \pm 0.044$ & $4955 \pm 11$ & $26 \pm 18$ & $28 \pm 19$ & 3.4 \\
MARK290 & $1245.869 \pm 0.026$ & $7447 \pm 6$ & $8 \pm 12$ & $18 \pm 7$ & 3.6 \\
$*$ PKS2155-304 & $1234.767 \pm 0.051$ & $4709 \pm 12$ & $32 \pm 24$ & $15 \pm 14$ & 3.3 \\
$*$ PKS2155-304 & $1246.990 \pm 0.029$ & $7724 \pm 7$ & $31 \pm 35$ & $13 \pm 16$ & 3.0 \\
$*$ PKS2155-304 & $1247.510 \pm 0.029$ & $7852 \pm 7$ & $30 \pm 31$ & $13 \pm 16$ & 3.1 \\
$*$ PKS2155-304 & $1255.084 \pm 0.041$ & $9720 \pm 10$ & $24 \pm 16$ & $13 \pm 9$ & 3.0 \\
$*$ PKS2155-304 & $1256.636 \pm 0.042$ & $10102 \pm 10$ & $25 \pm 15$ & $14 \pm 8$ & 3.3 \\
PKS2155-304 & $1264.806 \pm 0.058$ & $12117 \pm 14$ & $39 \pm 20$ & $31 \pm 19$ & 3.6 \\
Q1230+0115 & $1236.045 \pm 0.041$ & $5025 \pm 10$ & $24 \pm 15$ & $53 \pm 31$ & 3.1 \\
Q1230+0115 & $1242.897 \pm 0.044$ & $6714 \pm 11$ & $19 \pm 19$ & $45 \pm 35$ & 3.2 \\
Q1230+0115 & $1246.254 \pm 0.049$ & $7542 \pm 12$ & $31 \pm 18$ & $44 \pm 27$ & 3.3 \\
\hline
\end{tabular}


Table 7. Galactic HVC features with $S L \geq 4 \sigma$.

\begin{tabular}{|c|c|c|c|c|c|c|}
\hline Target & $\begin{array}{l}\text { Wavelength } \\
(\AA)\end{array}$ & $\begin{array}{l}\text { Velocity } \\
\left(\mathrm{km} \mathrm{s}^{-1}\right)\end{array}$ & $\begin{array}{c}b \\
\left(\mathrm{~km} \mathrm{~s}^{-1}\right)\end{array}$ & $\begin{array}{c}\mathcal{W} \\
(\mathrm{m} \AA)\end{array}$ & $\begin{array}{l}\mathrm{SL} \\
(\sigma)\end{array}$ & ID \\
\hline ESO141-G55 & $1253.444 \pm 0.038$ & $-85 \pm 9$ & $27 \pm 13$ & $18 \pm 9$ & 4.7 & SII1253.8 \\
\hline ESO141-G55 & $1259.304 \pm 0.029$ & $-47 \pm 7$ & $48 \pm 23$ & $23 \pm 14$ & 5.8 & SII1259.5 \\
\hline ESO141-G55 & $1260.204 \pm 0.029$ & $-70 \pm 7$ & $44 \pm 6$ & $83 \pm 73$ & 21.1 & $\mathrm{SiII}+\mathrm{FeII} 1260.5$ \\
\hline FAIRALL9 & $1251.367 \pm 0.025$ & $188 \pm 6$ & $32 \pm 5$ & $41 \pm 7$ & 11.3 & SII1250.6 \\
\hline FAIRALL9 & $1254.572 \pm 0.023$ & $182 \pm 5$ & $27 \pm 4$ & $56 \pm 7$ & 17.6 & SII1253.8 \\
\hline FAIRALL9 & $1260.338 \pm 0.029$ & $199 \pm 7$ & $15 \pm 5$ & $34 \pm 9$ & 8.9 & SII1259.5 \\
\hline FAIRALL9 & $1261.039 \pm 0.035$ & $128 \pm 8$ & $54 \pm 6$ & $379 \pm 75$ & 105.2 & SiII+FeII1260.5 \\
\hline FAIRALL9 & $1261.300 \pm 0.028$ & $190 \pm 7$ & $36 \pm 4$ & $180 \pm 62$ & 51.3 & $\mathrm{SiII}+\mathrm{FeII} 1260.5$ \\
\hline $\mathrm{H} 1821+643$ & $1238.608 \pm 0.029$ & $-47 \pm 7$ & $38 \pm 22$ & $42 \pm 27$ & 5.9 & NV1238.8 \\
\hline $\mathrm{H} 1821+643$ & $1242.616 \pm 0.029$ & $-44 \pm 7$ & $42 \pm 20$ & $56 \pm 30$ & 8.2 & NV1242.8 \\
\hline $\mathrm{H} 1821+643$ & $1250.314 \pm 0.029$ & $-65 \pm 7$ & $26 \pm 13$ & $30 \pm 17$ & 4.4 & SII1250.6 \\
\hline $\mathrm{H} 1821+643$ & $1253.314 \pm 0.029$ & $-119 \pm 7$ & $17 \pm 10$ & $31 \pm 13$ & 5.0 & SII1253.8 \\
\hline $\mathrm{H} 1821+643$ & $1259.157 \pm 0.029$ & $-86 \pm 7$ & $59 \pm 10$ & $99 \pm 24$ & 16.2 & SII1259.5 \\
\hline $\mathrm{H} 1821+643$ & $1260.052 \pm 0.029$ & $-107 \pm 7$ & $59 \pm 4$ & $440 \pm 35$ & 74.4 & $\mathrm{SiII}+\mathrm{FeII} 1260.5$ \\
\hline MARK279 & $1238.260 \pm 0.037$ & $-136 \pm 9$ & $20 \pm 18$ & $22 \pm 16$ & 4.3 & NV1238.8 \\
\hline MARK279 & $1249.933 \pm 0.034$ & $-160 \pm 8$ & $23 \pm 14$ & $12 \pm 8$ & 4.0 & SII1250.6 \\
\hline MARK290 & $1250.019 \pm 0.029$ & $-139 \pm 7$ & $31 \pm 22$ & $19 \pm 23$ & 5.6 & SII1250.6 \\
\hline MARK290 & $1253.327 \pm 0.029$ & $-113 \pm 7$ & $28 \pm 5$ & $34 \pm 6$ & 13.7 & SII1253.8 \\
\hline MARK290 & $1259.058 \pm 0.029$ & $-110 \pm 7$ & $39 \pm 7$ & $72 \pm 16$ & 15.2 & SII1259.5 \\
\hline MARK290 & $1260.008 \pm 0.029$ & $-117 \pm 7$ & $63 \pm 3$ & $479 \pm 28$ & 97.3 & SiII+FeII1260.5 \\
\hline MARK421 & $1250.385 \pm 0.031$ & $-52 \pm 7$ & $11 \pm 16$ & $28 \pm 31$ & 4.5 & SII1250.6 \\
\hline MARK421 & $1253.578 \pm 0.031$ & $-53 \pm 8$ & $11 \pm 13$ & $33 \pm 29$ & 4.8 & SII1253.8 \\
\hline *MARK501 & $1253.069 \pm 0.264$ & $-175 \pm 63$ & $99 \pm 3$ & $64 \pm 33$ & 4.7 & SII1253.8 \\
\hline MARK509 & $1239.182 \pm 0.029$ & $-180 \pm 7$ & $21 \pm 18$ & $24 \pm 29$ & 5.1 & MgII1239.9 \\
\hline MARK509 & $1243.060 \pm 0.734$ & $62 \pm 177$ & $35 \pm 38$ & $17 \pm 21$ & 4.3 & NV1242.8 \\
\hline MARK509 & $1250.314 \pm 0.029$ & $-69 \pm 7$ & $24 \pm 9$ & $20 \pm 7$ & 7.2 & SII1250.6 \\
\hline MARK509 & $1250.819 \pm 0.029$ & $56 \pm 7$ & $42 \pm 5$ & $58 \pm 8$ & 21.3 & SII1250.6 \\
\hline MARK509 & $1253.561 \pm 0.029$ & $-57 \pm 7$ & $41 \pm 9$ & $27 \pm 7$ & 11.7 & SII1253.8 \\
\hline MARK509 & $1254.079 \pm 0.032$ & $64 \pm 8$ & $40 \pm 6$ & $85 \pm 19$ & 37.5 & SII1253.8 \\
\hline *MARK509 & $1258.365 \pm 0.029$ & $-270 \pm 7$ & $53 \pm 13$ & $237 \pm 151$ & 122.7 & SiII1259.5 \\
\hline *MARK509 & $1258.565 \pm 0.029$ & $-222 \pm 7$ & $33 \pm 8$ & $43 \pm 46$ & 22.6 & SiII1259.5 \\
\hline *MARK509 & $1259.858 \pm 0.029$ & $85 \pm 7$ & $34 \pm 19$ & $30 \pm 37$ & 16.1 & SII1259.5 \\
\hline
\end{tabular}


Table 7-Continued

\begin{tabular}{lcccccl}
\hline \hline Target & $\begin{array}{c}\text { Wavelength } \\
(\AA)\end{array}$ & $\begin{array}{c}\text { Velocity } \\
\left(\mathrm{km} \mathrm{s}^{-1}\right)\end{array}$ & $\begin{array}{c}b \\
\left(\mathrm{~km} \mathrm{~s}^{-1}\right)\end{array}$ & $\begin{array}{c}\mathcal{W} \\
(\mathrm{m} \AA)\end{array}$ & $\begin{array}{c}\text { SL } \\
(\sigma)\end{array}$ & \multicolumn{1}{c}{ ID } \\
\hline *MARK509 & $1260.257 \pm 0.029$ & $-48 \pm 7$ & $68 \pm 64$ & $43 \pm 52$ & 22.2 & SiII+FeII1260.5 \\
*MARK509 & $1260.710 \pm 0.029$ & $50 \pm 7$ & $72 \pm 4$ & $372 \pm 73$ & 186.9 & SiII+FeII1260.5 \\
MARK817 & $1238.529 \pm 0.029$ & $-65 \pm 7$ & $75 \pm 28$ & $54 \pm 41$ & 13.5 & NV1238.8 \\
MARK817 & $1239.509 \pm 0.029$ & $-101 \pm 7$ & $46 \pm 18$ & $25 \pm 12$ & 6.3 & MgII1239.9 \\
MARK817 & $1250.071 \pm 0.034$ & $-127 \pm 8$ & $24 \pm 13$ & $13 \pm 6$ & 5.1 & SII1250.6 \\
MARK817 & $1250.415 \pm 0.029$ & $-44 \pm 7$ & $30 \pm 11$ & $26 \pm 9$ & 9.8 & SII1250.6 \\
MARK817 & $1253.355 \pm 0.027$ & $-106 \pm 6$ & $27 \pm 12$ & $32 \pm 13$ & 12.9 & SII1253.8 \\
MARK817 & $1253.653 \pm 0.029$ & $-35 \pm 7$ & $27 \pm 8$ & $53 \pm 16$ & 21.7 & SII1253.8 \\
PKS2155-304 & $1259.848 \pm 0.028$ & $-155 \pm 7$ & $31 \pm 9$ & $76 \pm 23$ & 8.5 & SiII+FeII1260.5 \\
\hline
\end{tabular}


Table 8. Intrinsic Ly $\alpha$ features with $S L \geq 4 \sigma$.

\begin{tabular}{|c|c|c|c|c|c|c|}
\hline Target & $\begin{array}{c}\text { Wavelength } \\
(\AA)\end{array}$ & $\begin{array}{l}\text { Velocity } \\
\left(\mathrm{km} \mathrm{s}^{-1}\right)\end{array}$ & $\begin{array}{c}b \\
\left(\mathrm{~km} \mathrm{~s}^{-1}\right)\end{array}$ & $\begin{array}{c}\mathcal{W} \\
(\mathrm{m} \AA)\end{array}$ & $\begin{array}{l}\mathrm{SL} \\
(\sigma)\end{array}$ & $\mathrm{z}$ \\
\hline AKN120 & $1252.135 \pm 0.034$ & $-907 \pm 8$ & $28 \pm 11$ & $18 \pm 7$ & 5.1 & $0.03000 \pm 0.00003$ \\
\hline ESO141-G55 & $1257.716 \pm 0.029$ & $-733 \pm 7$ & $53 \pm 38$ & $18 \pm 20$ & 5.7 & $0.03459 \pm 0.00002$ \\
\hline ESO141-G55 & $1258.093 \pm 0.023$ & $-643 \pm 5$ & $46 \pm 3$ & $353 \pm 11$ & 116.3 & $0.03490 \pm 0.00002$ \\
\hline FAIRALL9 & $1268.850 \pm 0.029$ & $-688 \pm 7$ & $81 \pm 22$ & $32 \pm 12$ & 11.2 & $0.04375 \pm 0.00002$ \\
\hline FAIRALL9 & $1269.351 \pm 0.029$ & $-570 \pm 7$ & $46 \pm 19$ & $16 \pm 9$ & 5.4 & $0.04416 \pm 0.00002$ \\
\hline FAIRALL9 & $1269.731 \pm 0.029$ & $-480 \pm 7$ & $99 \pm 3$ & $74 \pm 7$ & 26.7 & $0.04447 \pm 0.00002$ \\
\hline FAIRALL9 & $1270.288 \pm 0.032$ & $-349 \pm 8$ & $89 \pm 12$ & $93 \pm 16$ & 32.2 & $0.04493 \pm 0.00003$ \\
\hline FAIRALL9 & $1270.746 \pm 0.027$ & $-241 \pm 6$ & $11 \pm 11$ & $11 \pm 6$ & 4.2 & $0.04531 \pm 0.00002$ \\
\hline FAIRALL9 & $1270.955 \pm 0.029$ & $-191 \pm 7$ & $42 \pm 5$ & $55 \pm 9$ & 20.3 & $0.04548 \pm 0.00002$ \\
\hline MARK279 & $1249.023 \pm 0.036$ & $-923 \pm 9$ & $43 \pm 12$ & $22 \pm 8$ & 7.5 & $0.02744 \pm 0.00003$ \\
\hline MARK279 & $1251.056 \pm 0.025$ & $-436 \pm 6$ & $99 \pm 3$ & $646 \pm 18$ & 240.5 & $0.02911 \pm 0.00002$ \\
\hline MARK279 & $1251.261 \pm 0.029$ & $-387 \pm 7$ & $19 \pm 10$ & $17 \pm 10$ & 6.6 & $0.02928 \pm 0.00002$ \\
\hline MARK279 & $1251.694 \pm 0.023$ & $-284 \pm 5$ & $71 \pm 3$ & $322 \pm 14$ & 123.9 & $0.02963 \pm 0.00002$ \\
\hline MARK279 & & & & & & $0.03075 \pm 0.00002$ \\
\hline MARK279 & $1253.284 \pm 0.031$ & $97 \pm 7$ & $51 \pm 12$ & $375 \pm 117$ & 146.8 & $0.03094 \pm 0.00003$ \\
\hline MARK279 & $1253.861 \pm 0.040$ & $235 \pm 10$ & $26 \pm 6$ & $94 \pm 53$ & 35.4 & $0.03142 \pm 0.00003$ \\
\hline MARK290 & $1248.079 \pm 0.028$ & $-850 \pm 7$ & $20 \pm 9$ & $22 \pm 9$ & 4.9 & $0.02666 \pm 0.00002$ \\
\hline MARK290 & $1249.349 \pm 0.029$ & $-545 \pm 7$ & $50 \pm 26$ & $25 \pm 23$ & 6.4 & $0.02770 \pm 0.00002$ \\
\hline MARK290 & $1249.579 \pm 0.029$ & $-490 \pm 7$ & $97 \pm 12$ & $225 \pm 48$ & 60.4 & $0.02789 \pm 0.00002$ \\
\hline MARK290 & $1251.115 \pm 0.039$ & $-122 \pm 9$ & $99 \pm 3$ & $475 \pm 37$ & 185.6 & $0.02916 \pm 0.00003$ \\
\hline MARK290 & $1251.664 \pm 0.025$ & & & & 45.2 & $\leftarrow 0.00002$ \\
\hline MARK290 & $1252.109 \pm 0.029$ & $116 \pm 7$ & $61 \pm 12$ & $71 \pm 17$ & 31.3 & $0.02997 \pm 0.00002$ \\
\hline MARK290 & $1252.479 \pm 0.029$ & $204 \pm 7$ & $35 \pm 6$ & $33 \pm 10$ & 14.5 & $0.03028 \pm 0.00002$ \\
\hline MARK290 & $1254.353 \pm 0.029$ & $653 \pm 7$ & $81 \pm 29$ & $50 \pm 24$ & 17.3 & $0.03182 \pm 0.00002$ \\
\hline MARK290 & $1254.653 \pm 0.029$ & $725 \pm 7$ & $35 \pm 14$ & $24 \pm 12$ & 7.8 & $0.03207 \pm 0.00002$ \\
\hline MARK290 & $1254.953 \pm 0.029$ & $797 \pm 7$ & $23 \pm 13$ & $16 \pm 10$ & 4.8 & $0.03231 \pm 0.00002$ \\
\hline *MARK501 & $1254.773 \pm 0.029$ & $-434 \pm 7$ & $99 \pm 3$ & $70 \pm 28$ & 5.4 & $0.03217 \pm 0.00002$ \\
\hline *MARK509 & $1255.795 \pm 0.029$ & $-403 \pm 7$ & $46 \pm 5$ & $132 \pm 67$ & 64.4 & $0.03301 \pm 0.00002$ \\
\hline *MARK509 & $1256.065 \pm 0.029$ & $-339 \pm 7$ & $44 \pm 14$ & $76 \pm 92$ & 37.9 & $0.03323 \pm 0.00002$ \\
\hline *MARK509 & $1256.285 \pm 0.029$ & $-286 \pm 7$ & $93 \pm 31$ & $505 \pm 425$ & 147.7 & $0.03341 \pm 0.00002$ \\
\hline *MARK509 & $1257.165 \pm 0.029$ & $-76 \pm 7$ & $69 \pm 21$ & $191 \pm 229$ & 61.9 & $0.03413 \pm 0.00002$ \\
\hline *MARK509 & $1257.365 \pm 0.029$ & $-29 \pm 7$ & $65 \pm 62$ & $265 \pm 318$ & 121.5 & $0.03430 \pm 0.00002$ \\
\hline
\end{tabular}


Table 8 -Continued

\begin{tabular}{lcrcccc}
\hline \hline Target & $\begin{array}{c}\text { Wavelength } \\
(\AA)\end{array}$ & $\begin{array}{c}\text { Velocity } \\
\left(\mathrm{km} \mathrm{s}^{-1}\right)\end{array}$ & $\begin{array}{c}b \\
\left(\mathrm{~km} \mathrm{~s}^{-1}\right)\end{array}$ & $\begin{array}{c}\mathcal{W} \\
(\mathrm{m} \AA)\end{array}$ & $\begin{array}{c}\text { SL } \\
(\sigma)\end{array}$ & z \\
\hline *MARK509 & $1257.605 \pm 0.029$ & $29 \pm 7$ & $44 \pm 16$ & $140 \pm 168$ & 73.7 & $0.03450 \pm 0.00002$ \\
*MARK509 & $1257.785 \pm 0.029$ & $71 \pm 7$ & $28 \pm 17$ & $26 \pm 32$ & 13.9 & $0.03464 \pm 0.00002$ \\
*MARK509 & $1257.985 \pm 0.029$ & $119 \pm 7$ & $72 \pm 25$ & $449 \pm 240$ & 238.6 & $0.03481 \pm 0.00002$ \\
MARK817 & $1249.477 \pm 0.041$ & $-1023 \pm 10$ & $26 \pm 9$ & $36 \pm 17$ & 13.8 & $0.02781 \pm 0.00003$ \\
MARK817 & $1249.694 \pm 0.077$ & $-971 \pm 18$ & $28 \pm 21$ & $20 \pm 19$ & 7.7 & $0.02799 \pm 0.00006$ \\
MARK817 & $1251.752 \pm 0.025$ & $-479 \pm 6$ & $18 \pm 7$ & $14 \pm 4$ & 6.0 & $0.02968 \pm 0.00002$ \\
\hline
\end{tabular}




\section{CfA Galaxy pie diagrams}

The very local redshift range of this absorption line survey $\left(c z \leq 20,000 \mathrm{~km} \mathrm{~s}^{-1}\right)$, allows us to present an immediate "first look" at the relationship between local Ly $\alpha$ absorbers and galaxies using bright galaxy redshift information currently available. Several surveys are complete to $m_{B} \leq 15.5$ over large sky areas (e.g., Huchra et al. 1992; Marzke, Huchra, \& Geller 1996; Grogin \& Geller 1998; Huchra, Vogeley, \& Geller 1999; Da Costa et al. 1998). Seven of our targets lie in the sky areas of these surveys (3C 273, IZW1, Mrk 335, Mrk 421, Mrk 501, Q 1230+011 and PKS 2155-304). All of these redshifts surveys are included in the latest (Feb 8, 2000) on-line version of the CfA Redshift Catalogue (Huchra et al. 1992) so that the environments of the Ly $\alpha$ absorbers found along these sightlines can be investigated using available data. Also, pencil-beam surveys have been conducted along several sightlines to address the relationship between galaxies and Ly $\alpha$ clouds using smaller numbers of absorbers than presented herein (e.g., Morris et al. 1993; Tripp, Lu, \& Savage 1998a). In Paper III, we will use our Ly $\alpha$ survey and all available galaxy redshift survey data to investigate the relationship between $\operatorname{Ly} \alpha$ clouds and galaxies. One advantage of the current work is that the absorber redshifts are known to much better accuracy (i.e., $\pm 10 \mathrm{~km} \mathrm{~s}^{-1}$ compared to $\pm 200 \mathrm{~km} \mathrm{~s}^{-1}$ ) than the absorbers detected in the QSO Absorption Line Key Project, which used the FOS at $1 \AA$ resolution.

In Figures 21-35, we present heliocentric "pie diagrams" (right ascension [RA, $\alpha]$ or declination [DEC, $\delta]$ versus heliocentric radial velocity) for all 15 HST targets in our GHRS survey. These pie diagrams indicate the spatial positions of the CfA Redshift Catalogue galaxies relative to our target sightlines and the detected Ly $\alpha$ absorbers, assuming a pure Hubble flow. The 2000 February 8 version of this catalog (Huchra et al. 1992) contains $\sim 120,000$ galaxies with velocities less than $100,000 \mathrm{~km} \mathrm{~s}^{-1}$. We present pie diagram in $\pm 7^{\circ}$ extent as a $2 \mathrm{D}$ representation of the $3 \mathrm{D}$ distribution of the known low- $z$ galaxy distribution. The sightline towards each target is indicated by the dashed line that starts at the apex of each pie diagram (the Sun) and terminates with either a large circle, indicating the position of the HST target, or with a dashed arrow indicating that target lies off the pie diagram. Each CfA galaxy is indicated by a "c". CfA galaxies that have a sightline impact parameter less than $1 h_{70}^{-1} \mathrm{Mpc}$ are indicated by "v"s (the $\mathbf{v}$ indicates that the galaxy is very close to the sightline). The orientation of the ' $\mathbf{v}$ 's and 'c's are preserved between the RA and DEC versions of the pie diagrams.

In the previous sections, all Ly $\alpha$ absorber velocities were reported in the LSR velocity scale, established by aligning the velocity centroids of the Galactic S II $\lambda \lambda 1250,1253,1259$ absorption lines with the dominant Galactic H I emission. We selected this velocity scale instead of the HST+GHRS wavelength solution due to the possibility of an improper wavelength scale caused by poor target centering in the large science aperture (LSA). To convert our LSR Ly $\alpha$ absorber velocities to heliocentric velocities, we assumed that the solar velocity with respect to LSR is

$+20.0 \mathrm{~km} \mathrm{~s}^{-1}$ towards $(\alpha=18: 03: 50.3 ; \delta=+30: 00: 17$, J2000). The heliocentric velocities of our definite Ly $\alpha$ absorbers $(S L \geq 4 \sigma)$, if present, are indicated on the pie diagrams by medium-sized 
dashed circles. Possible Ly $\alpha$ absorbers $(3 \sigma \leq S L<4 \sigma)$, if present, are indicated by small dashed circles. If the target is present within the pie diagram, its location is given by a large circle; otherwise a dashed arrow indicates that the target is beyond the limit of the pie diagram.

Also in Appendix B, Table 10 lists the nearest 3 galaxies for all Ly $\alpha$ absorbers in our $S L \geq 3 \sigma$ sample. For each absorber, we indicate absorber type $(\mathrm{D}=$ definite, or $\mathrm{P}=$ possible), heliocentric absorber velocity in $\mathrm{km} \mathrm{s}^{-1}$, name of the nearest galaxy, distance $\left(\mathrm{D}_{\perp}\right)$ from the galaxy to the line of sight (LOS) in $h_{70}^{-1} \mathrm{Mpc}$, heliocentric recession velocity $(c z)$ of the galaxy, distance $\left(\mathrm{D}_{c z}\right)$ along the LOS from the absorber to the galaxy allowing for peculiar velocities of $\pm 300 \mathrm{~km} \mathrm{~s}^{-1}$, the Euclidean 3D absorber-galaxy distance $\left(\mathrm{D}_{\text {tot }}\right)$, and the Zwicky blue $(\mathrm{B})$ magnitude of the galaxy (if available). All information on galaxy location, velocity, or magnitude is taken from the CfA redshift survey unless otherwise indicated. The use of a "retarded" Hubble flow accounts for peculiar galaxy motion and galaxy rotation and always decreases the reported LOS and total absorbergalaxy distances. With our "retarded Hubble flow" peculiar velocity allowance, we consider any Galaxy within $\pm 300 \mathrm{~km} \mathrm{~s}^{-1}$ of the recession velocity of an absorber to be at the same distance as the absorber (see S95 for justification of this somewhat arbitrary $\pm 300 \mathrm{~km} \mathrm{~s}^{-1}$ allowance). In Paper III, we will use the data presented here to quantify the environment of local Ly $\alpha$ clouds.

\section{Conclusion}

This paper is the first in a series devoted to a detailed study of the "local Ly $\alpha$ forest" using observations obtained with the Hubble Space Telescope (HST) and Goddard High Resolution Spectrometer (GHRS) in its medium resolution mode (G160M grating). The current paper has presented a detailed description of the target selection, observational strategy and data reduction $(\S 2)$, along with a presentation of the reduced spectra and lists of the all absorption lines detected ( $\$ 4$ and Appendix A). We discovered 81 definite $(\geq 4 \sigma)$ and 30 possible $(3-4 \sigma)$ intervening Ly $\alpha$ absorption lines along with numerous Galactic metal lines, including metal absorption from several "high-velocity clouds", and a few absorption systems judged to be "intrinsic" to the target AGN. In Paper II of this series we will present a detailed analysis of the absorption line data presented herein, including the " $b$-value" distribution, the column density distribution, and the two-point correlation function for local Ly $\alpha$ absorbers. In Paper III we will present an analysis of the relationship between these $\operatorname{Ly} \alpha$ absorbing clouds and galaxies based upon the bright galaxy survey data also presented herein. The low- $z$ galaxy redshift survey data presented in the form of "pie diagrams" in $\S 5$ and Appendix B provide a valuable "first look" at the relationship between the local Ly $\alpha$ clouds and bright galaxies. In Paper IV we will present the two-point correlation function of the low- $z$ Ly $\alpha$ forest based upon the HST/GHRS data presented here and 13 additional HST/STIS sightlines obtained in Cycle 7.

The GHRS+G160M observing combination has two primary advantages for doing this study: (1) it is sensitive to the weakest Ly $\alpha$ absorbers detected at low- $z$ thus far $\left(\log N_{\mathrm{HI}} \geq 12.5\right.$; comparable to the weakest lines found in the high-z Ly $\alpha$ forest with the Keck Telescope); and (2) the 
$\sim 19 \mathrm{~km} \mathrm{~s}^{-1}$ spectral resolution resolves almost all of the Ly $\alpha$ lines detected. This combination also yields extremely accurate velocities for detected absorbers $\left(\leq 10 \mathrm{~km} \mathrm{~s}^{-1}\right)$. The disadvantages of this approach are that only the UV-brightest AGN in the sky are possible targets for this work (typically $V \leq 14.5$ ) and that only a small pathlength can be observed with this spectrograph configuration. The fifteen GHRS target sightlines sample only $116,000 \mathrm{~km} \mathrm{~s}^{-1}$ of total pathlength for Ly $\alpha$ absorber detection (details of the pathlength surveyed as a function of $\mathrm{H}$ I column density are presented in Paper II). However, the discovery of a large number of Ly $\alpha$ absorbers by this GHRS survey makes it clear that the observational approach adopted here of obtaining the highest resolution spectra possible to investigate the local Ly $\alpha$ forest has been successful. Furthermore, even though the total observed pathlength is small compared to other Ly $\alpha$ surveys, the pathlength observed by this work is the most important piece of any individual sightline, the nearest $\sim 10,000 \mathrm{~km} \mathrm{~s}^{-1}$. This has enabled the discovery of the nearest Ly $\alpha$ absorbers and given us the opportunity to relate these clouds to the nearest known galaxies.

Owing to the success of this GHRS survey, our group has obtained HST + Space Telescope Imaging Spectrograph (STIS) spectra of an additional 13 UV bright targets to complete our survey of the low- $z$, low- $N_{\mathrm{HI}}$ local Ly $\alpha$ forest. The STIS observations will be presented in a later paper.

For their assistance in obtaining the HST/GHRS data over several cycles, we are grateful to the staff at the Space Telescope Science Institute, particularly Ray Lucas. We would like to thank Brad Gibson for his assistance in deciphering the HVCs and his helpful discussions on $b$-values. In addition, we would like to thank Mark Giroux and Greg Bothun for their helpful comments on this manuscript. This work was supported by HST guest observer grant GO-06593.01-95A, the HST COS project (NAS5-98043), and by the Astrophysical Theory Program (NASA grant NAGW-766 and NSF grant AST96-17073). 


\section{A. GHRS/G160M Spectra and Absorption Line Lists}

In this Appendix we present composite HST/GHRS/G160M spectra, and line lists (Table 9) describing the detected absorption features. Descriptions of features in these data unique to individual objects are presented in $\S 3$ of the text.

The GHRS/G160M spectra, error vectors, and pathlength accountings are presented in Figures 5-20 for all targets. The upper panel of each figure presents the composite HST/GHRS/G160M spectrum and the $1 \sigma$ error values, which are plotted below the flux vector. As discussed in $\S 2.3 .5$, our list of intergalactic Ly $\alpha$ absorbers is restricted to those more than $1,200 \mathrm{~km} \mathrm{~s}^{-1}$ blueward of the target Ly $\alpha$ emission. This limit is indicated by the dashed vertical line in the upper and middle panels of the figures. All non-Galactic features redward of this line are classified as intrinsic absorbers. All otherwise unidentified absorption features blueward are taken to be intergalactic Ly $\alpha$ absorption features, and are indicated by a solid vertical line above the feature. Ly $\alpha$ absorbers with significance level (SL) $>4 \sigma$ (definite absorbers) are plotted with a longer vertical line than the $3 \sigma \leq S L<4 \sigma$ absorbers. Galactic and high-velocity cloud (HVC) absorption lines are similarly represented with a dotted line, intrinsic absorption lines with a dashed line, and intervening higher$z$ absorption lines with a dot-dashed line. The solid line is the global continuum fit as described in $\S 2.3 .2$.

Note specifically that in several cases where Ly $\alpha$ emission occurs in the observed band, the portion of the spectrum redward of the proximity limit (vertical dashed line) is excluded from the region best fitted by the continuum. Thus, any intrinsic or Galactic absorptions redward of the "proximity limit" may not have their EWs most accurately represented. A different continuum fitting procedure will be used to address these specific features in later publications. The middle panel shows the $4 \sigma$ rest-frame EW $(\mathcal{W})$ sensitivity detection limit, per resolution element, as a function of wavelength for each target. As with the upper panel, we include in our statistics only the portion of the spectrum that lies $>1,200 \mathrm{~km} \mathrm{~s}^{-1}$ blueward of the target Ly $\alpha$ emission. Finally, the bottom panel of each figure summarizes the available pathlength for inclusion in our sample and line statistics. The row marked ' $\mathbf{I}$ ' indicates the portion of the spectrum removed due to specific features intrinsic to the target or non-Ly $\alpha$ lines associated with known intervening systems at higher redshift not associated with the target AGN. The pathlength removed due to higher redshift intervening absorbers is indicated by a double line to differentiate it from that removed due to intrinsic absorption features. The other rows are marked as follows: ' $G$ ' indicates pathlength attributed to non-HVC Galactic features such as S II $\lambda \lambda 1250,1253,1259$, ' $\mathbf{H}$ ' indicates pathlength attributed to HVCs, ' $\mathbf{L}$ ' indicates the portion of the spectrum that is redward of $\mathrm{c}_{\mathrm{em}}{ }^{-}$ $1,200 \mathrm{~km} \mathrm{~s}^{-1}$, and ' $\mathbf{F}$ ' indicates those regions of the spectrum that are available for the detection of intergalactic Ly $\alpha$ absorbers. The ' $\mathbf{F}$ ' row also corrects for our inability to correctly detect and model features near the edge of our wavelength coverage; we remove 10 pixels on the edges of our wavelength coverage. Only for $\mathrm{H} 1821+643$ does the intrinsic/intervening ' $\mathrm{I}$ ' accounting remove any significant pathlength not already removed by the ' $\mathbf{L}$ ' row. 
For a few objects, the $\mathrm{c} z_{\mathrm{em}}-1,200 \mathrm{~km} \mathrm{~s}^{-1}$ "proximity limit" lies within the observed spectral band and is indicated by a dashed vertical line. For targets with strong intrinsic Ly $\alpha$ emission (Akn 120, ESO 141-G55, Fairall 9, Mrk 279, Mrk 290, Mrk 335, and Mrk 509), fluxes are scaled differently above and below this convenient breakpoint. For these spectra, the left axis corresponds to flux blueward of the proximity limit, while the right axis corresponds to flux redward of this limit.

The line lists for each individual spectrum are presented sequentially in Table 9 . The first column of Table 9 indicates the LSR-adjusted wavelength and wavelength uncertainty for each feature. The wavelength scale of each spectrum was corrected to the LSR using the Galactic H I and S II lines (1250, 1253, and $1259 \AA$, if available). The second column lists the non-relativistic velocity $\left(c z\right.$ in $\mathrm{km} \mathrm{s}^{-1}$ ) relative to the LSR. For Ly $\alpha$ absorbers judged to be intrinsic to the AGN, velocities are listed relative to the AGN narrow-line region (see $\S 2.3 .5$ ). For intervening non-Ly $\alpha$ absorbers, such as those found in $\mathrm{H} 1821+643$, this column reports the redshift $(z)$ of the absorber. We also provide velocity or redshift uncertainties, based upon the total wavelength uncertainties (see $\S$ 2.3.3). The third column provides the single-component Doppler widths $\left(b\right.$ in $\mathrm{km} \mathrm{s}^{-1}$ ), and uncertainties, for each feature as estimated from the Gaussian width $\left(\mathbf{W}_{G}\right)$. As explained previously, the Doppler widths have been corrected for the spectral resolution of the GHRS and the single-component Gaussian fits were restricted to the range $12<b_{\text {obs }}<100 \mathrm{~km} \mathrm{~s}^{-1}$. The fourth column lists the rest-frame $\mathrm{EW}(\mathcal{W}$ in $\mathrm{m} \AA)$ and its uncertainty for each absorption feature. This uncertainty includes both the statistical error of the $\chi^{2}$ fit and our conservative estimate of the systematic error in the continuum placement (see $\S 2.3 .2$ ). The fifth column indicates the significance level (SL in $\sigma$ ) of each feature. The SL is calculated by integrating the S/N (per $\mathrm{RE}$ ) of the best fit Gaussian for each feature. The final two columns present the identification (Id) and alternative identification (Alt Id) if applicable for each feature. Absorption lines that are determined not to be intergalactic Ly $\alpha$ have their identifications prefaced by g: (Galactic), h: (HVC), i: (intrinsic), or z: (intervening). Alternate line identifications that lie between $0.2-$ $0.4 \AA$ from the expected location are indicated as speculative by the inclusion of a ? following the identification. 

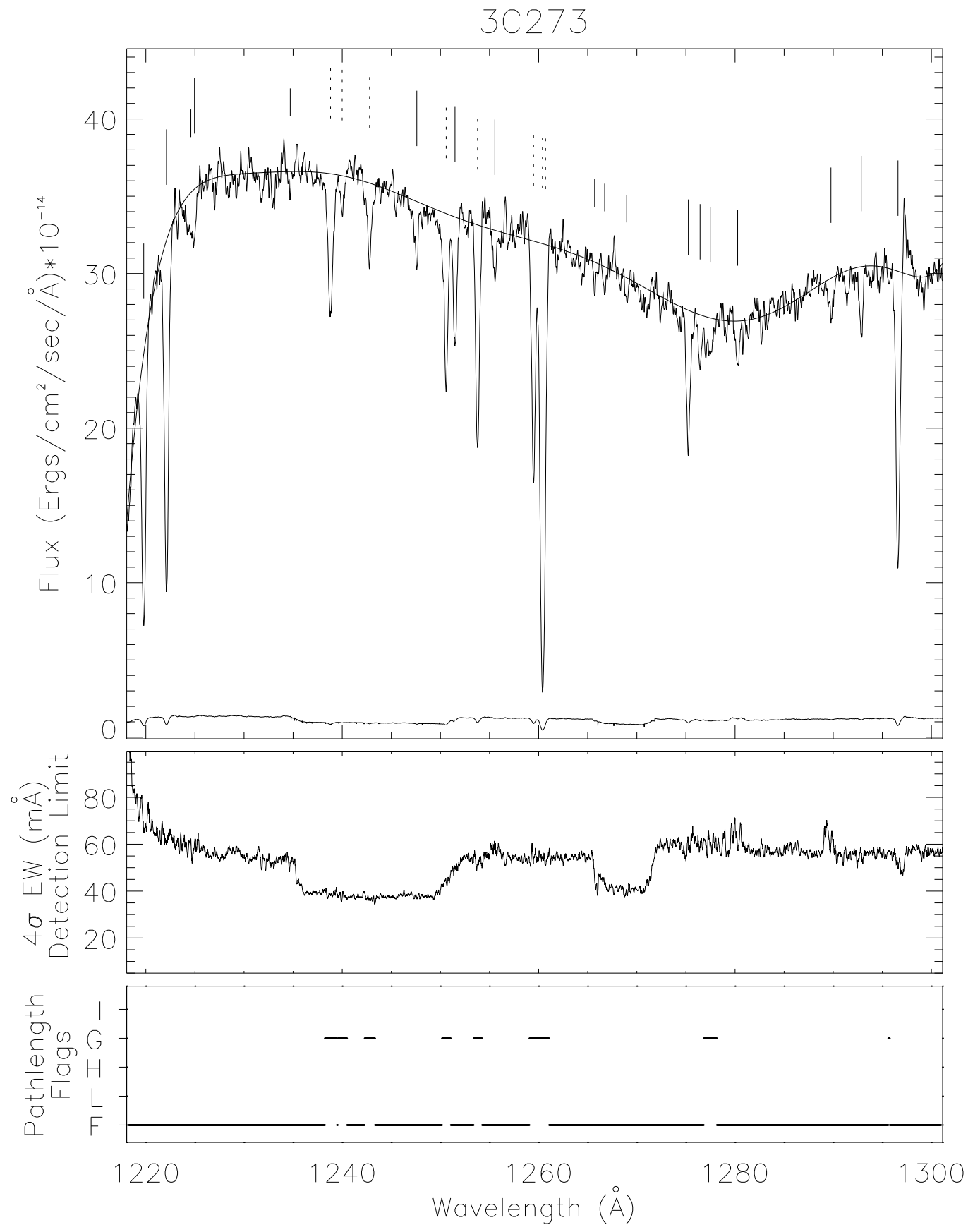

Fig. 5.- GHRS/G160M 3C 273 spectrum, sensitivity limits, and pathlength flags. 


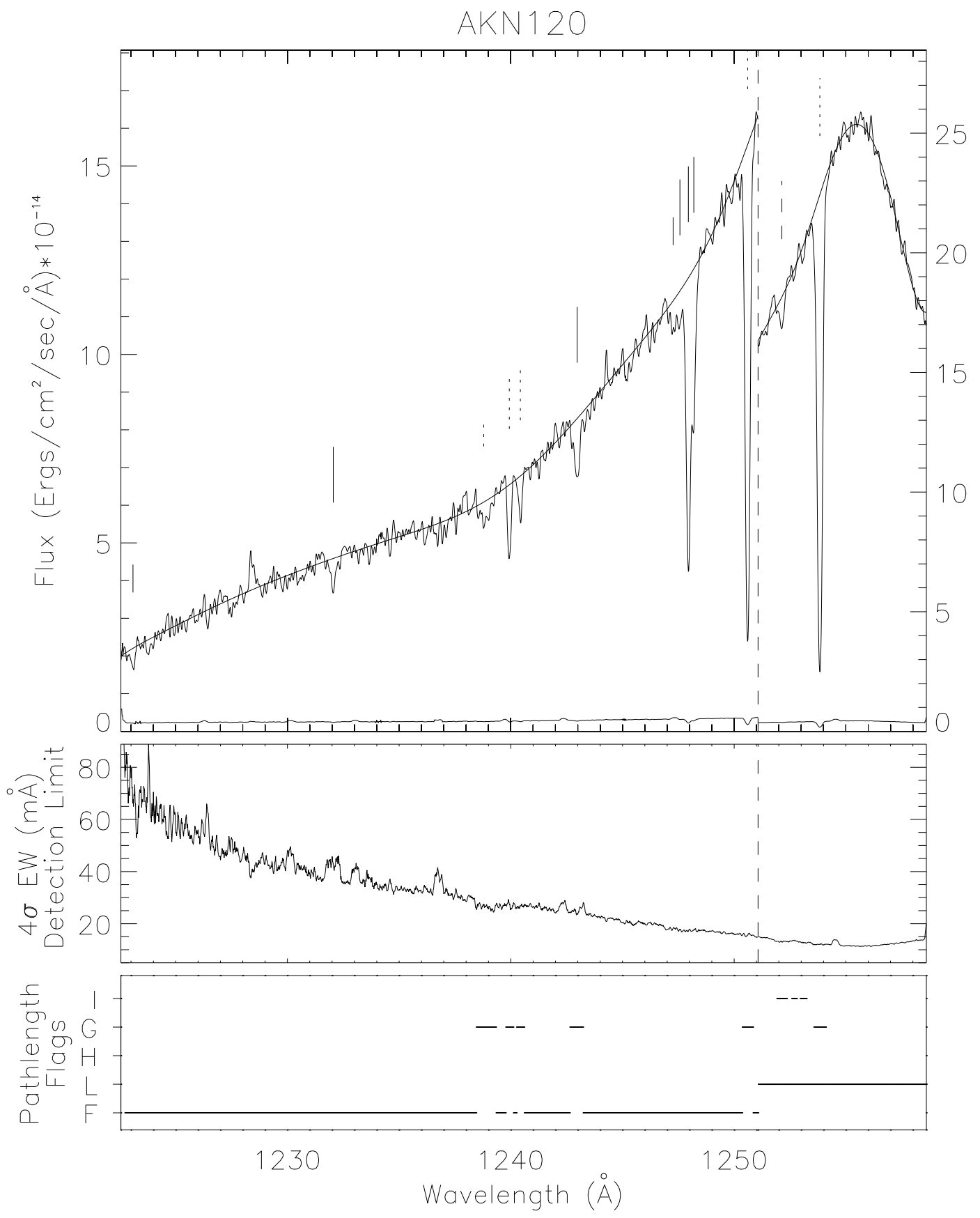

Fig. 6. - GHRS/G160M Arakelian 120 spectrum. The dashed vertical line indicates the proximity limit as explained in $\S$ 2.3.5. In the upper panel, the left axis corresponds to flux blueward of the proximity limit, while the right axis corresponds to flux redward of this convenient breakpoint. 


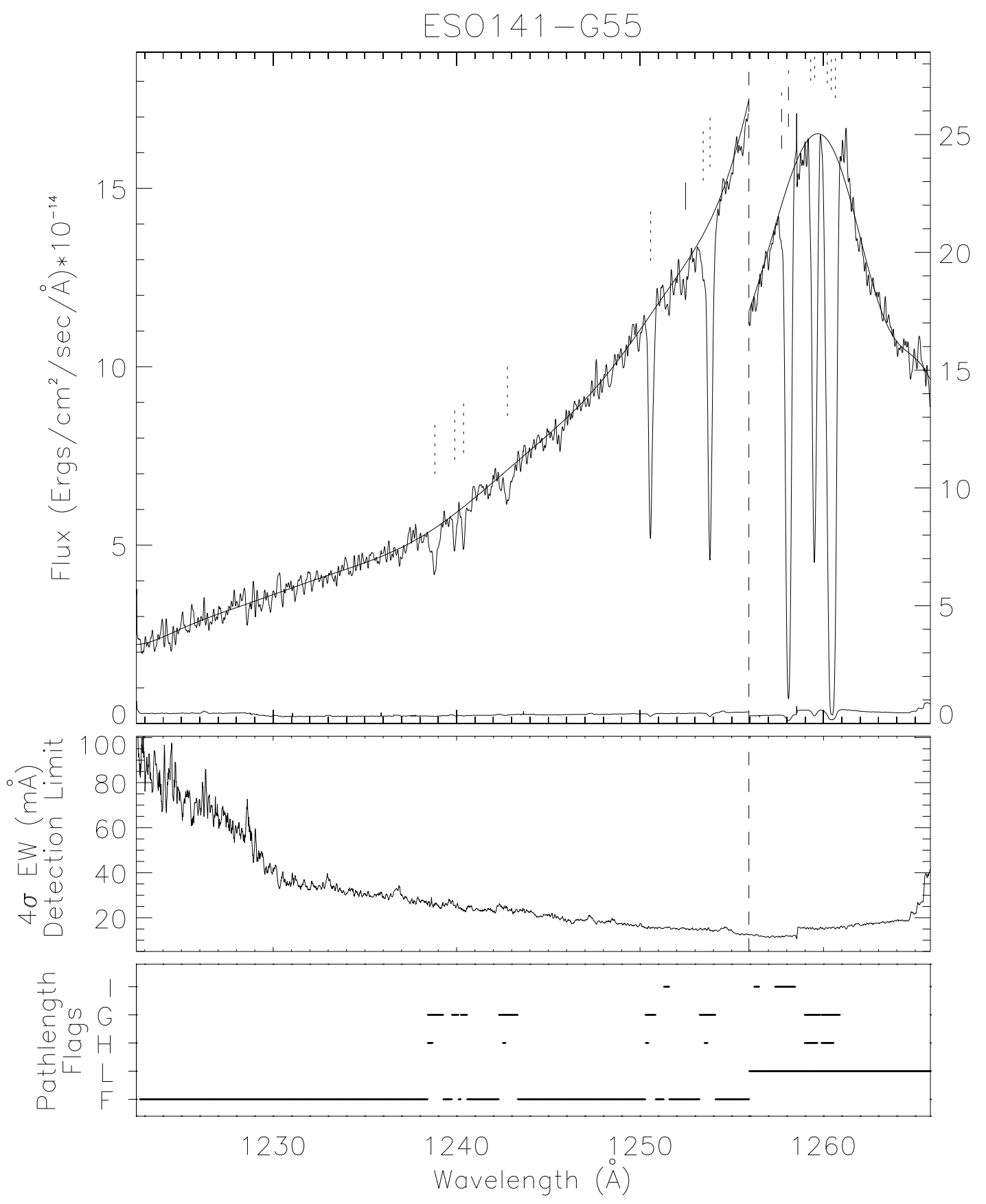

Fig. 7.- GHRS/G160M ESO 141-G55 spectrum, sensitivity limits, and pathlength flags. 


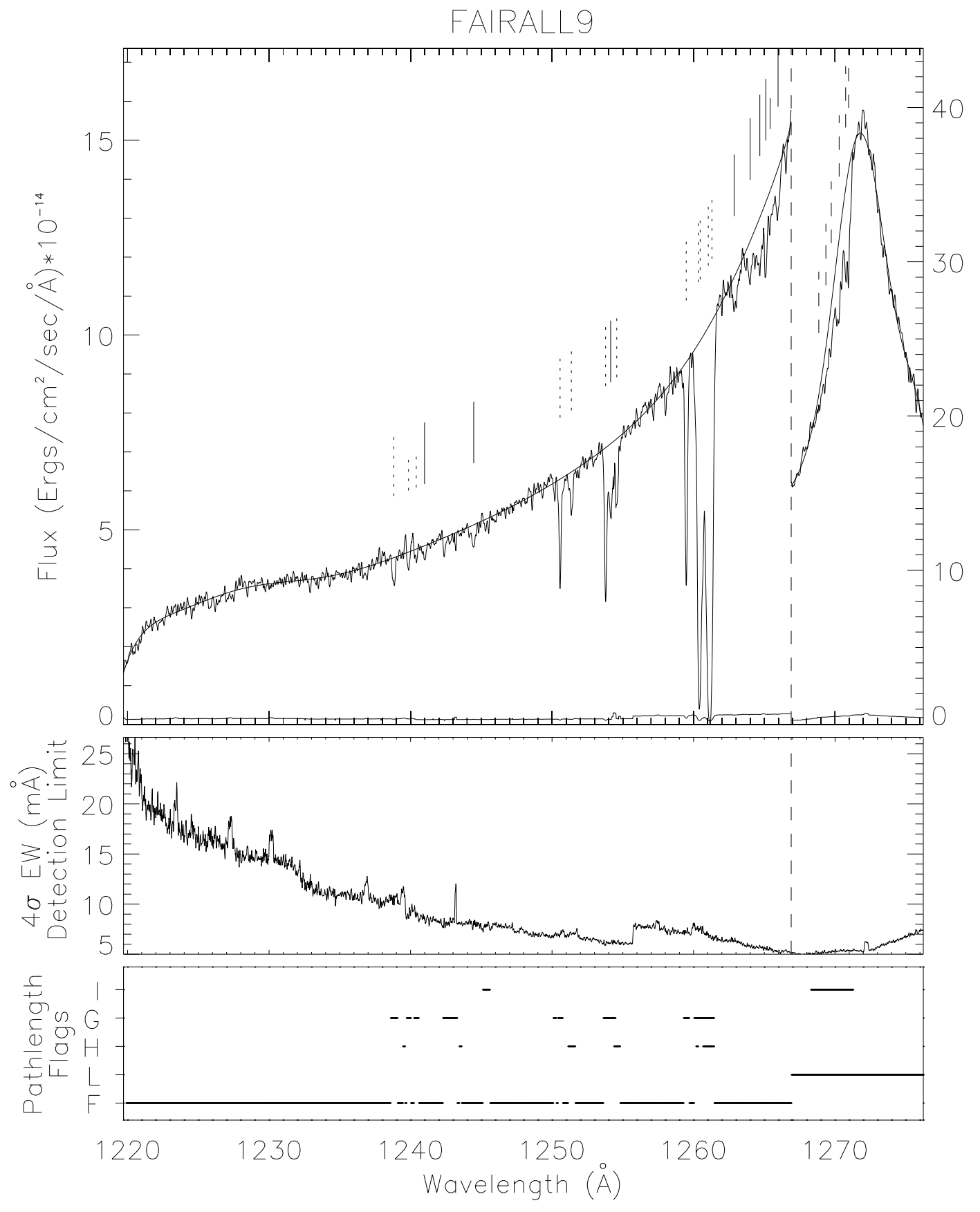

Fig. 8.- GHRS/G160M Fairall 9 spectrum, sensitivity limits, and pathlength flags. 


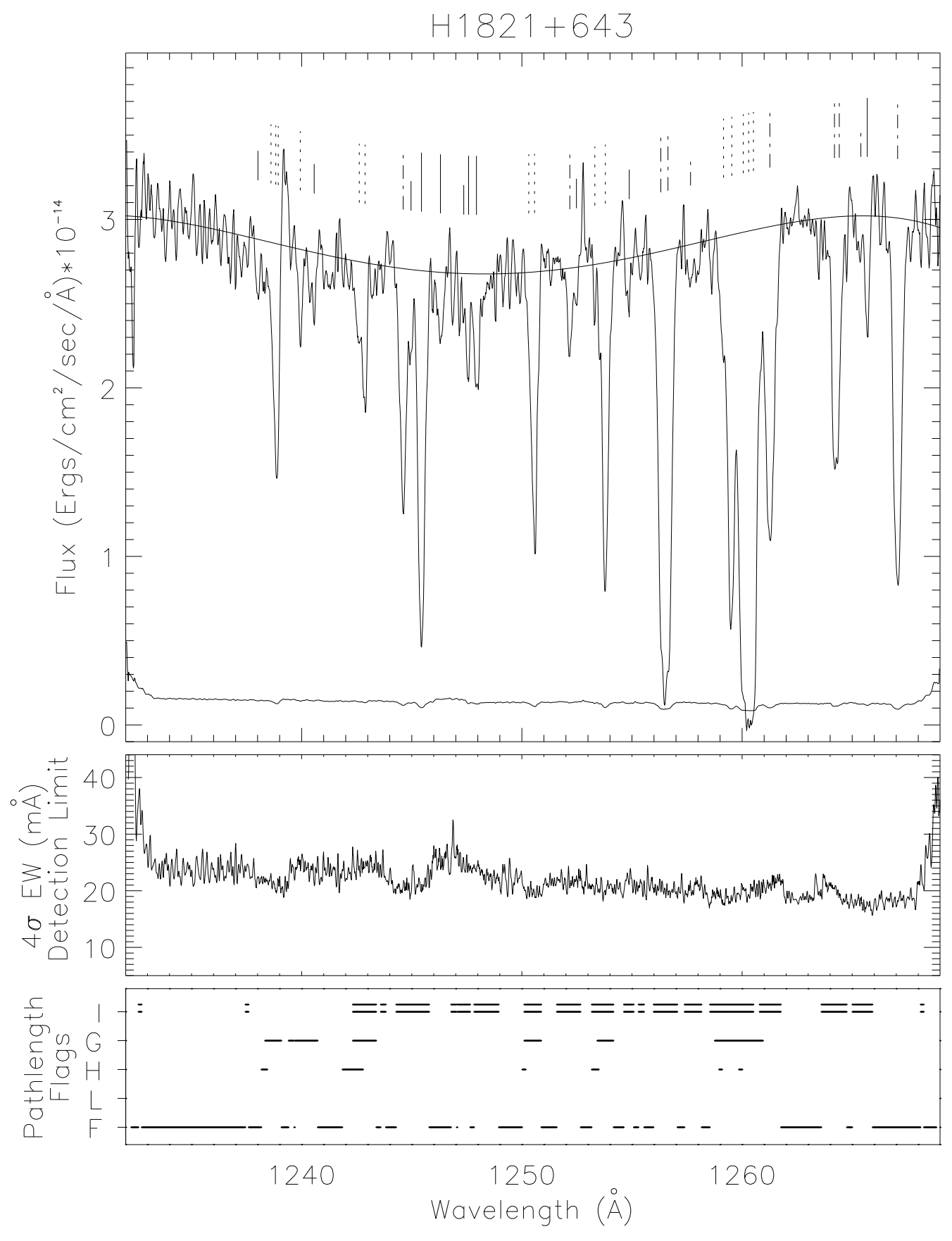

Fig. 9.- GHRS/G160M H 1821+643 spectrum. Features indicated in the upper panel by a dotdashed line are determined to be intergalactic non-Ly $\alpha$ lines. The double lines in the "I" row of the Pathlength Flags denotes pathlength excluded due to these intergalactic non-Ly $\alpha$ lines at higher $c z$. 


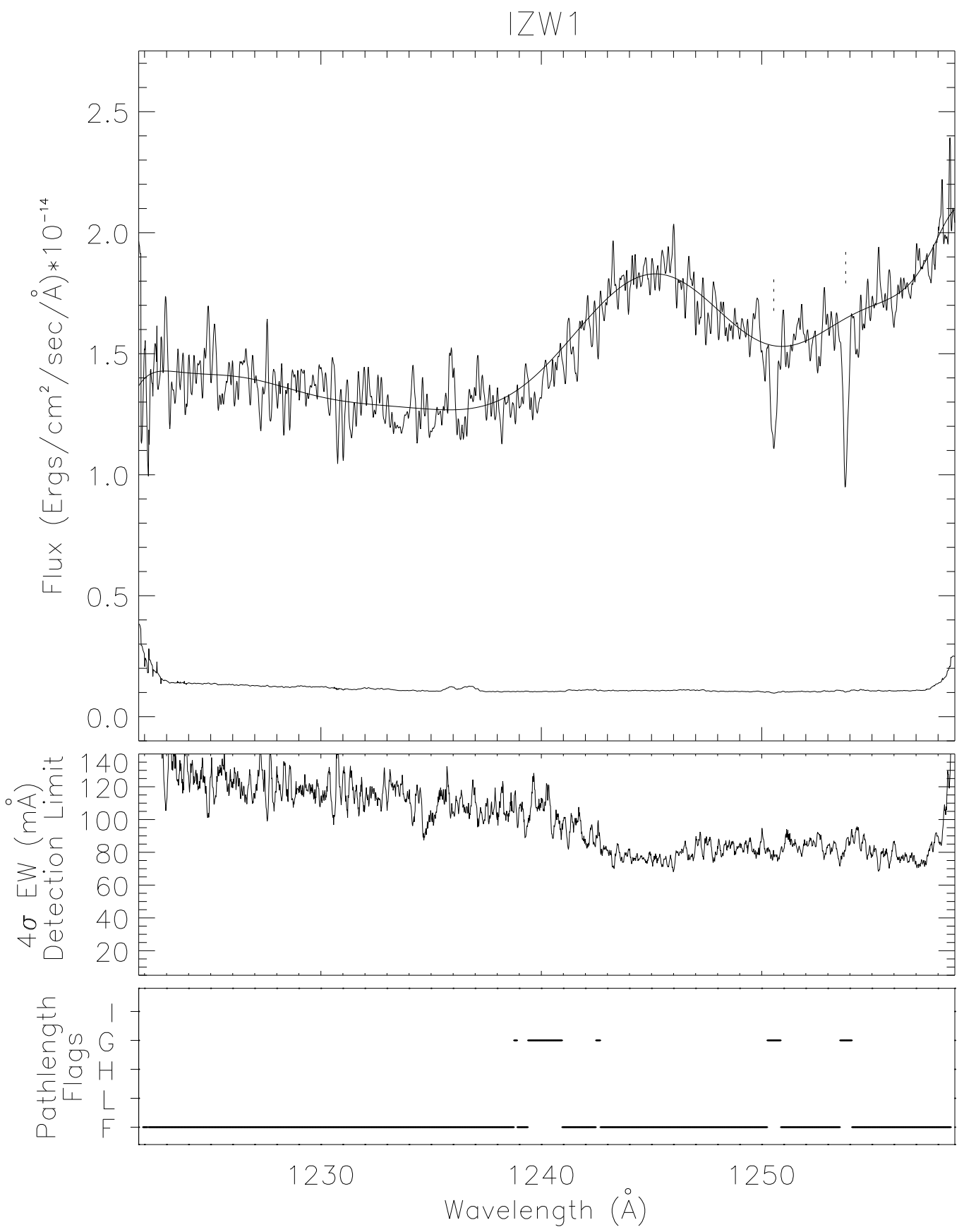

Fig. 10.- GHRS/G160M I ZW 1 spectrum, sensitivity limits, and pathlength flags. 

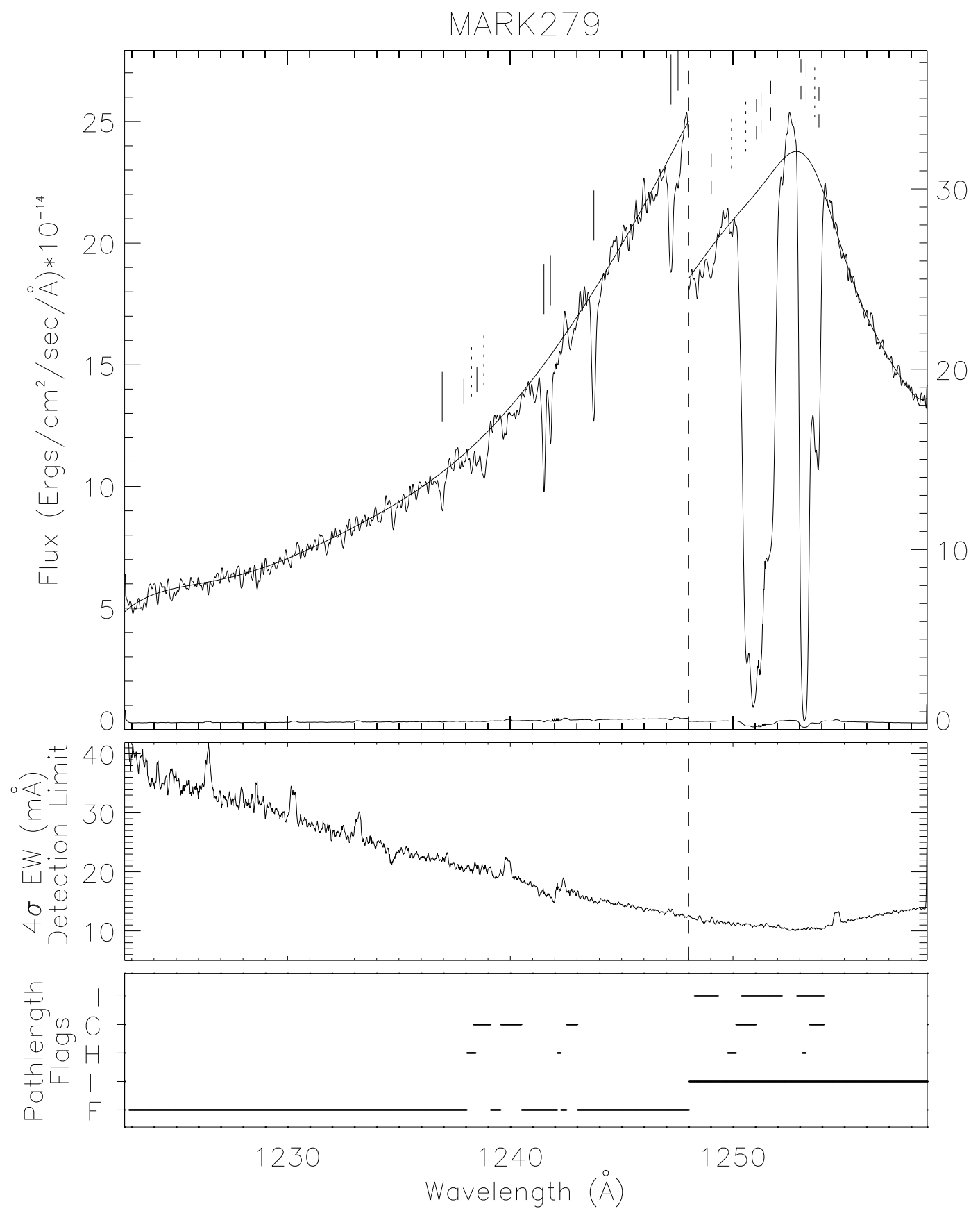

Fig. 11.- GHRS/G160M Markarian 279 spectrum, sensitivity limits, and pathlength flags. 

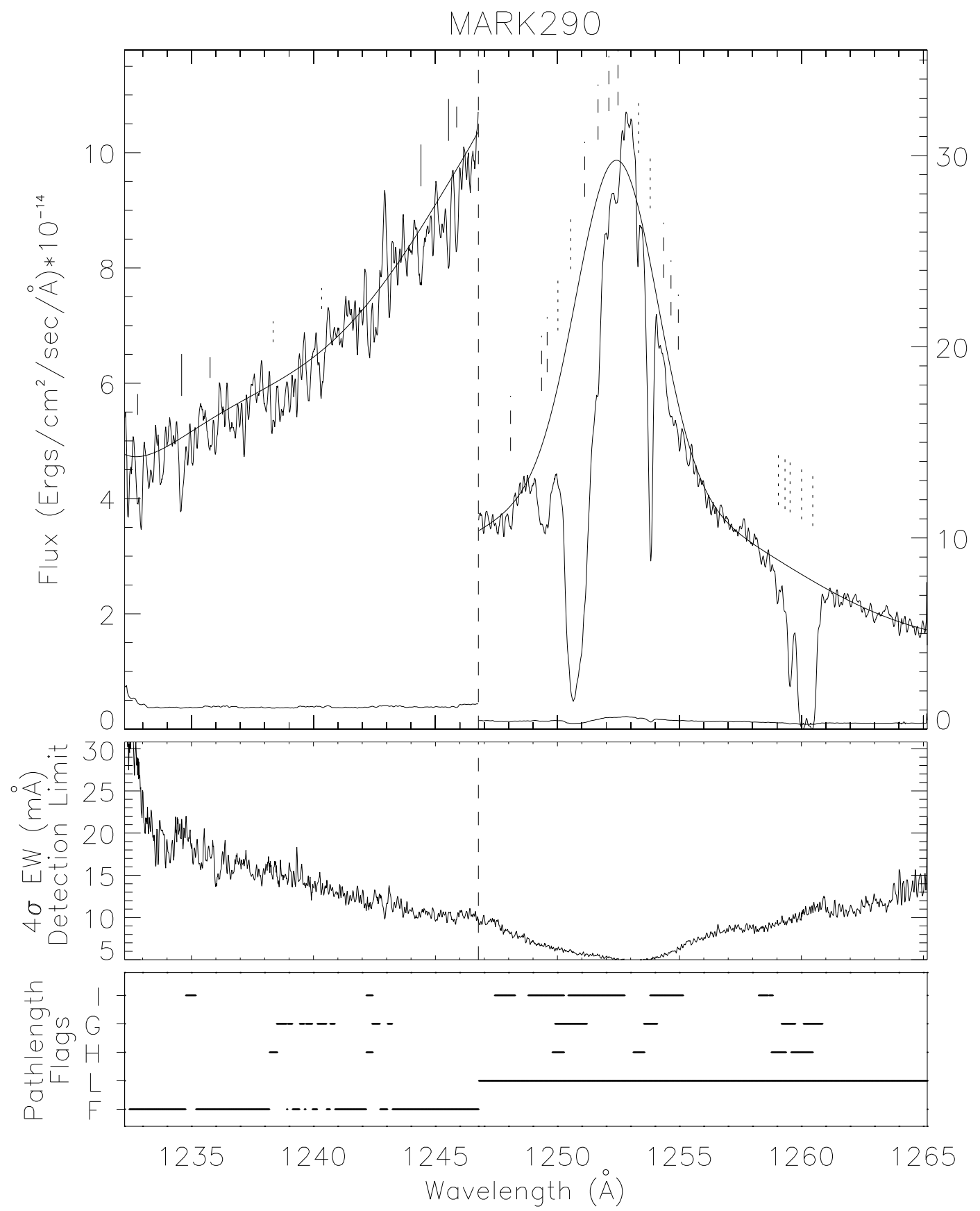

Fig. 12.- GHRS/G160M Markarian 290 spectrum, sensitivity limits, and pathlength flags. 


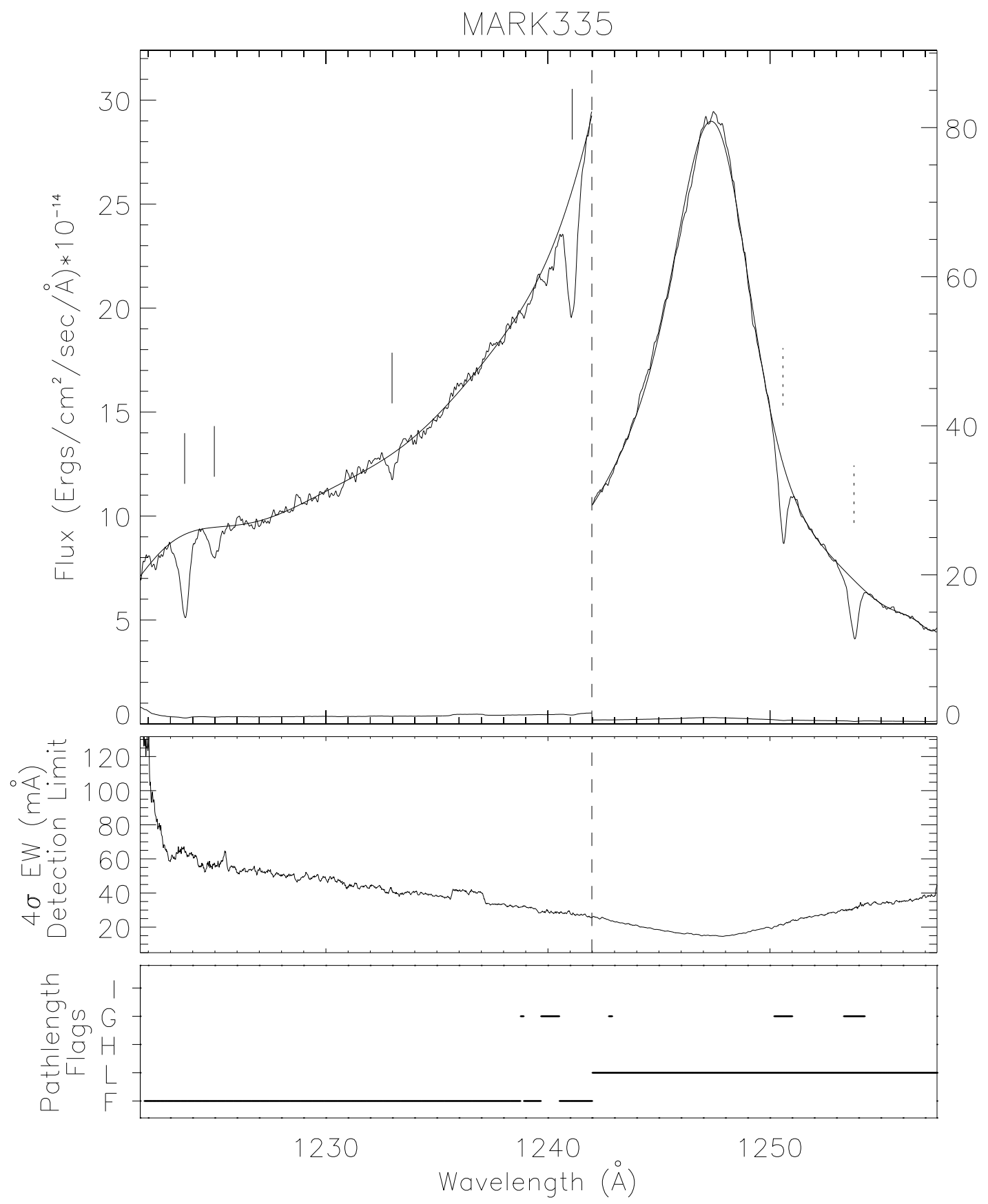

Fig. 13.- GHRS/G160M Markarian 335 spectrum, sensitivity limits, and pathlength flags. 


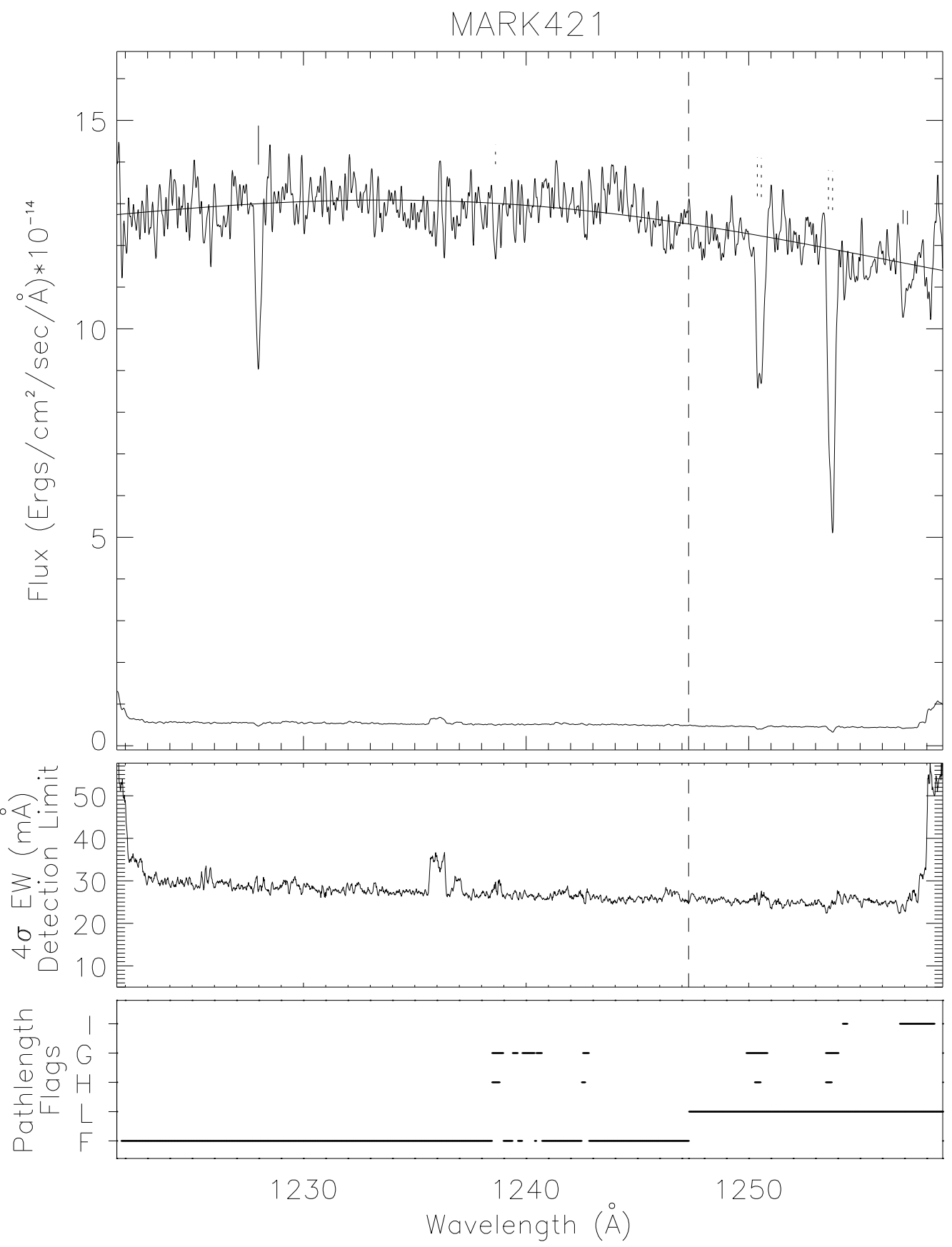

Fig. 14.- GHRS/G160M Markarian 421 spectrum, sensitivity limits, and pathlength flags. 


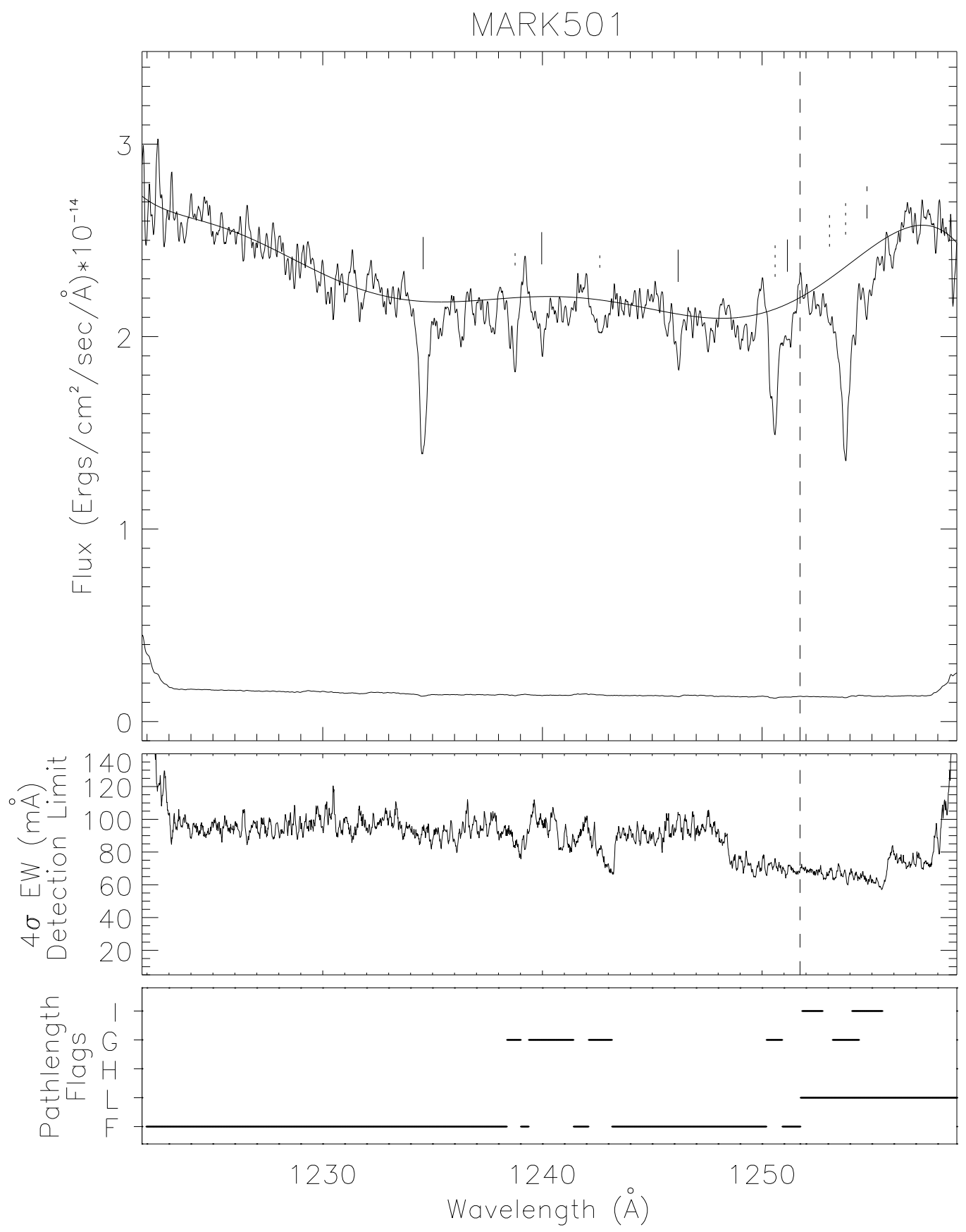

Fig. 15.- GHRS/G160M Markarian 501 spectrum, sensitivity limits, and pathlength flags. 

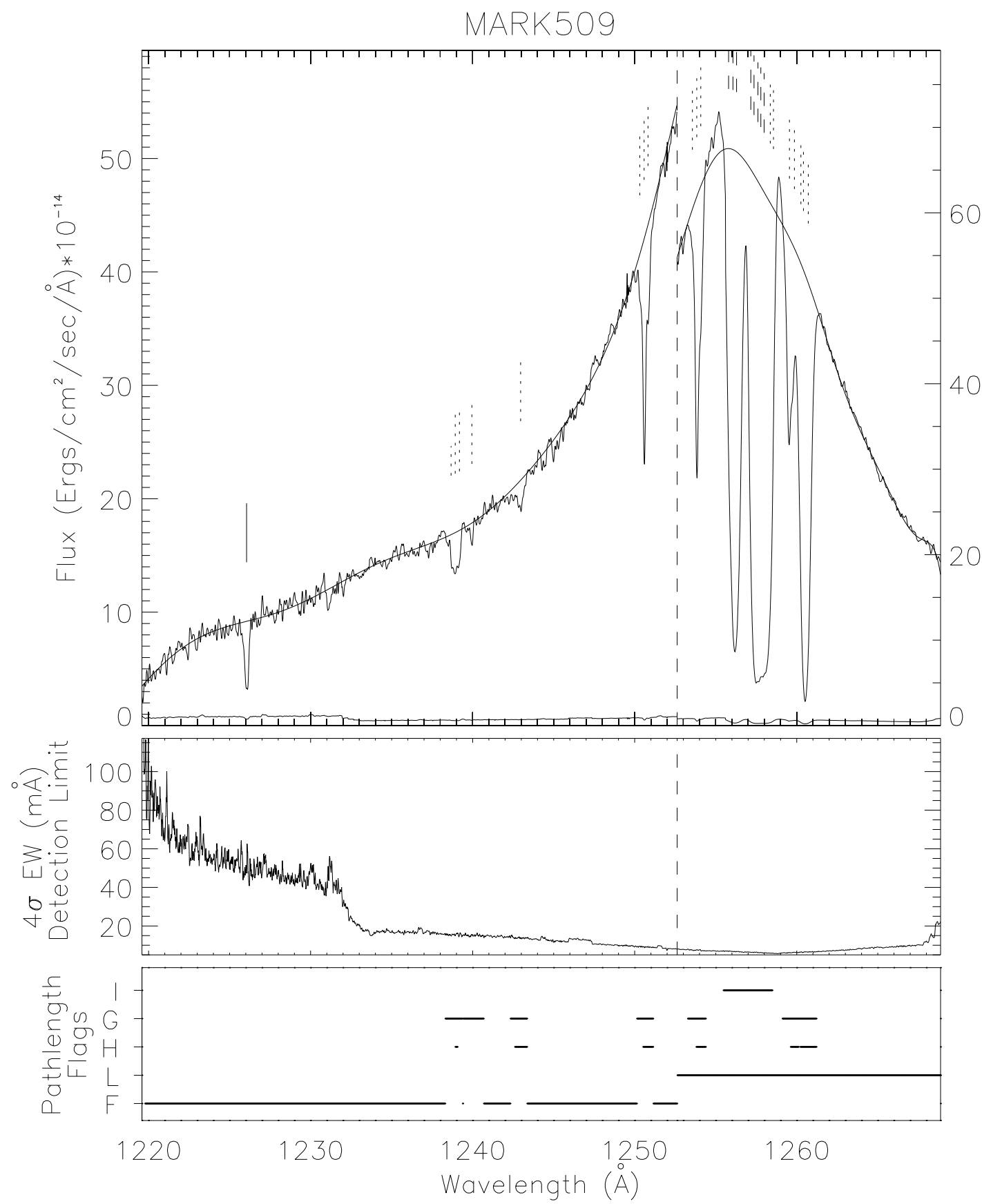

Fig. 16.- GHRS/G160M Markarian 509 spectrum, sensitivity limits, and pathlength flags. 

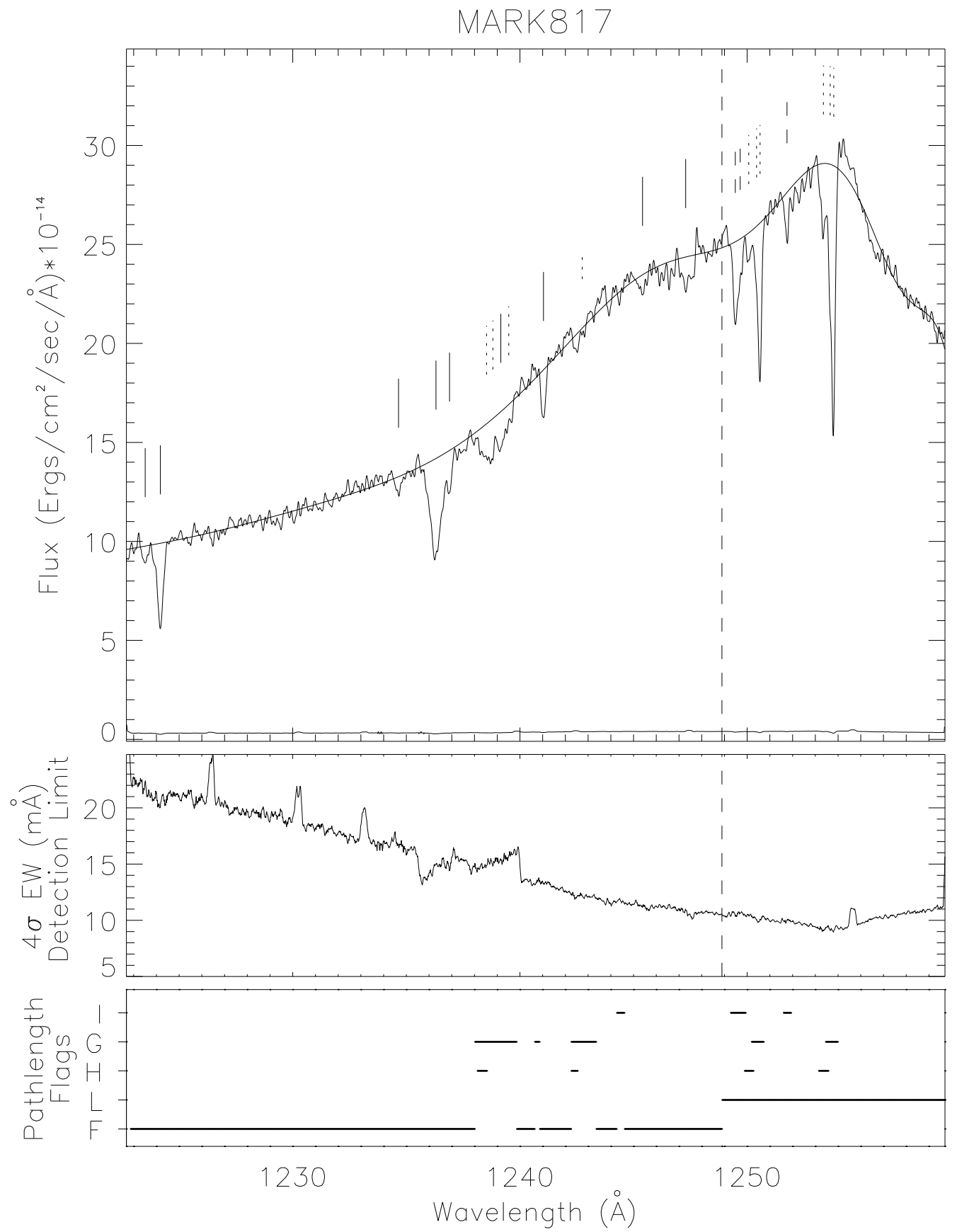

Fig. 17.- GHRS/G160M Markarian 817 spectrum, sensitivity limits, and pathlength flags. 


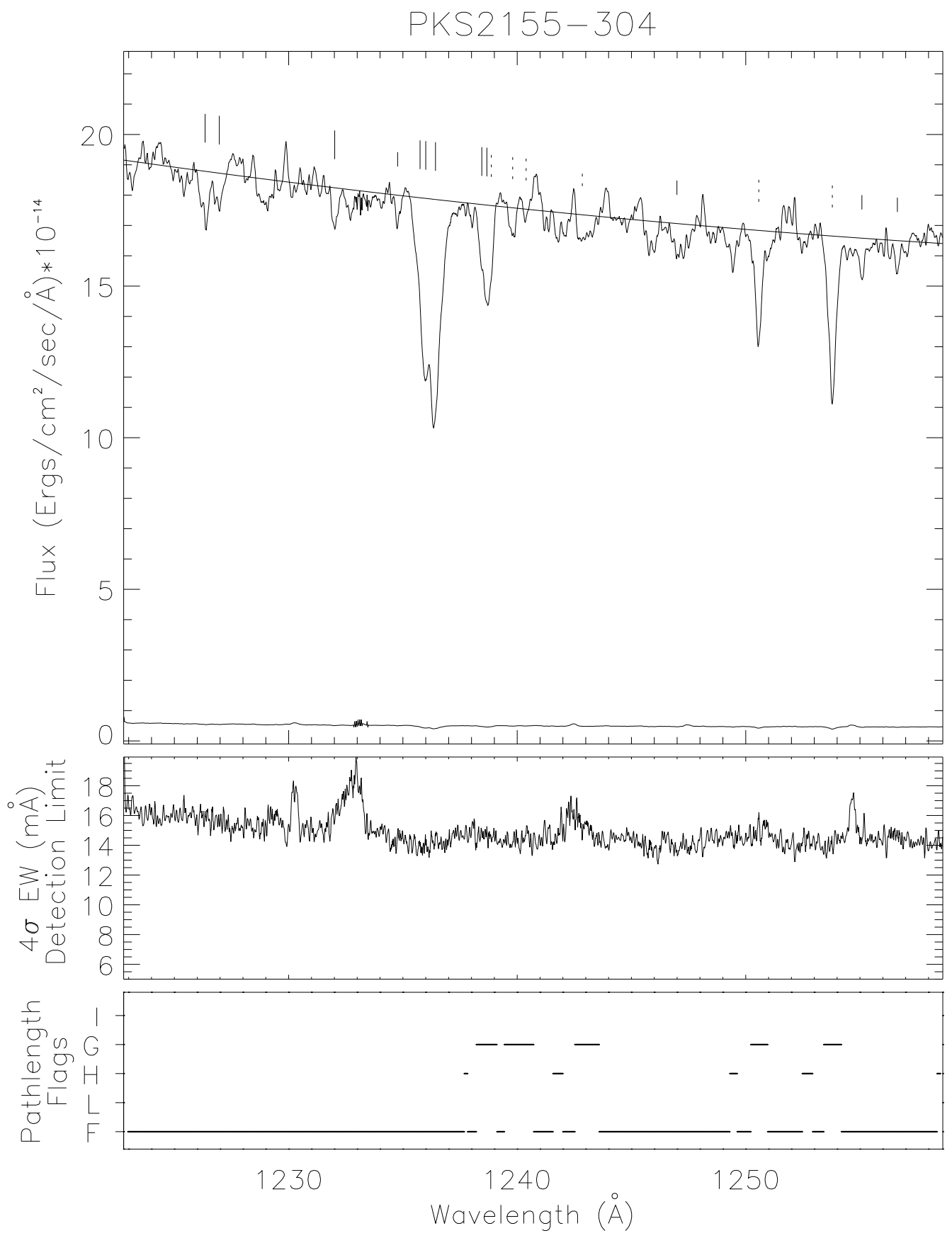

Fig. 18.- Pre-COSTAR GHRS/G160M PKS 2155-304 spectrum, sensitivity limits, and pathlength flags. 


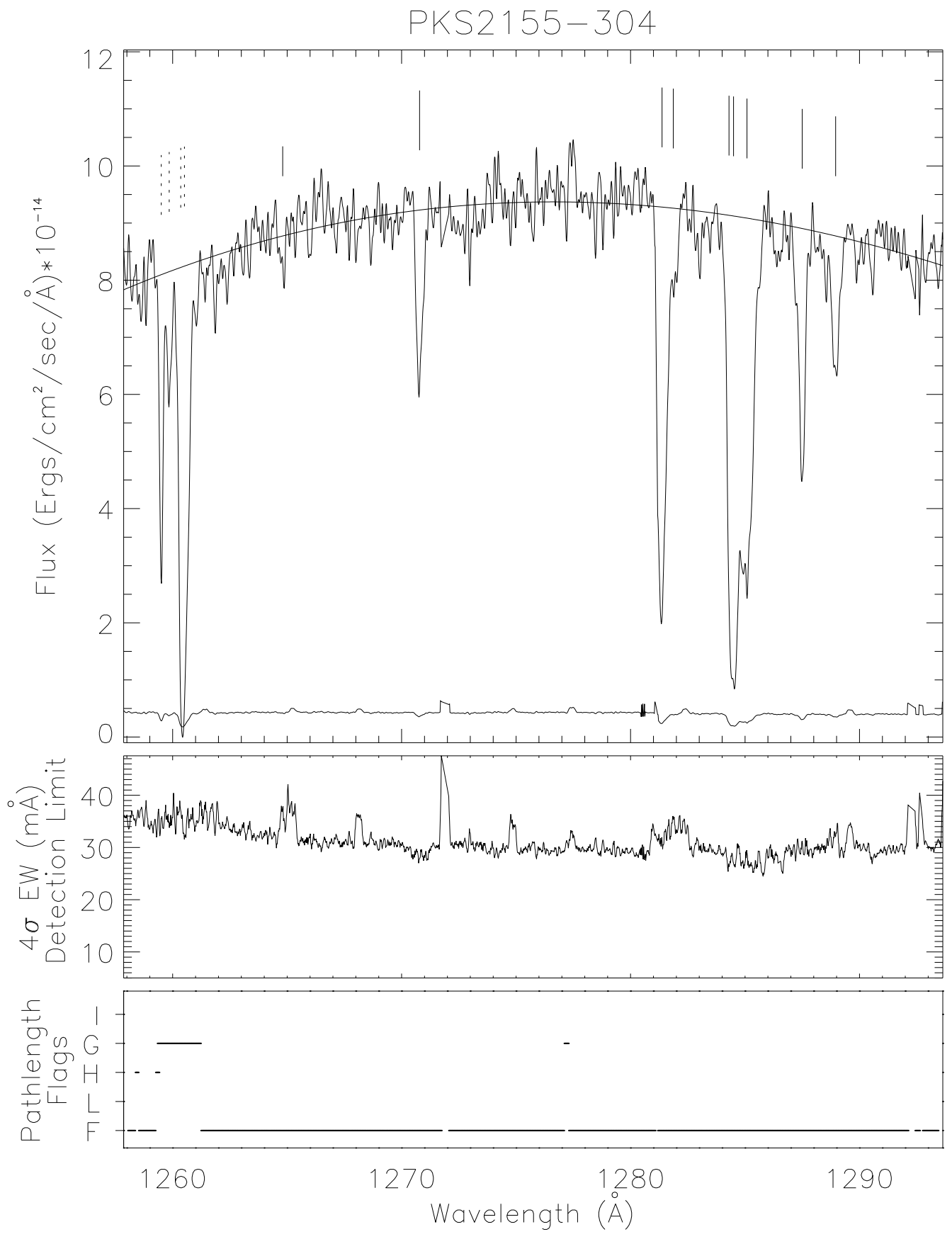

Fig. 19.- Post-COSTAR GHRS/G160M PKS 2155-304 spectrum, sensitivity limits, and pathlength flags. 


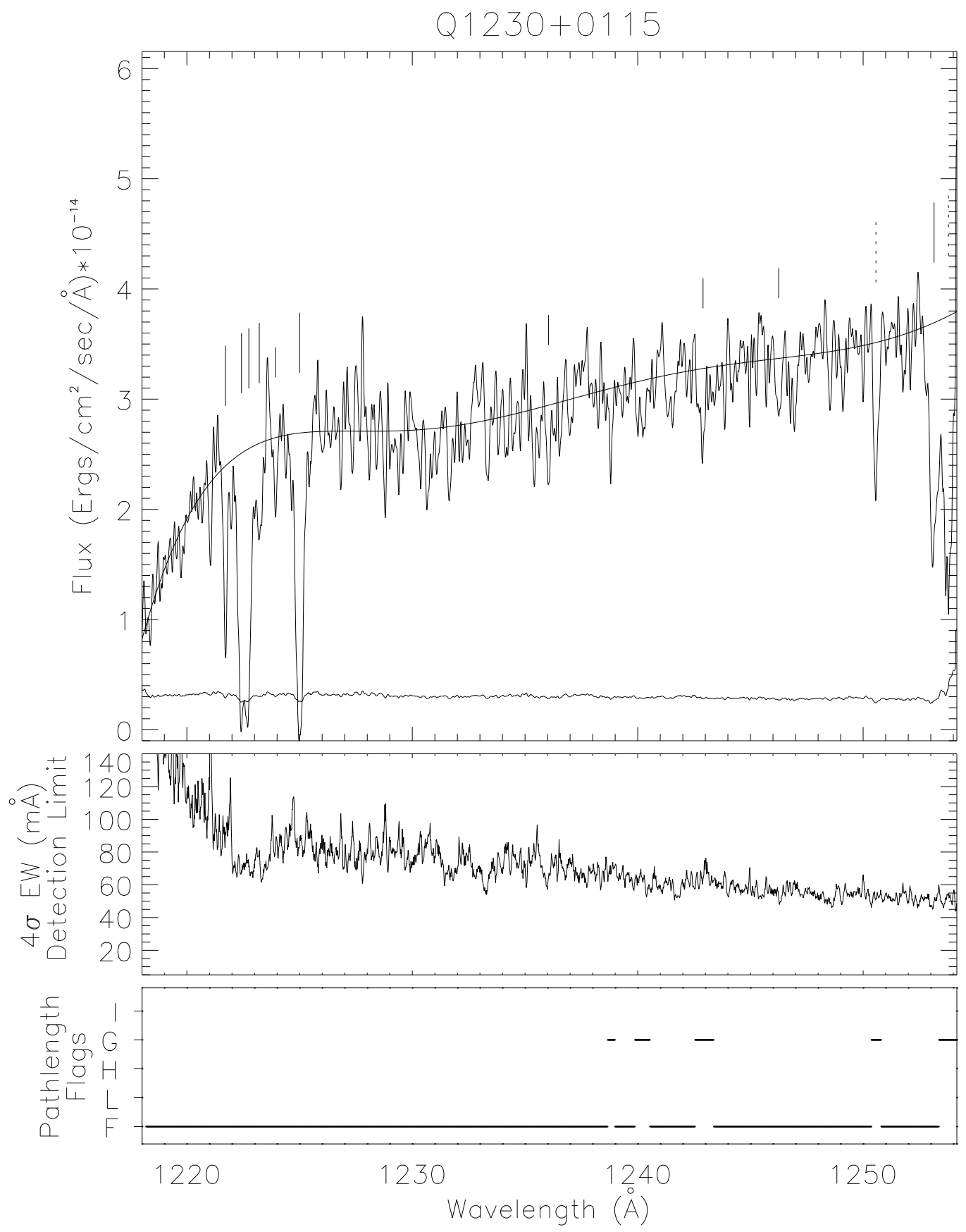

Fig. 20.- GHRS/G160M Q 1230+0115 spectrum, sensitivity limits, and pathlength flags. 
Table 9. HST/GHRS Absorption Features

\begin{tabular}{|c|c|c|c|c|c|c|}
\hline $\begin{array}{c}\text { Wavelength } \\
(\AA)\end{array}$ & $\begin{array}{l}\text { Velocity } \\
\left(\mathrm{km} \mathrm{s}^{-1}\right)\end{array}$ & $\begin{array}{c}b \\
\left(\mathrm{~km} \mathrm{~s}^{-1}\right)\end{array}$ & $\begin{array}{c}\mathcal{W} \\
(\mathrm{m} \AA)\end{array}$ & $\begin{array}{l}\mathrm{SL} \\
(\sigma)\end{array}$ & Id & Alt Id \\
\hline \multicolumn{7}{|c|}{$3 \mathrm{C} 273$} \\
\hline $1219.786 \pm 0.024$ & $1015 \pm 6$ & $69 \pm 5$ & $369 \pm 36$ & 34.8 & $\operatorname{Ly} \alpha$ & $\ldots$ \\
\hline $1222.100 \pm 0.023$ & $1586 \pm 6$ & $72 \pm 4$ & $373 \pm 30$ & 42.0 & $\operatorname{Ly} \alpha$ & $\cdots$ \\
\hline $1224.587 \pm 0.150$ & $2199 \pm 37$ & $57 \pm 55$ & $29 \pm 35$ & 3.5 & $\operatorname{Ly} \alpha$ & $\ldots$ \\
\hline $1224.954 \pm 0.029$ & $2290 \pm 7$ & $54 \pm 33$ & $35 \pm 30$ & 4.2 & $\operatorname{Ly} \alpha$ & $\ldots$ \\
\hline $1234.704 \pm 0.029$ & $4694 \pm 7$ & $69 \pm 61$ & $25 \pm 29$ & 3.5 & $\operatorname{Ly} \alpha$ & $\ldots$ \\
\hline $1238.814 \pm 0.030$ & $-2 \pm 7$ & $87 \pm 8$ & $158 \pm 21$ & 27.2 & g:NV1238.8 & $\cdots$ \\
\hline $1239.991 \pm 0.138$ & $16 \pm 33$ & $67 \pm 47$ & $36 \pm 34$ & 6.1 & g:MgII1239.9 & $\operatorname{Ly} \alpha$ \\
\hline $1242.783 \pm 0.043$ & $-5 \pm 10$ & $76 \pm 15$ & $81 \pm 21$ & 13.5 & $\mathrm{~g}: \mathrm{NV} 1242.8$ & $\ldots$ \\
\hline $1247.593 \pm 0.046$ & $7872 \pm 11$ & $34 \pm 17$ & $33 \pm 18$ & 5.8 & $\operatorname{Ly} \alpha$ & $\cdots$ \\
\hline $1250.589 \pm 0.029$ & $1 \pm 7$ & $60 \pm 8$ & $151 \pm 25$ & 21.4 & g:SII1250.6 & $\cdots$ \\
\hline $1251.485 \pm 0.032$ & $8832 \pm 8$ & $61 \pm 10$ & $114 \pm 25$ & 14.8 & $\operatorname{Ly} \alpha$ & $\cdots$ \\
\hline $1253.780 \pm 0.025$ & $-7 \pm 6$ & $60 \pm 5$ & $207 \pm 23$ & 24.8 & g:SII1253.8 & $\cdots$ \\
\hline $1255.542 \pm 0.069$ & $9833 \pm 17$ & $64 \pm 24$ & $46 \pm 22$ & 5.9 & $\operatorname{Ly} \alpha$ & i:NII1084.0 \\
\hline $1259.483 \pm 0.025$ & $-9 \pm 6$ & $63 \pm 6$ & $235 \pm 28$ & 28.5 & g:SII1259.5 & $\cdots$ \\
\hline $1260.384 \pm 0.036$ & $-18 \pm 9$ & $77 \pm 8$ & $535 \pm 85$ & 64.4 & g:SiII+FeII1260.5 & $\cdots$ \\
\hline $1260.705 \pm 0.029$ & $49 \pm 7$ & $43 \pm 18$ & $57 \pm 64$ & 6.8 & g:SiII+FeII1260.5 & g:CI1260.7 \\
\hline $1265.701 \pm 0.064$ & $12338 \pm 16$ & $33 \pm 26$ & $21 \pm 18$ & 3.3 & $\operatorname{Ly} \alpha$ & z:SiIII1206.5 \\
\hline $1266.724 \pm 0.084$ & $12590 \pm 21$ & $52 \pm 30$ & $24 \pm 18$ & 3.6 & $\operatorname{Ly} \alpha$ & $\cdots$ \\
\hline $1268.969 \pm 0.076$ & $13144 \pm 19$ & $43 \pm 28$ & $18 \pm 15$ & 3.1 & $\operatorname{Ly} \alpha$ & $\ldots$ \\
\hline $1275.243 \pm 0.031$ & $14691 \pm 7$ & $61 \pm 8$ & $140 \pm 25$ & 16.3 & $\operatorname{Ly} \alpha$ & $\ldots$ \\
\hline $1276.442 \pm 0.059$ & $14987 \pm 14$ & $52 \pm 20$ & $46 \pm 22$ & 5.4 & $\operatorname{Ly} \alpha$ & $\cdots$ \\
\hline $1277.474 \pm 0.136$ & $15241 \pm 33$ & $88 \pm 52$ & $52 \pm 40$ & 6.3 & $\operatorname{Ly} \alpha$ & $\cdots$ \\
\hline $1280.267 \pm 0.077$ & $15930 \pm 19$ & $71 \pm 28$ & $64 \pm 33$ & 6.4 & $\operatorname{Ly} \alpha$ & $\cdots$ \\
\hline $1289.767 \pm 0.098$ & $18273 \pm 24$ & $82 \pm 36$ & $47 \pm 28$ & 6.1 & $\operatorname{Ly} \alpha$ & $\cdots$ \\
\hline $1292.851 \pm 0.051$ & $19033 \pm 12$ & $45 \pm 17$ & $47 \pm 22$ & 5.5 & $\operatorname{Ly} \alpha$ & $\cdots$ \\
\hline $1296.591 \pm 0.025$ & $19956 \pm 6$ & $62 \pm 4$ & $297 \pm 25$ & 33.0 & $\operatorname{Ly} \alpha$ & $\ldots$ \\
\hline
\end{tabular}

Arakelian 120 
Table 9-Continued

\begin{tabular}{ccccccc}
\hline \hline $\begin{array}{c}\text { Wavelength } \\
(\AA)\end{array}$ & $\begin{array}{c}\text { Velocity } \\
\left(\mathrm{km} \mathrm{s}^{-1}\right)\end{array}$ & $\begin{array}{c}b \\
\left(\mathrm{~km} \mathrm{~s}^{-1}\right)\end{array}$ & $\begin{array}{c}\mathcal{W} \\
(\mathrm{m} \AA)\end{array}$ & $\begin{array}{c}\text { SL } \\
(\sigma)\end{array}$ & Id & Alt Id \\
\hline $1223.088 \pm 0.039$ & $1829 \pm 10$ & $25 \pm 17$ & $64 \pm 48$ & 3.7 & Ly $\alpha$ & $\ldots$ \\
$1232.052 \pm 0.034$ & $4040 \pm 8$ & $32 \pm 11$ & $48 \pm 18$ & 5.5 & Ly $\alpha$ & $\ldots$ \\
$1238.789 \pm 0.029$ & $-8 \pm 7$ & $28 \pm 19$ & $29 \pm 20$ & 3.9 & g:NV1238.8 & Ly $\alpha$ \\
$1239.934 \pm 0.023$ & $2 \pm 6$ & $19 \pm 5$ & $60 \pm 12$ & 8.5 & g:MgII1239.9 & Ly $\alpha$ \\
$1240.427 \pm 0.028$ & $8 \pm 7$ & $20 \pm 8$ & $34 \pm 12$ & 5.0 & g:MgII1240.4 & $\ldots$ \\
$1242.972 \pm 0.028$ & $6733 \pm 7$ & $33 \pm 8$ & $53 \pm 13$ & 9.2 & Ly $\alpha$ & g:NV1242.8 \\
$1247.267 \pm 0.104$ & $7792 \pm 26$ & $34 \pm 32$ & $19 \pm 22$ & 3.9 & Ly $\alpha$ & $\ldots$ \\
$1247.570 \pm 0.087$ & $7867 \pm 21$ & $34 \pm 35$ & $20 \pm 25$ & 4.3 & Ly $\alpha$ & $\ldots$ \\
$1247.948 \pm 0.023$ & $7960 \pm 5$ & $27 \pm 4$ & $147 \pm 22$ & 31.4 & Ly $\alpha$ & $\ldots$ \\
$1248.192 \pm 0.027$ & $8020 \pm 7$ & $23 \pm 6$ & $65 \pm 17$ & 14.1 & Ly $\alpha$ & $\ldots$ \\
$1250.602 \pm 0.023$ & $4 \pm 5$ & $31 \pm 3$ & $224 \pm 8$ & 57.2 & g:SII1250.6 & $\ldots$ \\
$1252.135 \pm 0.034$ & $-907 \pm 8$ & $28 \pm 11$ & $18 \pm 7$ & 5.1 & $\mathrm{i}:$ Ly $\alpha$ & $\ldots$ \\
$1253.835 \pm 0.023$ & $6 \pm 5$ & $34 \pm 3$ & $258 \pm 8$ & 80.2 & $\mathrm{~g}:$ SII1253.8 & $\mathrm{i}:$ Ly $\alpha$ \\
\hline
\end{tabular}

ESO 141-G55

\begin{tabular}{|c|c|c|c|c|c|c|}
\hline $1238.815 \pm 0.034$ & $-1 \pm 8$ & $43 \pm 15$ & $75 \pm 29$ & 10.9 & g:NV1238.8 & $\cdots$ \\
\hline $1239.899 \pm 0.028$ & $-6 \pm 7$ & $21 \pm 9$ & $34 \pm 12$ & 5.0 & g:MgII1239.9 & .. \\
\hline $1240.379 \pm 0.026$ & $-4 \pm 6$ & $19 \pm 7$ & $36 \pm 11$ & 5.6 & g:MgII1240.4 & $\cdots$ \\
\hline $1242.772 \pm 0.043$ & $-8 \pm 10$ & $44 \pm 15$ & $46 \pm 19$ & 7.2 & g:NV1242.8 & $\cdots$ \\
\hline $1249.932 \pm 0.036$ & $8449 \pm 9$ & $17 \pm 15$ & $12 \pm 8$ & 3.0 & $\operatorname{Ly} \alpha$ & $\cdots$ \\
\hline $1250.578 \pm 0.023$ & $-2 \pm 5$ & $33 \pm 3$ & $146 \pm 9$ & 35.6 & g:SII1250.6 & $\cdots$ \\
\hline $1252.483 \pm 0.041$ & $9078 \pm 10$ & $23 \pm 16$ & $12 \pm 7$ & 3.1 & $\operatorname{Ly} \alpha$ & $\cdots$ \\
\hline $1253.444 \pm 0.038$ & $-85 \pm 9$ & $27 \pm 13$ & $18 \pm 9$ & 4.7 & h:SII1253.8 & $\cdots$ \\
\hline $1253.815 \pm 0.023$ & $1 \pm 5$ & $37 \pm 3$ & $199 \pm 10$ & 50.4 & g:SII1253.8 & $\cdots$ \\
\hline $1257.716 \pm 0.029$ & $-733 \pm 7$ & $53 \pm 38$ & $18 \pm 20$ & 5.7 & $\mathrm{i}: \operatorname{Ly} \alpha$ & $\cdots$ \\
\hline $1258.093 \pm 0.023$ & $-643 \pm 5$ & $46 \pm 3$ & $353 \pm 11$ & 116.3 & $\mathrm{i}: \operatorname{Ly} \alpha$ & $\cdots$ \\
\hline $1259.304 \pm 0.029$ & $-47 \pm 7$ & $48 \pm 23$ & $23 \pm 14$ & 5.8 & h:SII1259.5 & $\cdots$ \\
\hline $1259.521 \pm 0.029$ & $1 \pm 7$ & $38 \pm 3$ & $217 \pm 11$ & 55.4 & g:SII1259.5 & $\cdots$ \\
\hline $1260.204 \pm 0.029$ & $-70 \pm 7$ & $44 \pm 6$ & $83 \pm 73$ & 21.1 & h:SiII+FeII1260.5 & $\cdots$ \\
\hline $1260.429 \pm 0.049$ & $-7 \pm 12$ & $59 \pm 12$ & $408 \pm 144$ & 102.8 & g:SiII+FeII1260.5 & $\cdots$ \\
\hline $1260.650 \pm 0.026$ & $36 \pm 6$ & $28 \pm 8$ & $100 \pm 71$ & 24.8 & g:SiII+FeII1260.5 & g:CI1260.7 \\
\hline
\end{tabular}


Table 9 -Continued

\begin{tabular}{|c|c|c|c|c|c|c|}
\hline $\begin{array}{c}\text { Wavelength } \\
(\AA)\end{array}$ & $\begin{array}{l}\text { Velocity } \\
\left(\mathrm{km} \mathrm{s}^{-1}\right)\end{array}$ & $\begin{array}{c}b \\
\left(\mathrm{~km} \mathrm{~s}^{-1}\right)\end{array}$ & $\begin{array}{c}\mathcal{W} \\
(\mathrm{m} \AA)\end{array}$ & $\begin{array}{l}\text { SL } \\
(\sigma)\end{array}$ & Id & Alt Id \\
\hline \multicolumn{7}{|c|}{ Fairall 9} \\
\hline $1238.818 \pm 0.026$ & $-1 \pm 6$ & $32 \pm 6$ & $48 \pm 11$ & 9.7 & $\mathrm{~g}: \mathrm{NV} 1238.8$ & $\ldots$ \\
\hline $1239.855 \pm 0.029$ & $-17 \pm 7$ & $17 \pm 10$ & $21 \pm 9$ & 3.9 & g:MgII1239.9 & h:NV1238.8 \\
\hline $1240.402 \pm 0.036$ & $2 \pm 9$ & $19 \pm 13$ & $15 \pm 8$ & 3.0 & g:MgII1240.4 & $\cdots$ \\
\hline $1240.988 \pm 0.038$ & $6244 \pm 9$ & $35 \pm 13$ & $22 \pm 9$ & 5.3 & $\operatorname{Ly} \alpha$ & h:MgII1239.9 \\
\hline $1244.462 \pm 0.034$ & $7100 \pm 8$ & $39 \pm 11$ & $32 \pm 10$ & 8.2 & $\operatorname{Ly} \alpha$ & $\ldots$ \\
\hline $1250.569 \pm 0.023$ & $-4 \pm 5$ & $20 \pm 4$ & $80 \pm 6$ & 22.4 & g:SII1250.6 & $\ldots$ \\
\hline $1251.367 \pm 0.025$ & $188 \pm 6$ & $32 \pm 5$ & $41 \pm 7$ & 11.3 & h:SII1250.6 & $\operatorname{Ly} \alpha$ \\
\hline $1253.787 \pm 0.023$ & $-6 \pm 5$ & $21 \pm 4$ & $106 \pm 7$ & 32.2 & g:SII1253.8 & $\ldots$ \\
\hline $1254.139 \pm 0.024$ & $9487 \pm 6$ & $43 \pm 6$ & $84 \pm 13$ & 26.5 & $\operatorname{Ly} \alpha$ & $\cdots$ \\
\hline $1254.572 \pm 0.023$ & $182 \pm 5$ & $27 \pm 4$ & $56 \pm 7$ & 17.6 & h:SII1253.8 & $\cdots$ \\
\hline $1259.483 \pm 0.023$ & $-9 \pm 5$ & $24 \pm 3$ & $130 \pm 7$ & 36.7 & g:SII1259.5 & $\ldots$ \\
\hline $1260.338 \pm 0.029$ & $199 \pm 7$ & $15 \pm 5$ & $34 \pm 9$ & 8.9 & h:SII1259.5 & $\mathrm{g}: \mathrm{SiII}+\mathrm{FeII} 1260.5$ \\
\hline $1260.468 \pm 0.029$ & $2 \pm 7$ & $61 \pm 3$ & $406 \pm 19$ & 106.6 & $\mathrm{~g}: \mathrm{SiII}+\mathrm{FeII} 1260.5$ & h:SII1259.5 \\
\hline $1261.039 \pm 0.035$ & $128 \pm 8$ & $54 \pm 6$ & $379 \pm 75$ & 105.2 & h:SiII+FeII1260.5 & $\mathrm{g}: \mathrm{CI} 1260.7 ?$ \\
\hline $1261.300 \pm 0.028$ & $190 \pm 7$ & $36 \pm 4$ & $180 \pm 62$ & 51.3 & h:SiII+FeII1260.5 & $\cdots$ \\
\hline $1262.864 \pm 0.029$ & $11638 \pm 7$ & $28 \pm 13$ & $16 \pm 8$ & 5.2 & $\operatorname{Ly} \alpha$ & $\ldots$ \\
\hline $1263.998 \pm 0.041$ & $11918 \pm 10$ & $42 \pm 15$ & $22 \pm 9$ & 7.0 & $\operatorname{Ly} \alpha$ & $\cdots$ \\
\hline $1264.684 \pm 0.073$ & $12087 \pm 18$ & $44 \pm 33$ & $30 \pm 28$ & 10.2 & $\operatorname{Ly} \alpha$ & $\cdots$ \\
\hline $1265.104 \pm 0.026$ & $12191 \pm 6$ & $23 \pm 7$ & $28 \pm 7$ & 9.5 & $\operatorname{Ly} \alpha$ & $\ldots$ \\
\hline $1265.407 \pm 0.029$ & $12265 \pm 7$ & $19 \pm 18$ & $11 \pm 8$ & 3.8 & $\operatorname{Ly} \alpha$ & $\cdots$ \\
\hline $1265.970 \pm 0.117$ & $12404 \pm 29$ & $32 \pm 23$ & $19 \pm 23$ & 7.0 & $\operatorname{Ly} \alpha$ & $\ldots$ \\
\hline $1268.850 \pm 0.029$ & $-688 \pm 7$ & $81 \pm 22$ & $32 \pm 12$ & 11.2 & $\mathrm{i}: \operatorname{Ly} \alpha$ & $\cdots$ \\
\hline $1269.351 \pm 0.029$ & $-570 \pm 7$ & $46 \pm 19$ & $16 \pm 9$ & 5.4 & $\mathrm{i}: \operatorname{Ly} \alpha$ & $\cdots$ \\
\hline $1269.731 \pm 0.029$ & $-480 \pm 7$ & $99 \pm 3$ & $74 \pm 7$ & 26.7 & $\mathrm{i}: \operatorname{Ly} \alpha$ & $\ldots$ \\
\hline $1270.288 \pm 0.032$ & $-349 \pm 8$ & $89 \pm 12$ & $93 \pm 16$ & 32.2 & $\mathrm{i}: \operatorname{Ly} \alpha$ & $\cdots$ \\
\hline $1270.746 \pm 0.027$ & $-241 \pm 6$ & $11 \pm 11$ & $11 \pm 6$ & 4.2 & $\mathrm{i}: \operatorname{Ly} \alpha$ & $\ldots$ \\
\hline $1270.955 \pm 0.029$ & $-191 \pm 7$ & $42 \pm 5$ & $55 \pm 9$ & 20.3 & $\mathrm{i}: \operatorname{Ly} \alpha$ & $\cdots$ \\
\hline
\end{tabular}


Table 9-Continued

\begin{tabular}{|c|c|c|c|c|c|c|}
\hline $\begin{array}{c}\text { Wavelength } \\
(\AA)\end{array}$ & $\begin{array}{l}\text { Velocity } \\
\left(\mathrm{km} \mathrm{s}^{-1}\right)\end{array}$ & $\begin{array}{c}b \\
\left(\mathrm{~km} \mathrm{~s}^{-1}\right)\end{array}$ & $\begin{array}{c}\mathcal{W} \\
(\mathrm{m} \AA)\end{array}$ & $\begin{array}{l}\mathrm{SL} \\
(\sigma)\end{array}$ & $\mathrm{Id}$ & Alt Id \\
\hline $1238.014 \pm 0.036$ & $5510 \pm 9$ & $21 \pm 14$ & $23 \pm 13$ & 3.3 & $\operatorname{Ly} \alpha$ & $\cdots$ \\
\hline $1238.608 \pm 0.029$ & $-47 \pm 7$ & $38 \pm 22$ & $42 \pm 27$ & 5.9 & h:NV1238.8 & $\cdots$ \\
\hline $1238.826 \pm 0.029$ & $1 \pm 7$ & $28 \pm 8$ & $93 \pm 29$ & 13.1 & g:NV1238.8 & $\operatorname{Ly} \alpha$ \\
\hline $1238.938 \pm 0.029$ & $28 \pm 7$ & $10 \pm 10$ & $34 \pm 15$ & 4.7 & $\mathrm{~g}: \mathrm{NV} 1238.8$ & h:MgII1239.9? \\
\hline $1239.938 \pm 0.029$ & $3 \pm 7$ & $34 \pm 9$ & $59 \pm 18$ & 8.5 & g:MgII1239.9 & h:MgII1240.4 \\
\hline $1240.569 \pm 0.036$ & $6140 \pm 9$ & $14 \pm 16$ & $24 \pm 16$ & 3.4 & $\operatorname{Ly} \alpha$ & g:MgII1240.4 \\
\hline $1242.616 \pm 0.029$ & $-44 \pm 7$ & $42 \pm 20$ & $56 \pm 30$ & 8.2 & h:NV1242.8 & g:NV1242.8 \\
\hline $1242.880 \pm 0.025$ & $18 \pm 6$ & $23 \pm 5$ & $65 \pm 17$ & 9.5 & $\mathrm{~g}: \mathrm{NV} 1242.8$ & $\mathrm{z}: \operatorname{Ly} \beta$ \\
\hline $1244.611 \pm 0.023$ & $0.21340 \pm 0.00002$ & $46 \pm 4$ & $153 \pm 18$ & 25.4 & $\mathrm{z}: \operatorname{Ly} \beta$ & $\ldots$ \\
\hline $1244.966 \pm 0.031$ & $7225 \pm 8$ & $15 \pm 12$ & $25 \pm 13$ & 3.5 & $\operatorname{Ly} \alpha$ & $\cdots$ \\
\hline $1245.440 \pm 0.023$ & $7342 \pm 5$ & $47 \pm 3$ & $298 \pm 20$ & 42.4 & $\operatorname{Ly} \alpha$ & z:NIII989.8 \\
\hline $1246.301 \pm 0.036$ & $7554 \pm 9$ & $41 \pm 14$ & $50 \pm 24$ & 7.1 & $\operatorname{Ly} \alpha$ & $\cdots$ \\
\hline $1247.362 \pm 0.029$ & $7815 \pm 7$ & $36 \pm 34$ & $27 \pm 29$ & 3.7 & $\operatorname{Ly} \alpha$ & z:Ly $\beta$ ? \\
\hline $1247.583 \pm 0.029$ & $7870 \pm 7$ & $19 \pm 9$ & $40 \pm 17$ & 5.4 & $\operatorname{Ly} \alpha$ & $\ldots$ \\
\hline $1247.937 \pm 0.033$ & $7957 \pm 8$ & $33 \pm 10$ & $68 \pm 38$ & 9.4 & $\operatorname{Ly} \alpha$ & $\cdots$ \\
\hline $1250.314 \pm 0.029$ & $-65 \pm 7$ & $26 \pm 13$ & $30 \pm 17$ & 4.4 & h:SII1250.6 & z:OVI1031.9 \\
\hline $1250.577 \pm 0.023$ & $-2 \pm 5$ & $39 \pm 3$ & $190 \pm 19$ & 28.0 & g:SII1250.6 & z:OVI1031.9 \\
\hline $1252.177 \pm 0.032$ & $0.21344 \pm 0.00003$ & $34 \pm 11$ & $51 \pm 17$ & 9.3 & z:OVI1031.9 & $\cdots$ \\
\hline $1252.477 \pm 0.042$ & $9077 \pm 10$ & $21 \pm 15$ & $23 \pm 15$ & 3.6 & $\operatorname{Ly} \alpha$ & $\cdots$ \\
\hline $1253.314 \pm 0.029$ & $-119 \pm 7$ & $17 \pm 10$ & $31 \pm 13$ & 5.0 & h:SII1253.8 & g:SII1253.8? \\
\hline $1253.792 \pm 0.023$ & $-4 \pm 5$ & $47 \pm 3$ & $263 \pm 22$ & 41.6 & g:SII1253.8 & z:NIII989.8 \\
\hline $1254.874 \pm 0.099$ & $9668 \pm 24$ & $24 \pm 31$ & $21 \pm 25$ & 3.6 & $\operatorname{Ly} \alpha$ & z:OVI1031.9? \\
\hline $1256.309 \pm 0.029$ & $0.22480 \pm 0.00003$ & $47 \pm 3$ & $199 \pm 19$ & 38.4 & $\mathrm{z}: \operatorname{Ly} \beta$ & $\cdots$ \\
\hline $1256.635 \pm 0.023$ & $0.22512 \pm 0.00002$ & $57 \pm 3$ & $310 \pm 21$ & 58.9 & $\mathrm{z}: \operatorname{Ly} \beta$ & $\cdots$ \\
\hline $1257.659 \pm 0.029$ & $0.22612 \pm 0.00002$ & $31 \pm 22$ & $19 \pm 14$ & 3.8 & $\mathrm{z}: \operatorname{Ly} \beta$ & $\ldots$ \\
\hline $1259.157 \pm 0.029$ & $-86 \pm 7$ & $59 \pm 10$ & $99 \pm 24$ & 16.2 & h:SII1259.5 & $\mathrm{g}: \mathrm{SII} 1259.5 ?$ \\
\hline $1259.532 \pm 0.029$ & $3 \pm 7$ & $46 \pm 3$ & $275 \pm 24$ & 45.6 & g:SII1259.5 & $\cdots$ \\
\hline $1260.052 \pm 0.029$ & $-107 \pm 7$ & $59 \pm 4$ & $440 \pm 35$ & 74.4 & h:SiII+FeII1260.5 & $\ldots$ \\
\hline $1260.298 \pm 0.036$ & $-48 \pm 9$ & $23 \pm 22$ & $39 \pm 47$ & 6.6 & $\mathrm{~g}: \mathrm{SiII}+\mathrm{FeII} 1260.5$ & $\ldots$ \\
\hline $1260.514 \pm 0.047$ & $3 \pm 6$ & $57 \pm 9$ & $407 \pm 102$ & 70.0 & $\mathrm{~g}: \mathrm{SiII}+\mathrm{FeII} 1260.5$ & g:CI1260.7? \\
\hline $1261.262 \pm 0.029$ & $0.29688 \pm 0.00003$ & $67 \pm 4$ & $252 \pm 19$ & 57.8 & $\mathrm{z}: \mathrm{Ly} \gamma$ & $\cdots$ \\
\hline
\end{tabular}


Table 9 - Continued

\begin{tabular}{|c|c|c|c|c|c|c|}
\hline $\begin{array}{c}\text { Wavelength } \\
(\AA)\end{array}$ & $\begin{array}{l}\text { Velocity } \\
\left(\mathrm{km} \mathrm{s}^{-1}\right)\end{array}$ & $\begin{array}{c}b \\
\left(\mathrm{~km} \mathrm{~s}^{-1}\right)\end{array}$ & $\begin{array}{c}\mathcal{W} \\
(\mathrm{m} \AA)\end{array}$ & $\begin{array}{l}\mathrm{SL} \\
(\sigma)\end{array}$ & Id & Alt Id \\
\hline $1264.194 \pm 0.029$ & $0.22511 \pm 0.00003$ & $39 \pm 5$ & $113 \pm 20$ & 24.8 & z:OVI1031.9 & $\cdots$ \\
\hline $1264.413 \pm 0.037$ & $0.22533 \pm 0.00003$ & $43 \pm 11$ & $77 \pm 24$ & 16.7 & z:OVI1031.9 & $\ldots$ \\
\hline $1265.394 \pm 0.029$ & $0.22624 \pm 0.00002$ & $18 \pm 18$ & $16 \pm 12$ & 3.5 & z:OVI1031.9 & $\operatorname{Ly} \alpha$ \\
\hline $1265.683 \pm 0.025$ & $12334 \pm 6$ & $28 \pm 6$ & $64 \pm 15$ & 11.8 & $\operatorname{Ly} \alpha$ & $\cdots$ \\
\hline $1267.061 \pm 0.023$ & $0.29689 \pm 0.00002$ & $58 \pm 3$ & $259 \pm 13$ & 59.2 & z:CIII977.0 & $\cdots$ \\
\hline \multicolumn{7}{|c|}{ I ZW 1} \\
\hline $1250.545 \pm 0.037$ & $-9 \pm 9$ & $40 \pm 11$ & $91 \pm 30$ & 6.8 & g:SII1250.6 & $\ldots$ \\
\hline $1253.808 \pm 0.028$ & $-1 \pm 7$ & $34 \pm 7$ & $115 \pm 26$ & 9.1 & g:SII1253.8 & $\cdots$ \\
\hline \multicolumn{7}{|c|}{ Markarian 279} \\
\hline $1236.942 \pm 0.030$ & $5246 \pm 7$ & $27 \pm 9$ & $30 \pm 10$ & 5.6 & $\operatorname{Ly} \alpha$ & $\ldots$ \\
\hline $1237.915 \pm 0.029$ & $5486 \pm 7$ & $30 \pm 29$ & $17 \pm 18$ & 3.5 & $\operatorname{Ly} \alpha$ & $\cdots$ \\
\hline $1238.260 \pm 0.037$ & $-136 \pm 9$ & $20 \pm 18$ & $22 \pm 16$ & 4.3 & h:NV1238.8 & $\operatorname{Ly} \alpha$ \\
\hline $1238.502 \pm 0.047$ & $5631 \pm 12$ & $21 \pm 23$ & $18 \pm 18$ & 3.7 & $\operatorname{Ly} \alpha$ & $\mathrm{g}: \mathrm{NV} 1238.8 ?$ \\
\hline $1238.811 \pm 0.035$ & $-2 \pm 8$ & $39 \pm 16$ & $50 \pm 24$ & 10.3 & g:NV1238.8 & $\cdots$ \\
\hline $1241.509 \pm 0.029$ & $6372 \pm 7$ & $18 \pm 4$ & $58 \pm 7$ & 14.2 & $\operatorname{Ly} \alpha$ & $\ldots$ \\
\hline $1241.805 \pm 0.023$ & $6445 \pm 6$ & $21 \pm 4$ & $40 \pm 7$ & 10.4 & $\operatorname{Ly} \alpha$ & $\ldots$ \\
\hline $1243.753 \pm 0.023$ & $6925 \pm 5$ & $26 \pm 3$ & $65 \pm 8$ & 16.8 & $\operatorname{Ly} \alpha$ & i:SiIII1206.5? \\
\hline $1247.216 \pm 0.024$ & $7779 \pm 6$ & $28 \pm 5$ & $48 \pm 9$ & 15.1 & $\operatorname{Ly} \alpha$ & $\cdots$ \\
\hline $1247.533 \pm 0.042$ & $7858 \pm 10$ & $34 \pm 14$ & $21 \pm 10$ & 6.7 & $\operatorname{Ly} \alpha$ & $\ldots$ \\
\hline $1248.410 \pm 0.029$ & $-1070 \pm 7$ & $18 \pm 12$ & $11 \pm 6$ & 3.6 & $\mathrm{i}: \operatorname{Ly} \alpha$ & $\ldots$ \\
\hline $1249.023 \pm 0.036$ & $-923 \pm 9$ & $43 \pm 12$ & $22 \pm 8$ & 7.5 & $\mathrm{i}: \operatorname{Ly} \alpha$ & $\cdots$ \\
\hline $1249.933 \pm 0.034$ & $-160 \pm 8$ & $23 \pm 14$ & $12 \pm 8$ & 4.0 & h:SII1250.6 & $\ldots$ \\
\hline $1250.576 \pm 0.029$ & $-2 \pm 7$ & $62 \pm 3$ & $282 \pm 17$ & 99.5 & g:SII1250.6 & $\cdots$ \\
\hline $1251.056 \pm 0.025$ & $-436 \pm 6$ & $99 \pm 3$ & $646 \pm 18$ & 240.5 & $\mathrm{i}: \operatorname{Ly} \alpha$ & $\ldots$ \\
\hline $1251.261 \pm 0.029$ & $-387 \pm 7$ & $19 \pm 10$ & $17 \pm 10$ & 6.6 & $\mathrm{i}: \operatorname{Ly} \alpha$ & $\cdots$ \\
\hline $1251.694 \pm 0.023$ & $-284 \pm 5$ & $71 \pm 3$ & $322 \pm 14$ & 123.9 & $\mathrm{i}: \operatorname{Ly} \alpha$ & $\cdots$ \\
\hline $1253.051 \pm 0.023$ & $41 \pm 6$ & $25 \pm 5$ & $115 \pm 65$ & 45.3 & $\mathrm{i}: \operatorname{Ly} \alpha$ & $\ldots$ \\
\hline $1253.284 \pm 0.031$ & $97 \pm 7$ & $51 \pm 12$ & $375 \pm 117$ & 146.8 & $\mathrm{i}: \operatorname{Ly} \alpha$ & h:SII1253.8 \\
\hline
\end{tabular}


Table 9 -Continued

\begin{tabular}{|c|c|c|c|c|c|c|}
\hline $\begin{array}{l}\text { Wavelength } \\
(\AA)\end{array}$ & $\begin{array}{l}\text { Velocity } \\
\left(\mathrm{km} \mathrm{s}^{-1}\right)\end{array}$ & $\begin{array}{c}b \\
\left(\mathrm{~km} \mathrm{~s}^{-1}\right)\end{array}$ & $\begin{array}{c}\mathcal{W} \\
(\mathrm{m} \AA)\end{array}$ & $\begin{array}{l}\mathrm{SL} \\
(\sigma)\end{array}$ & $\mathrm{Id}$ & Alt Id \\
\hline $1253.669 \pm 0.045$ & $-34 \pm 11$ & $32 \pm 16$ & $107 \pm 83$ & 39.8 & g:SII1253.8 & $\ldots$ \\
\hline $1253.861 \pm 0.040$ & $235 \pm 10$ & $26 \pm 6$ & $94 \pm 53$ & 35.4 & $\mathrm{i}: \operatorname{Ly} \alpha$ & $\cdots$ \\
\hline \multicolumn{7}{|c|}{ Markarian 290} \\
\hline $1232.797 \pm 0.064$ & $4224 \pm 16$ & $21 \pm 29$ & $41 \pm 49$ & 3.6 & $\operatorname{Ly} \alpha$ & $\ldots$ \\
\hline $1234.597 \pm 0.027$ & $4667 \pm 7$ & $26 \pm 8$ & $60 \pm 18$ & 7.2 & $\operatorname{Ly} \alpha$ & i:NI1199.5? \\
\hline $1235.764 \pm 0.044$ & $4955 \pm 11$ & $26 \pm 18$ & $28 \pm 19$ & 3.4 & $\operatorname{Ly} \alpha$ & $\ldots$ \\
\hline $1238.346 \pm 0.035$ & $-110 \pm 8$ & $18 \pm 14$ & $23 \pm 13$ & 3.0 & h:NV1238.8 & $\mathrm{g}: \mathrm{NV} 1238.8 ?$ \\
\hline $1240.335 \pm 0.035$ & $-14 \pm 8$ & $22 \pm 12$ & $26 \pm 13$ & 3.6 & g:MgII1240.4 & $\cdots$ \\
\hline $1242.289 \pm 0.028$ & $25 \pm 7$ & $10 \pm 13$ & $22 \pm 10$ & 3.5 & i:SiIII1206.5 & h:NV1242.8 \\
\hline $1244.408 \pm 0.032$ & $7087 \pm 8$ & $23 \pm 11$ & $23 \pm 10$ & 4.3 & $\operatorname{Ly} \alpha$ & $\cdots$ \\
\hline $1245.536 \pm 0.025$ & $7365 \pm 6$ & $11 \pm 9$ & $21 \pm 7$ & 4.2 & $\operatorname{Ly} \alpha$ & $\cdots$ \\
\hline $1245.869 \pm 0.026$ & $7447 \pm 6$ & $8 \pm 12$ & $18 \pm 7$ & 3.6 & $\operatorname{Ly} \alpha$ & $\cdots$ \\
\hline $1248.079 \pm 0.028$ & $-850 \pm 7$ & $20 \pm 9$ & $22 \pm 9$ & 4.9 & $\mathrm{i}: \operatorname{Ly} \alpha$ & $\cdots$ \\
\hline $1249.349 \pm 0.029$ & $-545 \pm 7$ & $50 \pm 26$ & $25 \pm 23$ & 6.4 & $\mathrm{i}: \operatorname{Ly} \alpha$ & $\cdots$ \\
\hline $1249.579 \pm 0.029$ & $-490 \pm 7$ & $97 \pm 12$ & $225 \pm 48$ & 60.4 & $\mathrm{i}: \operatorname{Ly} \alpha$ & $\ldots$ \\
\hline $1250.019 \pm 0.029$ & $-139 \pm 7$ & $31 \pm 22$ & $19 \pm 23$ & 5.6 & h:SII1250.6 & $\mathrm{i}: \operatorname{Ly} \alpha$ \\
\hline $1250.549 \pm 0.031$ & $-8 \pm 7$ & $94 \pm 6$ & $558 \pm 65$ & 186.2 & g:SII1250.6 & $\cdots$ \\
\hline $1251.115 \pm 0.039$ & $-122 \pm 9$ & $99 \pm 3$ & $475 \pm 37$ & 185.6 & $\mathrm{i}: \operatorname{Ly} \alpha$ & $\ldots$ \\
\hline $1251.664 \pm 0.025$ & $9 \pm 6$ & $50 \pm 4$ & $105 \pm 17$ & 45.2 & $\mathrm{i}: \operatorname{Ly} \alpha$ & $\cdots$ \\
\hline $1252.109 \pm 0.029$ & $116 \pm 7$ & $61 \pm 12$ & $71 \pm 17$ & 31.3 & $\mathrm{i}: \operatorname{Ly} \alpha$ & $\ldots$ \\
\hline $1252.479 \pm 0.029$ & $204 \pm 7$ & $35 \pm 6$ & $33 \pm 10$ & 14.5 & $\mathrm{i}: \operatorname{Ly} \alpha$ & $\cdots$ \\
\hline $1253.327 \pm 0.029$ & $-113 \pm 7$ & $28 \pm 5$ & $34 \pm 6$ & 13.7 & h:SII1253.8 & $\ldots$ \\
\hline $1253.804 \pm 0.023$ & $-2 \pm 5$ & $36 \pm 3$ & $181 \pm 10$ & 67.8 & g:SII1253.8 & $\cdots$ \\
\hline $1254.353 \pm 0.029$ & $653 \pm 7$ & $81 \pm 29$ & $50 \pm 24$ & 17.3 & $\mathrm{i}: \operatorname{Ly} \alpha$ & $\ldots$ \\
\hline $1254.653 \pm 0.029$ & $725 \pm 7$ & $35 \pm 14$ & $24 \pm 12$ & 7.8 & $\mathrm{i}: \operatorname{Ly} \alpha$ & $\ldots$ \\
\hline $1254.953 \pm 0.029$ & $797 \pm 7$ & $23 \pm 13$ & $16 \pm 10$ & 4.8 & $\mathrm{i}: \operatorname{Ly} \alpha$ & $\cdots$ \\
\hline $1259.058 \pm 0.029$ & $-110 \pm 7$ & $39 \pm 7$ & $72 \pm 16$ & 15.2 & h:SII1259.5 & $\ldots$ \\
\hline $1259.328 \pm 0.029$ & $-46 \pm 7$ & $17 \pm 6$ & $40 \pm 13$ & 8.4 & g:SII1259.5 & $\mathrm{i}: \operatorname{Ly} \alpha$ \\
\hline $1259.535 \pm 0.023$ & $4 \pm 5$ & $24 \pm 3$ & $153 \pm 14$ & 31.7 & g:SII1259.5 & $\ldots$ \\
\hline $1260.008 \pm 0.029$ & $-117 \pm 7$ & $63 \pm 3$ & $479 \pm 28$ & 97.3 & h:SiII+FeII1260.5 & $\mathrm{g}: \mathrm{SiII}+\mathrm{Fe} I I 1260.5 ?$ \\
\hline
\end{tabular}


Table 9 -Continued

\begin{tabular}{|c|c|c|c|c|c|c|}
\hline $\begin{array}{c}\text { Wavelength } \\
(\AA)\end{array}$ & $\begin{array}{l}\text { Velocity } \\
\left(\mathrm{km} \mathrm{s}^{-1}\right)\end{array}$ & $\begin{array}{c}b \\
\left(\mathrm{~km} \mathrm{~s}^{-1}\right)\end{array}$ & $\begin{array}{c}\mathcal{W} \\
(\mathrm{m} \AA)\end{array}$ & $\begin{array}{l}\mathrm{SL} \\
(\sigma)\end{array}$ & Id & Alt Id \\
\hline $1260.464 \pm 0.027$ & $-9 \pm 6$ & $55 \pm 4$ & $399 \pm 45$ & 79.5 & $\mathrm{~g}: \mathrm{SiII}+\mathrm{FeII} 1260.5$ & $\cdots$ \\
\hline \multicolumn{7}{|c|}{ Markarian 335} \\
\hline $1223.637 \pm 0.026$ & $1965 \pm 6$ & $75 \pm 7$ & $229 \pm 30$ & 28.1 & $\operatorname{Ly} \alpha$ & $\ldots$ \\
\hline $1224.974 \pm 0.049$ & $2295 \pm 12$ & $73 \pm 17$ & $81 \pm 26$ & 10.2 & $\operatorname{Ly} \alpha$ & $\ldots$ \\
\hline $1232.979 \pm 0.057$ & $4268 \pm 14$ & $51 \pm 20$ & $33 \pm 16$ & 5.1 & $\operatorname{Ly} \alpha$ & $\ldots$ \\
\hline $1241.093 \pm 0.026$ & $6269 \pm 6$ & $75 \pm 6$ & $130 \pm 14$ & 30.3 & $\operatorname{Ly} \alpha$ & $\ldots$ \\
\hline $1250.584 \pm 0.023$ & $0 \pm 6$ & $58 \pm 4$ & $126 \pm 13$ & 35.6 & g:SII1250.6 & $\ldots$ \\
\hline $1253.786 \pm 0.023$ & $-6 \pm 6$ & $67 \pm 4$ & $197 \pm 15$ & 41.3 & g:SII1253.8 & $\cdots$ \\
\hline \multicolumn{7}{|c|}{ Markarian 421} \\
\hline $1227.977 \pm 0.025$ & $3035 \pm 6$ & $35 \pm 5$ & $86 \pm 15$ & 12.4 & $\operatorname{Ly} \alpha$ & $\ldots$ \\
\hline $1238.626 \pm 0.034$ & $-47 \pm 8$ & $19 \pm 13$ & $20 \pm 11$ & 3.2 & $\mathrm{~g}: \mathrm{NV} 1238.8$ & h:NV1238.8 \\
\hline $1250.385 \pm 0.031$ & $-52 \pm 7$ & $11 \pm 16$ & $28 \pm 31$ & 4.5 & h:SII1250.6 & $\ldots$ \\
\hline $1250.564 \pm 0.052$ & $-5 \pm 12$ & $35 \pm 14$ & $83 \pm 40$ & 13.2 & $\mathrm{~g}: \mathrm{SII} 1250.6$ & $\ldots$ \\
\hline $1253.578 \pm 0.031$ & $-53 \pm 8$ & $11 \pm 13$ & $33 \pm 29$ & 4.8 & h:SII1253.8 & $\ldots$ \\
\hline $1253.764 \pm 0.031$ & $-11 \pm 7$ & $33 \pm 7$ & $155 \pm 38$ & 22.7 & g:SII1253.8 & $\ldots$ \\
\hline $1256.928 \pm 0.051$ & $1140 \pm 12$ & $17 \pm 15$ & $23 \pm 27$ & 3.7 & $\mathrm{i}: \operatorname{Ly} \alpha$ & $\ldots$ \\
\hline $1257.127 \pm 0.029$ & $1188 \pm 7$ & $30 \pm 35$ & $22 \pm 27$ & 3.7 & $\mathrm{i}: \operatorname{Ly} \alpha$ & $\ldots$ \\
\hline \multicolumn{7}{|c|}{ Markarian 501} \\
\hline $1234.572 \pm 0.039$ & $4661 \pm 10$ & $60 \pm 13$ & $161 \pm 43$ & 11.1 & $\operatorname{Ly} \alpha$ & Absorber ' $A$ ' \\
\hline $1238.757 \pm 0.062$ & $-16 \pm 15$ & $31 \pm 25$ & $47 \pm 41$ & 3.4 & $\mathrm{~g}: \mathrm{NV} 1238.8$ & $\operatorname{Ly} \alpha$ \\
\hline $1239.968 \pm 0.029$ & $5992 \pm 7$ & $59 \pm 39$ & $55 \pm 46$ & 4.2 & $\operatorname{Ly} \alpha$ & Absorber ' $\mathbf{B}$ ' \\
\hline $1242.610 \pm 0.243$ & $-47 \pm 59$ & $74 \pm 70$ & $46 \pm 55$ & 3.1 & $\mathrm{~g}: \mathrm{NV} 1242.8$ & $\operatorname{Ly} \alpha$ \\
\hline $1246.177 \pm 0.069$ & $7523 \pm 17$ & $48 \pm 26$ & $53 \pm 36$ & 4.0 & $\operatorname{Ly} \alpha$ & Absorber ' $\mathrm{C}$ ' \\
\hline $1250.592 \pm 0.063$ & $2 \pm 15$ & $42 \pm 22$ & $99 \pm 64$ & 7.1 & $\mathrm{~g}: \mathrm{SII} 1250.6$ & $\ldots$ \\
\hline $1251.152 \pm 0.029$ & $8750 \pm 7$ & $77 \pm 49$ & $66 \pm 57$ & 4.9 & $\operatorname{Ly} \alpha$ & h:SII1250.6 \\
\hline $1253.069 \pm 0.264$ & $-175 \pm 63$ & $99 \pm 3$ & $64 \pm 33$ & 4.7 & h:SII1253.8 & $\mathrm{i}: \operatorname{Ly} \alpha$ \\
\hline
\end{tabular}


Table 9-Continued

\begin{tabular}{|c|c|c|c|c|c|c|}
\hline $\begin{array}{c}\text { Wavelength } \\
(\AA)\end{array}$ & $\begin{array}{l}\text { Velocity } \\
\left(\mathrm{km} \mathrm{s}^{-1}\right)\end{array}$ & $\begin{array}{c}b \\
\left(\mathrm{~km} \mathrm{~s}^{-1}\right)\end{array}$ & $\begin{array}{c}\mathcal{W} \\
(\mathrm{m} \AA)\end{array}$ & $\begin{array}{l}\mathrm{SL} \\
(\sigma)\end{array}$ & $\mathrm{Id}$ & Alt Id \\
\hline $1253.803 \pm 0.051$ & $-2 \pm 12$ & $85 \pm 16$ & $235 \pm 61$ & 17.5 & g:SII1253.8 & \\
\hline $1254.773 \pm 0.029$ & $-434 \pm 7$ & $99 \pm 3$ & $70 \pm 28$ & 5.4 & $\mathrm{i}: \operatorname{Ly} \alpha$ & h:SII1253.8 \\
\hline \multicolumn{7}{|c|}{ Markarian 509} \\
\hline $1226.050 \pm 0.025$ & $2560 \pm 6$ & $40 \pm 5$ & $209 \pm 32$ & 14.4 & $\operatorname{Ly} \alpha$ & $\cdots$ \\
\hline $1238.669 \pm 0.029$ & $-32 \pm 7$ & $16 \pm 29$ & $14 \pm 17$ & 3.0 & h:NV1238.8 & $\mathrm{g}: \mathrm{NV} 1238.8$ \\
\hline $1238.924 \pm 0.029$ & $25 \pm 7$ & $56 \pm 48$ & $100 \pm 111$ & 21.1 & $\mathrm{~g}: \mathrm{NV} 1238.8$ & h:MgII1239.9 \\
\hline $1239.182 \pm 0.029$ & $-180 \pm 7$ & $21 \pm 18$ & $24 \pm 29$ & 5.1 & h:MgII1239.9 & $\ldots$ \\
\hline $1239.951 \pm 0.029$ & $6 \pm 7$ & $18 \pm 9$ & $24 \pm 9$ & 5.1 & g:MgII1239.9 & h:MgII1240.4 \\
\hline $1242.874 \pm 1.206$ & $17 \pm 291$ & $45 \pm 46$ & $29 \pm 35$ & 7.2 & g:NV1242.8 & h:NV1242.8 \\
\hline $1243.060 \pm 0.734$ & $62 \pm 177$ & $35 \pm 38$ & $17 \pm 21$ & 4.3 & h:NV1242.8 & $\mathrm{g}: \mathrm{NV} 1242.8 ?$ \\
\hline $1250.314 \pm 0.029$ & $-69 \pm 7$ & $24 \pm 9$ & $20 \pm 7$ & 7.2 & h:SII1250.6 & $\cdots$ \\
\hline $1250.583 \pm 0.023$ & $0 \pm 5$ & $23 \pm 3$ & $86 \pm 7$ & 31.1 & g:SII1250.6 & $\ldots$ \\
\hline $1250.819 \pm 0.029$ & $56 \pm 7$ & $42 \pm 5$ & $58 \pm 8$ & 21.3 & h:SII1250.6 & $\mathrm{g}: \mathrm{SII} 1250.6 ?$ \\
\hline $1253.561 \pm 0.029$ & $-57 \pm 7$ & $41 \pm 9$ & $27 \pm 7$ & 11.7 & h:SII1253.8 & $\mathrm{g}: \mathrm{SII} 1253.8 ?$ \\
\hline $1253.832 \pm 0.023$ & $5 \pm 5$ & $28 \pm 3$ & $115 \pm 17$ & 50.5 & g:SII1253.8 & h:SII1253.8? \\
\hline $1254.079 \pm 0.032$ & $64 \pm 8$ & $40 \pm 6$ & $85 \pm 19$ & 37.5 & h:SII1253.8 & $\mathrm{g}: \mathrm{SII} 1253.8 ?$ \\
\hline $1255.795 \pm 0.029$ & $-403 \pm 7$ & $46 \pm 5$ & $132 \pm 67$ & 64.4 & $\mathrm{i}: \operatorname{Ly} \alpha$ & $\cdots$ \\
\hline $1256.065 \pm 0.029$ & $-339 \pm 7$ & $44 \pm 14$ & $76 \pm 92$ & 37.9 & $\mathrm{i}: \operatorname{Ly} \alpha$ & $\cdots$ \\
\hline $1256.285 \pm 0.029$ & $-286 \pm 7$ & $93 \pm 31$ & $505 \pm 425$ & 147.7 & $\mathrm{i}: \operatorname{Ly} \alpha$ & $\cdots$ \\
\hline $1256.535 \pm 0.029$ & $-227 \pm 7$ & $63 \pm 60$ & $47 \pm 56$ & 3.3 & $\mathrm{i}: \operatorname{Ly} \alpha$ & $\ldots$ \\
\hline $1257.165 \pm 0.029$ & $-76 \pm 7$ & $69 \pm 21$ & $191 \pm 229$ & 61.9 & $\mathrm{i}: \operatorname{Ly} \alpha$ & $\cdots$ \\
\hline $1257.365 \pm 0.029$ & $-29 \pm 7$ & $65 \pm 62$ & $265 \pm 318$ & 121.5 & $\mathrm{i}: \operatorname{Ly} \alpha$ & $\ldots$ \\
\hline $1257.605 \pm 0.029$ & $29 \pm 7$ & $44 \pm 16$ & $140 \pm 168$ & 73.7 & $\mathrm{i}: \operatorname{Ly} \alpha$ & $\cdots$ \\
\hline $1257.785 \pm 0.029$ & $71 \pm 7$ & $28 \pm 17$ & $26 \pm 32$ & 13.9 & $\mathrm{i}: \operatorname{Ly} \alpha$ & $\ldots$ \\
\hline $1257.985 \pm 0.029$ & $119 \pm 7$ & $72 \pm 25$ & $449 \pm 240$ & 238.6 & $\mathrm{i}: \operatorname{Ly} \alpha$ & $\cdots$ \\
\hline $1258.365 \pm 0.029$ & $-270 \pm 7$ & $53 \pm 13$ & $237 \pm 151$ & 122.7 & h:SiII1259.5 & $\mathrm{i}: \operatorname{Ly} \alpha$ \\
\hline $1258.565 \pm 0.029$ & $-222 \pm 7$ & $33 \pm 8$ & $43 \pm 46$ & 22.6 & h:SiII1259.5 & $\mathrm{i}: \operatorname{Ly} \alpha$ \\
\hline $1259.550 \pm 0.026$ & $7 \pm 6$ & $60 \pm 4$ & $201 \pm 21$ & 107.8 & g:SII1259.5 & $\ldots$ \\
\hline $1259.858 \pm 0.029$ & $85 \pm 7$ & $34 \pm 19$ & $30 \pm 37$ & 16.1 & h:SII1259.5 & h:CI1260.7 \\
\hline $1260.257 \pm 0.029$ & $-48 \pm 7$ & $68 \pm 64$ & $43 \pm 52$ & 22.2 & h:SiII+FeII1260.5 & $\mathrm{g}: \mathrm{CI} 1260.7 ?$ \\
\hline
\end{tabular}


Table 9-Continued

\begin{tabular}{|c|c|c|c|c|c|c|}
\hline $\begin{array}{l}\text { Wavelength } \\
(\AA)\end{array}$ & $\begin{array}{l}\text { Velocity } \\
\left(\mathrm{km} \mathrm{s}^{-1}\right)\end{array}$ & $\begin{array}{c}b \\
\left(\mathrm{~km} \mathrm{~s}^{-1}\right)\end{array}$ & $\begin{array}{c}\mathcal{W} \\
(\mathrm{m} \AA)\end{array}$ & $\begin{array}{l}\mathrm{SL} \\
(\sigma)\end{array}$ & Id & Alt Id \\
\hline $1260.347 \pm 6.901$ & $-36 \pm 37$ & $71 \pm 68$ & $332 \pm 398$ & 170.8 & g:SiII+FeII1260.5 & $\cdots$ \\
\hline $1260.710 \pm 0.029$ & $50 \pm 7$ & $72 \pm 4$ & $372 \pm 73$ & 186.9 & h:SiII+FeII1260.5 & g:CI1260.7 \\
\hline \multicolumn{7}{|c|}{ Markarian 817} \\
\hline $1223.507 \pm 0.037$ & $1933 \pm 9$ & $34 \pm 13$ & $29 \pm 13$ & 5.3 & $\operatorname{Ly} \alpha$ & $\cdots$ \\
\hline $1224.172 \pm 0.023$ & $2097 \pm 5$ & $40 \pm 4$ & $135 \pm 15$ & 25.3 & $\operatorname{Ly} \alpha$ & $\cdots$ \\
\hline $1234.657 \pm 0.041$ & $4682 \pm 10$ & $40 \pm 15$ & $23 \pm 11$ & 5.6 & $\operatorname{Ly} \alpha$ & $\ldots$ \\
\hline $1236.303 \pm 0.023$ & $5088 \pm 6$ & $84 \pm 4$ & $207 \pm 14$ & 56.3 & $\operatorname{Ly} \alpha$ & $\ldots$ \\
\hline $1236.902 \pm 0.027$ & $5236 \pm 7$ & $24 \pm 7$ & $25 \pm 7$ & 6.6 & $\operatorname{Ly} \alpha$ & $\ldots$ \\
\hline $1238.529 \pm 0.029$ & $-65 \pm 7$ & $75 \pm 28$ & $54 \pm 41$ & 13.5 & h:NV1238.8 & g:NV1238.8? \\
\hline $1238.809 \pm 0.029$ & $2 \pm 5$ & $40 \pm 26$ & $21 \pm 23$ & 5.2 & g:NV1238.8 & h:NV1238.8 \\
\hline $1239.159 \pm 0.029$ & $5793 \pm 7$ & $39 \pm 12$ & $34 \pm 13$ & 8.7 & $\operatorname{Ly} \alpha$ & $\cdots$ \\
\hline $1239.509 \pm 0.029$ & $-101 \pm 7$ & $46 \pm 18$ & $25 \pm 12$ & 6.3 & h:MgII1239.9 & g:MgII1239.9? \\
\hline $1241.034 \pm 0.024$ & $6255 \pm 6$ & $29 \pm 5$ & $37 \pm 8$ & 11.6 & $\operatorname{Ly} \alpha$ & $\ldots$ \\
\hline $1242.740 \pm 0.029$ & $-15 \pm 7$ & $25 \pm 32$ & $10 \pm 12$ & 3.2 & g:NV1242.8 & h:NV1242.8 \\
\hline $1245.395 \pm 0.051$ & $7330 \pm 13$ & $51 \pm 18$ & $17 \pm 7$ & 5.9 & $\operatorname{Ly} \alpha$ & $\cdots$ \\
\hline $1247.294 \pm 0.044$ & $7799 \pm 11$ & $56 \pm 16$ & $28 \pm 9$ & 10.1 & $\operatorname{Ly} \alpha$ & $\cdots$ \\
\hline $1249.477 \pm 0.041$ & $-1023 \pm 10$ & $26 \pm 9$ & $36 \pm 17$ & 13.8 & $\mathrm{i}: \operatorname{Ly} \alpha$ & $\cdots$ \\
\hline $1249.694 \pm 0.077$ & $-971 \pm 18$ & $28 \pm 21$ & $20 \pm 19$ & 7.7 & $\mathrm{i}: \operatorname{Ly} \alpha$ & $\cdots$ \\
\hline $1250.071 \pm 0.034$ & $-127 \pm 8$ & $24 \pm 13$ & $13 \pm 6$ & 5.1 & h:SII1250.6 & $\ldots$ \\
\hline $1250.415 \pm 0.029$ & $-44 \pm 7$ & $30 \pm 11$ & $26 \pm 9$ & 9.8 & h:SII1250.6 & $\cdots$ \\
\hline $1250.568 \pm 0.023$ & $-4 \pm 5$ & $16 \pm 4$ & $47 \pm 6$ & 18.0 & g:SII1250.6 & $\ldots$ \\
\hline $1251.752 \pm 0.025$ & $-479 \pm 6$ & $18 \pm 7$ & $14 \pm 4$ & 6.0 & $\mathrm{i}: \operatorname{Ly} \alpha$ & $\cdots$ \\
\hline $1253.355 \pm 0.027$ & $-106 \pm 6$ & $27 \pm 12$ & $32 \pm 13$ & 12.9 & h:SII1253.8 & $\cdots$ \\
\hline $1253.653 \pm 0.029$ & $-35 \pm 7$ & $27 \pm 8$ & $53 \pm 16$ & 21.7 & h:SII1253.8 & $\cdots$ \\
\hline $1253.810 \pm 0.023$ & $0 \pm 5$ & $19 \pm 4$ & $76 \pm 8$ & 31.3 & g:SII1253.8 & $\cdots$ \\
\hline
\end{tabular}

PKS 2155-304: PRE-COSTAR

\begin{tabular}{lllllll}
\hline $1226.345 \pm 0.060$ & $2632 \pm 15$ & $61 \pm 33$ & $42 \pm 40$ & 9.2 & Ly $\alpha$ & $\ldots$ \\
$1226.964 \pm 0.065$ & $2785 \pm 16$ & $64 \pm 26$ & $36 \pm 22$ & 7.9 & Ly $\alpha$ & z:SiIII1206.5
\end{tabular}


Table 9-Continued

\begin{tabular}{|c|c|c|c|c|c|c|}
\hline $\begin{array}{l}\text { Wavelength } \\
\qquad(\AA)\end{array}$ & $\begin{array}{l}\text { Velocity } \\
\left(\mathrm{km} \mathrm{s}^{-1}\right)\end{array}$ & $\begin{array}{c}b \\
\left(\mathrm{~km} \mathrm{~s}^{-1}\right)\end{array}$ & $\begin{array}{c}\mathcal{W} \\
(\mathrm{m} \AA)\end{array}$ & $\begin{array}{l}\mathrm{SL} \\
(\sigma)\end{array}$ & $\mathrm{Id}$ & Alt Id \\
\hline $1232.016 \pm 0.049$ & $4031 \pm 12$ & $39 \pm 17$ & $21 \pm 11$ & 4.2 & $\operatorname{Ly} \alpha$ & $\cdots$ \\
\hline $1234.767 \pm 0.051$ & $4709 \pm 12$ & $32 \pm 24$ & $15 \pm 14$ & 3.3 & $\operatorname{Ly} \alpha$ & $\cdots$ \\
\hline $1235.748 \pm 0.029$ & $4951 \pm 7$ & $68 \pm 15$ & $64 \pm 23$ & 14.3 & $\operatorname{Ly} \alpha$ & $\cdots$ \\
\hline $1235.998 \pm 0.029$ & $5013 \pm 7$ & $58 \pm 11$ & $82 \pm 22$ & 18.1 & $\operatorname{Ly} \alpha$ & $\cdots$ \\
\hline $1236.426 \pm 0.029$ & $5119 \pm 7$ & $80 \pm 5$ & $218 \pm 20$ & 48.1 & $\operatorname{Ly} \alpha$ & $\cdots$ \\
\hline $1238.451 \pm 0.029$ & $5618 \pm 7$ & $33 \pm 14$ & $29 \pm 15$ & 6.7 & $\operatorname{Ly} \alpha$ & $\cdots$ \\
\hline $1238.673 \pm 0.031$ & $5673 \pm 8$ & $30 \pm 12$ & $39 \pm 16$ & 9.1 & $\operatorname{Ly} \alpha$ & $\ldots$ \\
\hline $1238.869 \pm 0.029$ & $17 \pm 7$ & $31 \pm 11$ & $31 \pm 13$ & 7.2 & g:NV1238.8 & $\ldots$ \\
\hline $1239.802 \pm 0.045$ & $-30 \pm 11$ & $52 \pm 16$ & $32 \pm 13$ & 7.3 & g:MgII1239.9 & $\cdots$ \\
\hline $1240.392 \pm 0.055$ & $-1 \pm 13$ & $41 \pm 21$ & $18 \pm 12$ & 4.2 & g:MgII1240.4 & $\operatorname{Ly} \alpha$ \\
\hline $1242.849 \pm 0.029$ & $11 \pm 7$ & $47 \pm 30$ & $20 \pm 16$ & 4.0 & g:NV1242.8 & $\operatorname{Ly} \alpha$ \\
\hline $1246.990 \pm 0.029$ & $7724 \pm 7$ & $31 \pm 35$ & $13 \pm 16$ & 3.0 & $\operatorname{Ly} \alpha$ & $\cdots$ \\
\hline $1247.510 \pm 0.029$ & $7852 \pm 7$ & $30 \pm 31$ & $13 \pm 16$ & 3.1 & $\operatorname{Ly} \alpha$ & $\cdots$ \\
\hline $1250.572 \pm 0.026$ & $-3 \pm 6$ & $51 \pm 6$ & $80 \pm 11$ & 18.4 & g:SII1250.6 & $\cdots$ \\
\hline $1253.790 \pm 0.023$ & $-5 \pm 6$ & $53 \pm 4$ & $126 \pm 13$ & 27.9 & g:SII1253.8 & $\cdots$ \\
\hline $1255.084 \pm 0.041$ & $9720 \pm 10$ & $24 \pm 16$ & $13 \pm 9$ & 3.0 & $\operatorname{Ly} \alpha$ & $\cdots$ \\
\hline $1256.636 \pm 0.042$ & $10102 \pm 10$ & $25 \pm 15$ & $14 \pm 8$ & 3.3 & $\operatorname{Ly} \alpha$ & $\cdots$ \\
\hline
\end{tabular}

PKS 2155-304: POST-COSTAR

\begin{tabular}{lcccccc}
\hline $1259.502 \pm 0.023$ & $-4 \pm 5$ & $21 \pm 4$ & $133 \pm 19$ & 14.9 & g:SII1259.5 & h:CI1260.7 \\
$1259.848 \pm 0.028$ & $-155 \pm 7$ & $31 \pm 9$ & $76 \pm 23$ & 8.5 & h:SiII+FeII1260.5 & g:SII1259.5? \\
$1260.351 \pm 0.028$ & $-35 \pm 7$ & $22 \pm 12$ & $68 \pm 59$ & 7.7 & g:SiII+FeII1260.5 & $\ldots$ \\
$1260.513 \pm 0.036$ & $3 \pm 6$ & $61 \pm 6$ & $412 \pm 77$ & 46.2 & g:SiII+FeII1260.5 & g:CI1260.7? \\
$1264.806 \pm 0.058$ & $12117 \pm 14$ & $39 \pm 20$ & $31 \pm 19$ & 3.6 & Ly $\alpha$ & $\ldots$ \\
$1270.784 \pm 0.027$ & $13591 \pm 6$ & $39 \pm 6$ & $101 \pm 18$ & 12.5 & Ly $\alpha$ & $\ldots$ \\
$1281.375 \pm 0.024$ & $16203 \pm 5$ & $58 \pm 3$ & $346 \pm 23$ & 44.8 & Ly $\alpha$ & $\ldots$ \\
$1281.867 \pm 0.061$ & $16325 \pm 15$ & $49 \pm 22$ & $62 \pm 34$ & 8.2 & Ly $\alpha$ & $\ldots$ \\
$1284.301 \pm 0.030$ & $16925 \pm 7$ & $19 \pm 13$ & $43 \pm 37$ & 5.7 & Ly $\alpha$ & $\ldots$ \\
$1284.497 \pm 0.039$ & $16973 \pm 9$ & $63 \pm 6$ & $389 \pm 68$ & 50.9 & Ly $\alpha$ & $\ldots$ \\
$1285.086 \pm 0.038$ & $17119 \pm 9$ & $87 \pm 11$ & $448 \pm 79$ & 57.9 & Ly $\alpha$ & $\ldots$ \\
$1287.497 \pm 0.024$ & $17713 \pm 6$ & $35 \pm 5$ & $139 \pm 21$ & 18.4 & Ly $\alpha$ & $\ldots$
\end{tabular}


Table 9 -Continued

\begin{tabular}{ccccccc}
\hline \hline $\begin{array}{c}\text { Wavelength } \\
(\AA)\end{array}$ & $\begin{array}{c}\text { Velocity } \\
\left(\mathrm{km} \mathrm{s}^{-1}\right)\end{array}$ & $\begin{array}{c}b \\
\left(\mathrm{~km} \mathrm{~s}^{-1}\right)\end{array}$ & $\begin{array}{c}\mathcal{W} \\
(\mathrm{m} \AA)\end{array}$ & $\begin{array}{r}\text { SL } \\
(\sigma)\end{array}$ & Id & Alt Id \\
\hline $1288.958 \pm 0.029$ & $18073 \pm 7$ & $47 \pm 8$ & $99 \pm 20$ & 13.2 & Ly $\alpha$ & $\ldots$ \\
\hline \multicolumn{7}{c}{ Q $1230+0115$} \\
\hline $1221.711 \pm 0.026$ & $1490 \pm 6$ & $21 \pm 8$ & $138 \pm 42$ & 6.3 & Ly $\alpha$ & $\ldots$ \\
$1222.425 \pm 0.035$ & $1666 \pm 9$ & $54 \pm 10$ & $385 \pm 94$ & 16.9 & Ly $\alpha$ & $\ldots$ \\
$1222.747 \pm 0.029$ & $1745 \pm 7$ & $40 \pm 12$ & $241 \pm 99$ & 10.9 & Ly $\alpha$ & $\ldots$ \\
$1223.211 \pm 0.051$ & $1860 \pm 13$ & $48 \pm 21$ & $142 \pm 81$ & 6.6 & Ly $\alpha$ & $\ldots$ \\
$1225.000 \pm 0.024$ & $2301 \pm 6$ & $55 \pm 6$ & $439 \pm 57$ & 23.9 & Ly $\alpha$ & $\ldots$ \\
$1236.045 \pm 0.041$ & $5025 \pm 10$ & $24 \pm 15$ & $53 \pm 31$ & 3.1 & Ly $\alpha$ & $\ldots$ \\
$1242.897 \pm 0.044$ & $6714 \pm 11$ & $19 \pm 19$ & $45 \pm 35$ & 3.2 & Ly $\alpha$ & g:NV1242.8 \\
$1246.254 \pm 0.049$ & $7542 \pm 12$ & $31 \pm 18$ & $44 \pm 27$ & 3.3 & Ly $\alpha$ & z:NV1238.8 \\
$1250.570 \pm 0.028$ & $-3 \pm 7$ & $27 \pm 7$ & $94 \pm 25$ & 7.0 & g:SII1250.6 & $\ldots$ \\
$1253.145 \pm 0.031$ & $9242 \pm 8$ & $72 \pm 8$ & $301 \pm 49$ & 24.8 & Ly $\alpha$ & $\ldots$ \\
$1253.783 \pm 0.029$ & $-7 \pm 7$ & $61 \pm 8$ & $327 \pm 69$ & 26.1 & g:SII1253.8 & $\ldots$ \\
\hline
\end{tabular}




\section{B. CfA Pie Diagrams and Nearest Neighbor Distances}

In this Appendix we present $\pm 7^{\circ}$ "pie diagrams" of our sightlines, and Table 10 which describes the three nearest known galaxies to each of our Ly $\alpha$ absorbers. In Figures 21-35, the coordinate along the slice of a pie diagram is the heliocentric recession velocity in $\mathrm{km} \mathrm{s}^{-1}$. The dashed line in each diagram marks the sightline. Sightlines terminating with dashed arrows indicate that the target lies beyond the extent of the pie diagram (3C 273, H 1821+643, and PKS 2155-304). Objects whose positions are included by the pie diagram are marked with a large circle indicating the AGN target. The smaller circles indicate the positions along the sight line of the Ly $\alpha$ absorbers; the middle-sized circles are the definite $(S L \geq 4 \sigma)$ detections, and the smallest circles are the possible $(3 \sigma \leq S L<4 \sigma)$ detections. Each symbol "c" is an individual galaxy in the merged CfA redshift catalog; the orientation of the "c" for each galaxy is conserved between the wedges. The "v" symbols indicate CfA catalog galaxies within $2^{\circ}$ of the sight line and are oriented consistently between the $\alpha$ and $\delta$ diagrams. Galaxies at $c z<500 \mathrm{~km} \mathrm{~s}^{-1}$ have been deleted from these plots.

Table 10 includes the following information by column: (1) the absorber type where $\mathrm{D}=$ Definite $(S L \geq 4 \sigma)$ or $\mathrm{P}=$ Possible $(3 \sigma \leq S L<4 \sigma)$; $(2)$ the heliocentric recession velocity of the absorber (non-relativistic $c z$ ) converted from LSR using the standard value for the local standard of rest (see $\S 5$ ); (3) the nearest galaxy name as given in the CfA catalog (Huchra et al. 1992); (4) the absorber-galaxy distance perpendicular to the line-of-sight (LOS) in $h_{70}^{-1} \mathrm{Mpc}$; (5) the nonrelativistic heliocentric recession velocity $\left(c z\right.$, in $\left.\mathrm{km} \mathrm{s}^{-1}\right)$ of the nearest galaxy as given in the CfA catalog; (6) the absorber-galaxy distance (in $h_{70}^{-1} \mathrm{Mpc}$ ) along the LOS, assuming a retarded Hubble flow $\left( \pm 300 \mathrm{~km} \mathrm{~s}^{-1}\right)$; (7) the Euclidean 3D absorber-galaxy distance (in $h_{70}^{-1} \mathrm{Mpc}$ ), assuming a retarded Hubble flow $\left( \pm 300 \mathrm{~km} \mathrm{~s}^{-1}\right.$; see $\S 5$ and Stocke et al. (1995)); and (8) the galaxy blue magnitude as given in the CfA Catalog.

As discussed in Paper III, the completeness of the CfA catalog varies with position on the sky. Therefore, direct conclusions about galaxy-absorber connections and "void" absorbers should not be derived from Table 10. However, if one adopts the very liberal definition of an "association" as any absorber within $500 h_{70}^{-1} \mathrm{kpc}$ of a CfA galaxy, and a "void" absorber as any galaxy whose nearest neighboring galaxy is $>6 h_{70}^{-1} \mathrm{Mpc}$ away, our $S L \geq 4 \sigma$ sample contains 20 galaxy-absorber "associations" and 21 galaxy "void" Ly $\alpha$ absorbers (using the total absorber-galaxy distances of column 7 in Table 10). Our $3 \sigma \leq S L<4 \sigma$ sample contains an additional two absorber-galaxy "associations" and four "void" absorbers. 


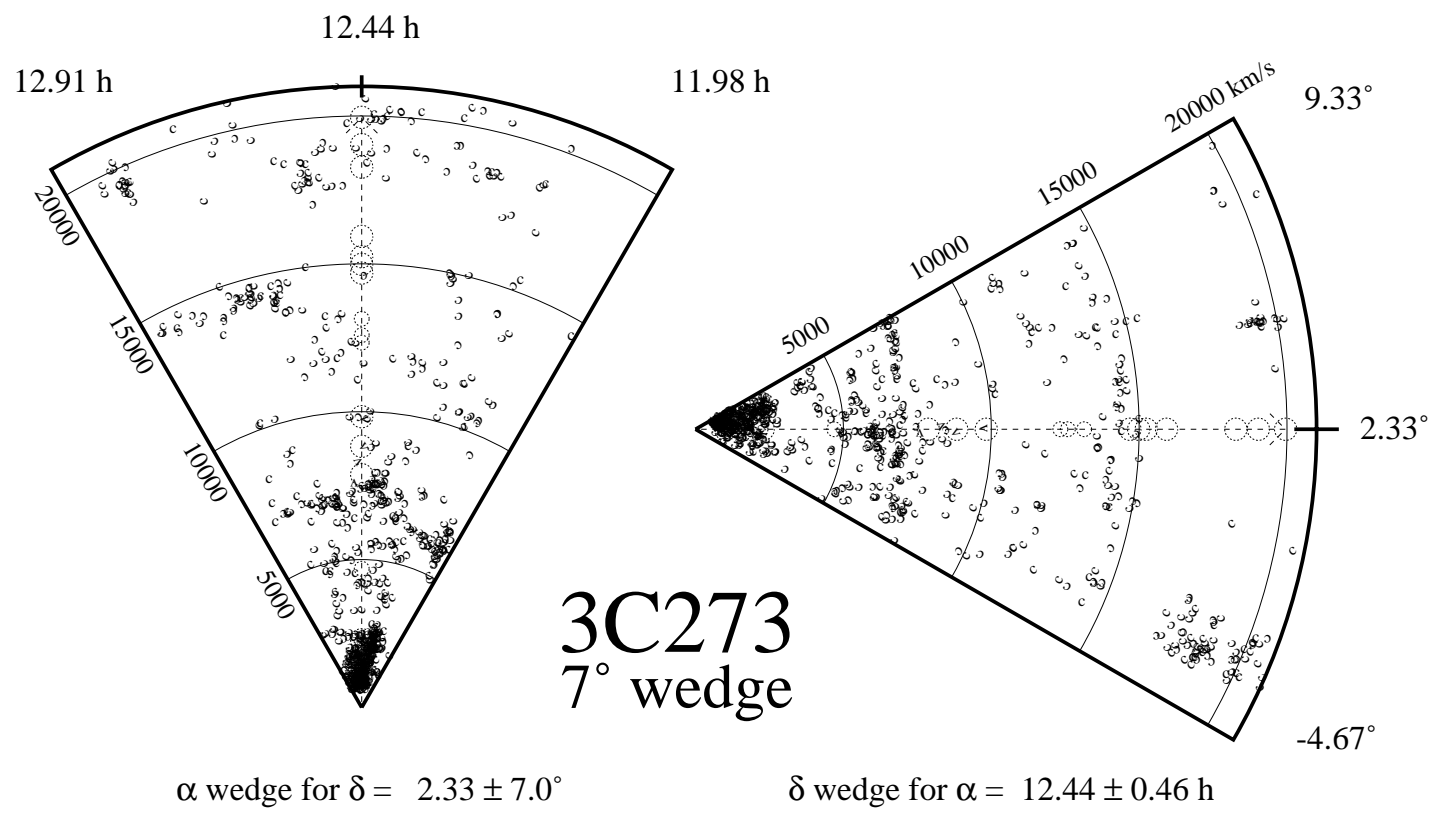

Fig. 21.- Right ascension $(\alpha)$ and declination $(\delta)$ pie diagram for the 3C 273 sightline.

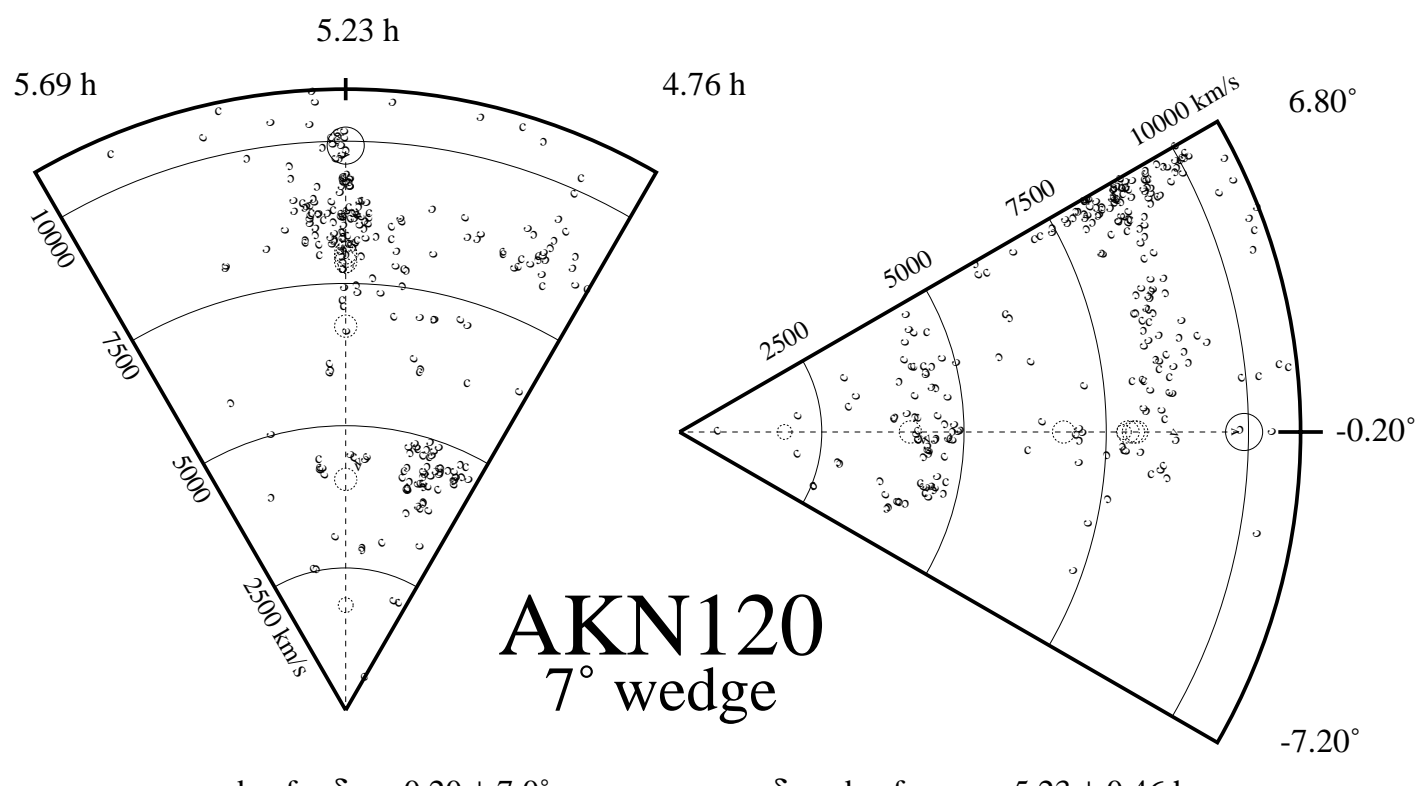

$\alpha$ wedge for $\delta=-0.20 \pm 7.0^{\circ}$

$\delta$ wedge for $\alpha=5.23 \pm 0.46 \mathrm{~h}$

Fig. 22.- Right ascension $(\alpha)$ and declination $(\delta)$ pie diagram for the Arakelian 120 sightline. 


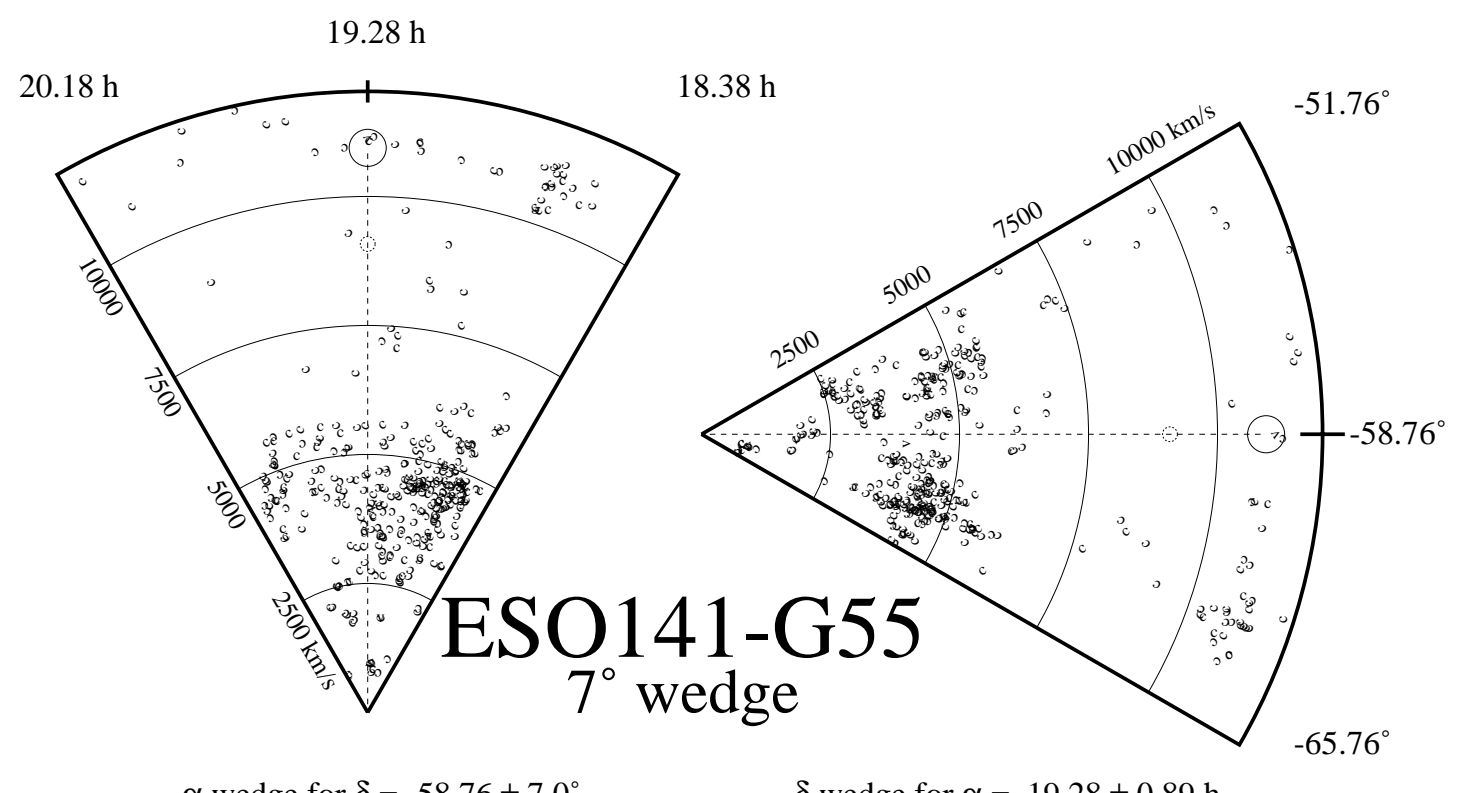

$\alpha$ wedge for $\delta=-58.76 \pm 7.0^{\circ}$

$\delta$ wedge for $\alpha=19.28 \pm 0.89 \mathrm{~h}$

Fig. 23. - Right ascension $(\alpha)$ and declination $(\delta)$ pie diagram for the ESO 141-G55 sightline.

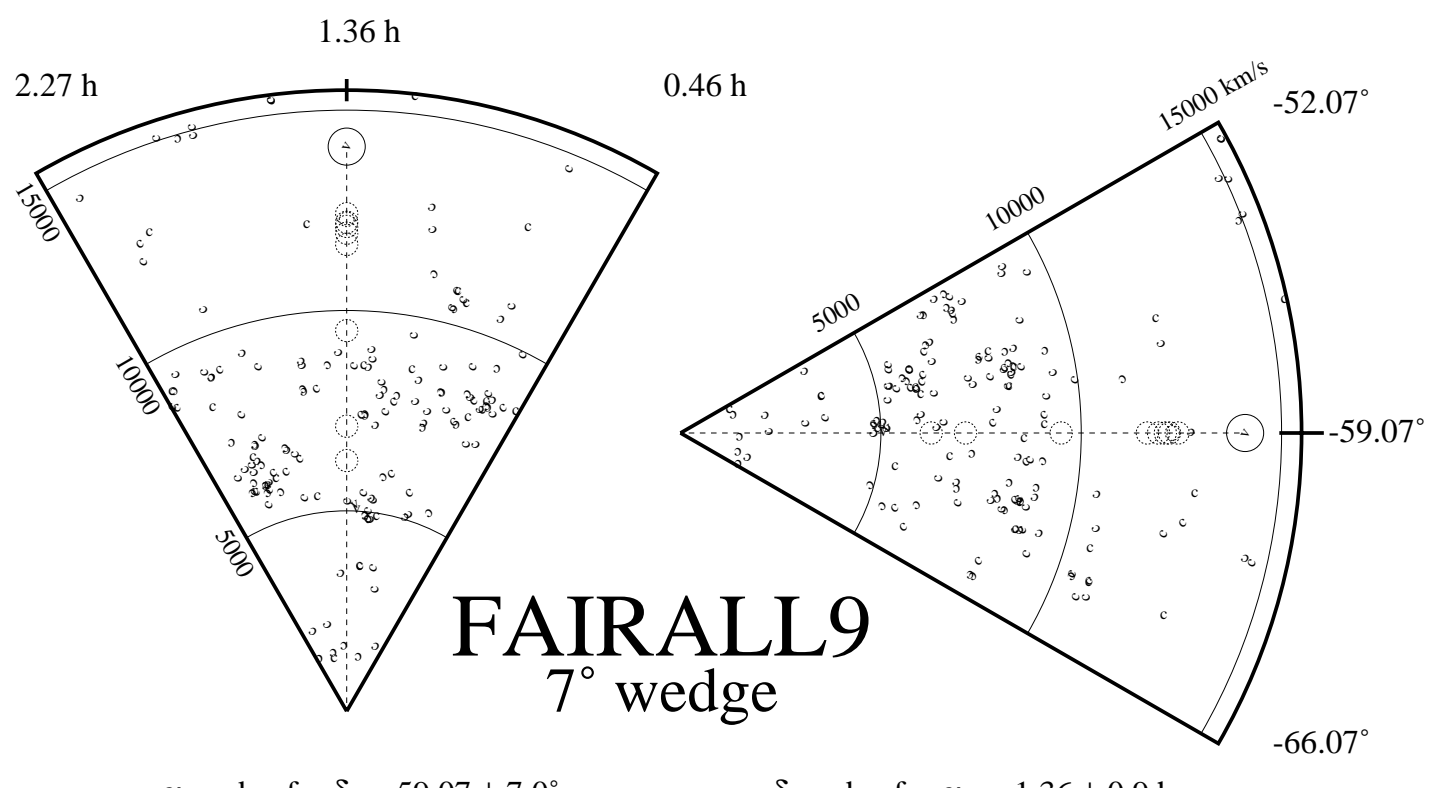

$\alpha$ wedge for $\delta=-59.07 \pm 7.0^{\circ}$

$\delta$ wedge for $\alpha=1.36 \pm 0.9 \mathrm{~h}$

Fig. 24.- Right ascension $(\alpha)$ and declination $(\delta)$ pie diagram for the FAIRALL 9 sightline. 


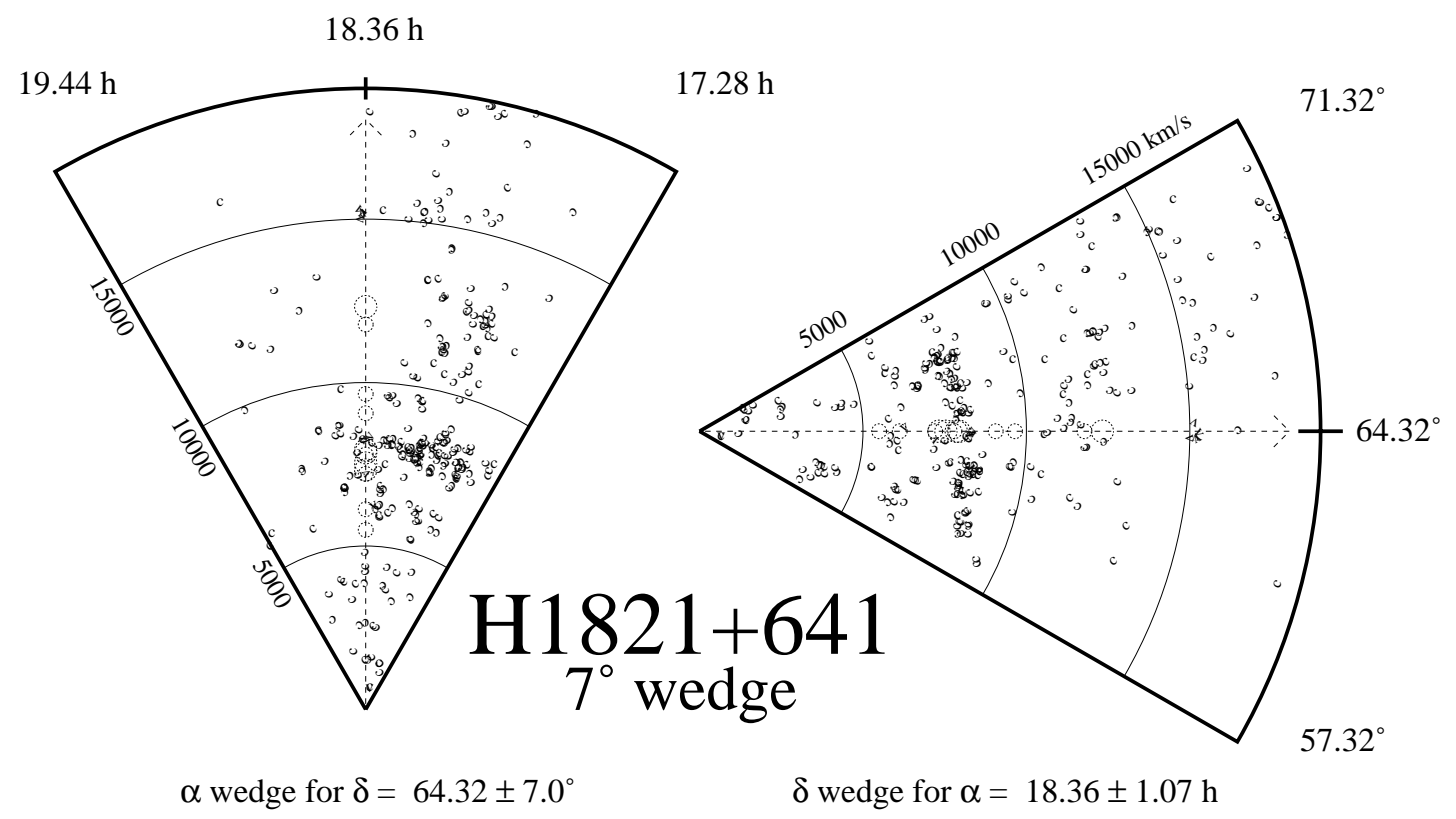

Fig. 25. - Right ascension $(\alpha)$ and declination $(\delta)$ pie diagram for the $\mathrm{H} 1821+643$ sightline.

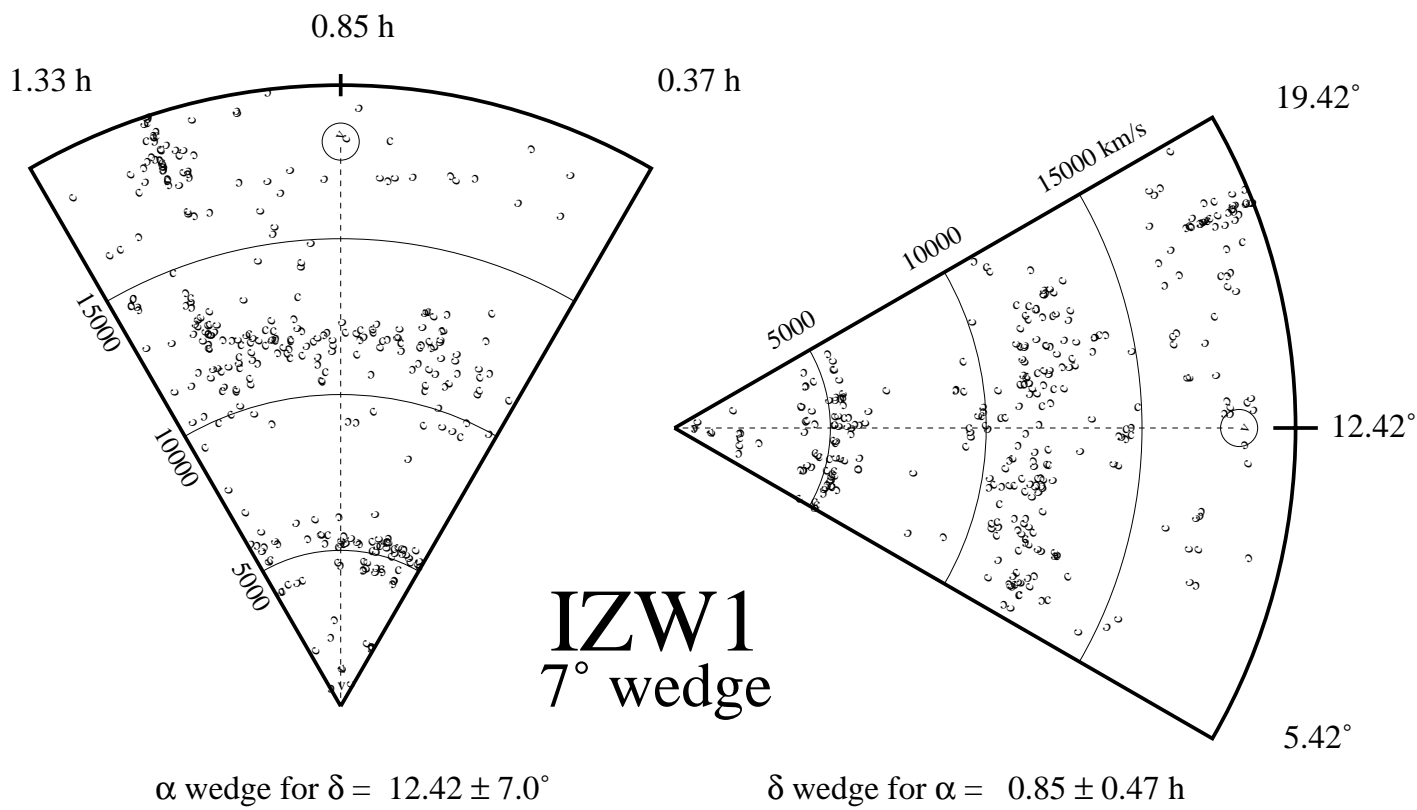

Fig. 26. - Right ascension $(\alpha)$ and declination $(\delta)$ pie diagram for the I ZW 1 sightline. 


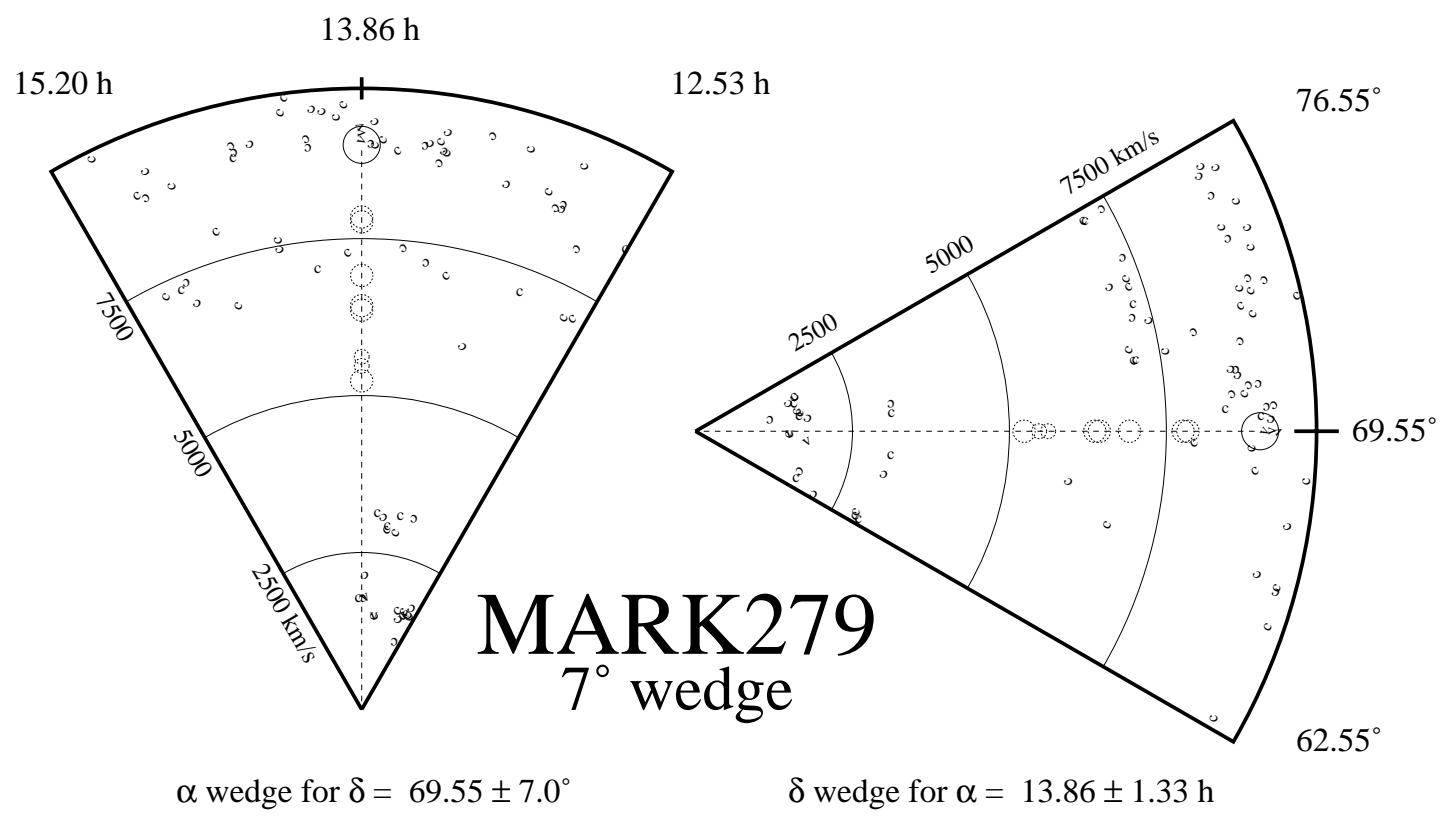

Fig. 27. - Right ascension $(\alpha)$ and declination $(\delta)$ pie diagram for the Markarian 279 sightline.

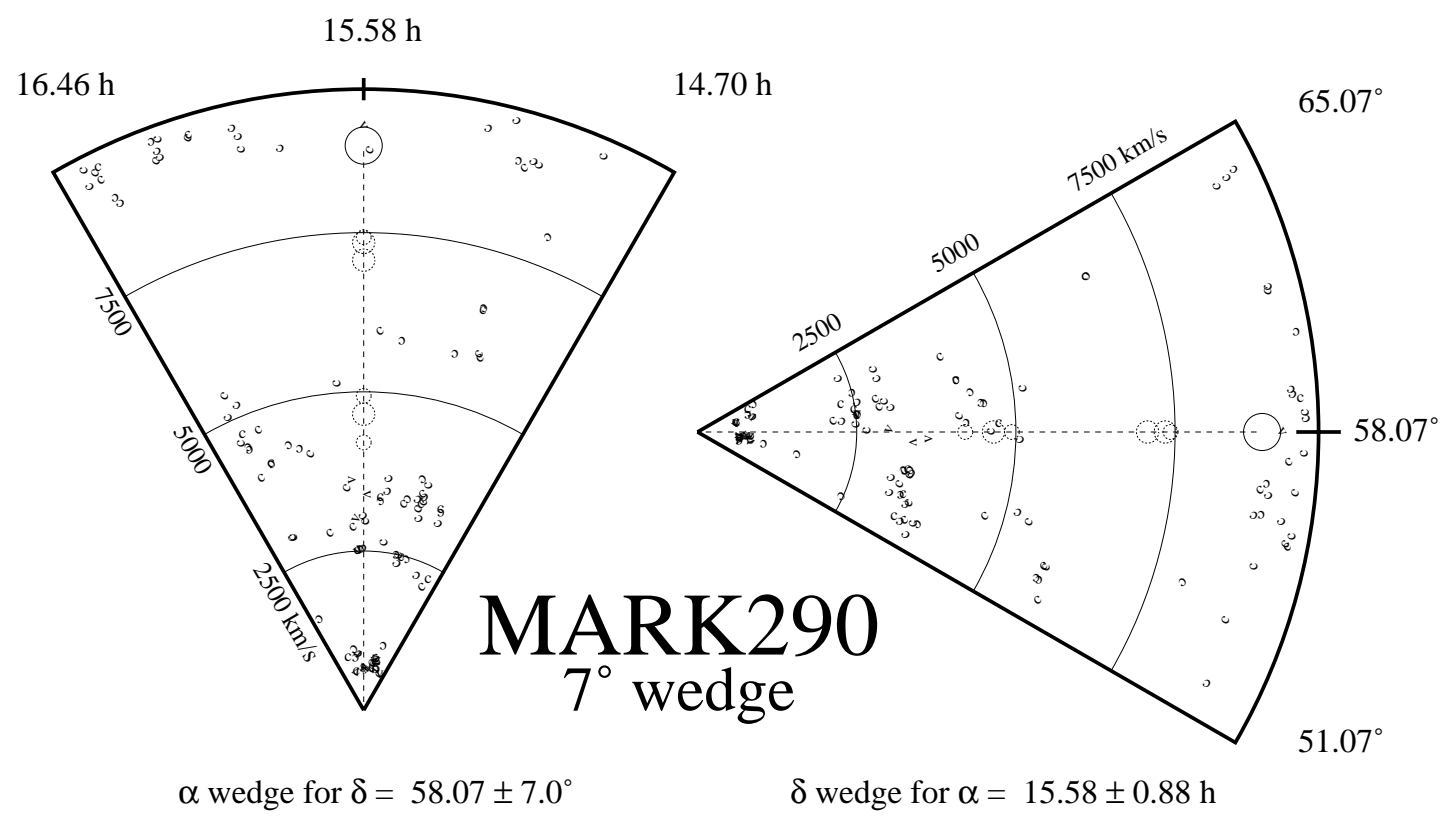

Fig. 28. - Right ascension $(\alpha)$ and declination $(\delta)$ pie diagram for the Markarian 290 sightline. 


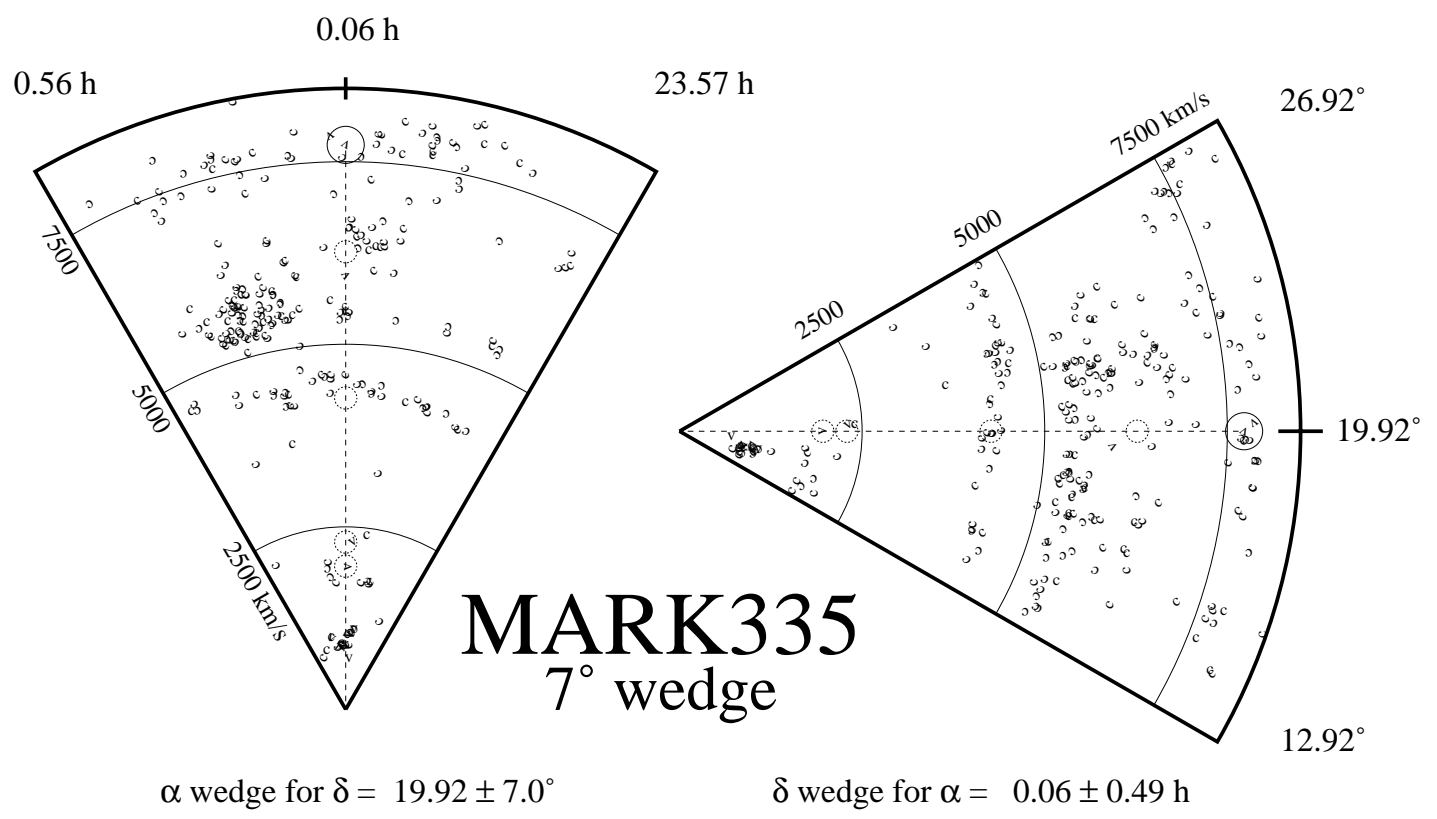

Fig. 29. - Right ascension $(\alpha)$ and declination $(\delta)$ pie diagram for the Markarian 335 sightline.

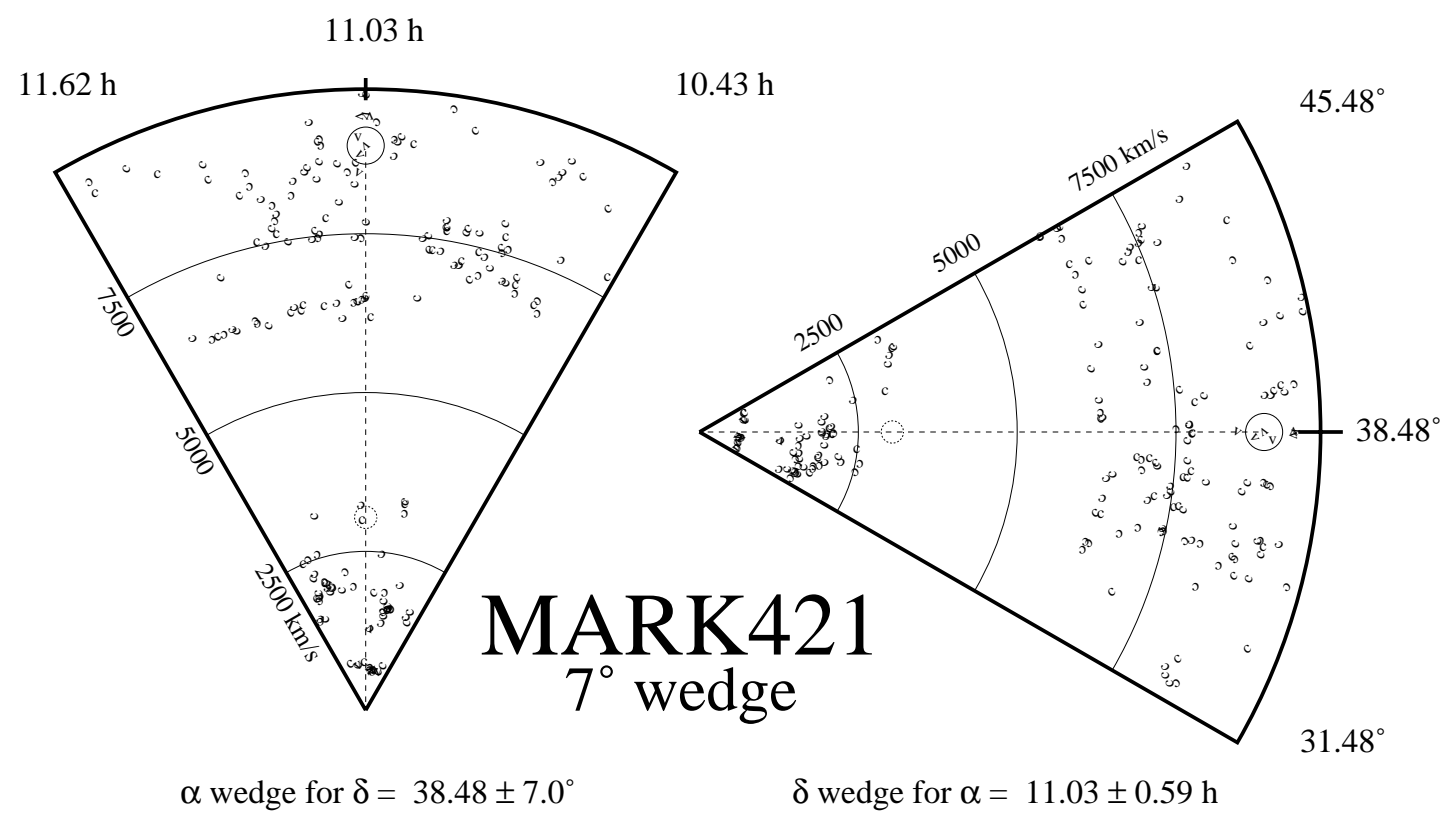

Fig. 30.- Right ascension $(\alpha)$ and declination $(\delta)$ pie diagram for the Markarian 421 sightline. 


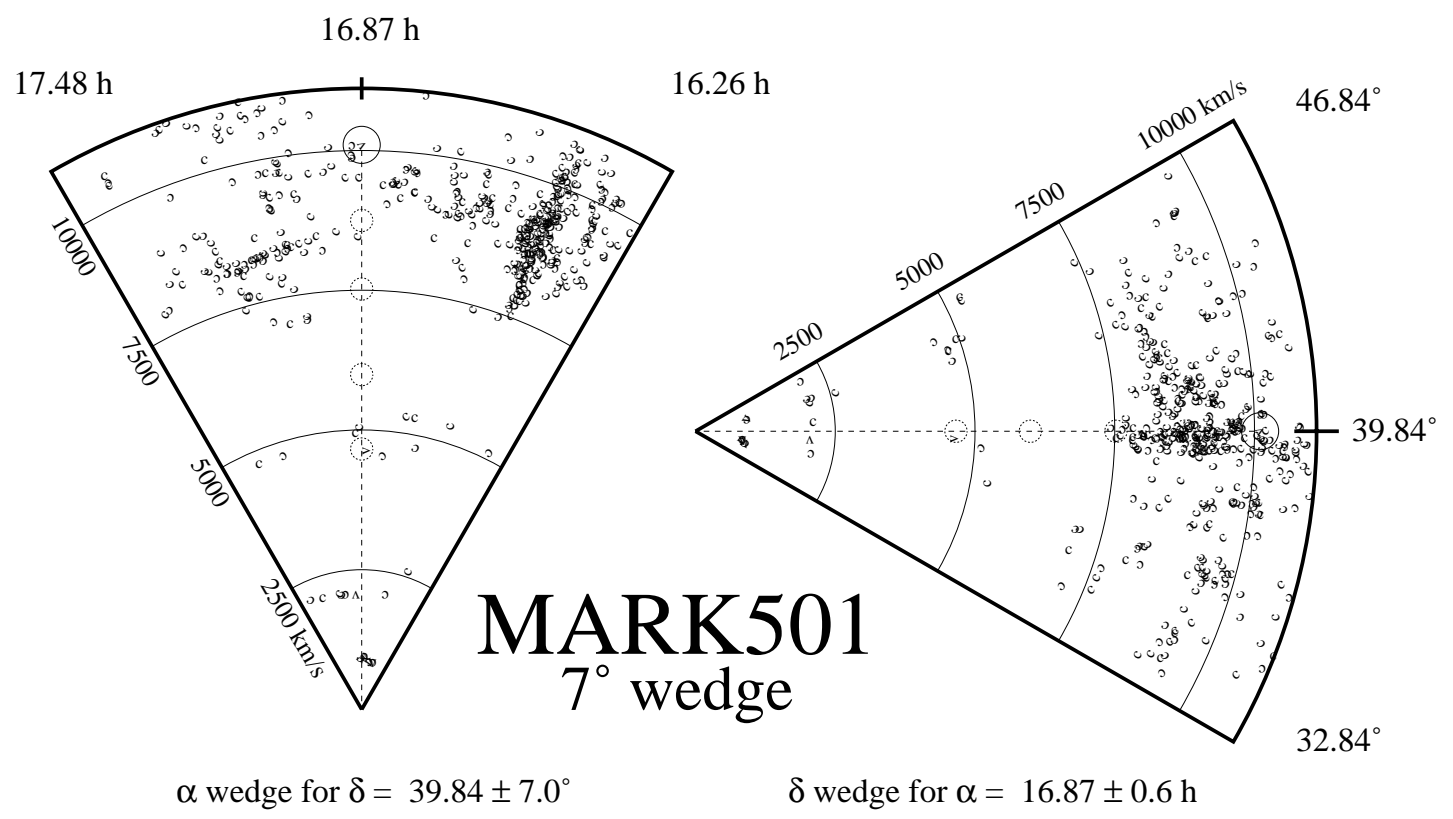

Fig. 31. - Right ascension $(\alpha)$ and declination $(\delta)$ pie diagram for the Markarian 501 sightline.

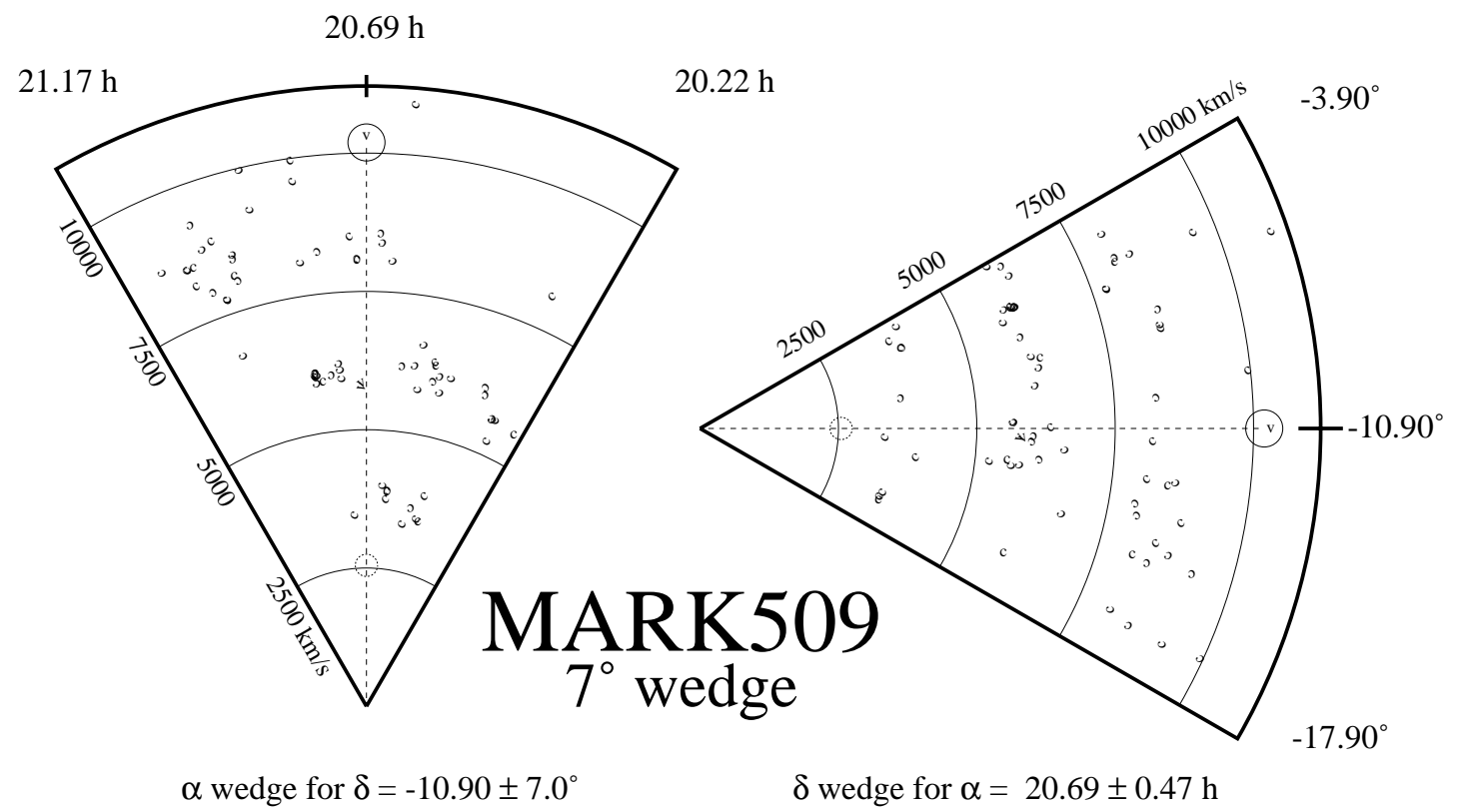

Fig. 32.- Right ascension $(\alpha)$ and declination $(\delta)$ pie diagram for the Markarian 509 sightline. 


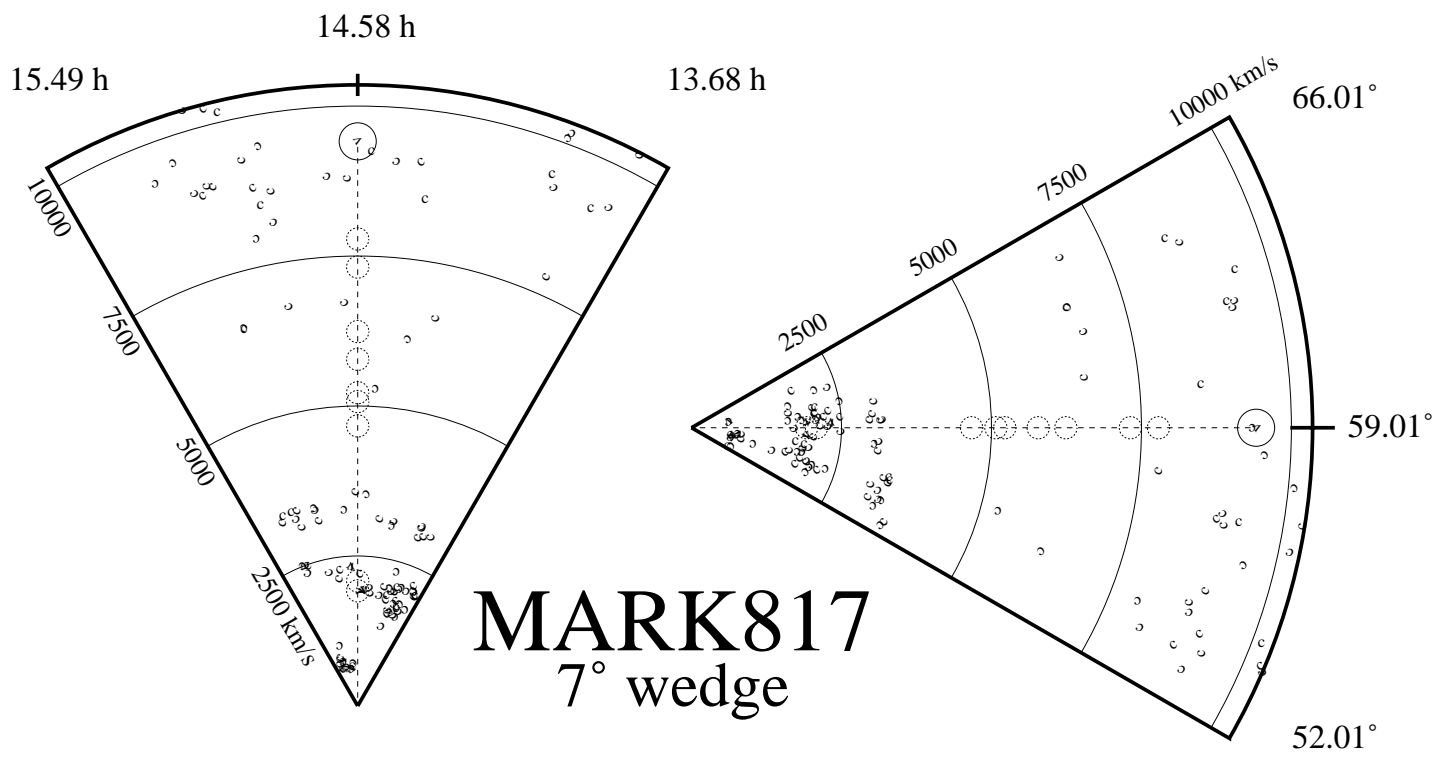

$\alpha$ wedge for $\delta=59.01 \pm 7.0^{\circ} \quad \delta$ wedge for $\alpha=14.58 \pm 0.9 \mathrm{~h}$

Fig. 33.- Right ascension $(\alpha)$ and declination $(\delta)$ pie diagram for the Markarian 817 sightline.

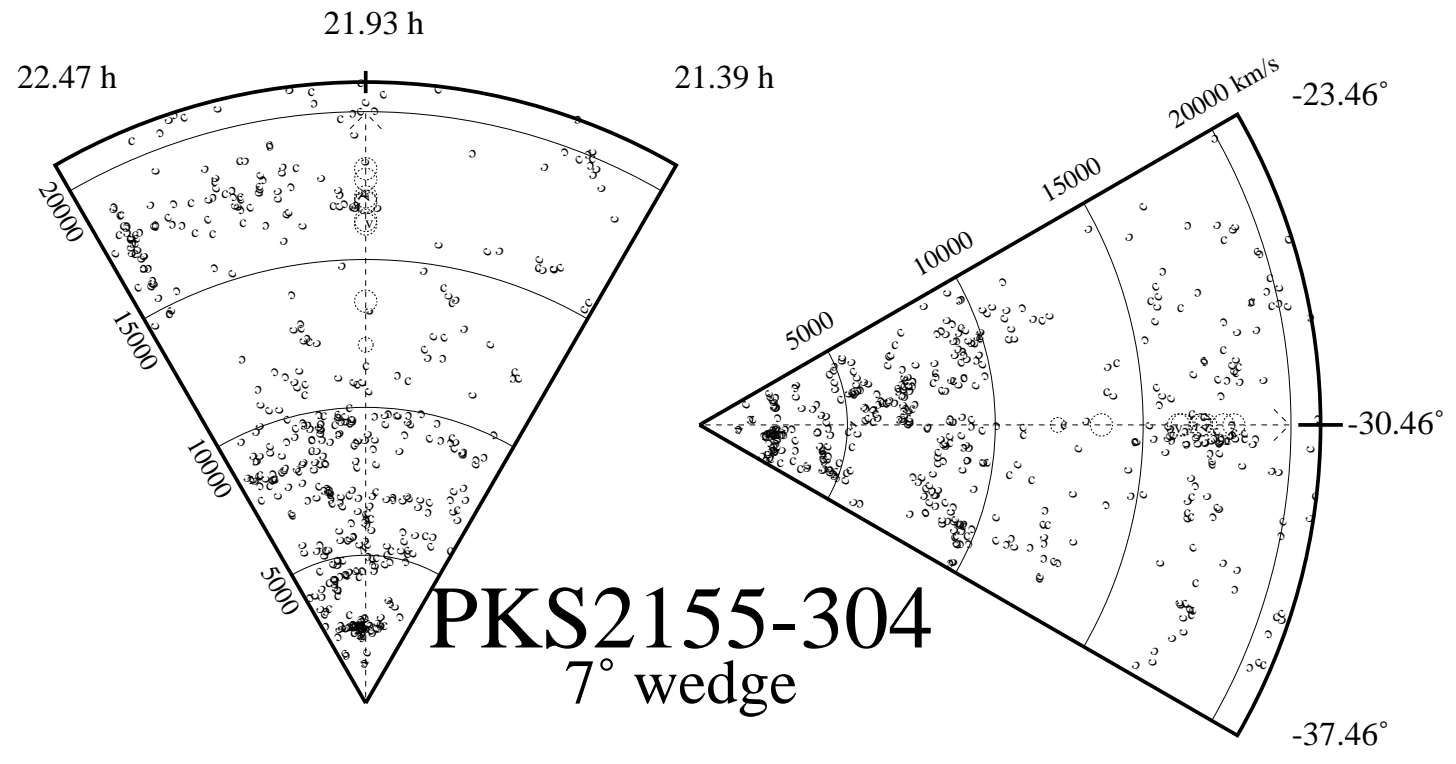

$\alpha$ wedge for $\delta=-30.46 \pm 7.0^{\circ}$

$\delta$ wedge for $\alpha=21.93 \pm 0.54 \mathrm{~h}$

Fig. 34.- Right ascension $(\alpha)$ and declination $(\delta)$ pie diagram for the PKS2155-304 sightline. The arrow indicates that PKS 2155-304 lies beyond the extent of the pie diagram at $z=0.1165$. 


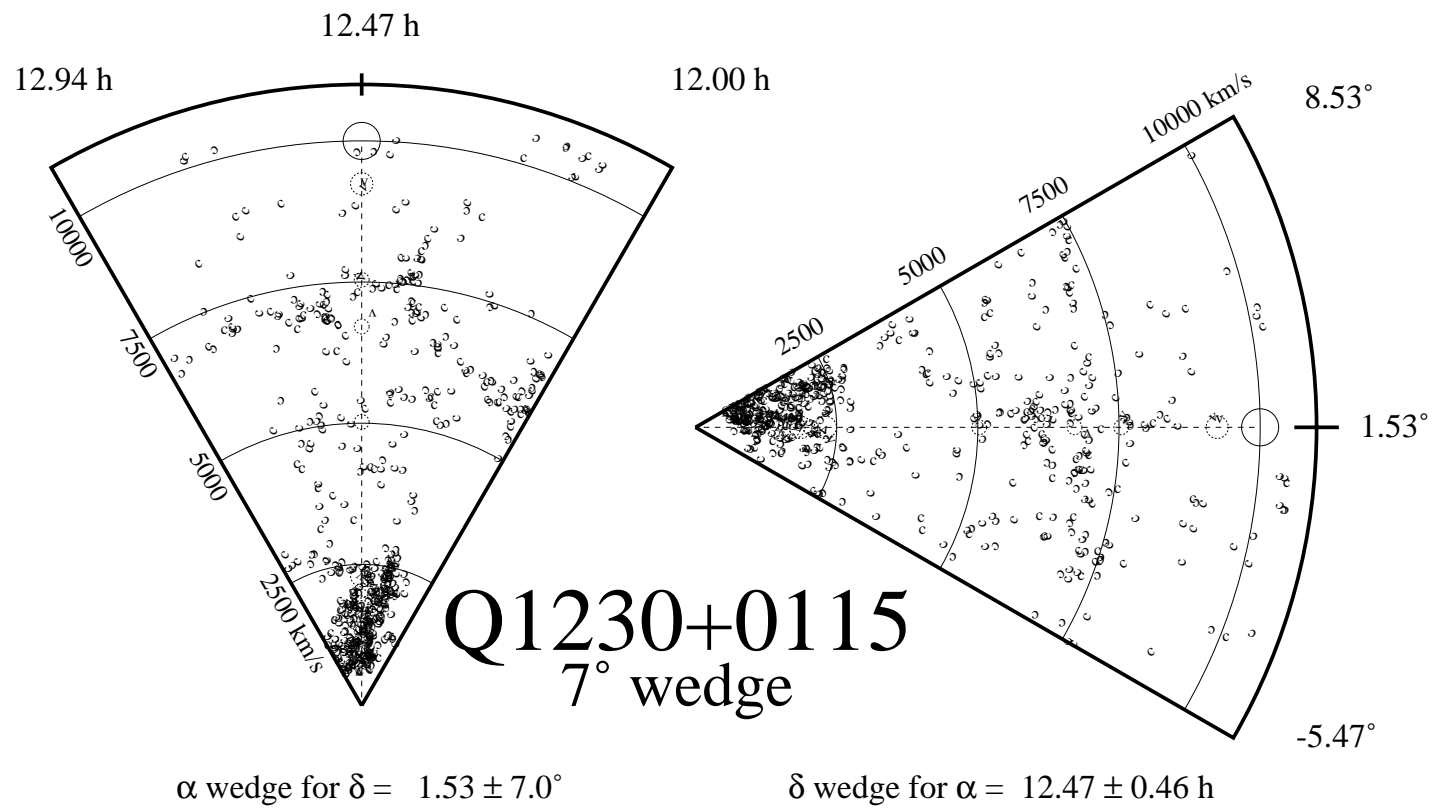

Fig. 35. - Right ascension $(\alpha)$ and declination $(\delta)$ pie diagram for the Q $1230+0115$ sightline. 
Table 10. Nearest three galaxies to each Ly $\alpha$ absorber.

\begin{tabular}{|c|c|c|c|c|c|c|c|}
\hline $\begin{array}{l}\operatorname{Abs}^{\mathrm{a}} \\
\text { Type } \\
(1)\end{array}$ & $\begin{array}{c}\text { Abs Vel } \\
\left(\mathrm{km} \mathrm{s}^{-1}\right) \\
(2)\end{array}$ & $\begin{array}{c}\text { Nearest } \\
\text { Galaxy } \\
(3)\end{array}$ & $\begin{array}{c}D_{\perp}^{\mathrm{b}} \\
\left(h_{70}^{-1} \mathrm{Mpc}\right) \\
(4)\end{array}$ & $\begin{array}{c}\text { Gal Vel } \\
\left(\mathrm{km} \mathrm{s}^{-1}\right) \\
(5)\end{array}$ & $\begin{array}{c}D_{c z}{ }^{\mathrm{c}} \\
\left(h_{70}^{-1} \mathrm{Mpc}\right) \\
(6)\end{array}$ & $\begin{array}{c}D_{\text {tot }} \mathrm{d}^{-} \\
\left(h_{70}^{-1} \mathrm{Mpc}\right) \\
(7)\end{array}$ & $\begin{array}{l}m_{B} \\
(8)\end{array}$ \\
\hline \multicolumn{8}{|c|}{$3 \mathrm{C} 273$} \\
\hline $\mathrm{D}$ & 1012 & $12285+0157$ & 0.170 & 1105 & 0.000 & 0.170 & 15.6 \\
\hline $\mathrm{D}$ & 1012 & $\mathrm{~A} 1225+0152$ & 0.182 & 1298 & 0.000 & 0.182 & 16.3 \\
\hline $\mathrm{D}$ & 1012 & $\mathrm{~A} 1224+0146$ & 0.232 & 1275 & 0.000 & 0.232 & $\ldots$ \\
\hline $\mathrm{D}$ & 1583 & $\mathrm{~A} 1225+0152$ & 0.182 & 1298 & 0.000 & 0.182 & 16.3 \\
\hline $\mathrm{D}$ & 1583 & $12277+0254$ & 0.260 & 1635 & 0.000 & 0.260 & 15.3 \\
\hline $\mathrm{D}$ & 1583 & $\mathrm{~A} 1224+0146$ & 0.232 & 1275 & 0.121 & 0.261 & $\cdots$ \\
\hline $\mathrm{P}$ & 2196 & $\mathrm{~A} 1225+0258$ & 0.396 & 2329 & 0.000 & 0.396 & 16.3 \\
\hline $\mathrm{P}$ & 2196 & $12270+0107$ & 0.677 & 2234 & 0.000 & 0.677 & 17.0 \\
\hline $\mathrm{P}$ & 2196 & $\mathrm{~A} 1228+0116$ & 0.678 & 2289 & 0.000 & 0.678 & 18.3 \\
\hline $\mathrm{D}$ & 2287 & $\mathrm{~A} 1225+0258$ & 0.396 & 2329 & 0.000 & 0.396 & 16.3 \\
\hline $\mathrm{D}$ & 2287 & $12270+0107$ & 0.677 & 2234 & 0.000 & 0.677 & 17.0 \\
\hline $\mathrm{D}$ & 2287 & $\mathrm{~A} 1228+0116$ & 0.678 & 2289 & 0.000 & 0.678 & 18.3 \\
\hline $\mathrm{P}$ & 4691 & $12303+0334$ & 1.932 & 4985 & 0.000 & 1.932 & 15.3 \\
\hline $\mathrm{P}$ & 4691 & $\mathrm{~A} 1232+0317$ & 1.962 & 4605 & 0.000 & 1.962 & 16.5 \\
\hline $\mathrm{P}$ & 4691 & N4496B & 2.244 & 4546 & 0.000 & 2.244 & 14.0 \\
\hline $\mathrm{D}$ & 7870 & $\mathrm{~A} 1228+0205$ & 0.944 & 7593 & 0.000 & 0.944 & 18.5 \\
\hline $\mathrm{D}$ & 7870 & $\mathrm{~A} 1228+0155$ & 1.127 & 7587 & 0.000 & 1.127 & 18.8 \\
\hline $\mathrm{D}$ & 7870 & $\mathrm{~A} 1228+0147$ & 1.432 & 7611 & 0.000 & 1.432 & 17.5 \\
\hline $\mathrm{D}$ & 8829 & $\mathrm{~A} 1226+0211$ & 0.401 & 8807 & 0.000 & 0.401 & 18.1 \\
\hline $\mathrm{D}$ & 8829 & $\mathrm{~A} 1227+0246 \mathrm{~A}$ & 1.046 & 9059 & 0.000 & 1.046 & 17.6 \\
\hline $\mathrm{D}$ & 8829 & $\mathrm{~A} 1227+0246 \mathrm{~B}$ & 1.065 & 9164 & 0.484 & 1.170 & 19.1 \\
\hline $\mathrm{D}$ & 9830 & $\mathrm{~A} 1225+0223$ & 0.394 & 9752 & 0.000 & 0.394 & 17.3 \\
\hline $\mathrm{D}$ & 9830 & $\mathrm{~A} 1227+0144$ & 1.582 & 9281 & 3.572 & 3.907 & 17.7 \\
\hline $\mathrm{D}$ & 9830 & $\mathrm{~A} 1228+0146 \mathrm{~A}$ & 1.515 & 9176 & 5.072 & 5.293 & 19.1 \\
\hline $\mathrm{P}$ & 12335 & $\mathrm{~A} 1221+0122$ & 4.515 & 11841 & 2.842 & 5.334 & 18.9 \\
\hline $\mathrm{P}$ & 12335 & $\mathrm{~A} 1224+0159$ & 1.957 & 13115 & 6.837 & 7.112 & 18.6 \\
\hline $\mathrm{P}$ & 12335 & $12316+0045 \mathrm{E}$ & 6.447 & 12887 & 3.477 & 7.325 & 15.5 \\
\hline $\mathrm{P}$ & 12587 & $\mathrm{~A} 1224+0159$ & 1.957 & 13115 & 3.235 & 3.781 & 18.6 \\
\hline $\mathrm{P}$ & 12587 & $12316+0045 \mathrm{E}$ & 6.447 & 12887 & 0.000 & 6.447 & 15.5 \\
\hline
\end{tabular}


Table 10-Continued

\begin{tabular}{|c|c|c|c|c|c|c|c|}
\hline $\begin{array}{l}\operatorname{Abs}^{\mathrm{a}} \\
\text { Type } \\
(1)\end{array}$ & $\begin{array}{c}\text { Abs Vel } \\
\left(\mathrm{km} \mathrm{s}^{-1}\right) \\
(2)\end{array}$ & $\begin{array}{c}\text { Nearest } \\
\text { Galaxy } \\
(3)\end{array}$ & $\begin{array}{c}D_{\perp}{ }^{\mathrm{b}} \\
\left(h_{70}^{-1} \mathrm{Mpc}\right) \\
(4)\end{array}$ & $\begin{array}{c}\text { Gal Vel } \\
\left(\mathrm{km} \mathrm{s}^{-1}\right) \\
(5)\end{array}$ & $\begin{array}{c}D_{c z}{ }^{\mathrm{c}} \\
\left(h_{70}^{-1} \mathrm{Mpc}\right) \\
(6)\end{array}$ & $\begin{array}{c}D_{\text {tot }}{ }^{\mathrm{d}} \\
\left(h_{70}^{-1} \mathrm{Mpc}\right) \\
(7)\end{array}$ & $\begin{array}{l}m_{B} \\
(8)\end{array}$ \\
\hline $\mathrm{P}$ & 12587 & $\mathrm{~A} 1222+0207$ & 3.705 & 13268 & 5.395 & 6.544 & 17.2 \\
\hline $\mathrm{P}$ & 13141 & $\mathrm{~A} 1224+0159$ & 1.957 & 13115 & 0.000 & 1.957 & 18.6 \\
\hline $\mathrm{P}$ & 13141 & $\mathrm{~A} 1222+0207$ & 3.705 & 13268 & 0.000 & 3.705 & 17.2 \\
\hline $\mathrm{P}$ & 13141 & $12316+0045 \mathrm{E}$ & 6.447 & 12887 & 0.000 & 6.447 & 15.5 \\
\hline $\mathrm{D}$ & 14688 & $\mathrm{~A} 1224+0230 \mathrm{~B}$ & 1.896 & 14860 & 0.000 & 1.896 & 18.5 \\
\hline $\mathrm{D}$ & 14688 & $\mathrm{~A} 1223+0238$ & 3.407 & 14986 & 0.000 & 3.407 & 17.1 \\
\hline $\mathrm{D}$ & 14688 & $12249+0332$ & 4.637 & 14625 & 0.000 & 4.637 & 15.4 \\
\hline $\mathrm{D}$ & 14984 & $\mathrm{~A} 1224+0230 \mathrm{~B}$ & 1.896 & 14860 & 0.000 & 1.896 & 18.5 \\
\hline $\mathrm{D}$ & 14984 & $\mathrm{~A} 1223+0238$ & 3.407 & 14986 & 0.000 & 3.407 & 17.1 \\
\hline $\mathrm{D}$ & 14984 & $12249+0332$ & 4.637 & 14625 & 0.900 & 4.724 & 15.4 \\
\hline $\mathrm{D}$ & 15238 & $\mathrm{~A} 1224+0230 \mathrm{~B}$ & 1.896 & 14860 & 1.137 & 2.210 & 18.5 \\
\hline $\mathrm{D}$ & 15238 & $\mathrm{~A} 1223+0238$ & 3.407 & 14986 & 0.000 & 3.407 & 17.1 \\
\hline $\mathrm{D}$ & 15238 & $12248+0334$ & 4.810 & 14665 & 3.969 & 6.236 & 15.4 \\
\hline $\mathrm{D}$ & 15927 & $\mathrm{~A} 1223+0238$ & 3.407 & 14986 & 9.196 & 9.807 & 17.1 \\
\hline $\mathrm{D}$ & 15927 & $\mathrm{~A} 1224+0230 \mathrm{~B}$ & 1.896 & 14860 & 10.977 & 11.140 & 18.5 \\
\hline $\mathrm{D}$ & 15927 & $12248+0334$ & 4.810 & 14665 & 13.810 & 14.623 & 15.4 \\
\hline $\mathrm{D}$ & 18270 & $\mathrm{~A} 1229+0222$ & 3.906 & 18889 & 4.522 & 5.975 & 18.9 \\
\hline $\mathrm{D}$ & 18270 & $\mathrm{~A} 1226+0000$ & 10.690 & 18415 & 0.000 & 10.690 & $\ldots$ \\
\hline $\mathrm{D}$ & 18270 & 12285-0204 & 19.433 & 17642 & 5.443 & 20.181 & 15.5 \\
\hline $\mathrm{D}$ & 19031 & $\mathrm{~A} 1229+0222$ & 3.906 & 18889 & 0.000 & 3.906 & 18.9 \\
\hline $\mathrm{D}$ & 19031 & $\mathrm{~A} 1226+0000$ & 10.690 & 18415 & 4.732 & 11.691 & $\ldots$ \\
\hline $\mathrm{D}$ & 19031 & $\mathrm{~A} 1222+0249$ & 5.609 & 20661 & 18.946 & 19.759 & 18.0 \\
\hline $\mathrm{D}$ & 19953 & $\mathrm{~A} 1222+0249$ & 5.609 & 20661 & 5.770 & 8.047 & 18.0 \\
\hline $\mathrm{D}$ & 19953 & $\mathrm{~A} 1229+0222$ & 3.906 & 18889 & 10.948 & 11.624 & 18.9 \\
\hline $\mathrm{D}$ & 19953 & 12168-0020W & 18.639 & 20613 & 4.547 & 19.185 & 16.7 \\
\hline \multicolumn{8}{|c|}{ Arakelian 120} \\
\hline $\mathrm{P}$ & 1846 & $04517+0135$ & 3.017 & 2100 & 0.000 & 3.017 & 15.5 \\
\hline $\mathrm{P}$ & 1846 & 04491-0239 & 3.448 & 2100 & 0.000 & 3.448 & 15.1 \\
\hline $\mathrm{P}$ & 1846 & $\mathrm{~A} 0547+0252$ & 3.963 & 1776 & 0.000 & 3.963 & $\ldots$ \\
\hline $\mathrm{D}$ & 4056 & A0510-0036 & 0.865 & 4284 & 0.000 & 0.865 & \\
\hline
\end{tabular}


Table 10-Continued

\begin{tabular}{|c|c|c|c|c|c|c|c|}
\hline $\begin{array}{l}\text { Abs }^{\mathrm{a}} \\
\text { Type } \\
(1)\end{array}$ & $\begin{array}{c}\text { Abs Vel } \\
\left(\mathrm{km} \mathrm{s}^{-1}\right) \\
(2)\end{array}$ & $\begin{array}{c}\text { Nearest } \\
\text { Galaxy } \\
(3)\end{array}$ & $\begin{array}{c}D_{\perp}{ }^{\mathrm{b}} \\
\left(h_{70}^{-1} \mathrm{Mpc}\right) \\
(4)\end{array}$ & $\begin{array}{c}\text { Gal Vel } \\
\left(\mathrm{km} \mathrm{s}^{-1}\right) \\
(5)\end{array}$ & $\begin{array}{c}D_{c z}{ }^{\mathrm{c}} \\
\left(h_{70}^{-1} \mathrm{Mpc}\right) \\
(6)\end{array}$ & $\begin{array}{c}D_{\mathrm{tot}}{ }^{\mathrm{d}} \\
\left(h_{70}^{-1} \mathrm{Mpc}\right) \\
(7)\end{array}$ & $\begin{array}{l}m_{B} \\
(8)\end{array}$ \\
\hline $\mathrm{D}$ & 4056 & A0510-0037 & 0.864 & 4363 & 0.081 & 0.868 & $\cdots$ \\
\hline $\mathrm{D}$ & 4056 & 05152-0113 & 1.245 & 4505 & 2.104 & 2.445 & 16.5 \\
\hline $\mathrm{D}$ & 6749 & 05071-0018 & 2.837 & 6961 & 0.000 & 2.837 & 15.6 \\
\hline $\mathrm{D}$ & 6749 & 05036-0006 & 4.388 & 7027 & 0.000 & 4.388 & 15.7 \\
\hline $\mathrm{D}$ & 6749 & 05017-0029 & 5.224 & 7031 & 0.000 & 5.224 & 15.7 \\
\hline $\mathrm{P}$ & 7808 & A0509-0045 & 2.319 & 7888 & 0.000 & 2.319 & $\cdots$ \\
\hline $\mathrm{P}$ & 7808 & $\mathrm{~A} 0514+0052$ & 2.012 & 7403 & 1.532 & 2.529 & 16.9 \\
\hline $\mathrm{P}$ & 7808 & 05076-0046 & 3.152 & 7858 & 0.000 & 3.152 & 15.2 \\
\hline $\mathrm{D}$ & 7883 & A0509-0045 & 2.319 & 7888 & 0.000 & 2.319 & $\cdots$ \\
\hline $\mathrm{D}$ & 7883 & 05076-0046 & 3.152 & 7858 & 0.000 & 3.152 & 15.2 \\
\hline $\mathrm{D}$ & 7883 & 05077-0047 & 3.222 & 8115 & 0.000 & 3.222 & 15.7 \\
\hline $\mathrm{D}$ & 7977 & A0509-0045 & 2.319 & 7888 & 0.000 & 2.319 & $\cdots$ \\
\hline $\mathrm{D}$ & 7977 & 05076-0046 & 3.152 & 7858 & 0.000 & 3.152 & 15.2 \\
\hline $\mathrm{D}$ & 7977 & 05077-0047 & 3.222 & 8115 & 0.000 & 3.222 & 15.7 \\
\hline $\mathrm{D}$ & 8037 & A0509-0045 & 2.319 & 7888 & 0.000 & 2.319 & $\cdots$ \\
\hline $\mathrm{D}$ & 8037 & 05076-0046 & 3.152 & 7858 & 0.000 & 3.152 & 15.2 \\
\hline $\mathrm{D}$ & 8037 & 05077-0047 & 3.222 & 8115 & 0.000 & 3.222 & 15.7 \\
\hline \multicolumn{8}{|c|}{ ESO 141-G55 } \\
\hline $\mathrm{P}$ & 9078 & $\mathrm{I} 4815$ & 7.398 & 8441 & 5.048 & 8.956 & 14.4 \\
\hline $\mathrm{P}$ & 9078 & A1901-6129 & 6.871 & 8300 & 7.034 & 9.833 & $\cdots$ \\
\hline $\mathrm{P}$ & 9078 & $\mathrm{I} 4849$ & 9.930 & 9292 & 0.000 & 9.930 & $\cdots$ \\
\hline \multicolumn{8}{|c|}{ Fairall 9} \\
\hline $\mathrm{D}$ & 6255 & A0105-5820 & 3.259 & 5914 & 0.651 & 3.323 & 16.1 \\
\hline $\mathrm{D}$ & 6255 & A0142-6122 & 5.600 & 6529 & 0.000 & 5.600 & 14.4 \\
\hline $\mathrm{D}$ & 6255 & A0147-5618 & 6.767 & 6151 & 0.000 & 6.767 & 13.4 \\
\hline $\mathrm{D}$ & 7111 & A0106-5837 & 4.043 & 7796 & 5.414 & 6.757 & $\cdots$ \\
\hline $\mathrm{D}$ & 7111 & A0142-6122 & 5.600 & 6529 & 4.209 & 7.006 & 14.4 \\
\hline $\mathrm{D}$ & 7111 & A0158-6014 & 8.046 & 6743 & 1.321 & 8.154 & 17.1 \\
\hline
\end{tabular}


Table 10-Continued

\begin{tabular}{|c|c|c|c|c|c|c|c|}
\hline $\begin{array}{l}\operatorname{Abs}^{\mathrm{a}} \\
\text { Type } \\
(1)\end{array}$ & $\begin{array}{c}\text { Abs Vel } \\
\left(\mathrm{km} \mathrm{s}^{-1}\right) \\
(2)\end{array}$ & $\begin{array}{c}\text { Nearest } \\
\text { Galaxy } \\
(3)\end{array}$ & $\begin{array}{c}D_{\perp}^{\mathrm{b}} \\
\left(h_{70}^{-1} \mathrm{Mpc}\right) \\
(4)\end{array}$ & $\begin{array}{c}\text { Gal Vel } \\
\left(\mathrm{km} \mathrm{s}^{-1}\right) \\
(5)\end{array}$ & $\begin{array}{c}D_{c z}{ }^{\mathrm{c}} \\
\left(h_{70}^{-1} \mathrm{Mpc}\right) \\
(6)\end{array}$ & $\begin{array}{c}D_{\text {tot }}{ }^{\mathrm{d}} \\
\left(h_{70}^{-1} \mathrm{Mpc}\right) \\
(7)\end{array}$ & $\begin{array}{l}m_{B} \\
(8)\end{array}$ \\
\hline $\mathrm{D}$ & 9498 & A0114-6153 & 6.536 & 8818 & 5.609 & 8.613 & 14.4 \\
\hline $\mathrm{D}$ & 9498 & A0127-6143 & 5.934 & 8657 & 7.881 & 9.866 & $\ldots$ \\
\hline $\mathrm{D}$ & 9498 & A0116-6146 & 5.973 & 8641 & 8.112 & 10.074 & 16.4 \\
\hline $\mathrm{D}$ & 11649 & A0101-5726 & 8.726 & 11114 & 3.611 & 9.444 & $\ldots$ \\
\hline $\mathrm{D}$ & 11649 & A0130-5554 & 10.189 & 12200 & 3.274 & 10.702 & $\ldots$ \\
\hline $\mathrm{D}$ & 11649 & A0103-5636 & 10.558 & 12200 & 3.252 & 11.047 & $\cdots$ \\
\hline $\mathrm{D}$ & 11929 & A0120-5934 & 1.722 & 12927 & 9.956 & 10.104 & 16.3 \\
\hline $\mathrm{D}$ & 11929 & A0104-5903 & 7.141 & 12745 & 7.224 & 10.158 & 15.4 \\
\hline $\mathrm{D}$ & 11929 & A0130-5554 & 10.189 & 12200 & 0.000 & 10.189 & $\ldots$ \\
\hline $\mathrm{D}$ & 12098 & A0120-5934 & 1.722 & 12927 & 7.538 & 7.732 & 16.3 \\
\hline $\mathrm{D}$ & 12098 & A0104-5903 & 7.141 & 12745 & 4.806 & 8.608 & 15.4 \\
\hline $\mathrm{D}$ & 12098 & A0130-5554 & 10.189 & 12200 & 0.000 & 10.189 & $\cdots$ \\
\hline $\mathrm{D}$ & 12202 & A0120-5934 & 1.722 & 12927 & 6.060 & 6.300 & 16.3 \\
\hline $\mathrm{D}$ & 12202 & A0104-5903 & 7.141 & 12745 & 3.328 & 7.878 & 15.4 \\
\hline $\mathrm{D}$ & 12202 & A0117-6006 & 3.876 & 13095 & 8.428 & 9.276 & 16.8 \\
\hline $\mathrm{P}$ & 12277 & A0120-5934 & 1.722 & 12927 & 4.992 & 5.280 & 16.3 \\
\hline $\mathrm{P}$ & 12277 & A0104-5903 & 7.141 & 12745 & 2.260 & 7.490 & 15.4 \\
\hline $\mathrm{P}$ & 12277 & A0117-6006 & 3.876 & 13095 & 7.360 & 8.318 & 16.8 \\
\hline $\mathrm{D}$ & 12415 & A0120-5934 & 1.722 & 12927 & 3.008 & 3.466 & 16.3 \\
\hline $\mathrm{D}$ & 12415 & A0117-6006 & 3.876 & 13095 & 5.376 & 6.628 & 16.8 \\
\hline $\mathrm{D}$ & 12415 & A0104-5903 & 7.141 & 12745 & 0.276 & 7.146 & 15.4 \\
\hline \multicolumn{8}{|c|}{ H $1821+643$} \\
\hline $\mathrm{P}$ & 5493 & $18079+6341$ & 2.480 & 6058 & 3.740 & 4.487 & 15.5 \\
\hline $\mathrm{P}$ & 5493 & $18102+6402$ & 1.956 & 6114 & 4.554 & 4.956 & 15.1 \\
\hline $\mathrm{P}$ & 5493 & N6493 & 6.073 & 5373 & 0.000 & 6.073 & 15.5 \\
\hline $\mathrm{P}$ & 6123 & Tripp0 & 0.871 & 6262 & 0.000 & 0.871 & $\ldots$ \\
\hline $\mathrm{P}$ & 6123 & $18102+6402$ & 1.956 & 6114 & 0.000 & 1.956 & 15.1 \\
\hline $\mathrm{P}$ & 6123 & $18079+6341$ & 2.480 & 6058 & 0.000 & 2.480 & 15.5 \\
\hline $\mathrm{P}$ & 7208 & Tripp1 & 0.869 & 7207 & 0.000 & 0.869 & 17.4 \\
\hline $\mathrm{P}$ & 7208 & $18197+6340 \mathrm{~W}$ & 1.292 & 7364 & 0.000 & 1.292 & 15.7 \\
\hline
\end{tabular}


Table 10-Continued

\begin{tabular}{|c|c|c|c|c|c|c|c|}
\hline $\begin{array}{l}\operatorname{Abs}^{\mathrm{a}} \\
\text { Type } \\
(1)\end{array}$ & $\begin{array}{c}\text { Abs Vel } \\
\left(\mathrm{km} \mathrm{s}^{-1}\right) \\
(2)\end{array}$ & $\begin{array}{c}\text { Nearest } \\
\text { Galaxy } \\
(3)\end{array}$ & $\begin{array}{c}D_{\perp} \mathrm{b} \\
\left(h_{70}^{-1} \mathrm{Mpc}\right) \\
(4)\end{array}$ & $\begin{array}{c}\text { Gal Vel } \\
\left(\mathrm{km} \mathrm{s}^{-1}\right) \\
(5)\end{array}$ & $\begin{array}{c}D_{c z}{ }^{\mathrm{c}} \\
\left(h_{70}^{-1} \mathrm{Mpc}\right) \\
(6)\end{array}$ & $\begin{array}{c}D_{\text {tot }}{ }^{\mathrm{d}} \\
\left(h_{70}^{-1} \mathrm{Mpc}\right) \\
(7)\end{array}$ & $\begin{array}{l}m_{B} \\
(8)\end{array}$ \\
\hline $\mathrm{P}$ & 7208 & $18194+6340$ & 1.294 & 7365 & 0.000 & 1.294 & 15.0 \\
\hline $\mathrm{D}$ & 7325 & Tripp1 & 0.869 & 7207 & 0.000 & 0.869 & 17.4 \\
\hline $\mathrm{D}$ & 7325 & $18197+6340 \mathrm{~W}$ & 1.292 & 7364 & 0.000 & 1.292 & 15.7 \\
\hline $\mathrm{D}$ & 7325 & $18194+6340$ & 1.294 & 7365 & 0.000 & 1.294 & 15.0 \\
\hline $\mathrm{D}$ & 7538 & Tripp1 & 0.869 & 7207 & 0.447 & 0.978 & 17.4 \\
\hline $\mathrm{D}$ & 7538 & $18197+6340 \mathrm{~W}$ & 1.292 & 7364 & 0.000 & 1.292 & 15.7 \\
\hline $\mathrm{D}$ & 7538 & $18194+6340$ & 1.294 & 7365 & 0.000 & 1.294 & 15.0 \\
\hline $\mathrm{P}$ & 7798 & $18197+6340 \mathrm{E}$ & 1.344 & 7756 & 0.000 & 1.344 & 15.7 \\
\hline $\mathrm{P}$ & 7798 & Tripp2 & 0.309 & 8250 & 2.163 & 2.185 & 20.6 \\
\hline $\mathrm{P}$ & 7798 & $18194+6340$ & 1.294 & 7365 & 1.921 & 2.316 & 15.0 \\
\hline $\mathrm{D}$ & 7853 & $18197+6340 \mathrm{E}$ & 1.344 & 7756 & 0.000 & 1.344 & 15.7 \\
\hline $\mathrm{D}$ & 7853 & Tripp2 & 0.309 & 8250 & 1.384 & 1.418 & 20.6 \\
\hline $\mathrm{D}$ & 7853 & Tripp123 & 0.615 & 8256 & 1.469 & 1.592 & 16.6 \\
\hline $\mathrm{D}$ & 7941 & Tripp2 & 0.309 & 8250 & 0.132 & 0.336 & 20.6 \\
\hline $\mathrm{D}$ & 7941 & Tripp123 & 0.615 & 8256 & 0.216 & 0.652 & 16.6 \\
\hline $\mathrm{D}$ & 7941 & $18197+6340 \mathrm{E}$ & 1.344 & 7756 & 0.000 & 1.344 & 15.7 \\
\hline $\mathrm{P}$ & 9060 & $\mathrm{~A} 1803+6312$ & 5.045 & 8694 & 1.050 & 5.153 & $\cdots$ \\
\hline $\mathrm{P}$ & 9060 & Tripp124 & 0.435 & 8358 & 5.746 & 5.762 & 18.5 \\
\hline $\mathrm{P}$ & 9060 & $18424+6226$ & 6.435 & 8662 & 1.573 & 6.625 & 15.5 \\
\hline $\mathrm{P}$ & 9651 & $18009+6301$ & 6.358 & 9652 & 0.000 & 6.358 & 15.7 \\
\hline $\mathrm{P}$ & 9651 & $17593+6246$ & 6.994 & 9560 & 0.000 & 6.994 & 15.6 \\
\hline $\mathrm{P}$ & 9651 & $\mathrm{~A} 1759+6247$ & 7.020 & 9598 & 0.000 & 7.020 & $\cdots$ \\
\hline $\mathrm{P}$ & 11776 & $\mathrm{~A} 1801+6553$ & 7.777 & 11962 & 0.000 & 7.777 & $\cdots$ \\
\hline $\mathrm{P}$ & 11776 & $17560+6439$ & 7.814 & 11360 & 1.851 & 8.030 & 16.5 \\
\hline $\mathrm{P}$ & 11776 & $18421+6358$ & 6.942 & 12369 & 4.044 & 8.034 & 15.7 \\
\hline $\mathrm{D}$ & 12323 & $18421+6358$ & 6.942 & 12369 & 0.000 & 6.942 & 15.7 \\
\hline $\mathrm{D}$ & 12323 & $\mathrm{~A} 1801+6553$ & 7.777 & 11962 & 1.061 & 7.849 & $\ldots$ \\
\hline $\mathrm{D}$ & 12323 & $17583+6243$ & 9.828 & 12881 & 3.411 & 10.403 & 15.7 \\
\hline \multicolumn{8}{|c|}{ Markarian 279} \\
\hline $\mathrm{D}$ & 5233 & $\mathrm{~A} 1310+6745$ & 6.229 & 5994 & 6.349 & 8.894 & 16.0 \\
\hline
\end{tabular}


Table 10-Continued

\begin{tabular}{|c|c|c|c|c|c|c|c|}
\hline $\begin{array}{l}\text { Abs }^{\mathrm{a}} \\
\text { Type } \\
(1)\end{array}$ & $\begin{array}{c}\text { Abs Vel } \\
\left(\mathrm{km} \mathrm{s}^{-1}\right) \\
(2)\end{array}$ & $\begin{array}{c}\text { Nearest } \\
\text { Galaxy } \\
(3)\end{array}$ & $\begin{array}{c}D_{\perp}^{\mathrm{b}} \\
\left(h_{70}^{-1} \mathrm{Mpc}\right) \\
(4)\end{array}$ & $\begin{array}{c}\text { Gal Vel } \\
\left(\mathrm{km} \mathrm{s}^{-1}\right) \\
(5)\end{array}$ & $\begin{array}{c}D_{c z}{ }^{\mathrm{c}} \\
\left(h_{70}^{-1} \mathrm{Mpc}\right) \\
(6)\end{array}$ & $\begin{array}{c}D_{\text {tot }^{\mathrm{d}}} \\
\left(h_{70}^{-1} \mathrm{Mpc}\right) \\
(7)\end{array}$ & $\begin{array}{l}m_{B} \\
(8)\end{array}$ \\
\hline $\mathrm{D}$ & 5233 & $14010+6943$ & 1.277 & 6293 & 10.838 & 10.913 & 15.5 \\
\hline $\mathrm{D}$ & 5233 & A1325+5945 & 12.321 & 4882 & 1.836 & 12.457 & $\cdots$ \\
\hline $\mathrm{P}$ & 5473 & $\mathrm{~A} 1310+6745$ & 6.229 & 5994 & 2.922 & 6.880 & 16.0 \\
\hline $\mathrm{P}$ & 5473 & $14010+6943$ & 1.277 & 6293 & 7.411 & 7.520 & 15.5 \\
\hline $\mathrm{P}$ & 5473 & $\mathrm{~A} 1401+7005$ & 1.532 & 6430 & 9.364 & 9.489 & 16.3 \\
\hline $\mathrm{P}$ & 5618 & $14010+6943$ & 1.277 & 6293 & 5.343 & 5.493 & 15.5 \\
\hline $\mathrm{P}$ & 5618 & $\mathrm{~A} 1310+6745$ & 6.229 & 5994 & 0.853 & 6.287 & 16.0 \\
\hline $\mathrm{P}$ & 5618 & $\mathrm{~A} 1401+7005$ & 1.532 & 6430 & 7.296 & 7.455 & 16.3 \\
\hline $\mathrm{D}$ & 6359 & $14010+6943$ & 1.277 & 6293 & 0.000 & 1.277 & 15.5 \\
\hline $\mathrm{D}$ & 6359 & $\mathrm{~A} 1401+7005$ & 1.532 & 6430 & 0.000 & 1.532 & 16.3 \\
\hline $\mathrm{D}$ & 6359 & $\mathrm{~A} 1310+6745$ & 6.229 & 5994 & 1.169 & 6.338 & 16.0 \\
\hline $\mathrm{D}$ & 6432 & $14010+6943$ & 1.277 & 6293 & 0.000 & 1.277 & 15.5 \\
\hline $\mathrm{D}$ & 6432 & $\mathrm{~A} 1401+7005$ & 1.532 & 6430 & 0.000 & 1.532 & 16.3 \\
\hline $\mathrm{D}$ & 6432 & $\mathrm{~A} 1310+6745$ & 6.229 & 5994 & 2.210 & 6.609 & 16.0 \\
\hline $\mathrm{D}$ & 6913 & $\mathrm{~A} 1401+7005$ & 1.532 & 6430 & 2.631 & 3.045 & 16.3 \\
\hline $\mathrm{D}$ & 6913 & $14010+6943$ & 1.277 & 6293 & 4.584 & 4.759 & 15.5 \\
\hline $\mathrm{D}$ & 6913 & $13300+7256$ & 6.835 & 7187 & 0.000 & 6.835 & 15.3 \\
\hline $\mathrm{D}$ & 7767 & N5607 & 6.004 & 7595 & 0.000 & 6.004 & 13.9 \\
\hline $\mathrm{D}$ & 7767 & $14189+7246$ & 7.178 & 7434 & 0.717 & 7.214 & 15.4 \\
\hline $\mathrm{D}$ & 7767 & $\mathrm{I} 1046$ & 7.904 & 7948 & 0.000 & 7.904 & 14.7 \\
\hline $\mathrm{D}$ & 7845 & N5607 & 6.004 & 7595 & 0.000 & 6.004 & 13.9 \\
\hline $\mathrm{D}$ & 7845 & $14189+7246$ & 7.178 & 7434 & 1.834 & 7.409 & 15.4 \\
\hline $\mathrm{D}$ & 7845 & I1046 & 7.904 & 7948 & 0.000 & 7.904 & 14.7 \\
\hline \multicolumn{8}{|c|}{ Markarian 290} \\
\hline $\mathrm{P}$ & 4207 & $15436+5732$ & 1.305 & 3957 & 0.000 & 1.305 & 16.5 \\
\hline $\mathrm{P}$ & 4207 & $\mathrm{~A} 1542+5722$ & 1.360 & 4287 & 0.000 & 1.360 & 16.0 \\
\hline $\mathrm{P}$ & 4207 & $15554+5851$ & 2.892 & 4115 & 0.000 & 2.892 & 17.0 \\
\hline $\mathrm{D}$ & 4651 & A1542+5722 & 1.360 & 4287 & 0.939 & 1.652 & 16.0 \\
\hline $\mathrm{D}$ & 4651 & $15592+5832$ & 3.411 & 4223 & 1.934 & 3.921 & 14.8 \\
\hline $\mathrm{D}$ & 4651 & $\mathrm{~A} 1543+5951$ & 2.682 & 5156 & 2.870 & 3.928 & 18.5 \\
\hline
\end{tabular}


Table 10-Continued

\begin{tabular}{|c|c|c|c|c|c|c|c|}
\hline $\begin{array}{l}\mathrm{Abs}^{\mathrm{a}} \\
\text { Type } \\
(1)\end{array}$ & $\begin{array}{c}\text { Abs Vel } \\
\left(\mathrm{km} \mathrm{s}^{-1}\right) \\
(2)\end{array}$ & $\begin{array}{c}\text { Nearest } \\
\text { Galaxy } \\
(3)\end{array}$ & $\begin{array}{c}D_{\perp}^{\mathrm{b}} \\
\left(h_{70}^{-1} \mathrm{Mpc}\right) \\
(4)\end{array}$ & $\begin{array}{c}\text { Gal Vel } \\
\left(\mathrm{km} \mathrm{s}^{-1}\right) \\
(5)\end{array}$ & $\begin{array}{c}D_{c z}{ }^{\mathrm{c}} \\
\left(h_{70}^{-1} \mathrm{Mpc}\right) \\
(6)\end{array}$ & $\begin{array}{c}D_{\text {tot }}{ }^{\mathrm{d}} \\
\left(h_{70}^{-1} \mathrm{Mpc}\right) \\
(7)\end{array}$ & $\begin{array}{l}m_{B} \\
(8)\end{array}$ \\
\hline $\mathrm{P}$ & 4939 & A1543+5951 & 2.682 & 5156 & 0.000 & 2.682 & 18.5 \\
\hline $\mathrm{P}$ & 4939 & $\mathrm{~A} 1542+5722$ & 1.360 & 4287 & 5.050 & 5.230 & 16.0 \\
\hline $\mathrm{P}$ & 4939 & $\mathrm{~A} 1602+6000$ & 4.393 & 4325 & 4.648 & 6.396 & $\ldots$ \\
\hline $\mathrm{D}$ & 7071 & $\mathrm{~A} 1505+6310$ & 10.228 & 6584 & 3.231 & 10.726 & $\cdots$ \\
\hline $\mathrm{D}$ & 7071 & I1100 & 10.188 & 6561 & 3.557 & 10.791 & 14.1 \\
\hline $\mathrm{D}$ & 7071 & I1049 & 13.595 & 6728 & 1.582 & 13.687 & 14.8 \\
\hline $\mathrm{D}$ & 7349 & $\mathrm{~A} 1505+6310$ & 10.228 & 6584 & 7.205 & 12.511 & $\cdots$ \\
\hline $\mathrm{D}$ & 7349 & I1100 & 10.188 & 6561 & 7.531 & 12.669 & 14.1 \\
\hline $\mathrm{D}$ & 7349 & $\mathrm{~A} 1457+5403$ & 13.074 & 7974 & 3.886 & 13.639 & 16.0 \\
\hline $\mathrm{P}$ & 7431 & $\mathrm{~A} 1505+6310$ & 10.228 & 6584 & 8.380 & 13.223 & $\cdots$ \\
\hline $\mathrm{P}$ & 7431 & $\mathrm{~A} 1457+5403$ & 13.074 & 7974 & 2.710 & 13.352 & 16.0 \\
\hline $\mathrm{P}$ & 7431 & I1100 & 10.188 & 6561 & 8.706 & 13.401 & 14.1 \\
\hline \multicolumn{8}{|c|}{ Markarian 335} \\
\hline $\mathrm{D}$ & 1961 & $00025+1956$ & 0.096 & 1950 & 0.000 & 0.096 & 16.0 \\
\hline $\mathrm{D}$ & 1961 & N7817 & 0.446 & 2310 & 0.692 & 0.823 & 12.7 \\
\hline $\mathrm{D}$ & 1961 & $23533+1810$ & 1.309 & 1738 & 0.000 & 1.309 & 17.0 \\
\hline $\mathrm{D}$ & 2291 & N7817 & 0.446 & 2310 & 0.000 & 0.446 & 12.7 \\
\hline $\mathrm{D}$ & 2291 & $00025+1956$ & 0.096 & 1950 & 0.589 & 0.597 & 16.0 \\
\hline $\mathrm{D}$ & 2291 & N7798 & 1.022 & 2403 & 0.000 & 1.022 & 12.7 \\
\hline $\mathrm{D}$ & 4265 & $00008+2150$ & 2.245 & 4455 & 0.000 & 2.245 & 16.0 \\
\hline $\mathrm{D}$ & 4265 & $00017+2219$ & 2.696 & 4444 & 0.000 & 2.696 & 15.4 \\
\hline $\mathrm{D}$ & 4265 & $\mathrm{~A} 0014+2031$ & 2.879 & 4366 & 0.000 & 2.879 & 15.9 \\
\hline $\mathrm{D}$ & 6266 & $00036+1928$ & 0.692 & 5936 & 0.433 & 0.816 & 17.0 \\
\hline $\mathrm{D}$ & 6266 & N 15 & 2.457 & 6332 & 0.000 & 2.457 & 14.9 \\
\hline $\mathrm{D}$ & 6266 & $\mathrm{~A} 0002+1801$ & 3.033 & 6315 & 0.000 & 3.033 & 17.2 \\
\hline \multicolumn{8}{|c|}{ Markarian 421} \\
\hline $\mathrm{D}$ & 3035 & $\mathrm{~A} 1102+4120$ & 2.142 & 2988 & 0.000 & 2.142 & 17.5 \\
\hline $\mathrm{D}$ & 3035 & $11223+3554$ & 3.105 & 2570 & 2.490 & 3.980 & 15.3 \\
\hline
\end{tabular}


Table 10-Continued

\begin{tabular}{|c|c|c|c|c|c|c|c|}
\hline $\begin{array}{l}\text { Abs }^{\mathrm{a}} \\
\text { Type } \\
(1)\end{array}$ & $\begin{array}{c}\text { Abs Vel } \\
\left(\mathrm{km} \mathrm{s}^{-1}\right) \\
(2)\end{array}$ & $\begin{array}{c}\text { Nearest } \\
\text { Galaxy } \\
(3)\end{array}$ & $\begin{array}{c}D_{\perp}^{\mathrm{b}} \\
\left(h_{70}^{-1} \mathrm{Mpc}\right) \\
(4)\end{array}$ & $\begin{array}{c}\text { Gal Vel } \\
\left(\mathrm{km} \mathrm{s}^{-1}\right) \\
(5)\end{array}$ & $\begin{array}{c}D_{c z}{ }^{-1} \\
\left(h_{70}^{-1} \mathrm{Mpc}\right) \\
(6)\end{array}$ & $\begin{array}{c}D_{\text {tot }}{ }^{\mathrm{d}} \\
\left(h_{70}^{-1} \mathrm{Mpc}\right) \\
(7)\end{array}$ & $\begin{array}{l}m_{B} \\
(8)\end{array}$ \\
\hline $\mathrm{D}$ & 3035 & $11032+4336$ & 4.119 & 3224 & 0.000 & 4.119 & 14.6 \\
\hline \multicolumn{8}{|c|}{ Markarian 501} \\
\hline $\mathrm{D}$ & 4659 & $16510+3927$ & 0.517 & 4625 & 0.000 & 0.517 & 15.0 \\
\hline $\mathrm{D}$ & 4659 & $16381+4322$ & 5.249 & 4763 & 0.000 & 5.249 & 15.6 \\
\hline $\mathrm{D}$ & 4659 & $\mathrm{~A} 1646+4437$ & 5.559 & 4551 & 0.000 & 5.559 & 16.7 \\
\hline $\mathrm{D}$ & 5990 & $16310+3727$ & 6.300 & 5309 & 5.707 & 8.501 & 15.2 \\
\hline $\mathrm{D}$ & 5990 & $16416+4556$ & 8.433 & 5301 & 6.030 & 10.367 & 15.3 \\
\hline $\mathrm{D}$ & 5990 & $\mathrm{I} 1226$ & 8.786 & 5298 & 6.114 & 10.703 & 15.4 \\
\hline $\mathrm{D}$ & 7521 & $17048+4107 \mathrm{E}$ & 5.298 & 7852 & 0.313 & 5.307 & 15.6 \\
\hline $\mathrm{D}$ & 7521 & $\mathrm{~A} 1709+3941 \mathrm{~B}$ & 6.167 & 7600 & 0.000 & 6.167 & $\ldots$ \\
\hline $\mathrm{D}$ & 7521 & $16360+4059$ & 6.500 & 7908 & 1.051 & 6.584 & 16.0 \\
\hline $\mathrm{D}$ & 8748 & $16572+4012$ & 2.215 & 8714 & 0.000 & 2.215 & 14.9 \\
\hline $\mathrm{D}$ & 8748 & $16482+4031$ & 2.281 & 8956 & 0.000 & 2.281 & 15.7 \\
\hline $\mathrm{D}$ & 8748 & $16558+4049$ & 2.559 & 8625 & 0.000 & 2.559 & 15.4 \\
\hline \multicolumn{8}{|c|}{ Markarian 509} \\
\hline $\mathrm{D}$ & 2548 & A2028-1821 & 4.662 & 2314 & 0.000 & 4.662 & $\ldots$ \\
\hline $\mathrm{D}$ & 2548 & A2115-0914 & 5.148 & 2402 & 0.000 & 5.148 & $\ldots$ \\
\hline $\mathrm{D}$ & 2548 & A2107-0354 & 5.268 & 2229 & 0.718 & 5.317 & 14.5 \\
\hline \multicolumn{8}{|c|}{ Markarian 817} \\
\hline $\mathrm{D}$ & 1918 & $14332+5934$ & 0.282 & 1920 & 0.000 & 0.282 & 15.5 \\
\hline $\mathrm{D}$ & 1918 & N5667 & 0.495 & 1943 & 0.000 & 0.495 & 13.1 \\
\hline $\mathrm{D}$ & 1918 & N5678 & 0.496 & 1921 & 0.000 & 0.496 & 12.3 \\
\hline $\mathrm{D}$ & 2082 & $14332+5934$ & 0.282 & 1920 & 0.000 & 0.282 & 15.5 \\
\hline $\mathrm{D}$ & 2082 & $\mathrm{~A} 1439+5930$ & 0.464 & 2331 & 0.000 & 0.464 & 17.0 \\
\hline $\mathrm{D}$ & 2082 & $14399+5932$ & 0.467 & 2325 & 0.000 & 0.467 & 17.0 \\
\hline $\mathrm{D}$ & 4668 & $14293+5528$ & 4.783 & 5291 & 4.459 & 6.539 & 15.6 \\
\hline
\end{tabular}


Table 10-Continued

\begin{tabular}{|c|c|c|c|c|c|c|c|}
\hline $\begin{array}{l}\operatorname{Abs}^{\mathrm{a}} \\
\text { Type } \\
(1)\end{array}$ & $\begin{array}{c}\text { Abs Vel } \\
\left(\mathrm{km} \mathrm{s}^{-1}\right) \\
(2)\end{array}$ & $\begin{array}{c}\text { Nearest } \\
\text { Galaxy } \\
(3)\end{array}$ & $\begin{array}{c}D_{\perp}^{\mathrm{b}} \\
\left(h_{70}^{-1} \mathrm{Mpc}\right) \\
(4)\end{array}$ & $\begin{array}{c}\text { Gal Vel } \\
\left(\mathrm{km} \mathrm{s}^{-1}\right) \\
(5)\end{array}$ & $\begin{array}{c}D_{c z}{ }^{\mathrm{c}} \\
\left(h_{70}^{-1} \mathrm{Mpc}\right) \\
(6)\end{array}$ & $\begin{array}{c}D_{\text {tot }^{\mathrm{d}}} \\
\left(h_{70}^{-1} \mathrm{Mpc}\right) \\
(7)\end{array}$ & $\begin{array}{l}m_{B} \\
(8)\end{array}$ \\
\hline $\mathrm{D}$ & 4668 & N5368 & 8.403 & 4642 & 0.000 & 8.403 & 13.8 \\
\hline $\mathrm{D}$ & 4668 & $13454+5549$ & 8.554 & 4660 & 0.000 & 8.554 & 15.5 \\
\hline $\mathrm{D}$ & 5074 & $14293+5528$ & 4.783 & 5291 & 0.000 & 4.783 & 15.6 \\
\hline $\mathrm{D}$ & 5074 & N5368 & 8.403 & 4642 & 2.421 & 8.745 & 13.8 \\
\hline $\mathrm{D}$ & 5074 & $13454+5549$ & 8.554 & 4660 & 2.181 & 8.828 & 15.5 \\
\hline $\mathrm{D}$ & 5221 & $14293+5528$ & 4.783 & 5291 & 0.000 & 4.783 & 15.6 \\
\hline $\mathrm{D}$ & 5221 & N5368 & 8.403 & 4642 & 4.531 & 9.547 & 13.8 \\
\hline $\mathrm{D}$ & 5221 & $13454+5549$ & 8.554 & 4660 & 4.291 & 9.570 & 15.5 \\
\hline $\mathrm{D}$ & 5778 & $14293+5528$ & 4.783 & 5291 & 2.831 & 5.558 & 15.6 \\
\hline $\mathrm{D}$ & 5778 & $\mathrm{~A} 1420+5427$ & 7.590 & 6176 & 1.065 & 7.664 & 18.5 \\
\hline $\mathrm{D}$ & 5778 & $\mathrm{~A} 1414+6042$ & 5.079 & 6595 & 7.241 & 8.845 & 16.0 \\
\hline $\mathrm{D}$ & 6240 & A1414+6042 & 5.079 & 6595 & 0.637 & 5.119 & 16.0 \\
\hline $\mathrm{D}$ & 6240 & I1049 & 5.429 & 6728 & 2.521 & 5.986 & 14.8 \\
\hline $\mathrm{D}$ & 6240 & $\mathrm{~A} 1420+5427$ & 7.590 & 6176 & 0.000 & 7.590 & 18.5 \\
\hline $\mathrm{D}$ & 7316 & I1049 & 5.429 & 6728 & 4.271 & 6.908 & 14.8 \\
\hline $\mathrm{D}$ & 7316 & $\mathrm{~A} 1414+6042$ & 5.079 & 6595 & 6.154 & 7.979 & 16.0 \\
\hline $\mathrm{D}$ & 7316 & $14526+6448$ & 10.338 & 6761 & 4.201 & 11.159 & 15.1 \\
\hline $\mathrm{D}$ & 7784 & $14212+6010$ & 4.435 & 8520 & 6.138 & 7.572 & 15.3 \\
\hline $\mathrm{D}$ & 7784 & $\mathrm{~A} 1351+5746$ & 11.212 & 7817 & 0.000 & 11.212 & $\ldots$ \\
\hline $\mathrm{D}$ & 7784 & A1457+5403 & 11.557 & 7974 & 0.000 & 11.557 & 16.0 \\
\hline \multicolumn{8}{|c|}{ PKS 2155-304 : Combined } \\
\hline $\mathrm{D}$ & 2629 & A2156-3128 & 0.699 & 2786 & 0.000 & 0.699 & 16.7 \\
\hline $\mathrm{D}$ & 2629 & A2157-3142 & 0.796 & 2540 & 0.000 & 0.796 & 15.5 \\
\hline $\mathrm{D}$ & 2629 & A2159-3127 & 0.801 & 2608 & 0.000 & 0.801 & 15.4 \\
\hline $\mathrm{D}$ & 2782 & A2156-3128 & 0.699 & 2786 & 0.000 & 0.699 & 16.7 \\
\hline $\mathrm{D}$ & 2782 & A2157-3142 & 0.796 & 2540 & 0.000 & 0.796 & 15.5 \\
\hline $\mathrm{D}$ & 2782 & A2159-3127 & 0.801 & 2608 & 0.000 & 0.801 & 15.4 \\
\hline $\mathrm{D}$ & 4028 & N7201 & 2.160 & 4452 & 1.729 & 2.767 & 13.8 \\
\hline $\mathrm{D}$ & 4028 & A2159-3307 & 2.921 & 4266 & 0.000 & 2.921 & 14.4 \\
\hline $\mathrm{D}$ & 4028 & N7221 & 2.923 & 4358 & 0.354 & 2.944 & 13.1 \\
\hline
\end{tabular}


Table 10-Continued

\begin{tabular}{|c|c|c|c|c|c|c|c|}
\hline $\begin{array}{l}\mathrm{Abs}^{\mathrm{a}} \\
\text { Type } \\
(1)\end{array}$ & $\begin{array}{c}\text { Abs Vel } \\
\left(\mathrm{km} \mathrm{s}^{-1}\right) \\
(2)\end{array}$ & $\begin{array}{c}\text { Nearest } \\
\text { Galaxy } \\
(3)\end{array}$ & $\begin{array}{c}D_{\perp}{ }^{\mathrm{b}} \\
\left(h_{70}^{-1} \mathrm{Mpc}\right) \\
(4)\end{array}$ & $\begin{array}{c}\text { Gal Vel } \\
\left(\mathrm{km} \mathrm{s}^{-1}\right) \\
(5)\end{array}$ & $\begin{array}{c}D_{c z}{ }^{\mathrm{c}} \\
\left(h_{70}^{-1} \mathrm{Mpc}\right) \\
(6)\end{array}$ & $\begin{array}{c}D_{\mathrm{tot}^{\mathrm{d}}} \\
\left(h_{70}^{-1} \mathrm{Mpc}\right) \\
(7)\end{array}$ & $\begin{array}{l}m_{B} \\
(8)\end{array}$ \\
\hline $\mathrm{P}$ & 4706 & N7201 & 2.160 & 4452 & 0.000 & 2.160 & 13.8 \\
\hline $\mathrm{P}$ & 4706 & A2147-3109 & 2.535 & 5044 & 0.489 & 2.582 & 16.3 \\
\hline $\mathrm{P}$ & 4706 & A $2157-3025$ & 0.332 & 5187 & 2.576 & 2.597 & 14.4 \\
\hline $\mathrm{D}$ & 4948 & A2157-3025 & 0.332 & 5187 & 0.000 & 0.332 & 14.4 \\
\hline $\mathrm{D}$ & 4948 & A2147-3109 & 2.535 & 5044 & 0.000 & 2.535 & 16.3 \\
\hline $\mathrm{D}$ & 4948 & I5139 & 2.636 & 5362 & 1.573 & 3.070 & 13.0 \\
\hline $\mathrm{D}$ & 5010 & A2157-3025 & 0.332 & 5187 & 0.000 & 0.332 & 14.4 \\
\hline $\mathrm{D}$ & 5010 & A2147-3109 & 2.535 & 5044 & 0.000 & 2.535 & 16.3 \\
\hline $\mathrm{D}$ & 5010 & I5139 & 2.636 & 5362 & 0.692 & 2.726 & 13.0 \\
\hline $\mathrm{D}$ & 5115 & A2157-3025 & 0.332 & 5187 & 0.000 & 0.332 & 14.4 \\
\hline $\mathrm{D}$ & 5115 & A2147-3109 & 2.535 & 5044 & 0.000 & 2.535 & 16.3 \\
\hline $\mathrm{D}$ & 5115 & I5139 & 2.636 & 5362 & 0.000 & 2.636 & 13.0 \\
\hline $\mathrm{D}$ & 5615 & A2157-3025 & 0.332 & 5187 & 1.834 & 1.864 & 14.4 \\
\hline $\mathrm{D}$ & 5615 & A2200-2909 & 2.317 & 5658 & 0.000 & 2.317 & $\cdots$ \\
\hline $\mathrm{D}$ & 5615 & A $2200-2900$ & 2.457 & 5614 & 0.000 & 2.457 & 15.3 \\
\hline $\mathrm{D}$ & 5670 & A2200-2909 & 2.317 & 5658 & 0.000 & 2.317 & $\ldots$ \\
\hline $\mathrm{D}$ & 5670 & A2200-2900 & 2.457 & 5614 & 0.000 & 2.457 & 15.3 \\
\hline $\mathrm{D}$ & 5670 & A2157-3025 & 0.332 & 5187 & 2.615 & 2.636 & 14.4 \\
\hline $\mathrm{P}$ & 7720 & A2159-2820 & 4.117 & 7210 & 3.096 & 5.152 & 15.2 \\
\hline $\mathrm{P}$ & 7720 & A2207-2913 & 5.238 & 7324 & 1.517 & 5.453 & 16.6 \\
\hline $\mathrm{P}$ & 7720 & A2206-2801 & 6.078 & 7319 & 1.634 & 6.294 & 14.0 \\
\hline $\mathrm{P}$ & 9717 & A2159-3042 & 1.814 & 9737 & 0.000 & 1.814 & 15.3 \\
\hline $\mathrm{P}$ & 9717 & A2159-3047 & 2.132 & 9454 & 0.000 & 2.132 & 16.2 \\
\hline $\mathrm{P}$ & 9717 & A2153-2928 & 2.777 & 9664 & 0.000 & 2.777 & 16.0 \\
\hline $\mathrm{P}$ & 10099 & A2159-3042 & 1.814 & 9737 & 0.909 & 2.029 & 15.3 \\
\hline $\mathrm{P}$ & 10099 & A2153-2928 & 2.777 & 9664 & 1.968 & 3.403 & 16.0 \\
\hline $\mathrm{P}$ & 10099 & A2201-3212 & 5.247 & 9804 & 0.038 & 5.247 & $\ldots$ \\
\hline $\mathrm{P}$ & 12114 & A2158-3112 & 2.759 & 11509 & 4.390 & 5.185 & 16.5 \\
\hline $\mathrm{P}$ & 12114 & A2158-3108 & 2.565 & 11470 & 4.944 & 5.570 & 16.5 \\
\hline $\mathrm{P}$ & 12114 & A2205-3027 & 6.348 & 12334 & 0.000 & 6.348 & 16.1 \\
\hline $\mathrm{D}$ & 13588 & A2143-2933 & 9.994 & 14030 & 1.769 & 10.150 & 15.6 \\
\hline $\mathrm{D}$ & 13588 & A $2142-2923$ & 10.548 & 13815 & 0.000 & 10.548 & 16.5 \\
\hline
\end{tabular}


Table 10-Continued

\begin{tabular}{|c|c|c|c|c|c|c|c|}
\hline $\begin{array}{l}\text { Abs }^{\mathrm{a}} \\
\text { Type } \\
(1)\end{array}$ & $\begin{array}{c}\text { Abs Vel } \\
\left(\mathrm{km} \mathrm{s}^{-1}\right) \\
(2)\end{array}$ & $\begin{array}{c}\text { Nearest } \\
\text { Galaxy } \\
(3)\end{array}$ & $\begin{array}{c}D_{\perp}^{\mathrm{b}} \\
\left(h_{70}^{-1} \mathrm{Mpc}\right) \\
(4)\end{array}$ & $\begin{array}{c}\text { Gal Vel } \\
\left(\mathrm{km} \mathrm{s}^{-1}\right) \\
(5)\end{array}$ & $\begin{array}{c}D_{c z}{ }^{\mathrm{c}} \\
\left(h_{70}^{-1} \mathrm{Mpc}\right) \\
(6)\end{array}$ & $\begin{array}{c}D_{\mathrm{tot}}^{\mathrm{d}} \\
\left(h_{70}^{-1} \mathrm{Mpc}\right) \\
(7)\end{array}$ & $\begin{array}{l}m_{B} \\
(8)\end{array}$ \\
\hline $\mathrm{D}$ & 13588 & A2139-3020 & 11.257 & 13088 & 3.207 & 11.705 & 16.4 \\
\hline $\mathrm{D}$ & 16200 & A2155-3033A & 0.576 & 16200 & 0.000 & 0.576 & 16.1 \\
\hline $\mathrm{D}$ & 16200 & A2156-3030B & 0.888 & 16785 & 4.060 & 4.156 & $\ldots$ \\
\hline $\mathrm{D}$ & 16200 & A2154-3155 & 6.259 & 16730 & 3.194 & 7.027 & 15.9 \\
\hline $\mathrm{D}$ & 16321 & A2155-3033A & 0.576 & 16200 & 0.000 & 0.576 & 16.1 \\
\hline $\mathrm{D}$ & 16321 & A2156-3030B & 0.888 & 16785 & 2.329 & 2.492 & $\ldots$ \\
\hline $\mathrm{D}$ & 16321 & A2154-3155 & 6.259 & 16730 & 1.462 & 6.428 & 15.9 \\
\hline $\mathrm{D}$ & 16922 & A2155-3033B & 0.441 & 17093 & 0.000 & 0.441 & $\ldots$ \\
\hline $\mathrm{D}$ & 16922 & A2156-3030A & 0.653 & 17156 & 0.000 & 0.653 & $\ldots$ \\
\hline $\mathrm{D}$ & 16922 & A2156-3017 & 0.772 & 17179 & 0.000 & 0.772 & $\ldots$ \\
\hline $\mathrm{D}$ & 16970 & A2155-3033B & 0.441 & 17093 & 0.000 & 0.441 & $\ldots$ \\
\hline $\mathrm{D}$ & 16970 & A2156-3030A & 0.653 & 17156 & 0.000 & 0.653 & $\cdots$ \\
\hline $\mathrm{D}$ & 16970 & A2156-3017 & 0.772 & 17179 & 0.000 & 0.772 & $\ldots$ \\
\hline $\mathrm{D}$ & 17115 & A2155-3033B & 0.441 & 17093 & 0.000 & 0.441 & $\ldots$ \\
\hline $\mathrm{D}$ & 17115 & A2156-3030A & 0.653 & 17156 & 0.000 & 0.653 & $\ldots$ \\
\hline $\mathrm{D}$ & 17115 & A2156-3017 & 0.772 & 17179 & 0.000 & 0.772 & $\cdots$ \\
\hline $\mathrm{D}$ & 17710 & A2156-3017 & 0.772 & 17179 & 3.303 & 3.392 & $\cdots$ \\
\hline $\mathrm{D}$ & 17710 & A2156-3030A & 0.653 & 17156 & 3.631 & 3.690 & $\ldots$ \\
\hline $\mathrm{D}$ & 17710 & A2155-3033B & 0.441 & 17093 & 4.531 & 4.552 & $\cdots$ \\
\hline $\mathrm{D}$ & 18070 & A2156-2900 & 6.482 & 17739 & 0.536 & 6.504 & 16.6 \\
\hline $\mathrm{D}$ & 18070 & A2155-2851 & 7.319 & 18277 & 0.000 & 7.319 & 16.4 \\
\hline $\mathrm{D}$ & 18070 & A2156-3017 & 0.772 & 17179 & 8.454 & 8.489 & $\ldots$ \\
\hline \multicolumn{8}{|c|}{ Q $1230+0115$} \\
\hline $\mathrm{D}$ & 1487 & $\mathrm{~A} 1225+0152$ & 0.271 & 1298 & 0.000 & 0.271 & 16.3 \\
\hline $\mathrm{D}$ & 1487 & $12308+0147$ & 0.279 & 1643 & 0.000 & 0.279 & 15.3 \\
\hline $\mathrm{D}$ & 1487 & $\mathrm{~A} 1224+0146$ & 0.294 & 1275 & 0.000 & 0.294 & $\ldots$ \\
\hline $\mathrm{D}$ & 1663 & $12308+0147$ & 0.279 & 1643 & 0.000 & 0.279 & 15.3 \\
\hline $\mathrm{D}$ & 1663 & N4517A & 0.365 & 1530 & 0.000 & 0.365 & 12.9 \\
\hline $\mathrm{D}$ & 1663 & $\mathrm{~A} 1223+0117$ & 0.423 & 1473 & 0.000 & 0.423 & 17.1 \\
\hline $\mathrm{D}$ & 1742 & $12308+0147$ & 0.279 & 1643 & 0.000 & 0.279 & 15.3 \\
\hline
\end{tabular}


Table 10-Continued

\begin{tabular}{|c|c|c|c|c|c|c|c|}
\hline $\begin{array}{l}\operatorname{Abs}^{\mathrm{a}} \\
\text { Type } \\
(1)\end{array}$ & $\begin{array}{c}\text { Abs Vel } \\
\left(\mathrm{km} \mathrm{s}^{-1}\right) \\
(2)\end{array}$ & $\begin{array}{c}\text { Nearest } \\
\text { Galaxy } \\
(3)\end{array}$ & $\begin{array}{c}D_{\perp}^{\mathrm{b}} \\
\left(h_{70}^{-1} \mathrm{Mpc}\right) \\
(4)\end{array}$ & $\begin{array}{c}\text { Gal Vel } \\
\left(\mathrm{km} \mathrm{s}^{-1}\right) \\
(5)\end{array}$ & $\begin{array}{c}D_{c z}{ }^{\mathrm{c}} \\
\left(h_{70}^{-1} \mathrm{Mpc}\right) \\
(6)\end{array}$ & $\begin{array}{c}D_{\text {tot }}{ }^{\mathrm{d}} \\
\left(h_{70}^{-1} \mathrm{Mpc}\right) \\
(7)\end{array}$ & $\begin{array}{l}m_{B} \\
(8)\end{array}$ \\
\hline $\mathrm{D}$ & 1742 & N4517A & 0.365 & 1530 & 0.000 & 0.365 & 12.9 \\
\hline $\mathrm{D}$ & 1742 & $\mathrm{~A} 1223+0117$ & 0.423 & 1473 & 0.000 & 0.423 & 17.1 \\
\hline $\mathrm{D}$ & 1857 & $12308+0147$ & 0.279 & 1643 & 0.000 & 0.279 & 15.3 \\
\hline $\mathrm{D}$ & 1857 & N4517A & 0.365 & 1530 & 0.394 & 0.538 & 12.9 \\
\hline $\mathrm{D}$ & 1857 & $12277+0254$ & 0.562 & 1635 & 0.000 & 0.562 & 15.3 \\
\hline $\mathrm{P}$ & 2034 & $\mathrm{~A} 1228+0116$ & 0.162 & 2289 & 0.000 & 0.162 & 18.3 \\
\hline $\mathrm{P}$ & 2034 & $12270+0107$ & 0.293 & 2234 & 0.000 & 0.293 & 17.0 \\
\hline $\mathrm{P}$ & 2034 & N4536 & 0.585 & 1808 & 0.000 & 0.585 & 11.2 \\
\hline $\mathrm{D}$ & 2298 & $\mathrm{~A} 1228+0116$ & 0.162 & 2289 & 0.000 & 0.162 & 18.3 \\
\hline $\mathrm{D}$ & 2298 & $12270+0107$ & 0.293 & 2234 & 0.000 & 0.293 & 17.0 \\
\hline $\mathrm{D}$ & 2298 & $\mathrm{~A} 1227+0018$ & 0.749 & 2410 & 0.000 & 0.749 & $\ldots$ \\
\hline $\mathrm{P}$ & 5022 & $\mathrm{~A} 1235+0157$ & 2.375 & 4935 & 0.000 & 2.375 & $\ldots$ \\
\hline $\mathrm{P}$ & 5022 & $12360+0201$ & 2.467 & 5006 & 0.000 & 2.467 & 14.7 \\
\hline $\mathrm{P}$ & 5022 & $12361+0139$ & 2.511 & 5174 & 0.000 & 2.511 & 15.2 \\
\hline $\mathrm{P}$ & 6712 & $12268+0120$ & 0.726 & 6948 & 0.000 & 0.726 & 15.0 \\
\hline $\mathrm{P}$ & 6712 & N4493NW & 1.131 & 7012 & 0.000 & 1.131 & 14.9 \\
\hline $\mathrm{P}$ & 6712 & N4493SE & 1.134 & 6934 & 0.000 & 1.134 & 16.0 \\
\hline $\mathrm{P}$ & 7539 & $\mathrm{~A} 1228+0147$ & 0.522 & 7611 & 0.000 & 0.522 & 17.5 \\
\hline $\mathrm{P}$ & 7539 & $\mathrm{~A} 1228+0155$ & 0.733 & 7587 & 0.000 & 0.733 & 18.8 \\
\hline $\mathrm{P}$ & 7539 & $\mathrm{~A} 1228+0205$ & 1.069 & 7593 & 0.000 & 1.069 & 18.5 \\
\hline $\mathrm{D}$ & 9239 & $\mathrm{~A} 1227+0144$ & 0.523 & 9281 & 0.000 & 0.523 & 17.7 \\
\hline $\mathrm{D}$ & 9239 & $\mathrm{~A} 1228+0146 \mathrm{~A}$ & 0.581 & 9176 & 0.000 & 0.581 & 19.1 \\
\hline $\mathrm{D}$ & 9239 & $\mathrm{~A} 1226+0211$ & 1.859 & 8807 & 1.902 & 2.660 & 18.1 \\
\hline
\end{tabular}

aType of absorber, $\mathrm{D}=$ Definite $(S L \geq 4 \sigma)$ or $\mathrm{P}=$ Possible $(3 \sigma \leq S L<4 \sigma)$.

${ }^{\mathrm{b}}$ Galaxy distance, perpendicular to line-of-sight (LOS).

${ }^{\mathrm{c}}$ Absorber-galaxy distance along LOS, assuming retarded Hubble flow $\left( \pm 300 \mathrm{~km} \mathrm{~s}^{-1}\right)$.

dTotal 3D Euclidean absorber-galaxy distance, assuming retarded Hubble flow. 


\section{REFERENCES}

Baldwin, J. 1977, ApJ, 214, 679

Bahcall, J. N., Jannuzi, B. T., Schneider, B. P., Hartig, G. F., Bohlin, R., \& Junkkarinen, V. 1991a, in The First Year of HST Observations, ed. A. L. Kinney \& J. C. Blades (Baltimore: STScI), 46

Bahcall, J. N., Jannuzi, B. T., Schneider, B. P., Hartig, G. F., Bohlin, R., \& Junkkarinen, V. 1991b, ApJ, 377, L5

Bahcall, J. N., et al. 1993, ApJS, 87, 1

Bahcall, J. N., Bergeron, J., Boksenberg, A., Hartig, G. F., Jannuzi, B. T., Kirhakos, S., Sargent, W. L. W., Savage, B. D., Schneider, D. P., Turnshek, D. A., Weymann, R. J., \& Wolfe, A. M. 1996, ApJ, 457, 19

Bajtlik, S., Duncan, R. C., \& Ostriker, J. P. 1988, ApJ, 327, 570

Barlow, T. A., \& Tytler, D. 1998, AJ, 115, 1725

Brandt, J. C., et al. 1993, AJ, 105, 831

Brandt, J. C., et al. 1997, AJ, 114, 554

Burks, G. S., Bartko, F., Shull, J. M., Stocke, J. T., Sachs, E. R., Burbidge, E. M., Cohen, R. D., Junkkarinen, V. T., Harms, R. J., \& Massa, D. 1994, ApJ, 437, 630

Carswell, R. F., Whelan, J., Smith, M. G., Boksenberg, A., \& Tytler, D. 1982, MNRAS, 198, 91

Cen, R., Miralda-Escudé, J., Ostriker, J. P., \& Rauch, M. 1994, ApJ, 437, L9

Cowie, L. L., Songaila, A., Kim, T.-S., \& Hu, E. M. 1995 AJ, 109, 22.

Crenshaw, D. M., Kraemer, S. B., Boggess, A., Maran, S. P., Mushotzky, R. F., \& Wu, C.-C. 1999, ApJ, 516, 750

Cristiani et al. 1997, in Structure and Evolution of the IGM from QSO Absorption Lines, ed. P. Petitjean \& S. Charlot, (Paris: Editions Frontières), 165

Da Costa, L. N., et al. 1998, AJ, 116, 1

Davé, R., Hernquist, L., Katz, N., \& Weinberg, D. 1999, ApJ, 511,521

Fardal, M. A., Giroux, M. L., \& Shull, J. M. 1998, AJ, 115, 2206

Gibson, B. K., Giroux, M. L., Penton, S. V., Stocke, J. T., \& Shull, J. M. 2000a, AJ, submitted 
Gibson, B. K., Giroux, M. L., Penton, S. V., Putman, M. E., Stocke, J. T., \& Shull, J. M. 2000b, AJ, submitted

Gilliland, R. L., Morris, S. L., Weymann, R. J., Ebbets, D. C., \& Lindler, D. J. 1992, PASP, 104, 367

Gilliland, R., \& Hulbert, S. 1993, GHRS Instrument Science Report 55

Giovanelli, R., \& Haynes, M. P. 1984, AJ, 89, 758

Grogin, N. A., \& Geller, M. J. 1998, ApJ, 505, 506

Grogin, N. A., Geller, M. J., \& Huchra, J. P. 1998, ApJS, 119, 277

Haardt, F., \& Madau, P. 1996, ApJ, 461, 20

Hartmann, D., \& Burton, W. B. 1997, Atlas of Galactic Neutral Hydrogen (Cambridge: Cambridge Univ. Press)

Haynes, R. F., et al. 1999, in New Views of the Magellanic Clouds, ed. Y.-H. Chu, et al., (San Francisco: ASP), 63

Hernquist, L., Katz, N., Weinberg, D. H., \& Miralda-Escudé, J. 1996, ApJ, 457, L51

Huchra, J., Geller, M., Clemens, C., Tokarz, S., \& Michel, A. 1992, Bull. C.D.S., 41, 31

Huchra, J. P., Vogeley, M. S., \& Geller, M. J. 1999, ApJS, 121, 287

Impey, C. D., Petry, C. E., \& Flint, K. P. 1999, ApJ, 524, 536

Jannuzi, B. T., et al. 1998, ApJS, 118, 1

Lanzetta, K. M., Bowen, D. V., Tytler, D., \& Webb, J. K. 1995, ApJ, 442, 538

Kriss, G. A., et al. 2000, ApJ, in press

Lockman, F. J., \& Savage, B. D. 1995, ApJS, 97, 1

Lu, M., Savage, B., \& Sembach, K. 1994, ApJ, 417, L119

Lu, L., Sargent, W. L., Womble, D. S., \& Takada-Hidai, M. 1996, AJ, 472, 509

Mandel, J. 1984, The Statistical Analysis of Experimental Data, (New York, Dover).

Markwardt, C., 14 June 1999 Version, http://astrog.physics.wisc.edu/ craigm/idl/idl.html

Marzke, R. O., Huchra, J. P., \& Geller, M. J. 1996, AJ, 112, 1803

Miralda-Escudé, J., \& Ostriker, J. P. 1990, ApJ, 350, 1 
Moré, J., \& Wright, S. 1993, Frontiers in Applied Math, Number 14, Optimization Software Guide, SIAM.

Morras, R. 1983, AJ, 88, 62

Morris, S. L., Weymann, R. J., Savage, B. D., \& Gilliland, R. L. 1991, ApJ, 377, L21

Morris, S. L., Weymann, R. J., Dressler, A., McCarthy, P. J., Smith, B. A., Terrile, R. J., Giovanelli, R., \& Irwin, M. 1993, ApJ, 419, 524

Morris, S. L., \& van den Bergh, S. 1994, ApJ, 427, 696

Morton, D. C. 1991, ApJS, 77, 119

Murdoch, H. S., Hunstead, R. W., Pettini, M., \& Blades, J. C. 1986, ApJ, 309, 19

Murphy, E. M., Lockman, F. J., \& Savage, B. D. 1995, ApJ, 447, 642

Oegerle, W. R., Tripp, T. M., Sembach, K. R., Jenkins, E. B., Bowen, D. V., Savage, B. D., Shull, J. M., \& York, D. G. 2000, ApJ, in press

Penton, S. V., Shull, J. M., \& Stocke, J. T. 2000a, ApJ, submitted (Paper II)

Penton, S. V., Stocke, J. T., \& Shull, J. M. 2000b, in preparation (Paper III)

Penton, S. V., Shull, J. M., \& Stocke, J. T. 2000c, in preparation (Paper IV)

Penton, S. V., Shull, J. M., \& Edelson, R. 2000d, Database of IUE-AGN Ultraviolet Spectra, in preparation, see http://casa.colorado.edu/ iueagn

Rauch, M., Weymann, R. J., \& Morris, S. L. 1996 ApJ, 458, 518.

Russell, S. C., \& Dopita, M. A. 1992, ApJ, 384, 508

Sargent, W. L. W., Young, P. J., Boksenberg, A., \& Tytler, D. 1980, ApJS, 42, 41

Sargent, W. L. W. 1987 in QSO Absorption Lines, ed. J. C. Blades, D. Turnshek \& C. A. Norman (Cambridge: Cambridge Univ. Press), 1.

Savage, B., Sembach, K., \& Lu, L. 1995, ApJ, 449, 145

Savage, B., Sembach, K., \& Lu, L. 1997, AJ, 113, 2158

Savage, B., Tripp, M., \& Lu, L. 1998, AJ, 115, 436

Sherbert, L., \& Hulbert, S. 1997, GHRS Instrument Science Report 67 (Official Update)

Sembach, K. R., Savage, B. D., \& Lu, L. 1995a, ApJ, 449, 145

Sembach, K. R., Savage, B. D., Murphy, E., \& Lu, L. 1995b, ApJ, 451, 616 
Sembach, K. R., \& Savage, B. D. 1996, ApJ, 457, 211

Sembach, K. R., Savage, B. D., Lu, L., \& Murphy, E. M. 1999, ApJ, 515, 108

Shapiro, P. R., \& Benjamin, R. A. 1993, in Star-Forming Galaxies and their Interstellar Media, ed. J. Franco (Cambridge: Cambridge Univ. Press), 273

Shapiro, P. R., Giroux, M., \& Babul, A. 1994, ApJ, 427, 25

Shull, J. M., Stocke, J. T., \& Penton, S. V. 1996, AJ, 111, 72

Shull, J. M. 1997, in Structure and Evolution of the IGM from QSO Absorption Lines, ed. P. Petitjean \& S. Charlot, (Paris: Editions Frontières), 101

Shull, J. M., Penton, S. V., Stocke, J. T., Giroux, M. L., van Gorkom, J. H., Lee, Y. H., \& Carilli, C. 1998, AJ, 116, 2094

Shull, J. M., Penton, S. V., \& Stocke, J. T. 1999a, PASA, Vol. 16, No. 1, 95

Shull, J. M., Roberts, D., Giroux M. L., Penton, S. V., \& Fardal, M. A. 1999b, AJ, 118, 1450

Shull, J. M., Giroux, M. L., Penton, S. V., Tumlinson, J., Jenkins, E. B., Moos, H. W., Oegerle, W. R., Savage, B. D., Sembach, K. R., York, D. G., and the FUSE Science Team 2000, ApJ, submitted

Slavin, J. D., Shull, J. M., \& Begelman, M. C. 1993, ApJ, 407, 83

Songaila, A., Cowie, L. L., \& Hu, E. M. 1995

Steidel, C. C., 1995 in QSO Absorption Lines, ed. G. Meylan (Berlin: Springer), 139

Stocke, J. T., Shull, J. M., Penton, S. V., Donahue, M., \& Carilli, C. 1995, ApJ, 451, 24 (S95)

Tripp, T. M., Lu, L., \& Savage, B. D. 1998a, ApJ, 508, 200

Tripp, T.M., Lu, L., \& Savage, B. D. 1998b, in The Scientific Impact of the Goddard High Resolution Spectrograph, ASP Conference Series, Vol. 143, ed., J. C. Brandt, T. B. Ake, C. C. Peterson, 261

Tufte, S. L., Reynolds, R. J., \& Haffner, L. M. 1999, in Stromlo Workshop on High Velocity Clouds, ASP Conference Series, Vol 166, ed., B. K. Gibson, \& M. E. Putman, 231

Tytler, D., Fan, X.-M., Burles, S., Cottrell, L, Davis, C., Kirkman, D., \& Lin, Z. 1995 in QSO Absorption Lines, ed. G. Meylan (Berlin: Springer), 289

Van Gorkom, J. H., Bahcall, J. N., Jannuzi, B. T., \& Schneider, D. P. 1993, AJ, 106, 2213

Van Gorkom, J. C., Carilli, C. L., Stocke, J. T., Perlman, E. S., \& Shull, J. M. 1996, AJ, 112, 1397 
Wakker B. P., \& van Woerden, H. 1991, A\&A 250, 509

Wakker B. P., van Woerden, H., Schwarz U. J., Peletier, R. F., \& Douglas, N. 1996, A\&A, 306, L25

Weymann, R., Rauch, M., Williams, R., Morris, S., \& Heap, S. 1995, ApJ, 438, 650

Weymann, R., et al. 1998, ApJ, 506, 1

Wolfe, A. M. 1995, in QSO Absorption Lines, ed. G. Meylan (Berlin: Springer), 13.

York, D. G., Blades, J. C., Cowie, L., Morton, D. C., Songaila, A., \& Wu, C. 1982, ApJ, 225, 467

Zhang, Y., Meiksin, A., Anninos, P., \& Norman, M. 1997, ApJ, 495, 63 